\title{
COMPARISON OF CATALYTIC ETHYLENE POLYMERIZATION IN SLURRY AND GAS PHASE
}

Majid Daftaribesheli 


\section{Promotion Committee:}

Prof. dr. G. van der Steenhoven

Prof. dr. -Ing. habil. G. Weickert

Prof. dr. ir. W.P.M van Swaaij

Prof. dr. L. Böhm

Prof. dr. ir. V. Haddadi

Dr. ir. M. Van Sint Annaland

Dr. ir. G. Meier
University of Twente, chairman, The Netherlands

University of Twente, promoter, The Netherlands

University of Twente, The Netherlands

RWTH Aachen, Germany

Amir Kabir University, Tehran, Iran

University of Twente, The Netherlands

LyondellBasell, Frankfurt, Germany

The research described in this thesis was performed at the University of Twente-The Netherlands. The work described in this thesis is part of the Research programme of the Dutch Polymer Institute, PO Box 902, 5600 AX, Eindhoven, the Netherlands, projectnr. \#507.

Copyright (C) 2009 by Majid Daftaribesheli, Enschede, The Netherlands

No part of this work may be reproduced by any means, nor transmitted, nor translated into machine language without a written permission from the author.

ISBN 97-890-365-2838-2 


\title{
COMPARISON OF CATALYTIC ETHYLENE POLYMERIZATION IN SLURRY AND GAS PHASE
}

\section{PROEFSCHRIFT}

ter verkrijging van

de graad van doctor aan de Universiteit Twente,

op gezag van de rector magnificus, prof. dr. H. Brinksma,

volgens besluit van het College voor Promoties

in het openbaar te verdedigen

op donderdag 11 juni 2009 om 16:45 uur

\author{
door \\ Majid Daftaribesheli \\ geboren op 16 augustus 1966 \\ te Ghaemshahr, Iran
}


Dit proefschrift is goedgekeurd door de promotor

Prof. dr. -Ing. habil. Günter Weickert 
To my parents, wife, and sons 



\section{Table of contents}

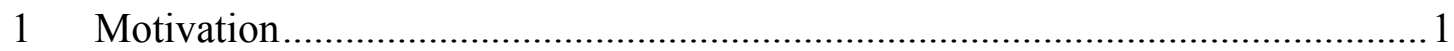

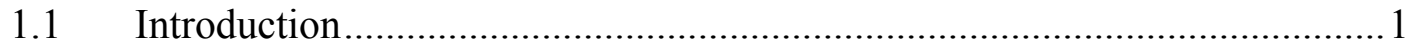

1.1.1 Hostalen: A Typical Slurry Process ......................................................

1.1.2 Innovene G: A Typical Gas-phase Process........................................... 4

1.2 Comparison of Slurry and Gas-phase Polymerization...................................5

1.2.1 Industrial Point of View......................................................................

1.2.2 The Scientific Prospective on Slurry and Gas Phase ..............................6

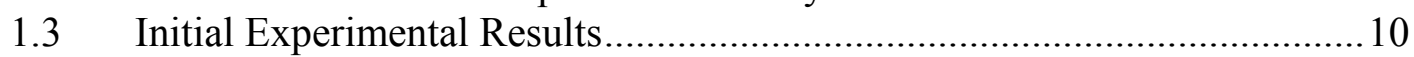

1.3.1 The Influence of $\mathrm{n}$-Hexane ................................................................ 10

1.3.2 The Influence of Hydrogen ..............................................................11

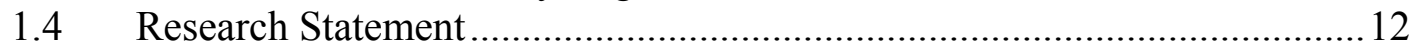

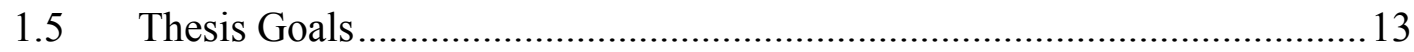

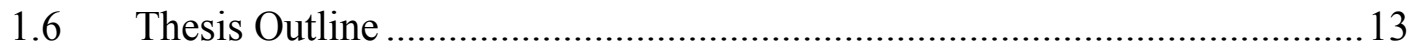

2 Experimental and Theoretical Methods .......................................................... 17

2.1 Experimental Procedure ....................................................................... 17

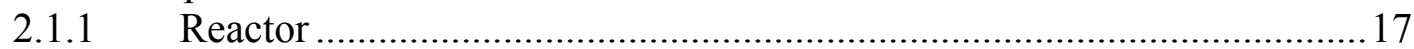

2.1.2 Catalyst Handling and Preparation .................................................... 18

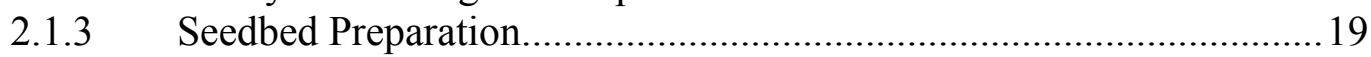

2.1.4 Gas-Liquid Purification ................................................................... 19

2.1.5 Polymerization Procedures ...............................................................21

2.2 Estimation of Reaction Rate ………………………….............................21

2.3 Particle Size Distribution Measurements and Analyses ..............................22

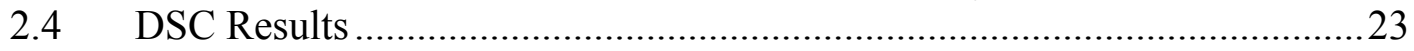

$2.5 \quad$ Molecular Weight Distribution ...............................................................24

$2.6 \quad$ Scanning electron microscopy (SEM) …………….................................24

2.7 Transmission Electron Microscopy (TEM) …………………………….....2.

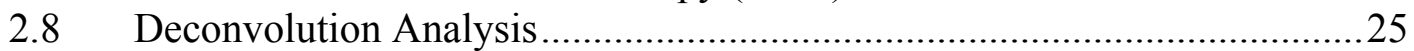

2.9 Prediction of Ethylene and Hydrogen Concentration in Slurry and Gas-

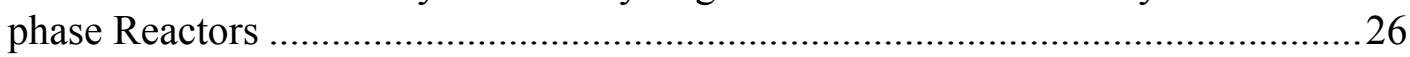

2.9.1 The Sanchez-Lacombe Equation of State Model................................27

2.9.2 The Soave-Redlich-Kwong Cubic Equation of State ...........................2 28

2.9.3 Initial Component Concentration Prediction ......................................29

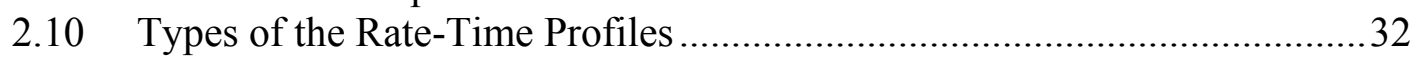

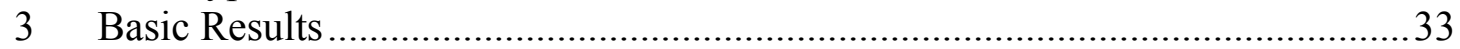

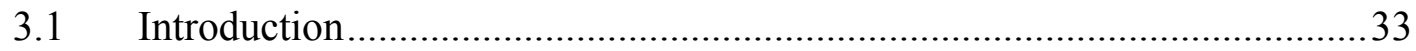

3.2 Reproducibility of Experiments ……………………....................................

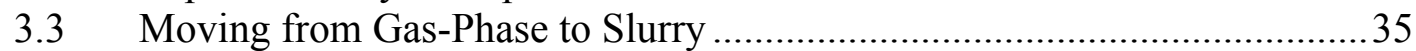

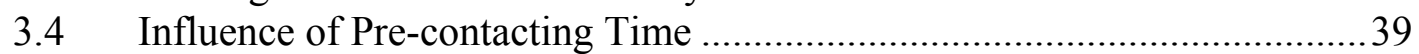

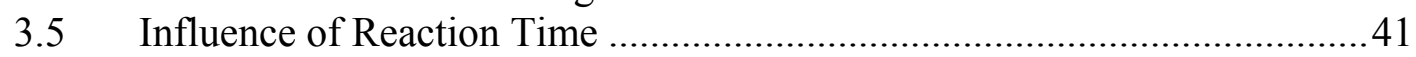

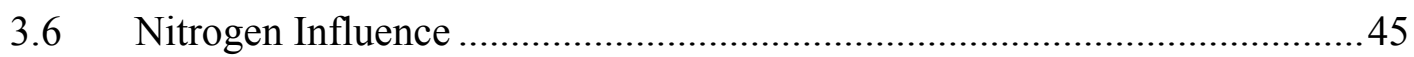

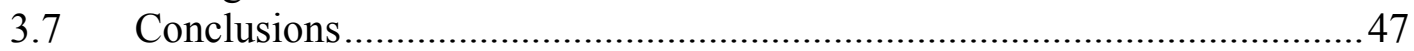

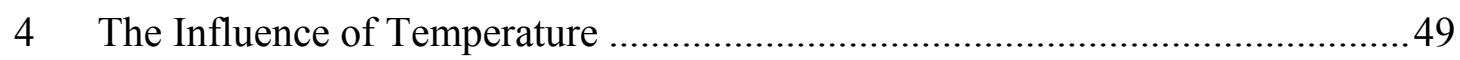

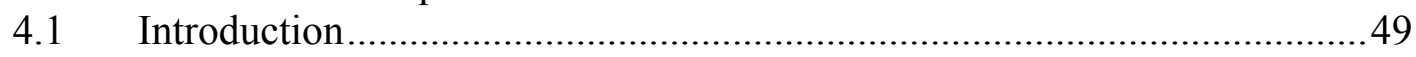

4.2 Slurry polymerization in the absence of hydrogen ......................................51

4.2.1 Polymerization Rate Profiles .............................................................51

4.2.2 Molecular weight and crystallinity .....................................................55

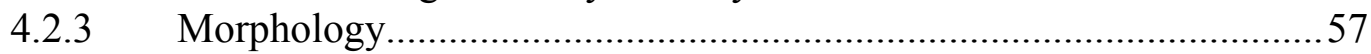


4.3 Slurry Polymerization in the Presence of Hydrogen...............................60

4.3.1 Polymerization Rate Profiles ..............................................................6 60

4.3.2 Molecular Weight and Crystallinity................................................62

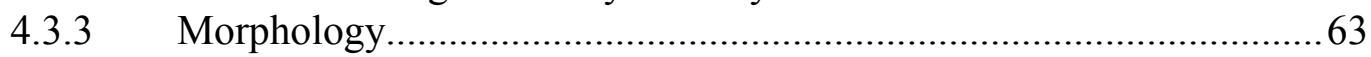

4.4 Gas-Phase Polymerization in the Presence of Hydrogen...........................67

4.4.1 Polymerization Rate Profiles ............................................................6 68

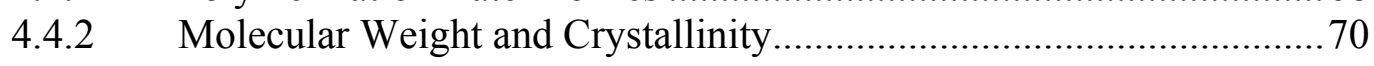

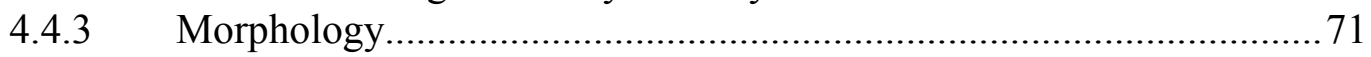

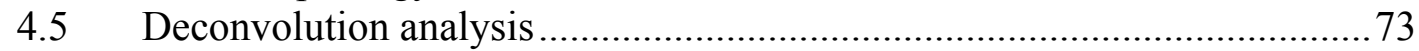

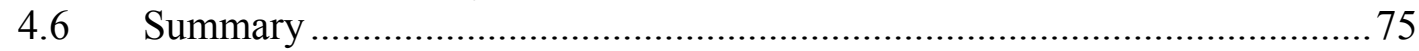

4.7 Conclusions for Process Modeling ........................................................ 76

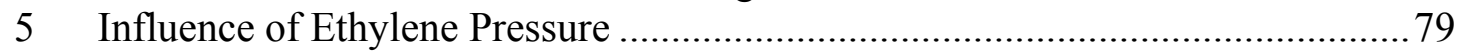

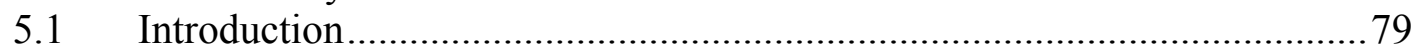

5.2 Slurry polymerization in absence of hydrogen ..................................... 80

5.3 Gas phase polymerization at constant hydrogen pressure ......................... 85

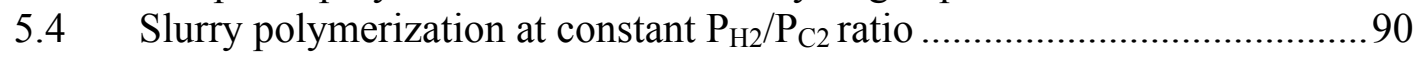

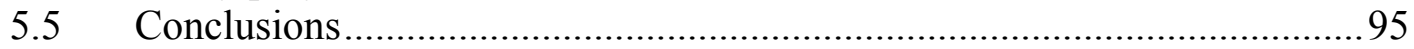

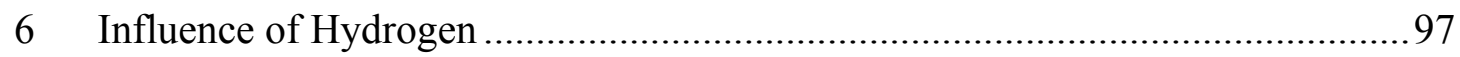

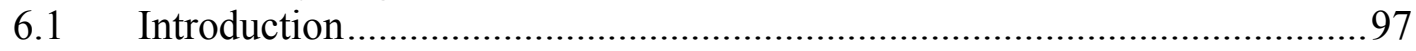

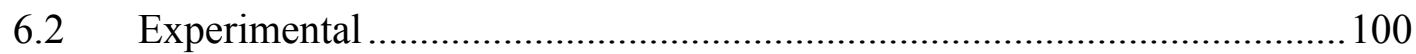

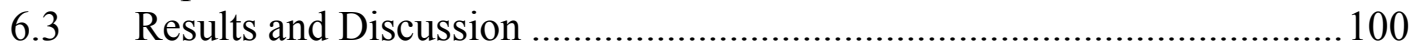

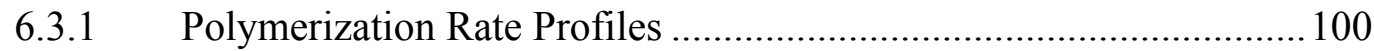

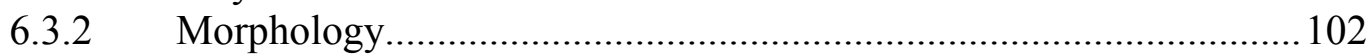

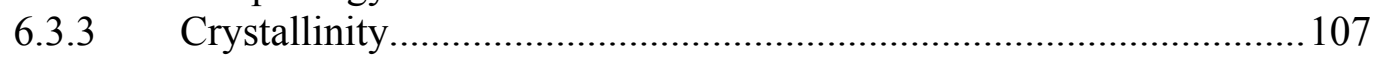

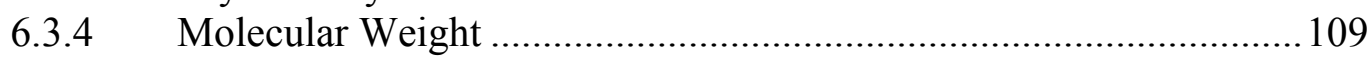

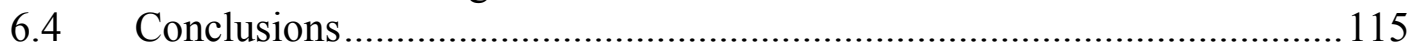

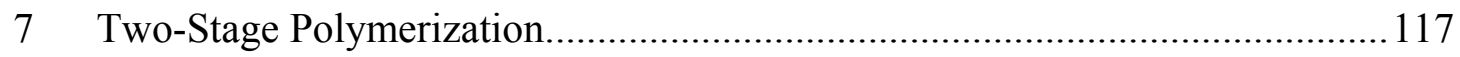

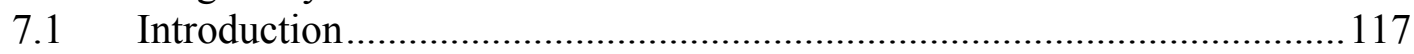

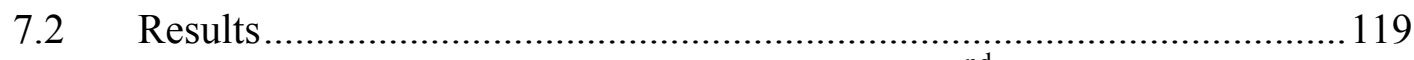

7.2.1 Slurry Polymerization: Hydrogen Feed in the $2^{\text {nd }}$ Step .........................119

7.2.2 Slurry Polymerization: Changing the Ethylene and the Hydrogen

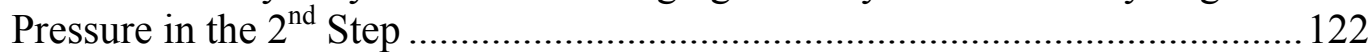

7.2.3 Gas-Phase Polymerization: Changing the Ethylene and Hydrogen

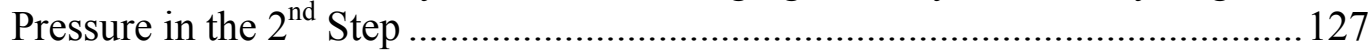

7.2.4 Slurry and Gas-Phase Polymerization: Changing the Ethylene Pressure

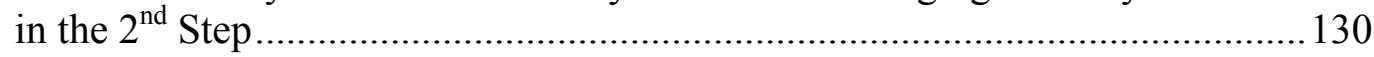

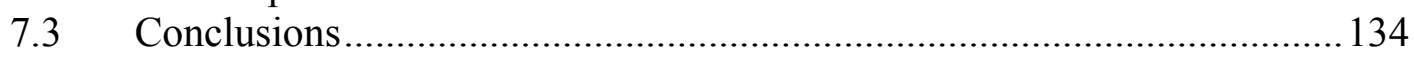

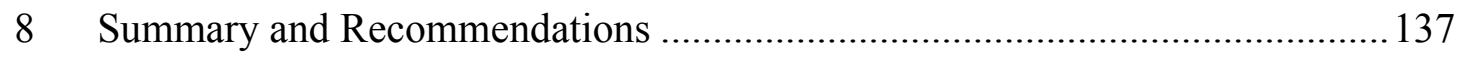

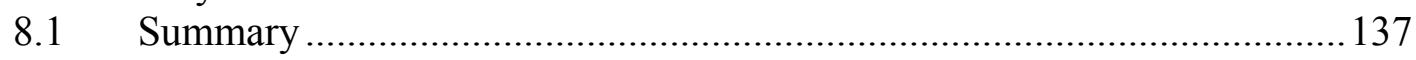

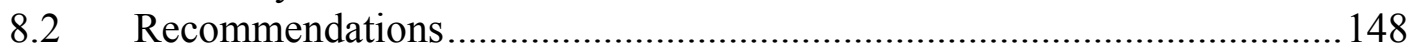

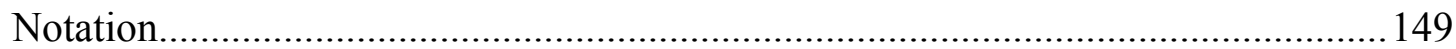

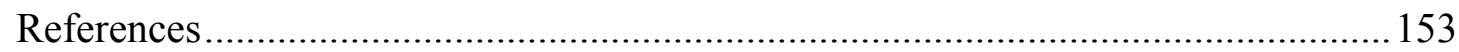

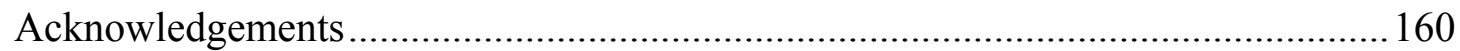

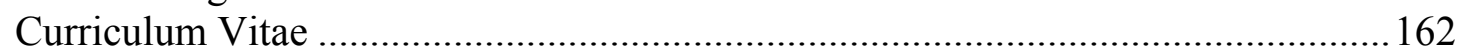




\section{Chapter 1}

\section{Motivation}

\subsection{Introduction}

Polyethylenes accounted for $30 \%$ of the total annual world polymer consumption in 2007 (more than 70 million tonnes in 2007) [1] and are the most widely utilized synthetic polymers. There are many scientific, industrial and commercial reasons for this enormous consumption, such as good chemical resistance, zero toxicity, bio-acceptability, good physical and mechanical properties, low cost, ease of fabrication, good raw material availability and low environmental impact [2-6].

On the basis of the product's properties, polyethylene can be commercially classified into five major types with different densities and branching which lead to different properties. These types include:

- very low-density polyethylene (VLDPE)

- low-density polyethylene (LDPE)

- linear low-density polyethylene (LLDPE)

- high-density polyethylene (HDPE)

- ultra high molecular weight polyethylene (UHMWPE).

The properties of these polymers, with the exception of LDPE, are varied mainly by changing the hydrogen concentration as a termination agent for controlling the molecular weight, and by co-monomer type and its concentration along the molecular backbone for controlling the crystallinity and density of polymer. The influence of hydrogen on the particle morphogenesis is one of the major topics of this work.

This thesis will deal with $\mathrm{HDPE}^{1}$, the PE type with the highest worldwide consumption. HDPE with a density of greater or equal to $0.94 \mathrm{~kg} / \mathrm{m}^{3}$ is usually termed linear polyethylene due to its low short branching content or lack of branching, which leads to high crystallinity polyethylene than other polyethylenes. HDPE can be made using any catalytic multi-site (e.g. Chromium catalysts, Ziegler-Natta catalysts) or using single-site catalysts (e.g. metallocenes) by homo- or copolymerization of ethylene; in the latter case using a very low content of co-monomers such as 1-butene or 1-hexane. Medium-density polyethylenes in a density range of 0.926 to $0.940 \mathrm{~kg} / \mathrm{m}^{3}$ can be classified as HDPE due to significant similarities with this class of polymer. Although other applications do exist, HDPE is

\footnotetext{
${ }^{1}$ Without any type of co-monomer
} 
predominantly used in products and packaging such as bottles, tubes, containers, water pipes, gas pipes and toys.

On the basis of the reactor conditions (e.g. pressure, temperature) and the flow regime (e.g. slurry, solution and gas-phase), HDPE can be produced industrially by three different processes which can be commercially divided into three types: (1) solution process; (2) slurry process; and (3) gas-phase process. The various polymerization processes and reactor operating conditions are listed in Table 1.1 [7].

Table 1.1-HDPE process and reactor operating conditions. Adopted from [7]

\begin{tabular}{|c|c|c|c|}
\hline & Solution & Slurry & Gas-phase \\
\hline Reactor type & CSTR & Loop or CSTR & Fluidized or stirred bed \\
\hline Pressure, atm & $\sim 100$ & $30-35$ & $30-35$ \\
\hline Temperature, ${ }^{\circ} \mathrm{C}$ & $140-200$ & $85-110$ & $80-105$ \\
\hline Loci of polymerization & Solvent & Solid & Solid \\
\hline Density, $\mathrm{g} / \mathrm{cm}^{3}$ & $0.910-0.970$ & $0.930-0.970$ & $0.910-0.970$ \\
\hline MFI, g/10 min & $0.5-105$ & $<0.01-80$ & $<0.01-200$ \\
\hline
\end{tabular}

Usually, combinations of reactor are used, for example one or two loop reactors are combined with one or two fluidized bed reactors. A special case is the BORSTAR process that runs the loop reactor under supercritical conditions with propane as the slurrifying agent [8].

This work will focus on a comparison between the slurry and gas phase processes. Why do different products result even if the same catalyst is used?

From the $\mathrm{S}$ curve of polyethylene technology $[9,10]$, it can be seen that particularly slurry and gas-phase polyethylene processes caused revolutionary improvements in polyethylene technology; see Figure 1.1.

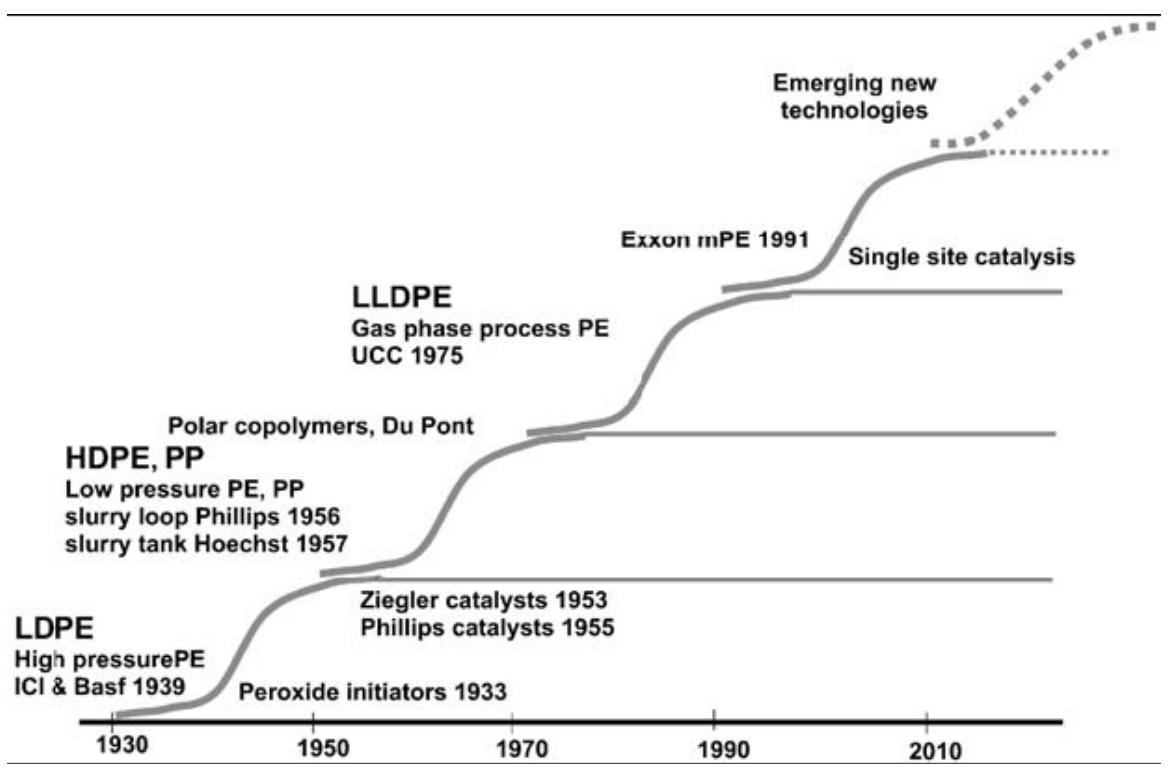

Figure 1.1-S curve of polyethylene technology taken from $[9,10]$ 
Slurry, gas-phase and hybrids processes still remain competitive because of their different properties that meet the product demand profiles of the market. Some of the most important licensors which produce HDPE are listed in Table 1.2.

Table 1.2-Gas-Phase and Hybrids Processes for ethylene polymerization

\begin{tabular}{|c|c|c|c|}
\hline \multicolumn{2}{|c|}{ Slurry low pressure } & \multicolumn{2}{|c|}{ Gas-phase } \\
\hline Brand Name & Licensor & Brand Name & Licensor \\
\hline Hostalen BM & LyondellBasell & Innovene $\mathrm{G}$ & Ineos \\
\hline Hostalen ACP & LyondellBasell & Spherilene C & LyondellBasell \\
\hline Mitsui CX & Mitsui & Spherilene S & LyondellBasell \\
\hline \multicolumn{2}{|c|}{ Slurry loop process } & Evolue & Mitsui \\
\hline Brand Name & Licensor & Univation & Exxon and Dow \\
\hline Philips & ChevronPhilips & \multicolumn{2}{|c|}{ Hybrids of slurry and gas-phase } \\
\hline Ineos $\mathrm{S}$ & Ineos & Borstar PE & Borealis \\
\hline
\end{tabular}

Two typical industrial processes (Hostalen and Innovene G) will shortly be analyzed for a basic introduction to the major topic of this work. The reader can find descriptions of more processes in the literature[7, 11, 12].

\subsubsection{Hostalen: A Typical Slurry Process}

Figure 1.2 shows a simplified process schematic of the Hostalen process [13].

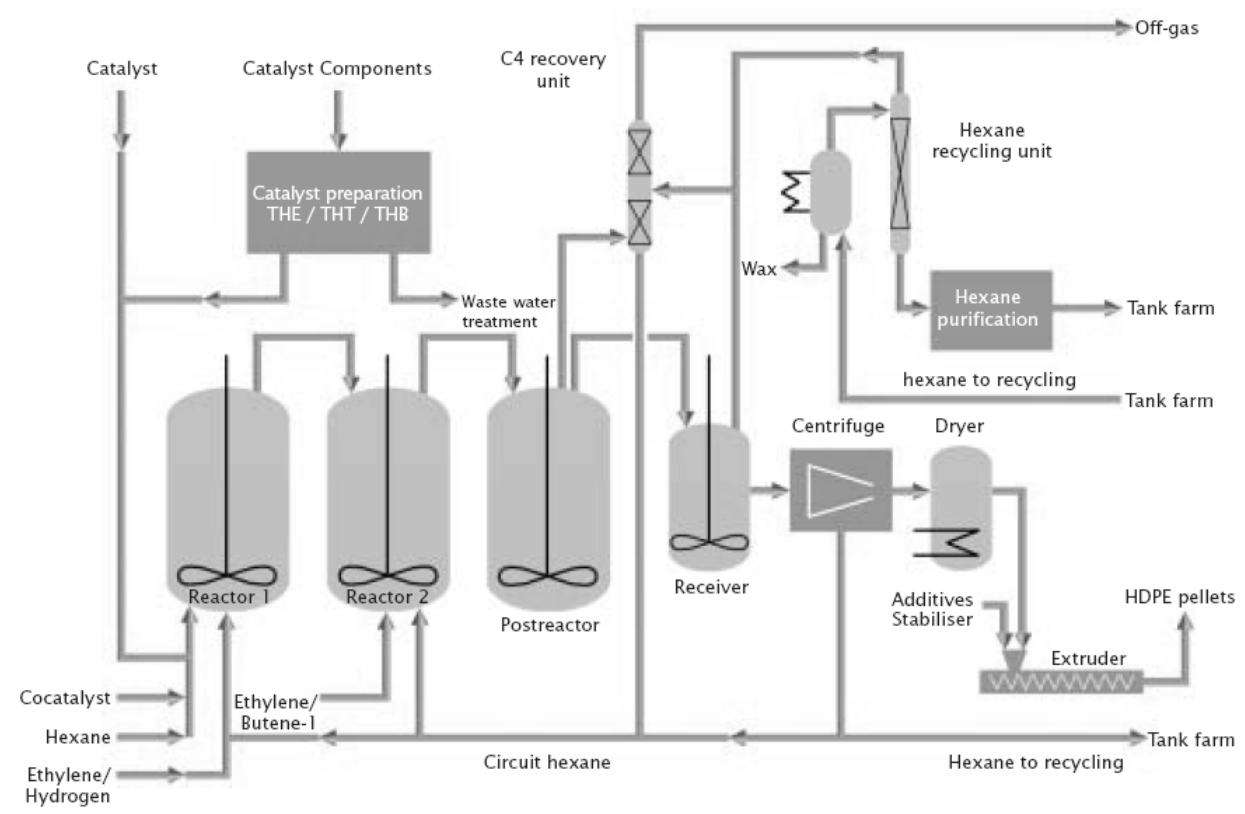

Figure 1.2- Slurry ethylene polymerization (Hostalen processes) [13] 
The Hostalen process consists of two continuous stirred-tank reactors (CSTR) that can be operated in parallel or in series, depending on which grade of polymer is required. The process is designed to produce either unimodal (broad or narrow molecular weight distribution polymer) or bimodal polymer by using Ziegler-Natta catalysts. For bimodal polymer production, a high concentration of hydrogen in the first reactor and a low concentration of hydrogen plus a small amount of co-monomer in the second reactor is used. The ethylene concentration in the second reactor is much higher than the first reactor.

\subsubsection{Innovene G: A Typical Gas-phase Process}

Figure 1.3 shows the schematic of the Ineos "Innovene G" plant. In this process, the catalyst and co-catalyst are fed to a slurry stirred-tank reactor in which pre-polymerization occurs. Pre-polymerization under mild conditions helps to prevent hot spots or the production of fines which is caused by high heat generation and growth stress inside the particles.

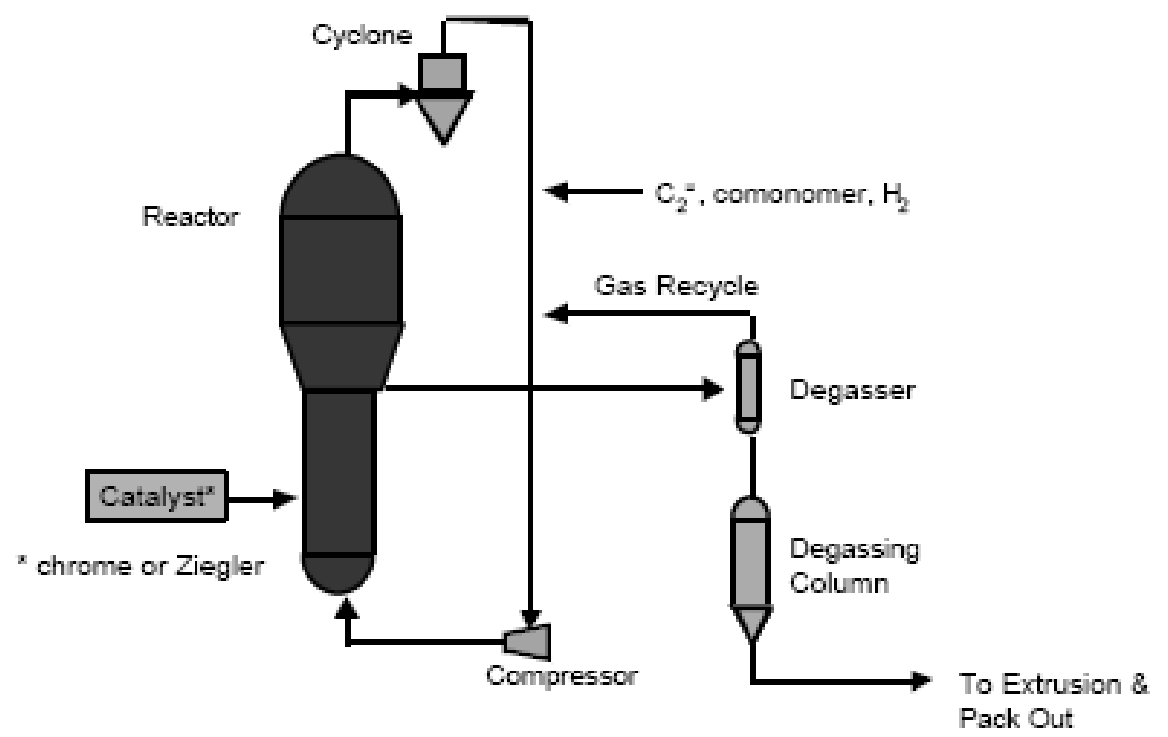

Figure 1.3-Gas-phase ethylene polymerization plant (Innovene G)

The pre-polymer is transferred to a dryer where hot nitrogen evaporates the solvent. Then the pre-polymer powder, as a catalyst for the main polymerization reactor, is fed continuously to the fluidized bed reactor. As can be seen in Figure 1.3, the fluidization reactor comprises two main parts: a cylindrical part and a disengagement part [14]. The cylindrical part is equipped with a gas distributor in order to fluidize the content of the bed. The disengagement part reduces the velocity of flowing gas and consequently disengages the polymer particle from the outgoing gas. Cyclones are installed to remove fines coming from circulated gas from the top of the reactor. Fines from bottom of the cyclones, which are usually quite active, are returned to the reactor for further polymerization. Circulating gases 
from top of the cyclones are passed through a heat exchanger(s) and then mixed with a certain quantity of fresh feeds to fix the composition of components in the reactor. Finally, the gases are compressed and returned to the bottom of the reactor. Ethylene as a monomer, butene-1 or hexene-1 as co-monomer, hydrogen as a chain transfer and nitrogen as an inert gas are introduced at different points of the circulating-gas pipe in order to achieve perfect mixing and to prevent condensation which could damage the blades of the compressor or block the holes of the gas distributor. The circulating gas fluidizes the bed and removes the heat of reaction.

The so-called "Condensed mode" was introduced by Jenkins et al. [15] and involves a liquid hydrocarbon being injected into the bed to remove reaction heat by evaporation. A cooling system in the loop condenses and separates the liquid from the circulating gas, and the gas is then injected back into the bed.

\subsection{Comparison of Slurry and Gas-phase Polymerization}

\subsubsection{Industrial Point of View}

Due to the use of solvent in slurry processes, some additional equipment is required, for instance: solvent stores, solvent purification, solvent removal and powder drying sections. Therefore, gas-phase processes are more compact and simpler, the costs of gas-phase plants are lower $[16,17]$ and their environmental impact is less when compared to slurry processes [6].

In gas-phase processes, no mass transfer limitation gas-liquid can occur, and therefore, there is no polyethylene, monomer, co-monomer and hydrogen solubility concern in the solvent medium during the polymerization [18]. Therefore, more soluble PE, for example most medium density (MDPE) and linear low density polyethylene (LLDPE), are produced in gas-phase processes. These advantages allow the operation of gas phase plants as multipurpose plants for producing a wide range of polyethylenes with different densities and melt flow indexes (MFI). In addition, gas-phase processes create no wall sheeting or fouling due to the polyethylene's solubility in the solvent medium which is the main problem in slurry processes. However, wall sheeting is also a major problem in gas phase reactors, as described below.

Still, there remains a significant demand for slurry processes in the market, especially because of its advantages:

- mild operating conditions

- high monomer conversion

- ease of heat removal

- relative ease of processing.

In addition, due to some problems that can occur in gas phase processes: 
- Production of more off-spec polymer during grade change especially for transition from HDPE to LLDPE or changing from one catalyst to another catalyst type.

- Agglomeration and lump formation due to poor heat removal from growing polymer particles, leading to the formation of hotspots followed by sintering of the polymer.

- Disintegration of the growing polymer particle due to undesirable stress (physical, mechanical or chemical) leading to the formation of fines (smaller than 125 micron $[19,20])$. Increasing the fines content in the gas-phase reactor is catastrophic for all gas-phase plants, leading to a loss of homogeneity of fluidization and eventually leading to the blockage of subsequent process units.

- Electrostatic charge leading to agglomerate formation or wall sheeting, especially near the inclined part of the disengagement zone of gas phase reactor.

Finally, most research is carried out into slurry due to its ease of operation and temperature control, and most catalysts have been developed in a series of slurry experiments. However, because of the differences between gas and slurry processes, the transfer of results from slurry to the gas-phase is not easy [21,22] even when the same catalyst is used. Clearly, an extensive investigation into the process fundamentals is required, and this is one of the motivating arguments for this work. The following discussion comes closer to achieving final definition of the target.

\subsubsection{The Scientific Prospective on Slurry and Gas Phase}

Simplifying ${ }^{1}$ in this work, we draw a distinction between the following phases in a slurry HDPE process:

- gas phase (containing ethylene, hydrogen, inert gas, vaporized liquid)

- liquid phase (solution of gases and co-catalyst, solid polymer is dispersed)

- particle phase, consisting of the following phases

o catalyst $\left(\mathrm{MgCl}_{2}\right.$-supported $\mathrm{TiCl}_{3}$ with coordinated co-catalyst)

o pores filled with solvent and all soluble components

o crystalline PE (does not contain gases and liquids[23])

o amorphous PE (swollen with solvent, gases, co-catalyst).

The growing particles are suspended in the "inert" solvent ${ }^{2}$ (usually C5-C 8 alkanes). Such a slurry polymerization process can be controlled by mass transfer limitations between these three phases:

- gas-solvent

- solvent-particle

- intra-particle (within the pores and within the amorphous polymer)

\footnotetext{
${ }^{1}$ We neglect: polymer in solution (amorphous low-molecular-weight PE),

${ }^{2}$ We will show that this slurrifying agent is not at all "inert" from the chemical engineer's point of view
} 
Heat transfer limitations, intra-particle or particle-solvent, are hardly possible in slurry. The solvent serves as a good heat transfer medium, thereby helping to avoid overheating phenomena.

For a typical gas-phase HDPE process, we distinguish the following two phases:

- gas phase (ethylene, hydrogen and inert gas)

- particle phase, consisting of four sub-phases

o catalyst $\left(\mathrm{MgCl}_{2}\right.$-supported $\mathrm{TiCl}_{3}$ with coordinated co-catalyst)

o pores filled with gas

o crystalline PE

o amorphous PE (swollen with gases, containing co-catalyst[23])

A liquid phase is only present in case of "condensed mode" operation - which is outside the scope of this work.

In both processes - gas and slurry - the polymerization process starts within a porous solid phase that does not contain any polymer, but consists of $\mathrm{MgCl}_{2}$ and $\mathrm{TiCl}_{3}$, often precontacted with the co-catalyst (often TEA or TIBA). $\mathrm{MgCl}_{2}$ fragments under the influence of the polymer production ${ }^{1}$ of more than 1 billion active sites per catalyst particle. Magnesium chloride with typically $10 \% \mathrm{TiCl}_{3}$ initially forms the continuous phase, but is distributed within the polymer after reaching yields higher than $1 \mathrm{~g}$ polymer / $\mathrm{g}$ catalyst, which can be the case $<1$ second under "industrial" conditions. For the characterization of the single particle behaviour, see [24-28].

From the above brief analysis of the thermodynamic phases, it become clear that the performance of the process (kinetics) and the product properties depend strongly on microscale processes such as sorption, diffusion, swelling, and particle morphology development around the active sites. These micro-processes are different for slurry- and gas phase ethylene polymerization. The polymerization rate depends on monomer and hydrogen concentration close to the active site of the catalyst, and these can be completely different for gas and slurry phases, also due to the different solubility of the monomers in solvent and PE [24].

Two others vital limitations need to be taken into account.

1. Heat and mass transfer at mesoscales (interparticle and intraparticle)

2. Limited catalyst productivity due to the thermal deactivation of active sites as a consequence of the dramatic initial temperature rise of the growing polyethylene particle especially in the gas phase. The adiabatic temperature rise of the ethylene polymerization is about $1800 \mathrm{~K}$.

Additionally, the particle size distributions of catalysts used in the slurry and gas phase processes are very different. The most heterogeneous Ziegler-Natta catalyst systems used in olefin polymerization have a mean particle diameter of 5-100 $\mu \mathrm{m}[29,30]$. In gasphase processes, the size of catalyst particles, including pre-polymers, is greater (50-300 micron) than those used in slurry processes (5-20 micron). Heat and mass transfer properties of the growing particles are functions of the particle size [31, 32], even if the catalyst

\footnotetext{
${ }^{1}$ This holds true for PE and PP - however, the crystallization rate of PE is faster, which is important for interpreting the "in-situ" formed morphology
} 
preparation guaranteed a homogeneous catalyst concentration within the carrier (which is probably seldom the case).

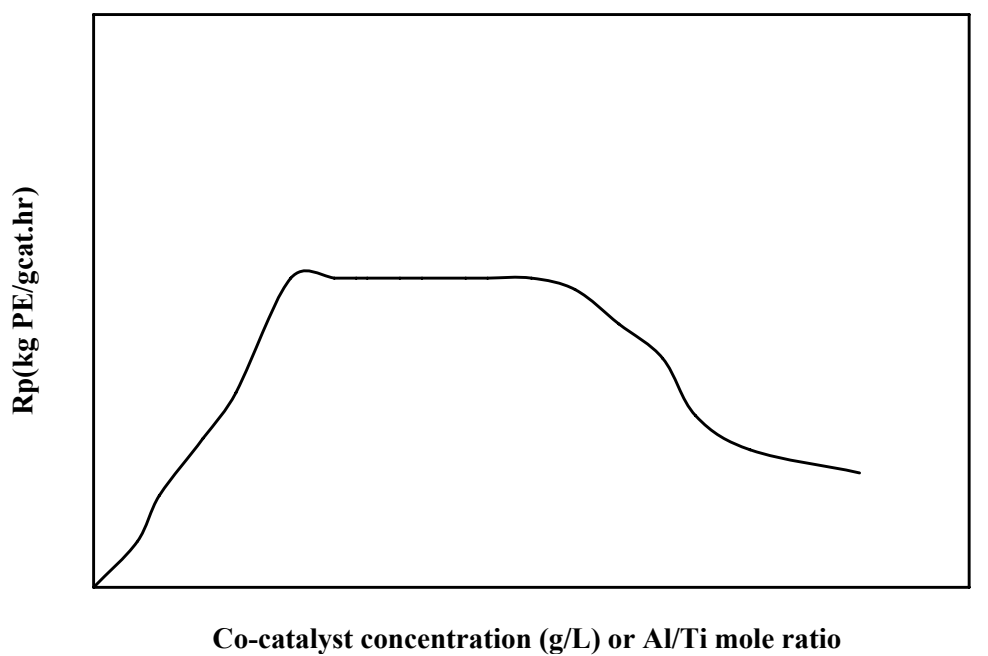

Figure 1.4-Effect of co-catalyst concentration or $\mathrm{Al} / \mathrm{Ti}$ mole ratio on polymerization rate

Furthermore, high activity ZN catalyst systems require co-catalysts to promote high polymerization activity. The co-catalyst concentration around the active sites influences the polymerization kinetic profile during the whole process: activation, maximum polymerization rate and catalyst decay are all strongly influenced. The diffusivity of a relatively big molecule such as TEA or TIBA differs significantly when comparing gas and slurry polymerization. The influence of the type and concentration of co-catalysts, and the Al/Ti molar ratio on the activity and productivity of $\mathrm{ZN}$ catalysts and the property of produced polyethylene have been the subjects of intensive research in both slurry and gas-phase ethylene polymerization [33, 34]. For instance, Figure 1.4 which has been reported by many researchers in various ways shows that by increasing co-catalyst concentration or $\mathrm{Al} / \mathrm{Ti}$ molar ratio to a certain value, the reaction rate increases and reaches a plateau. Further increase in the co-catalyst concentration leads to a decrease in the reaction rate [35-38]. Due to different sorption, diffusion and back diffusion of co-catalyst during the course of growth of catalyst/polymer particle in slurry and gas-phase polymerization, the co-catalyst concentration near the active site decreases ( the dilution effect), which may lead to a decrease in the reaction rate as shown in Figure 1.4. Based on this interpretation, the decreasing rate must differ between the slurry and gas phase. Unfortunately, the influence of co-catalyst mass transfer has not yet been sufficiently studied.

Generally, for all participating components the solubility equilibrium is disturbed by the monomer consumption and polymer production at the active sites. MONOMER flows from the particle surface through the pores and through the amorphous polymer to the active sites which are more or less embedded in the polymer produced. POLYMER flows countercurrently from active sites to the particle surface under extreme flow conditions, and especially the viscosity in the polymer phase ("micro-viscosity") is much higher in the gas phase than in slurry. Conversely, the viscosity in the pores is higher for a slurry process. The 
polymer production leads to a permanent dilution effect for all components near the active sites.

The thermodynamic scheme is demonstrated in Figure 1.5: the gas, liquid and solid phases are permanently exchanging components while the volume of the solid phase grows.

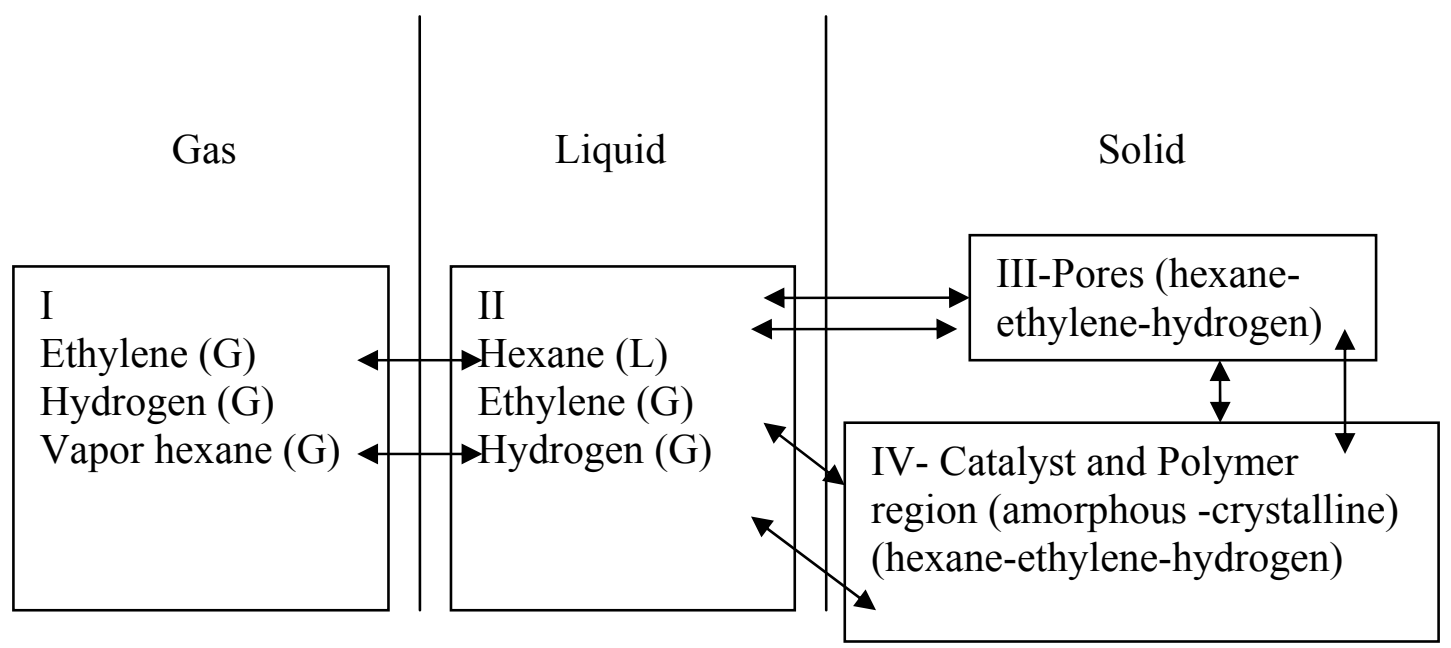

Figure 1.5-Phase participation in slurry polymerization

Figure 1.6 is a scheme of the polyethylene structure and shows the most likely locations of active centres in the crystalline-amorphous structure of polyethylene. Due to the high compactness of crystalline regions, monomers can hardly reach the active centres which are covered by too much crystalline polymer[23, 30].
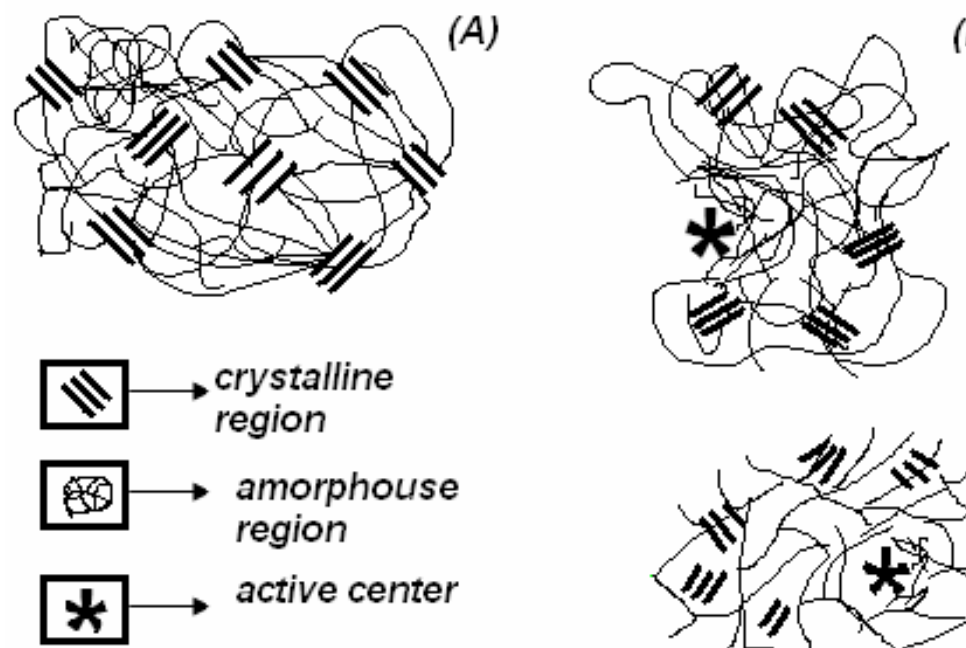

(B)

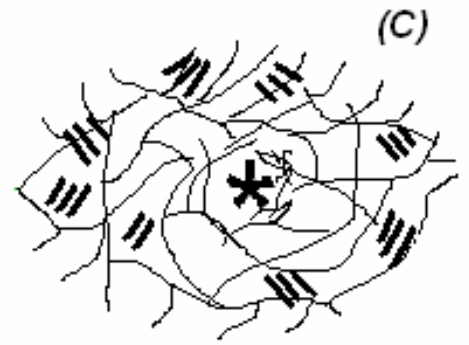

Figure 1.6-Polyethylene structure and active centre location A. Polyethylene structure B. Active centres on the polymer surface or inside the pores $\mathrm{C}$. Active centres in the amorphous phase 
Particle fragments under the influence of the growth stress. This generates both new active sites at the surface and new pores, and the polymerization rate increases initially. Depending on how fast the reaction proceeds, either within the amorphous region or on the surface of polymer, the transport rates between the gas, liquid and solid phases change.

The morphology of growing catalyst/polymer particles changes according to the above described processes, and is determined by both the brittleness of the $\mathrm{MgCl} 2$ carrier and the growth stress generated by the polymer produced - taking into account the rate of crystallization and encapsulation processes around the active sites. Therefore, the morphology and polymerization rate influence each other, but this interaction depends on several chemical and physical factors, the role of which is not yet completely clear, because, the conditions near the active sites cannot be measured directly.

Can modelling help?

Considerable effort has gone into the modelling of olefin polymerizations; see, for example, "solid core model" [39], "polymeric core model" [39] "multigrain model" [40-42], "dusty-gas model", "multi-site model" [43, 44]. However, due to the existence of a huge number of variables and the complexity of the process at the micro, meso and macro level, a widely accepted generalizing model that can describe the morphogenesis as function of growth stress and polymer quality has not yet been developed, whether for slurry or gas phase polymerizations.

Comparing some single aspects of slurry and gas-phase ethylene polymerization has been the focus of intensive research in the academic and industrial spheres, for example, [28, $33,34,45,46]$.

However, all this work still did not lead to a generally accepted model that can explain HOW the polymerization rate and morphology interact under varying reaction conditions, especially when various concentrations of hydrogen are used to control the molecular weight. Not could it explain WHY this interaction is so different in gas and slurry? Some initial experiments have been done to specify this problem and to select a set of experimental methods for clarifying the objectives of this work - see Chapters 3 to 7 for descriptions of the complete set of experimental parameters.

\subsection{Initial Experimental Results}

\subsubsection{The Influence of n-Hexane}

Figure 1.7 shows the results of three experiments carried out under constant operating conditions and using the same catalyst preparation method as that used for the "gas phase catalyst, Cg". The polymerization rate profile for the run GGE (pure gas-phase by using salt as bed material), starts quickly, reached the peak within 8 minutes, and decreased rapidly. By increasing the amount of n-hexane (120ml in GSE1 and 700ml in GSE2), the shape of the profile changes significantly. 


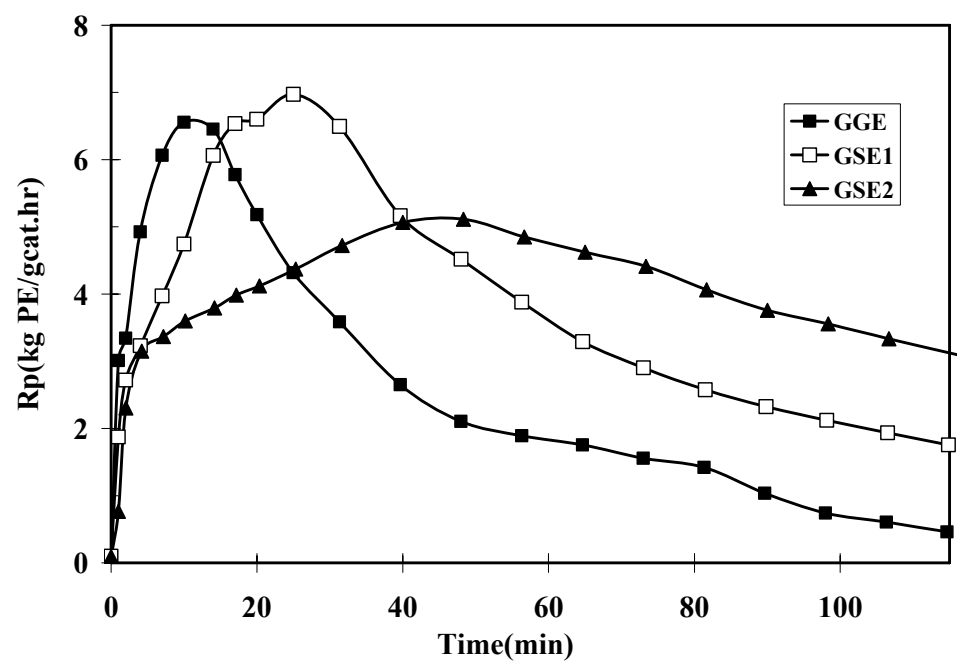

Figure 1.7-The influence of the amount of solvent on homo-ethylene polymerization at $\mathrm{T}=80^{\circ} \mathrm{C}$, $\mathbf{P}_{\mathrm{C} 2}=\mathbf{2}$ bar and $\mathbf{P}_{\mathrm{H} 2}=\mathbf{2}$ bar

Figure 1.8 shows the corresponding particle size distribution of the three previously mentioned experiments. Despite changing the shape of reaction rate which leads to different yield, no remarkable changes can be seen in the profile of particle size distribution normalized with yield.

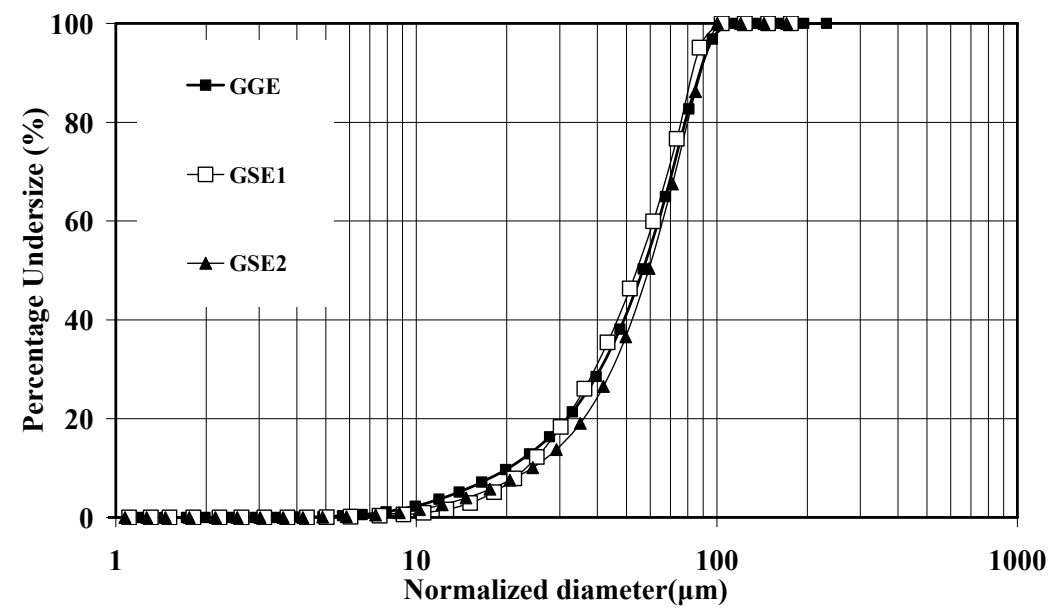

Figure 1.8-Comparison of cumulative PSD profiles normalized with the yield of the polymer extracted from three experiments

\subsubsection{The Influence of Hydrogen}

Figure 1.9 shows the rate-time profiles for two gas phase and two slurry experiments. In the slurry phase, increasing the hydrogen partial pressure leads to an increasing polymerization rate, whereas in the gas phase the polymerization rate decrease dramatically in the presence of hydrogen. 


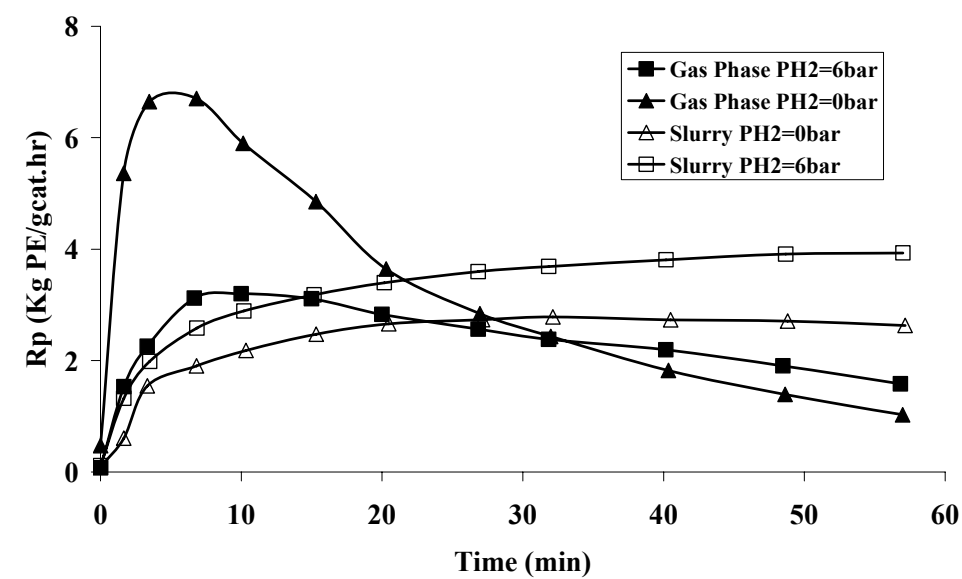

Figure 1.9-Hydrogen Influence on Slurry and gas-phase Polymerization at $\mathrm{T}=80^{\circ} \mathrm{C}$ and $\mathrm{P}_{\mathrm{C} 2}=2$

bars.

Figure 1.10 represents PSD profiles normalized with the yield of hydrogen series experiments. Particle size distribution mesurements shows that fines generation in slurry polymerization due to the hydrogen introducing is much more dramatic than fines generation in the gas-phase polymerization. The difference in the reaction rates between the slurry and gas phase has never been satisfactorily described nor explained (???). However, this phenomenon will be explained in this thesis.

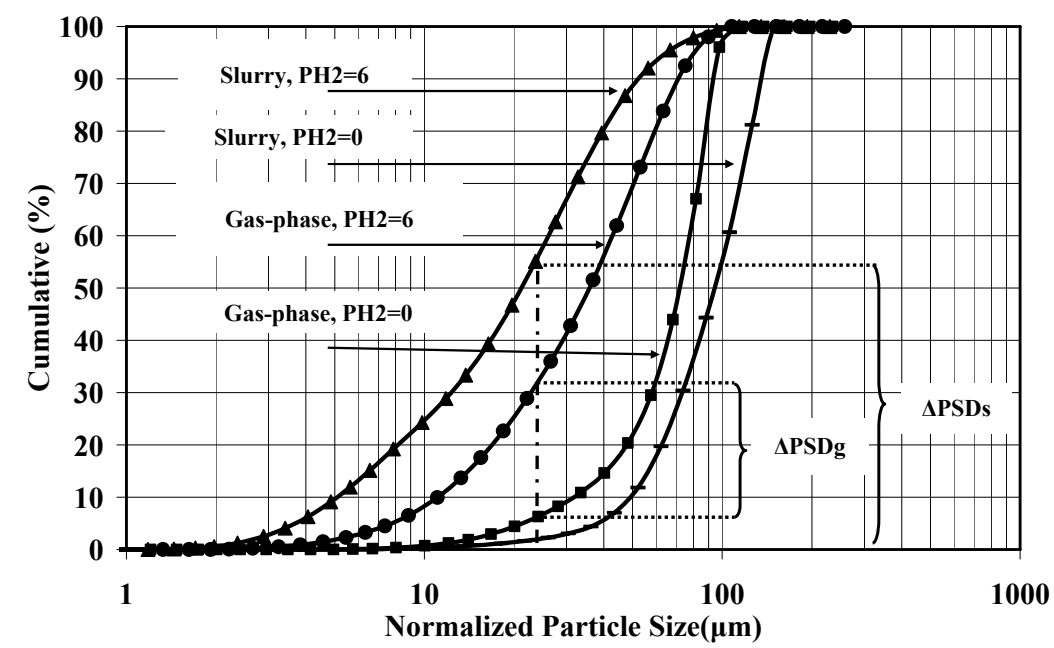

Figure 1.10-Hydrogen influence on cumulative PSD profiles normalized with the yield on slurry and gas-phase ethylene polymerization at $\mathrm{T}=80^{\circ} \mathrm{C}$ and $\mathrm{P}_{\mathrm{C} 2}=2$ bars.

\subsection{Research Statement}

This research is intended to compare slurry and gas-phase polymerization processes in single and multi-step reaction for HDPE. It will look at the influence of polymerization 
parameters such as co-catalyst concentration, temperature, ethylene partial pressure, hydrogen partial pressure and solvent participation on the reaction rate profile as well as the particle size distribution, molecular weight distribution and crystallinity of the produced powder, and in some special cases by TEM and SEM pictures.

As we will see in the following chapters, a number of phenomena even in the simplest case of the homo-polymerization of ethylene cannot be explained based on the knowledge published so far. Starting with an idea regarding the interaction between particle disintegration and polymerization rate profile, a hypothesis was developed that can explain most of the "strange" phenomena found in a large number of experiments that are described in the following chapters. During the last 4 years, we developed this GRAF hypothesis, i.e. Growth Rate Acceleration by Fragmentation, as a useful tool for interpreting ethylene polymerization experiments taking into account the interaction of kinetics and morphology.

\subsection{Thesis Goals}

The goals of this thesis follow from the fundamental statement that the actual concentration of rate-and-property determining components (for instance, ethylene, hydrogen, and co-catalyst) near the active centres of the catalyst plays an important role in the kinetics of polymerization reactions $[47,48]$ as well as in the morphology of powder produced by Ziegler-Natta catalysts. Most important is the mechanism of active sites generation as a consequence of the catalyst particle fragmentation, which is seen in GRAF as a two-step process: first, new sites are generated by fragmentation, which are not active in-situ, but can be activated by the co-catalyst in a second step. This activation step depends on the microconditions around these potentially active sites. This behaviour is highly influenced by many variables, such as temperature, ethylene partial pressure, hydrogen partial pressure, cocatalyst concentration and solvent participation. Therefore, this thesis will describe, compare and explain the influences of these variables on the reaction rate profile, particle growth, and MWD of powder produced in one and multi-step slurry and gas-phase ethylene polymerization processes.

\subsection{Thesis Outline}

This thesis will comprehensively compare slurry and gas-phase homo-ethylene polymerization using Ziegler-Natta catalyst. It consists of eight chapters.

Chapter 1 presents the general introduction including the types of polyethylenes, polyethylene technologies, and focussing more on slurry and gas-phase processes by describing a typical example of each process. Next, the general advantages and drawbacks of slurry and gas-phase polymerization processes are discussed. Then a comparison of slurry and gas-phase polymerization, especially in micro-level, from academic point of view is made. Finally, the research statement, the main objectives and the thesis goals are described. 
Chapter 2 describes the detail of the experimental set-up used in this project, the methods applied for characterization of polymer produced in each experiment and the modelling methods for the prediction of component composition in the slurry and gas-phase experiments. We also describe the deconvolution analysis, which is one of the most successful methods for calculating the fraction, the number of flory components and their influence on molecular weight distribution.

Chapter 3 presents the basic results achieved in the slurry and gas-phase polymerization experiments. To be sure that the reproducibility of experiments is acceptable, a series of repeating experiments in slurry was performed. In addition, systematic experiments moving from gas-phase towards slurry by introducing different amounts of n-hexane have been performed to analyze the rate profile and the properties of the obtained polymers. Furthermore, the influence of the pre-contacting time of the catalyst with co-catalyst on the reaction rate of slurry polymerization and the properties of polymer produced in corresponding experiments are discussed. The replication phenomenon that occurs during the course of reaction is investigated by performing several reproducible experiments over various reaction times. Finally, the influence of nitrogen partial pressure on the reaction rate profile is also examined.

Chapter 4 describes the kinetic influence of the reaction temperature on the reaction rate profiles of slurry and gas-phase ethylene polymerization both with and without the presence of hydrogen as discovered by conducting a series of experiments at different temperatures. The activation-deactivation behaviour of the catalyst, $K_{d}$ (deactivation

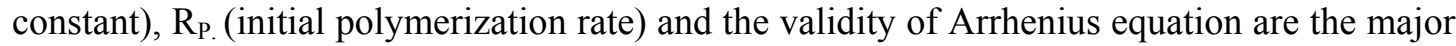
topics that are presented in this chapter. In terms of product characterization, the influence of reaction temperature on molecular weight $(\mathrm{Mw}, \mathrm{Mn}, \ldots)$, molecular weight distribution (MWD), crystallinity and particle size distribution of polymer produced are described.

Chapter 5: Nothing appears in the literature, about a comprehensive comparison of the influence of ethylene partial pressure on the reaction rate profiles of slurry and gas-phase ethylene polymerization and its interaction with properties of produced polymer. Therefore, the investigation described in this chapter was carried out to compare, identify and evaluate precisely the influence of ethylene partial pressure on slurry and gas-phase ethylene homopolymerization on the kinetics, MWD, and crystallinity of produced polymer, and their interactions with particle fragmentation and disintegration (fines and agglomerates generation) for Ziegler-Natta catalyst systems.

Chapter 6 presents a comparison between catalytic slurry and gas-phase ethylene polymerization processes on the basis of the hydrogen influence on polymerization kinetics, molecular weight, crystallinity and particle size distribution. This chapter also describes how hydrogen as a chain transfer agent can significantly affect the reaction rate of slurry polymerization (showing a rate-enhancement effect) compared to gas-phase polymerization 
(showing a rate-retarding effect) for a given Ziegler-Natta catalyst system. In this chapter, we discuss the "in-situ polymer mobility of short chains" as a substantial part of GRAF being an important phenomenon affecting the reaction rate profiles and fines production in the presence of hydrogen.

Chapter 7 presents a description of two-step polymerizations to study the role of brittle and ductile PE within the GRAF interpretation. It discusses various configurations of slurry and gas-phase two-step reaction for improving the particle size distribution of the produced polymer. By performing a series of two-step reactions with different combinations of slurry and gas-phase, we show under which conditions fines are produced and how industry can reduce the fines generation, which is one of the most important problems in industrial polyolefin production.

Chapter 8 summarizes this thesis, and includes both our conclusions and our recommendations for future research. 



\section{Chapter 2}

\section{Experimental and Theoretical Methods}

\subsection{Experimental Procedure}

\subsubsection{Reactor}

The experimental set-up for slurry and gas-phase polymerizations is shown schematically in Figure 2.1. The set-up was designed for ethylene (co-) polymerization in both slurry and gas-phase and has been described in detail by M.F.Bergstra [45].

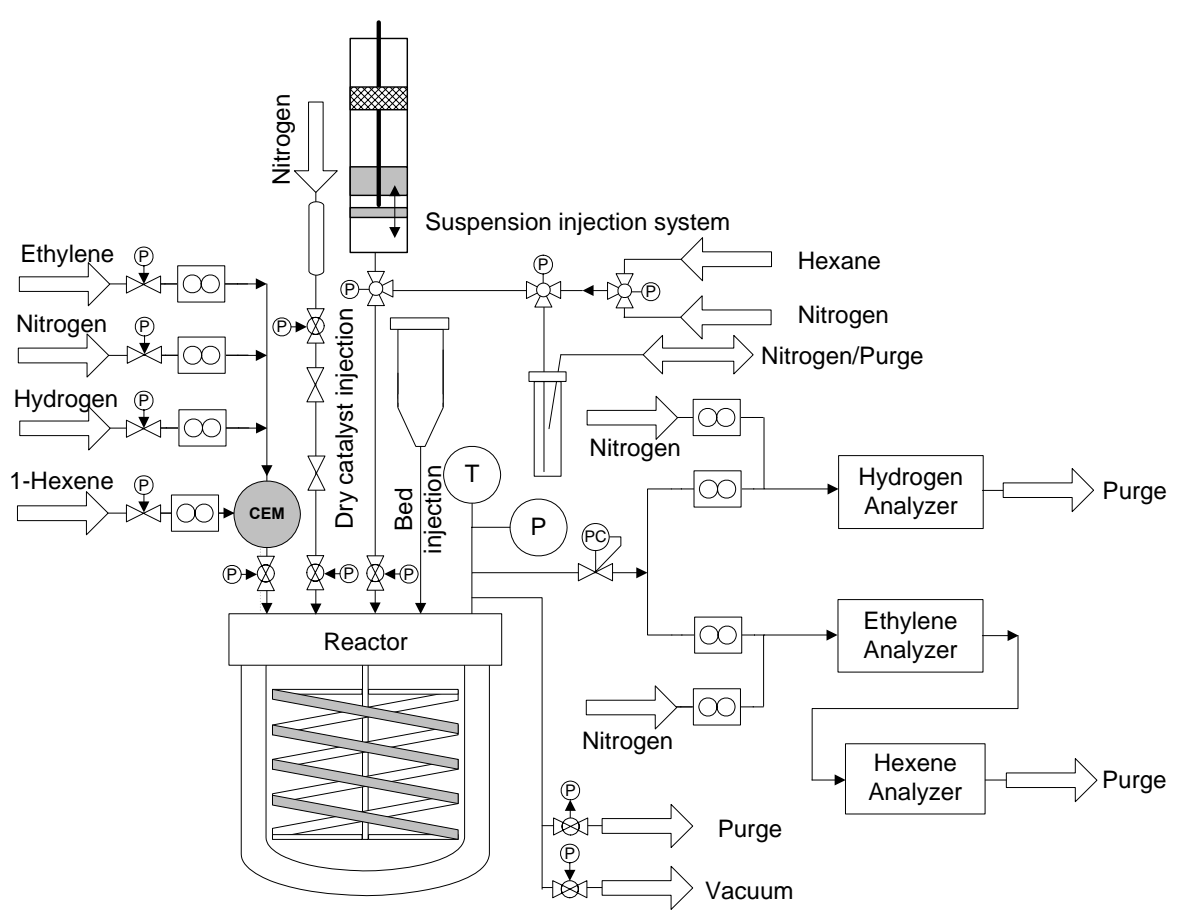

Figure 2.1-A schematic representation of the experimental set up taken from [45]

The reactor is a 1.6- $\mathrm{L}$ stainless steel jacketed vessel from Büchi that can be operated at pressures up to 40 bar and temperatures up to $120^{\circ} \mathrm{C}$. The set-up is equipped with two automatic catalyst injection systems for dry powder, suspended catalyst (in hexane or oil) and co-catalyst. Catalyst can be either injected as a dry powder via a dry 
injection system or as a suspended catalyst (in hexane or oil) via a wet system. For gas phase polymerizations, the seedbed can be injected using a bed injection vessel. Solvent for the slurry experiment can be fed in either by means of the slurry injection system or directly via a liquid mass flow meter.

A helical stirrer is used to achieve better mixing of components and better heat transfer through the cooling jacket [49]. The stirrer speed can be varied up to $2000 \mathrm{rpm}$. All gases are fed by thermal mass flow meters. The thermocouple for measuring the temperature of the reaction is located above the helical stirrer, and is in contact with the circulating reaction mass. The reaction temperature and pressure can be adjusted and controlled in the isothermal (within $0.2 \mathrm{C}$ ) and isobaric (within 0.15 bar) modes. All pressures, temperatures and mass flows are measured by a Data Acquisition/Control Unit (HP 3852A) and are stored on a PC (Agilent-VEE software). The ethylene flow under isothermal-isobaric conditions represents for the polymerization rate.

\subsubsection{Catalyst Handling and Preparation}

A hetrogeneous Ziegler-Natta catalyst system, kindly provided by an industrial partner, was used in this project. $\mathrm{Cg}$ represents for a typical gas phase polymerization catalyst, used in industries. Figure 2.2 shows the differential and integral particle size distibution of the catalyst. This catalyst, with an average particle size of around $60 \mu \mathrm{m}$, consisted of a spherical $\mathrm{MgCl} 2-\mathrm{EtOH}$ support which was titanated with titanium chloride. (see Figure 2.3) which has been titanated with titanium chloride. The catalyst was stored and handled as a dry powder in a Braun 150 B-G-H glove box under nitrogen atmosphere. The glove box was equipped with gas analyzer, which can show any changes in impurity within the box of more than $1.0 \mathrm{ppm} \mathrm{O} 2$ and $\mathrm{H} 2 \mathrm{O}$.

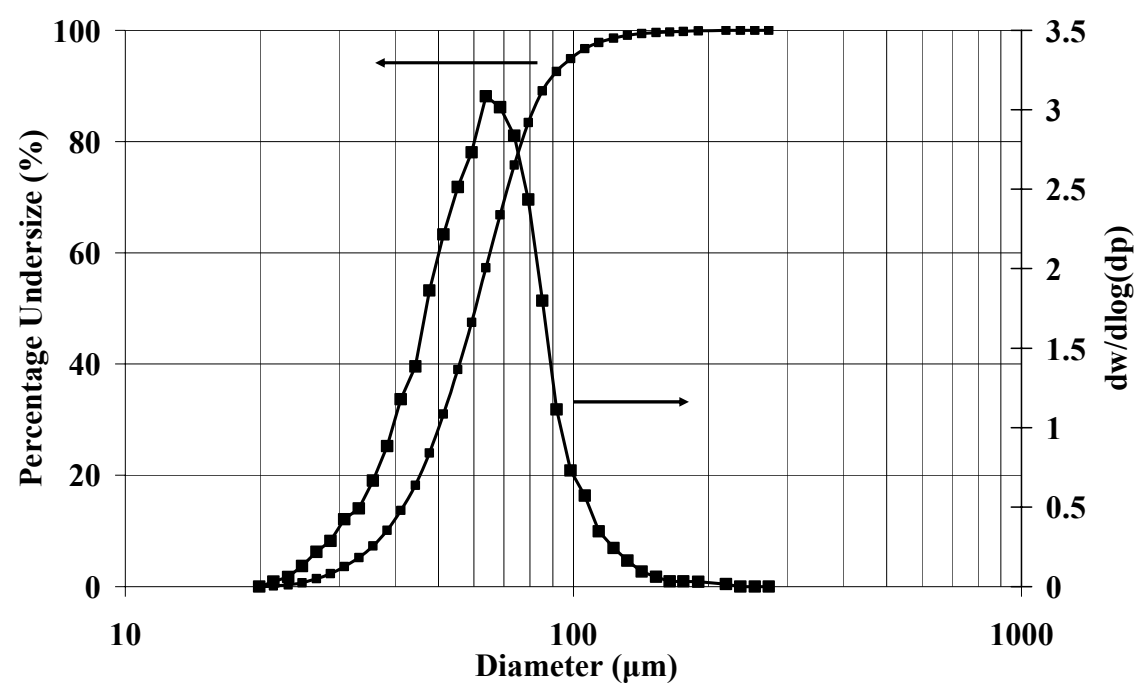

Figure 2.2-Comparison of particle size distribution of $C_{g}$ Catalyst 
$\mathrm{Cg}$ was pre-contacted with triisobutyl aluminum (TIBA)-from Akzo Nobel- as a co-catalyst and then diluted with purified $n$-hexane for approximately 30 minutes. TIBA was also used as scavenger, prior to introducing the catalyst mixture into the reactor.

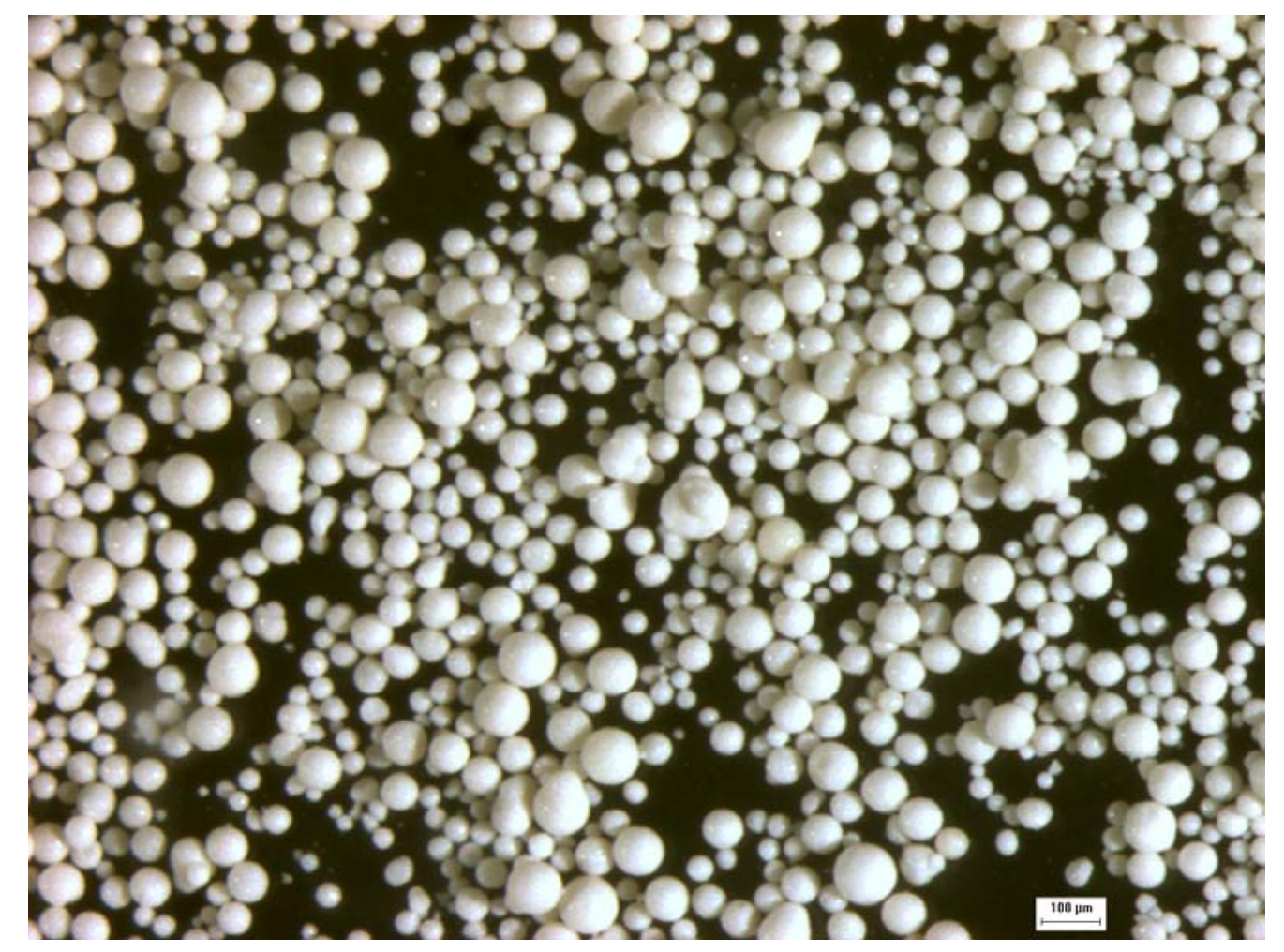

Figure 2.3-The picture of hetrogeneous Ziegler-Natta catalyst $C_{g}$

\subsubsection{Seedbed Preparation}

Sodium chloride is used as seedbed for gas-phase ethylene homopolymerization, because

(1) it is chemically inert

(2) it improves mixing and therfore helps to avoid catalyst agglomeration [18]

(3) polymer and salt mixture can be easily separated by washing with water.

The salt was sieved (between 200 and 500 microns) and dried at $280^{\circ} \mathrm{C}$ under vacuum for two days. We used exactly $110 \mathrm{~g}$ salt for each experiment.

\subsubsection{Gas-Liquid Purification}

All gases and liquids used were of 'polymer grade'. Because of the high sensitivity of Ziegler-Natta catalyst to impurities and dramatic poison influences on the reaction rate 
and the reproducibility of the polymerization experiments, all gases and liquids were purified in a series of purification columns before use.

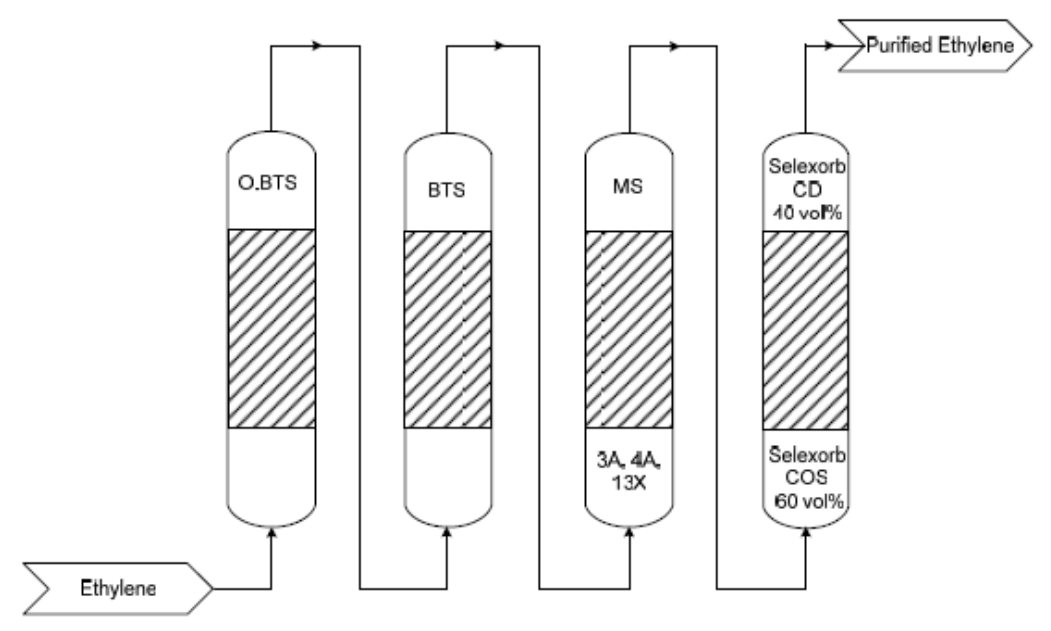

Figure 2.4-Purification scheme for ethylene

Ethylene with a purity $>99.9 \%$, obtained from Hoekloos, was used in this study. It was further purified by passing through four purification columns (shown in Figure 2.4). These columns consist of different catalysts and molecular sieves.

- oxidized BASF R3-16 catalyst for oxidizing $\mathrm{CO}$ to $\mathrm{CO}_{2}$

- reduced BASF R3-16 catalyst to chemically absorb oxygen

- molecular sieves (3A, 4A, 13X, obtained from Sigma-Aldrich) to physically absorb $\mathrm{CO}_{2}, \mathrm{H}_{2} \mathrm{O}$ and other impurities

- 50-50 combination of SelexsorbR COS (Alcoa) for removing $\mathrm{COS}, \mathrm{H}_{2} \mathrm{~S}, \mathrm{PH}_{3}$, and Selexsorb CD (Alco) for removing oxygenates (for example, ethers, alcohols, aldehydes, carbonyls, ketones, peroxides).

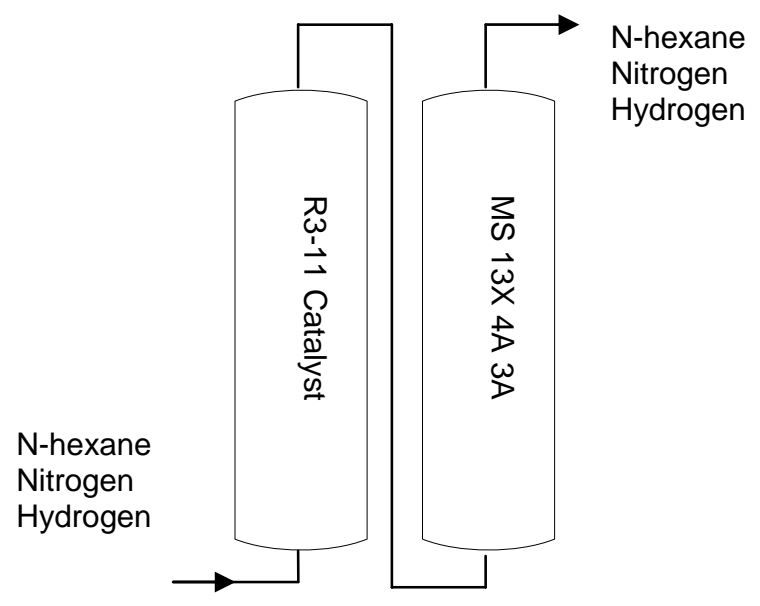

Figure 2.5-Purification scheme for $n$-hexane, nitrogen and hydrogen 
Hydrogen (purity $>99.999 \%$, Hoekloos), nitrogen (purity $>99.998 \%$, Paraxair) and n-hexane (purity $>99 \%$, pro synthesis, Merck) were also purified through the separate purified equipments. Those included two columns, reduced BASF 3R-11 catalysts to chemically absorb oxygen and molecular sieves (3A, 4A, 13X, Sigma-Aldrich) to physically absorb $\mathrm{CO}_{2}, \mathrm{H}_{2} 0$ and other impurities, respectively (see Figure 2.5).

\subsubsection{Polymerization Procedures}

1. Inspect the reactor to be certain that there was no trace of dirt or remnants of the previous experiments.

2. Close the reactor and carry out a leak check by pressurizing with nitrogen up to 20 bar. The pressure loss mus be smaller than $0.5 \mathrm{bar} / \mathrm{h}$ (about $1 \mathrm{~g} / \mathrm{h}$ loss).

3. Heat the reactor up to $90^{\circ} \mathrm{C}$ followed by 10 times pressurizing with purified nitrogen, purging, and evacuation especially to remove oxygen and moisture.

4. For gas-phase polymerization, introduce salt into the reactor by means of the seedbed injection unit while the mixer is off and the reactor remains under vacuum. For the slurry reaction, introduce hexane.

5. Pressurize with 2 bar nitrogen and mix the reactor content at 200 RPM for 2 minutes.

6. Inject the scavenger (TIBA mixed with $1 \mathrm{ml}$ Hexane in a $3 \mathrm{ml}$ vial) by means of pneumatic injection. Allow it to mix for 10 minutes.

7. Set the reactor temperature and feed hydrogen and ethylene to desired set point; wait until temperature and pressure fix.

8. Inject the catalyst mixture.

9. Fix a new pressure set point (the pressure increases after catalyst injection).

10. The reaction is automatically controlled by special software; all data are continuously saved to a PC.

11. At the end of each experiment, close all feed valves, open the purging valve and set the reactor heating to "off" for rapid depressurizing and cooling down.

12. Purge the reactor with nitrogen to remove monomer and hydrogen

13. Open the reactor and collect the polymer for analysis.

\subsection{Estimation of Reaction Rate}

The reaction rate during the course of a reaction is measured under isothermalisobaric conditions by measuring the instantaneous monomer mass flow that is introduced to 
the reactor to keep the pressure constant. Following the common standard, corrections for monomer solved in the changing polymer and/or liquid phase are not made.

Whenever in this thesis, we use the rate of polymerization $R_{p}$, this means kilograms of polymer produced per gram of supported catalyst per hour of reaction time, expressed by means of the following equation:

$$
R_{p}=\frac{W_{p}}{W_{c a t} . t}
$$

where $W_{p}$, is the weight of produced polymer in kilogram during reaction, $W_{c a t}$ is the weight of used supported catalyst in gram and $t$ is the duration of reaction in hours.

\subsection{Particle Size Distribution Measurements and Analyses}

To achieve reliable and accurate PS and PSD data for the polymer produced in all experiments, two different methods are used in this study. First, whole powder produced was weighed and screened for 15 minutes by using an electric Sieve Shaker that had sieves with mesh sizes between 3000 and 38 micron. Second, the sieved powder was collected and remixed. Three samples, of about 3 grams each, were analysed by using a Sympatic laser diffraction particle size analyzer (LDPSA).

For comparing the particle size and PSD of powder produced in different experiments, two methods can be applied. In the first method, experiments are stopped once the desired yield is achieved. So the PSD measured by LDPSA or sieving can be directly used and compared. However, it is hard to stop two different experiments at exactly the same yield. In the second method, the PSD is normalized with the yield as follows [50]. By assuming that:

- no fines or agglomerates are formed and

- the density and porosity of polymer and catalyst carrier do not change during the reaction

then the normalized polymer particle diameter can be derived by rearranging equation 2.2 to equation 2.3

$$
\begin{aligned}
& \frac{d_{p o l}}{d_{c a t}}=\sqrt[3]{\frac{\rho_{c a t}\left(1-\varepsilon_{c a t}\right)}{\rho_{p o l}\left(1-\varepsilon_{p o l}\right)}\left[\int_{0}^{t} R_{P} d t+1\right]} \\
& \frac{d_{p o l}}{d_{c a t}} \approx b \sqrt[3]{[Y+1]} \\
& d_{p o l} \text { - diameter of the polymer particle }(\mu \mathrm{m}), \\
& \rho_{p o l} \text { - density of polymer }\left(\mathrm{kg} / \mathrm{m}^{3}\right), \\
& \varepsilon_{p o l} \text { - porosity of polymer, } \\
& d_{c a t} \text { - catalyst diameter }(\mu \mathrm{m}), \\
& \rho_{c a t} \text {-density of catalyst (support) }\left(\mathrm{kg} / \mathrm{m}^{3}\right),
\end{aligned}
$$


$\varepsilon_{\text {cat }}$ - porosity of catalyst,

$R_{p}$ - polymerisation rate $\left(g_{\text {polymer }} / g\right.$-cat $\left.h r\right)$,

$\mathrm{Y}$ - yield of polymer $\left(g_{\text {polymer }} / \mathrm{g}\right.$ - $\left.c a t\right)$

In other words, we assume $b$ is constant and that the normalized diameter can be calculated by:

$$
d_{p o l, n}=\frac{d_{p o l}}{\sqrt[3]{[Y+1]}}
$$

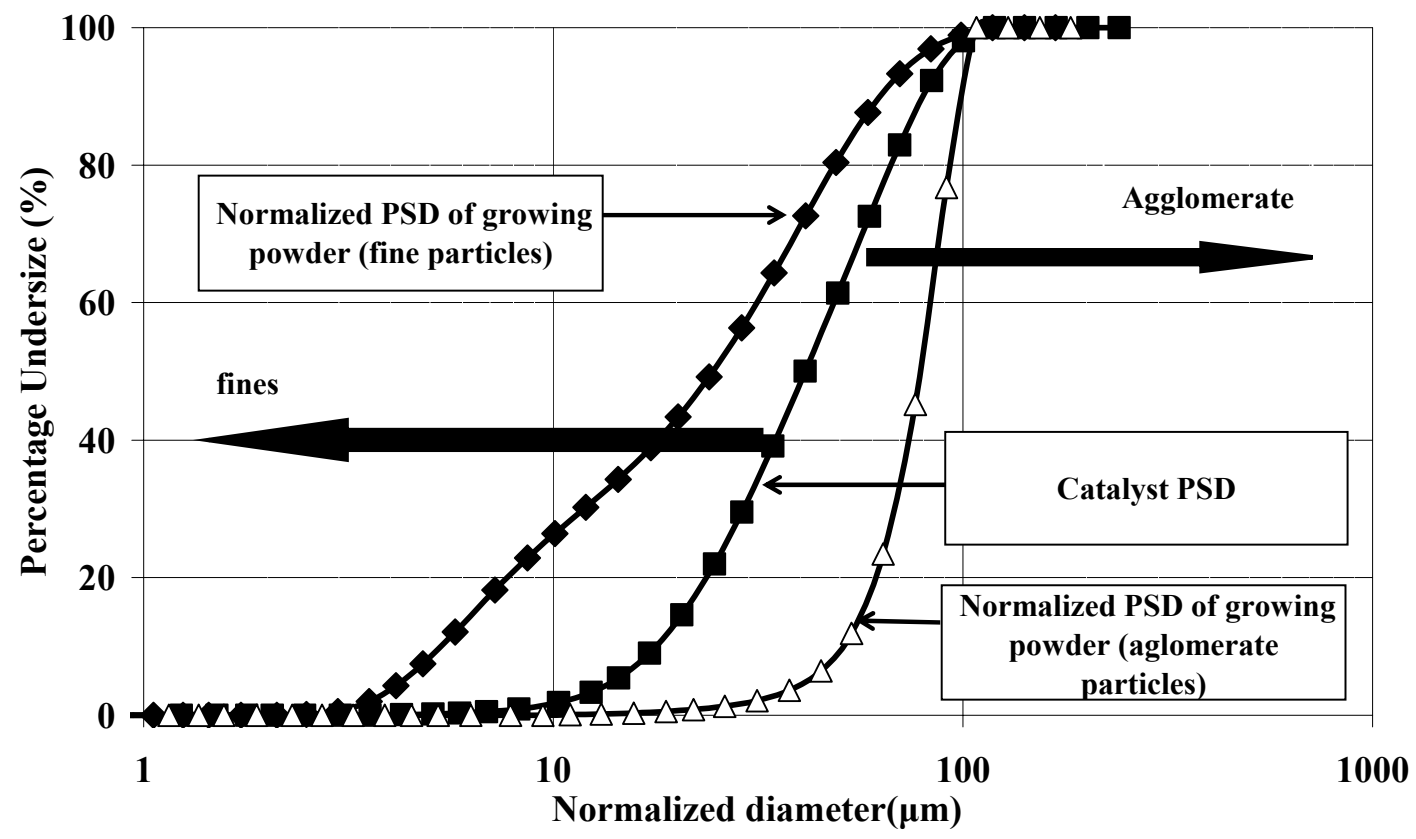

Figure 2.6 Particle size distribution of three samples

For illustration, three profiles are presented in Figure 2.6: the middle profile shows the catalyst PSD profile of the polymer product if perfect replication occurs. The left-hand profile shows a PSD of the resulting polymer if fines are generated and - in contrast - the right-hand profile shows the PSD with agglomerates.

However, in this study, we used only the normalized PSD profiles due to simplicity and accuracy of method.

\subsection{DSC Results}

A Mettler Toledo 822e STARe version 8.01 software was used $^{1}$ as follows:

- $\quad 5$ to $10 \mathrm{mg}$ samples of polymer are used

- $\quad$ keep the temperature at $30{ }^{\circ} \mathrm{C}$ for $2 \mathrm{~min}$

\footnotetext{
${ }^{1}$ I wish to thank Mrs. Zare from the National Petrochemical Company-Research and Technology, Tehran, I.R.Iran.
} 
- heat from $30{ }^{\circ} \mathrm{C}$ to $200{ }^{\circ} \mathrm{C}$ at $10 \mathrm{C} / \mathrm{min}$; keep for $2.0 \mathrm{~min}$ at $200{ }^{\circ} \mathrm{C}$

- cool to $30^{\circ} \mathrm{C}$ at $10{ }^{\circ} \mathrm{C} / \mathrm{min}$

- repeat the above heating and cooling cycle

The crystallinity is calculated by applying equation 2.4 where $\Delta \mathrm{H}_{f s}$ is the measured enthalpy of melting of the sample and $\Delta \mathrm{H}_{f p}$ is the enthalpy of crystalline polyethylene, which is equal to $269.69 \mathrm{~J} / \mathrm{g}$. The temperature assigned for the maximum heat flow is taken as melting temperature:

$$
\mathrm{X}_{\mathrm{C}}=100 . \Delta \mathrm{H}_{f s} / \Delta \mathrm{H}_{f P}
$$

The crystallinity during the first heating-cooling cycle gives information about insitu crystalliztion during the polymerization, whereas the second heating-cooling cycle provides information after recrystallization - which is often used in industry without taking care of the first cycle.

\subsection{Molecular Weight Distribution}

The GPC used in this work is a high-temperature WATERS $150 \mathrm{C}$ working at $140^{\circ} \mathrm{C}$, calibrated by standard polystyrene polymer, and using 1,2,4-TCB as a solvent and 2.6 di-tertbutyl-4-methylphenol as a stabilizer ${ }^{1}$.

\subsection{Scanning electron microscopy (SEM)}

Scanning electron microscopy (SEM) was performed using a Philips ESEM XL 30 FEG for better understanding of internal and external morphology of polymer produced ${ }^{2}$. The SEM was operating in high vacuum modus. Therefore, an Au-coating was used to prevent charging. Micrographs were taken at $5 \mathrm{kV}$ electron beam energy.

\subsection{Transmission Electron Microscopy (TEM)}

Transmission electron microscopy (TEM) was performed using a JEM 2010 JEOL microscope at a $200 \mathrm{kV}$ accelerating voltage so as to get information about the crystal

\footnotetext{
${ }^{1}$ I wish to thank Mr. A. P. Jekel from Groningen University for the Measurements

${ }^{2}$ I wish to thank Mr. V. Seydewitz from Department of Physics of Halle University. The SEM used was a device of the Interdisciplinary Center of Materials Science of Halle University
} 
structure and their distribution inside the produced polymers ${ }^{1}$. The specimens were sectioned by ultra-microtome. The contrast of density differences in the organic material (e.g. density differences between amorphous and crystalline part of a lamella) was increased by applying $\mathrm{RuO} 4$ staining.

\subsection{Deconvolution Analysis}

It is widely accepted that different active sites of the Ziegler-Natta catalyst produce polymers characterized by different Flory-type MWD, which is the main reason for the broad MWD of polyolefins produced using ZN catalysts [51-53]. Deconvolution of a given MWD gives a quantitative analysis of the contribution of these different sites; see for example [53, 54].

According to Flory's most probable distribution, one can obtain the molecular weight distribution of each site type $j$ in a catalyst structure with the following equation:

$$
w_{r, j}=r \cdot \tau_{j}^{2} \cdot \exp \left(-r \tau_{j}\right)
$$

where, $j$ is the number of active site types, $w_{r, j}$ is the instantaneous weight chain length distribution of the produced polymer on active site $j$ with the chain length of $r$, and $\tau_{\mathrm{j}}$ is the chain transfer probability of the active site $j$.

The weight average molecular weight of the whole polymer can be determined by; Equation:

$$
W_{r}=\sum_{j=1}^{n} m_{j} \cdot w_{r . j}
$$

where, $W_{r}$ is the instantaneous weight chain length distribution of the whole polymer produced by all active site types, and $m_{j}$ is the mass fraction of polymer produced by active site type $j$. Mathematical modelling ${ }^{2}$ was carried out by Matlab software, in order to estimate the minimum number of Flory's distributions required for fitting the MWD measured by GPC, based on the procedure used by Soares and Hamielec [53].

The number of active centre types and their corresponding properties such as, $\tau_{j}, M_{n}$ and $\mathrm{M}_{\mathrm{w}}$ can be achieved by applying a deviation of less than $1 \mathrm{E}-5$.

Figure 2.7 shows the MWD and their deconvolution results (five-site model) of polymer produced at $\mathrm{T}=60^{\circ} \mathrm{C}, \mathrm{P}_{\mathrm{H} 2}=2$ bar and $\mathrm{P}_{\mathrm{C} 2}=2$ bar in slurry ethylene polymerization. Figure 2.8 shows the residuals of the measured distribution by GPC and the model obtained by deconvolution.

\footnotetext{
${ }^{1}$ I wish to thank Mrs. S. Goerlitz from Department of Physics of Halle University.

${ }^{2}$ I wish to thank Mr. Ali Safinejad from National Petrochemical Company-Research and Technology, Tehran, I.R.Iran.
} 


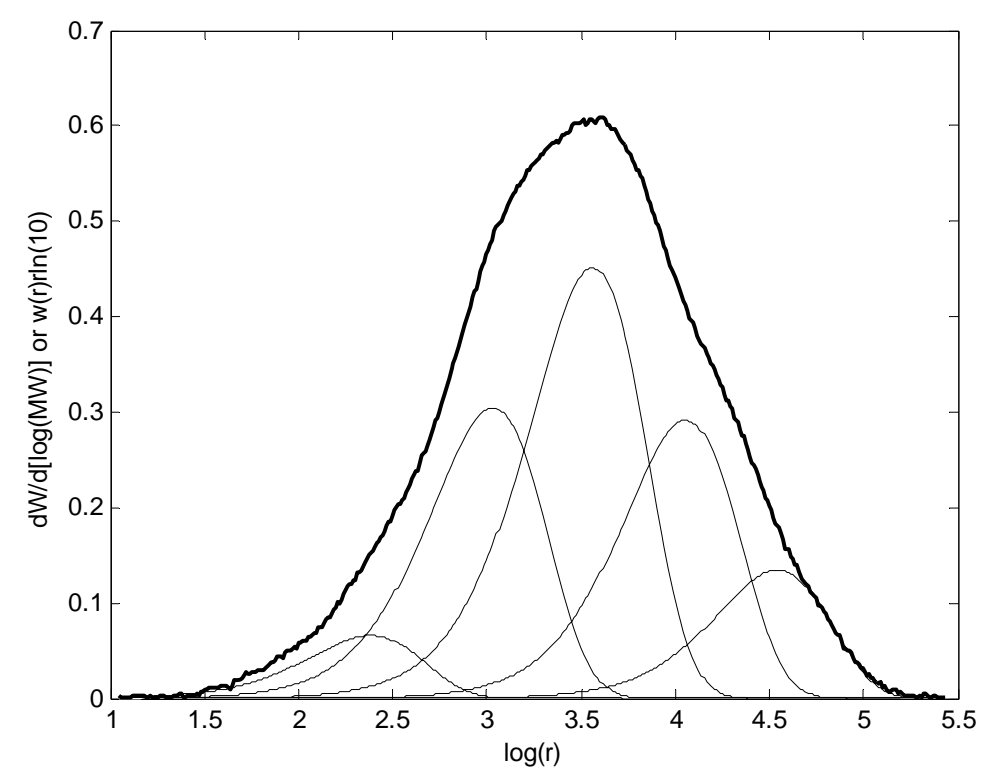

Figure 2.7 MWD obtained by GPC and deconvolution analysis of produced polymer in slurry ethylene polymerization at $\mathrm{T}=60^{\circ} \mathrm{C}, \mathrm{P}_{\mathrm{H} 2}=2$ bar and $\mathrm{P}_{\mathrm{C} 2}=2$ bar

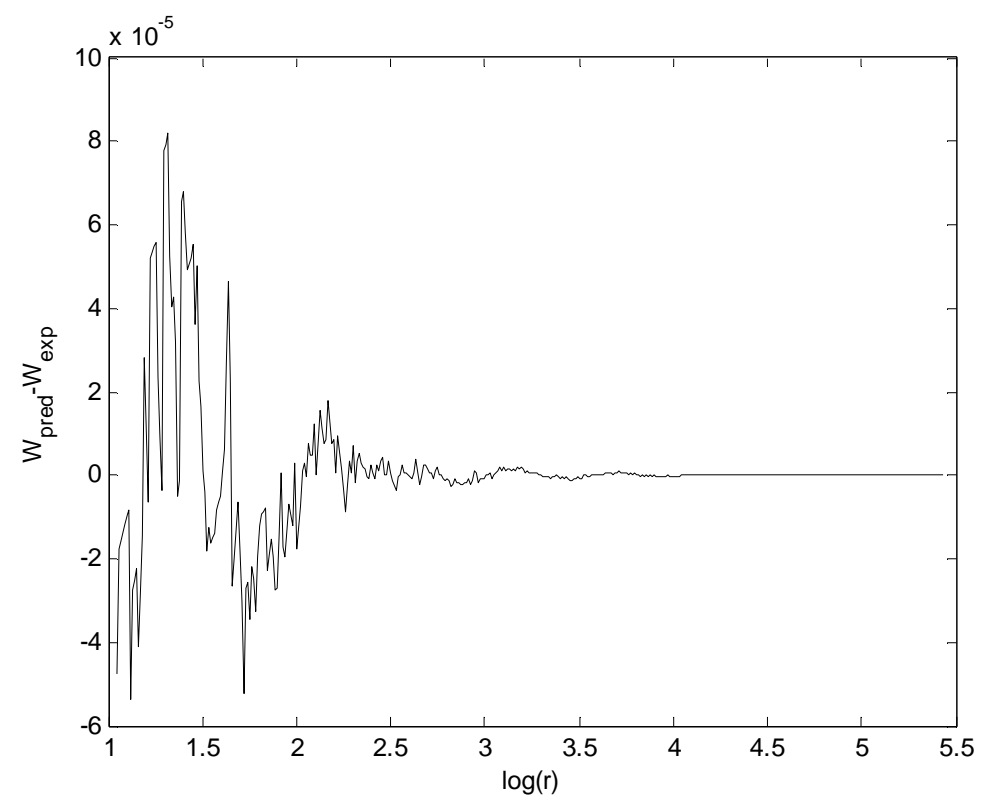

Figure 2.8 Residuals of the measured distribution and the model

\subsection{Prediction of Ethylene and Hydrogen Concentration in Slurry and Gas-phase Reactors}

To predict the concentration of ethylene and hydrogen in slurry and gas-phase experiments, the experimental procedures before reaction were modelled ${ }^{1}$ by the advanced Aspen Polymer Plus, version 11.1 software tool. The Sanchez-Lacombe and the Soave-Redlich-Kwong

${ }^{1}$ I wish to thank Mr. Ali Safinejad from the National Petrochemical Company-Research and Technology, Tehran, I.R.Iran. 
(SRK) equation of states were used for thermodynamic calculations in slurry and gas-phase experiments, respectively. In the following sub-sections, we briefly present descriptions of these two EOSs. The interested reader can find their detail descriptions in many publications, for example in [55].

\subsubsection{The Sanchez-Lacombe Equation of State Model}

Among a large number of equations of state reported in the literature for polymeric systems, the Sanchez-Lacombe equation of state (S-L EOS) is the most widely used EOS. S-L EOS is based on icing or lattice fluid theory and uses a statistical mechanical model. S-L EOS is very similar to the Flory-Huggins model, except that empty lattice sites or free volume are included in the lattice so that volume changes which are due to the mixing are predictable [56].

Sanchez and Lacombe derived the following EOS on the basis of minimizing their developed Gibbs free energy expression for pure components:

$$
\bar{\rho}^{2}+\bar{P}+\bar{T}\left[\ln (1-\bar{\rho})+\left(1-\frac{1}{r}\right) \bar{\rho}\right]=0
$$

where $r$ is the number of segments per chain, $\bar{\rho}, \bar{P}$ and $\bar{T}$ are the reduced density, pressure and temperature for pure component, respectively defined as:

$$
\bar{\rho}=\frac{\rho}{\rho^{*}} \quad \bar{P}=\frac{P}{P^{*}} \quad \bar{T}=\frac{T}{T^{*}}
$$

where $\rho, P, T$ are the actual density of the pure component, pressure and temperature of the phase, respectively. $\rho^{*}, P^{*}$ and $T^{*}$ are characteristic density, pressure and temperature related to lattice variables as follows:

$$
\rho^{*}=\frac{M}{r v^{*}}, P^{*}=\frac{\varepsilon^{*}}{v^{*}}, T^{*}=\frac{\varepsilon^{*}}{k}
$$

where: $\varepsilon^{*}$ is the characteristic interaction energy per segment, $v^{*}$ is the closed-packed volume of a segment, $M$ is the molecular weight and $k$ is Boltzmann's constant.

For fluid mixtures, equation 2.7 is used with the same parameters, except for those that need to be redefined by their mixing rules parameters as follows:

$$
\begin{aligned}
\frac{1}{r_{\text {mix }}} & =\sum_{j} \frac{\phi_{j}}{r_{j}} \\
v_{\text {mix }}^{*} & =\sum_{i} \sum_{j} \phi_{i} \phi_{j} v_{i j}^{*} \\
\varepsilon_{\text {mix }}^{*} & =\frac{1}{v_{\text {mix }}^{*}} \sum_{i} \sum_{j} \phi_{i} \phi_{j} \varepsilon_{i j}^{*} v_{i j}^{*}
\end{aligned}
$$

where: $r_{\text {mix }}, v_{\text {mix }}^{*}$ and $\varepsilon_{\text {mix }}^{*}$ are mixture parameters, $\varnothing_{i}$ is the volume fraction of component $i, v^{*}{ }_{i j}$ and $\varepsilon^{*}{ }_{i j}$ are cross-parameters as defined below: 


$$
\phi_{i}=\frac{\frac{m_{i}}{\rho_{i}^{*} v_{i}^{*}}}{\sum_{j}\left(\frac{m_{j}}{\rho_{j}^{*} v_{j}^{*}}\right)} \quad v_{i j}^{*}=\frac{1}{2}\left(v_{i i}^{*}+v_{j j}^{*}\right)\left(1-\eta_{i j}\right) \quad \varepsilon_{i j}^{*}=\sqrt{\varepsilon_{i i}^{*} \varepsilon_{j j}^{*}}\left(1-k_{i j}\right)
$$

where: $m_{i}$ is the weight fraction of component $i, \eta_{i j}$ and $k_{i j}$ are binary interaction parameters extracted from the publication Khare and co-workers [57].

\subsubsection{The Soave-Redlich-Kwong Cubic Equation of State}

Redlich and Kwong in 1949 [58] successfully proposed the following EOS for the prediction of the thermodynamic properties of the vapour phase:

$$
\begin{aligned}
& P=\frac{R T}{v-b}-\frac{\alpha}{v(v+b) \sqrt{T}} \\
& \alpha=\frac{0.42748 R^{2} T_{C}^{2.5}}{P_{C}} \\
& b=\frac{.08662 R T_{C}}{P_{C}}
\end{aligned}
$$

where, $\mathrm{R}$ is the gas constant, $\mathrm{P}$ and $\mathrm{T}$ are the pressure and temperature of the system, $T_{C}$ and $P_{C}$ are the critical temperature and pressure for the pure component respectively and $v$ is its molar volume.

The simplicity, validity and predictability of the equation, motivated several researchers to further develop those equations especially so that the a term would cover more components in an extended range of temperature and pressure. Soave's correlation proposed in 1972 [59], as written below, was specially accurate and predictable for hydrocarbon [60, 61]:

$$
\begin{aligned}
& P=\frac{R T}{v-b}-\frac{a}{v(v+b)} \\
& a=0.42748 \frac{\left(R T_{C}\right)^{2}}{P_{C}}\left[1+m\left(1-\sqrt{T_{r}}\right)\right]^{2}
\end{aligned}
$$

where $T_{r}$ is reduced temperature defined as $T_{r}=\frac{T}{T_{C}}$ and $m$ defined as:

$$
m=0.48+1.574 \omega-0.17 \omega^{2}
$$

where, $\omega$ is the acentric factor.

This approach is perfect for a pure component and a mixture of gases, but is not consistent for a VLE system-especially for the prediction of liquid molar volumes. Therefore, Aspen Polymer Plus software uses the polymer SRK EOS, which is a conjunction of SRK EOS with other correlations, for predicting of thermodynamic properties of mixtures containing polymer phase. 


\subsubsection{Initial Component Concentration Prediction}

Some results of the ASPEN calculations are presented below, because these data are fundamental to comparing gas and slurry polymerizations.

Figure 2.9 shows the results obtained for the concentration of hydrogen in hexane at different hydrogen partial pressures while keeping the temperature $\left(\mathrm{T}=80^{\circ} \mathrm{C}\right)$ and the ethylene partial pressure $\left(P_{C_{2}}=2 b a r\right)$ constant. The increase in hydrogen concentration with increasing hydrogen partial pressure is almost linear and reasonably follows Henry's law with $K_{H}=0.0148$ bar. $L / g$ as expressed below:

$$
P=K_{H} \cdot X
$$

where, $\mathrm{P}$ is the partial pressure of gas above the slurry in bar, $K_{H}$ is Henry's constant in bar. $L / g$ and $X$ is the concentration of gas in the slurry in $g / L$.

For the polymer phase solubility, Hutchinson and Ray [24] found that Henry's law is also applicable for the prediction of hydrogen and ethylene in the amorphous phase of polyethylene in the gas-phase.

Figure 2.10 shows that introducing hydrogen at a constant ethylene partial pressure has a negative synergic effect on ethylene concentration in liquid hexane - the ethylene concentration decreased from 5.2 to $4.8 \mathrm{~g} / \mathrm{L}$ as the hydrogen partial pressure increased from 2 to 20 bar.

Figure 2.11 and Figure 2.12 show the effect of temperature on the solubility of hydrogen and ethylene in hexane. The solubility of hydrogen decreases in the range 0.0277 to $0.0271 \mathrm{~g} / \mathrm{L}$ as the temperature of reactor increases in the range $40-90^{\circ} \mathrm{C}$. In the case of ethylene, a sharp decrease in the ethylene concentration can be seen as the temperature increases. Ethylene concentration in hexane decreases from 5.2 to $2.7 \mathrm{~g} / \mathrm{L}$ as a result of increasing the temperature of the reactor from 40 to $90^{\circ} \mathrm{C}$.

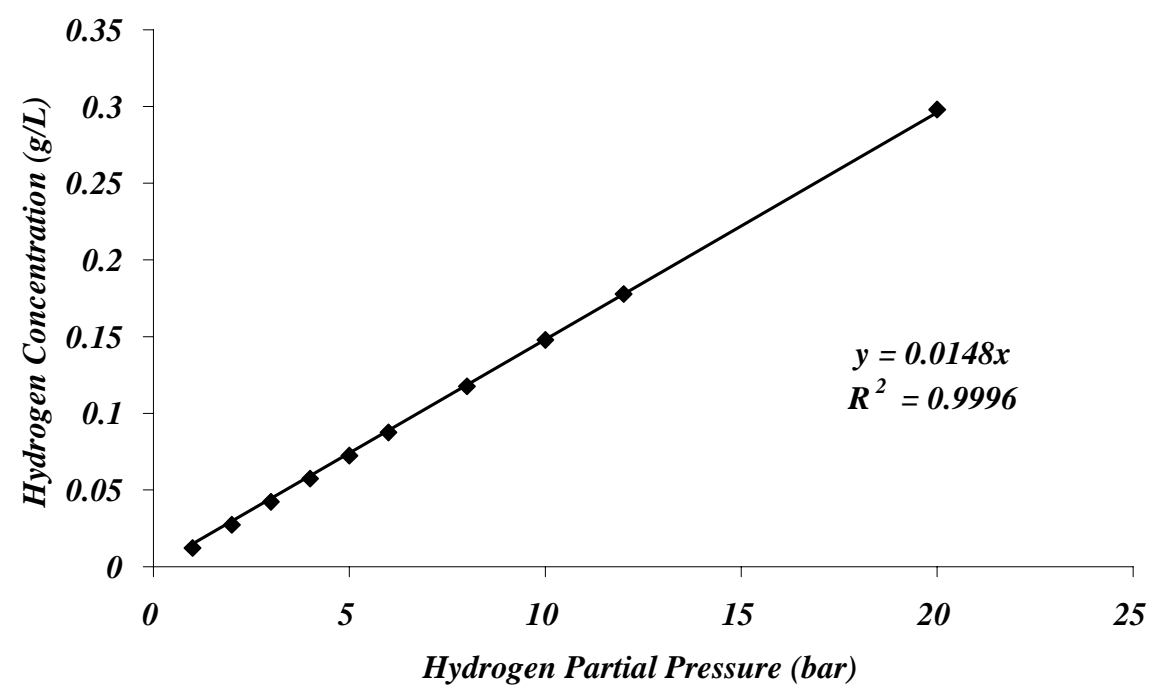

Figure 2.9-Hydrogen concentration in hexane versus hydrogen pressure at $\mathrm{T}=80^{\circ} \mathrm{C} \&$

$$
\mathbf{P}_{\mathrm{C} 2}=\mathbf{2} \text { bar }
$$




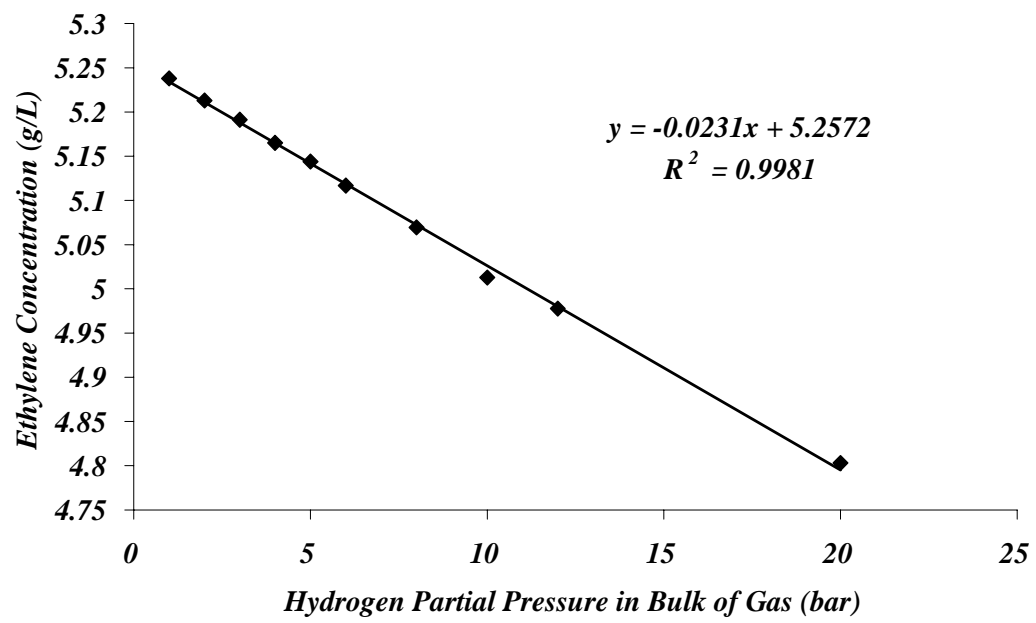

Figure 2.10-Ethylene concentration in hexane versus hydrogen pressure at $\mathrm{T}=80^{\circ} \mathrm{C} \& \mathrm{P}_{\mathrm{C} 2}=\mathbf{2}$ bar

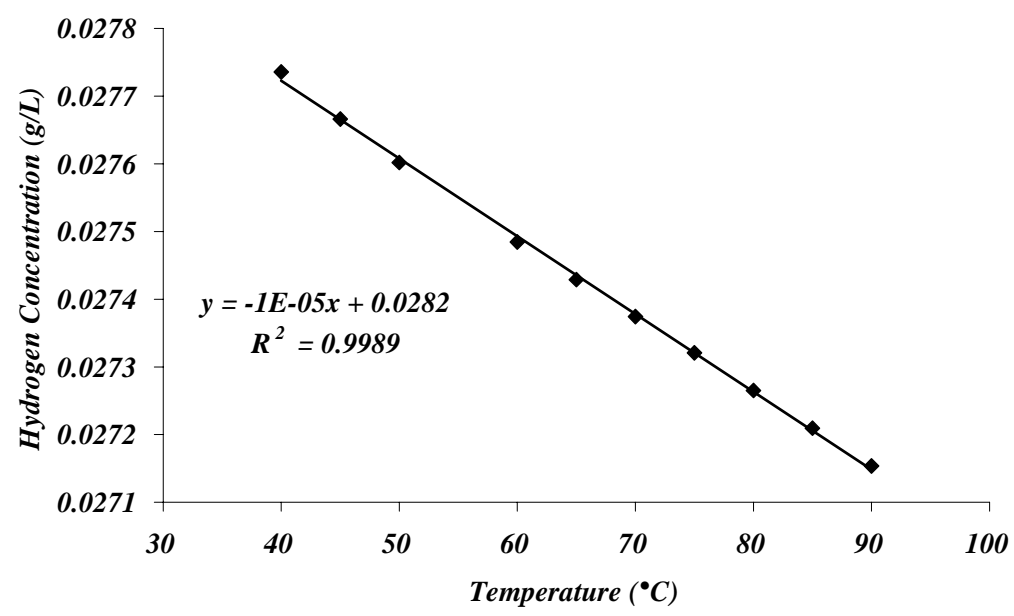

Figure 2.11-Hydrogen concentration in hexane versus temperature at $P_{\mathrm{H} 2}=2$ bar $\mathbf{P}_{\mathrm{C} 2}=2$ bar

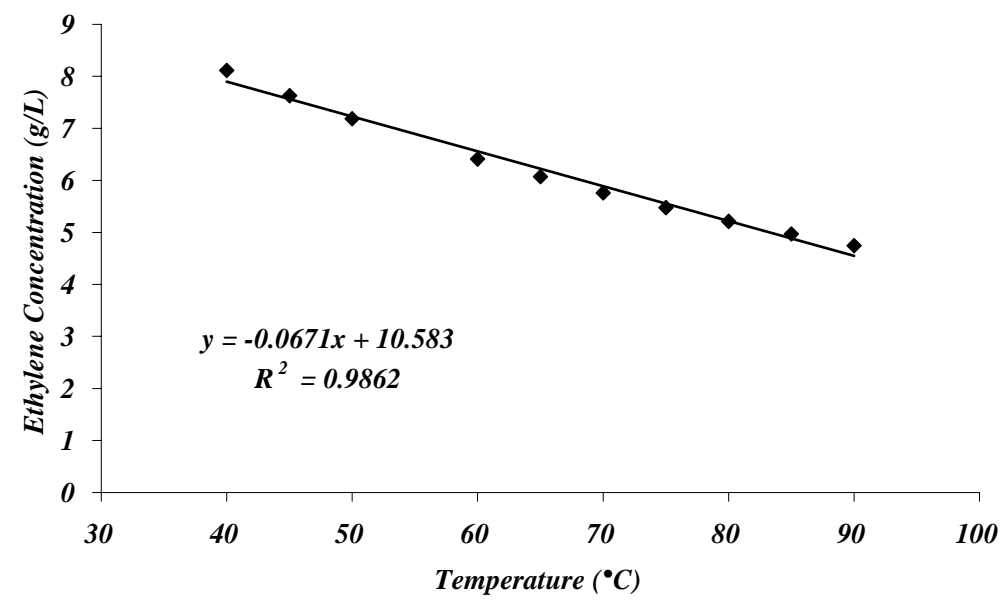

Figure 2.12-Ethylene concentration in hexane versus temperature at $\mathbf{P}_{\mathrm{H} 2}=\mathbf{2}$ bar $\mathbf{P}_{\mathrm{C} 2}=\mathbf{2}$ bar 


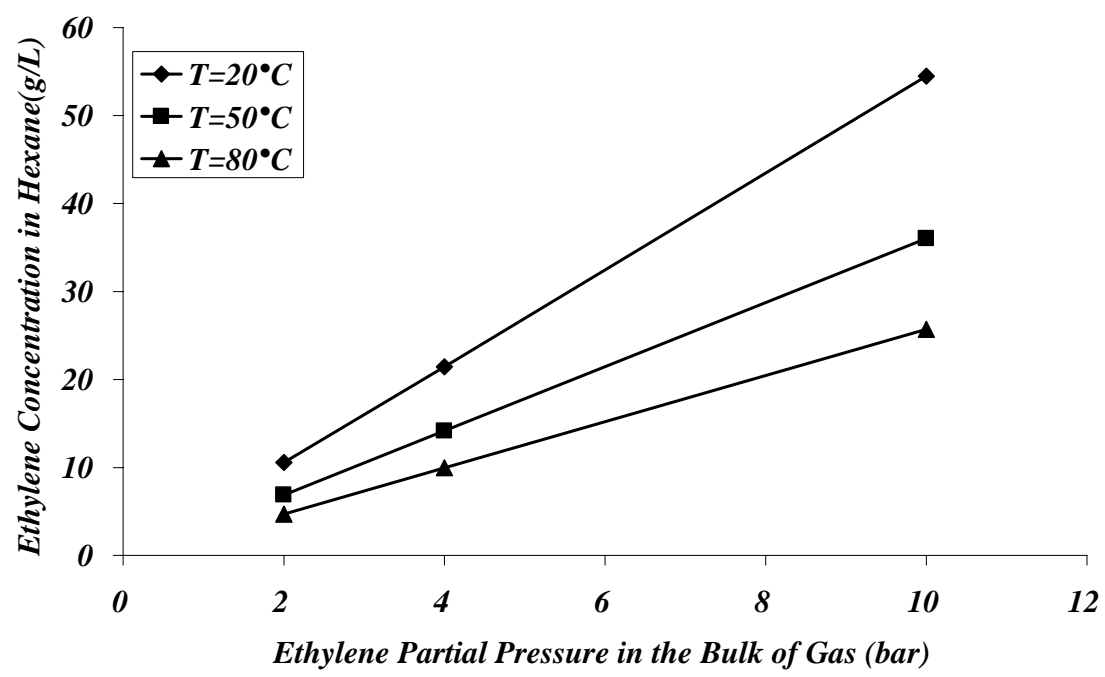

Figure 2.13-Ethylene concentration in hexane versus ethylene partial pressure at $\mathbf{P}_{\mathrm{H} 2}=\mathbf{0}$ bar Some relevant data are calculated using ASPEN for gas-phase polymerizations:

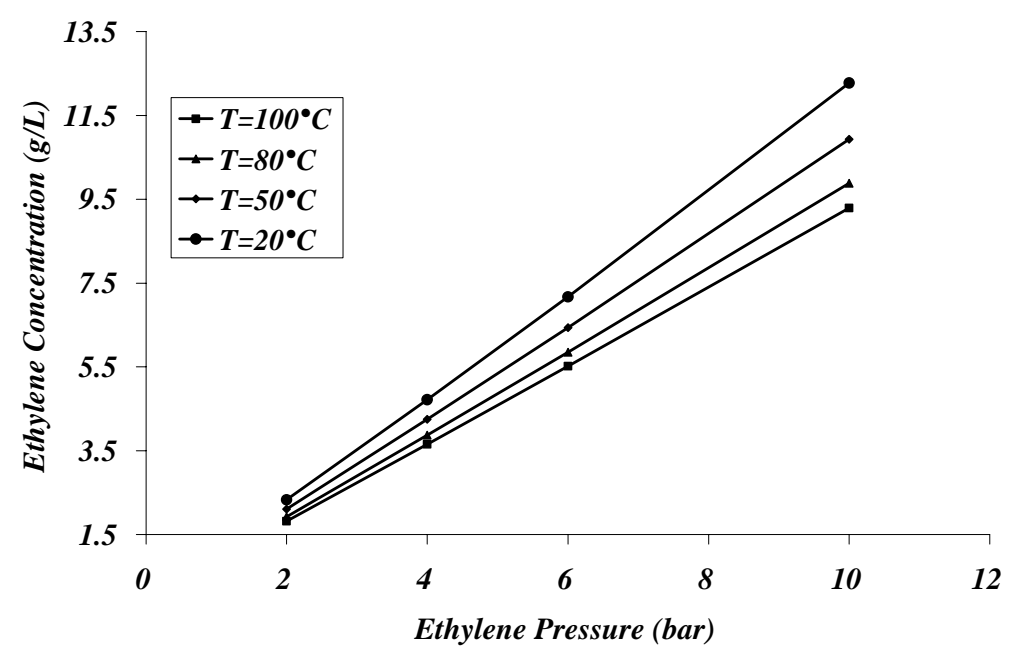

Figure 2.14-Ethylene concentration in gas-phase versus ethylene pressure at different temperature at $\mathbf{P}_{\mathrm{H} 2}=0$ bar

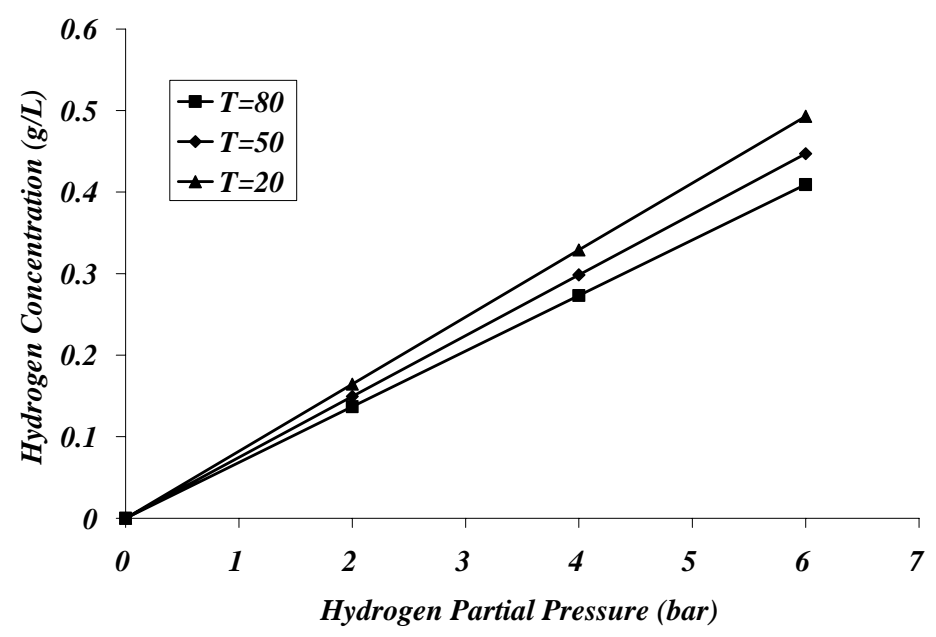

Figure 2.15-Hydrogen concentration in gas-phase versus hydrogen pressure at different temperature at $\mathbf{P}_{\mathrm{C} 2}=\mathbf{2}$ bar 
This information will enable the calculation of ethylene, hydrogen and hexane concentrations at the start of a reaction over the temperature and pressure ranges used in the experiments described in this study.

\subsection{Types of the Rate-Time Profiles}

In 1972 Keii [62] reported that most reaction rate profiles for the production of high density polyethylene followed either a build-up type or a decay type rate profile. Changing the catalyst types, precontacting and preactivation procedure of catalyst with cocatalyst, operating conditions, and the phase of reaction, can change the reaction rate profiles from one type to another[31, 32, 63]. A typical build-up type rate profile started with a rising reaction rate during the induction period, reaching a maximum followed by constant or slow rate of decay. The typical decay type rate profile started at either a maximum or with a very rapidly increasing reaction rate, followed by rapidly deactivation (see Figure 2.16).

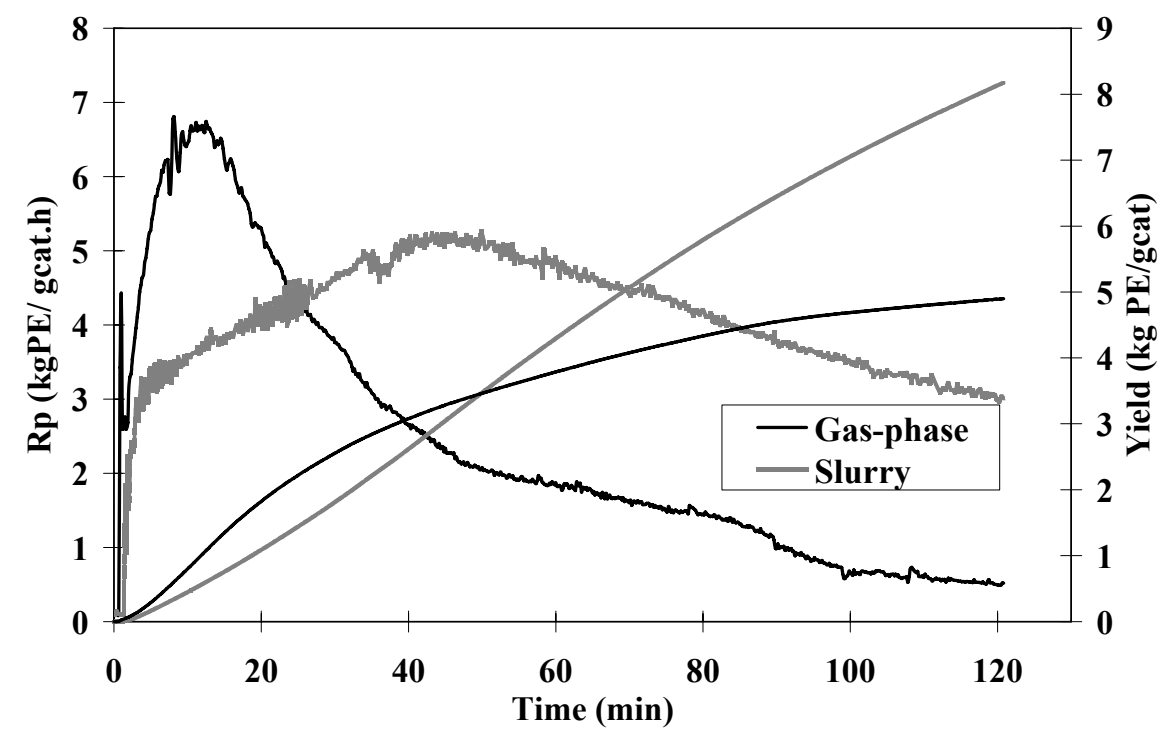

Figure 2.16-Reaction rate-profiles and their instantaneous yields in slurry and gas-phase ethylene polymerization at constant $\mathrm{T}, \mathrm{PH} 2$ and $\mathrm{PC} 2$ 


\section{Chapter 3}

\section{Basic Results}

\subsection{Introduction}

The following five series of experiments were performed using the gas-phase catalyst to find a basis within the multi-parameter, multi-dimensional space of possible experiments. The main object is to address the question: are the selected methods described in Chapter 2 suitable for working out the basic hypothesis that was defined in Chapter 1? Within which range of reaction conditions should one work?

- In the first series, the reproducibility of the experiments was checked regarding kinetics and polymer properties.

- In the second series, we moved step-by-step from pure gas-phase to pure slurry conditions by increasing the solvent quantity from $2 \mathrm{ml}$ to $700 \mathrm{~mL}$.

- The third series of experiments was performed to investigate the influence of pre-contact time on the reaction rate as well as the properties of the produced polymers.

- The fourth series of experiments was performed to investigate the PSD regarding replication phenomena: what roles do molecular weight and crystallinity play?

- The fifth series of experiments was executed to clarify the influence of an inert gas (nitrogen) on reaction rate profile and polymer properties.

\subsection{Reproducibility of Experiments}

Good reproducibility of experiments means:

- high purity of feeds and therefore good performance of all purification systems

- constant catalysts activity and therefore good quality of catalyst and co-catalyst handling and all related preparation steps

- good quality of all procedures related to reactor operation, control and data acquisition

- good probability of successful up-scaling of the results to larger reactors. 
Precise control of reaction conditions is a prerequisite for reproducibility of the experiments. All experiments have been precisely controlled to within $0.2^{\circ} \mathrm{C}$ for temperature and 0.02 bar for pressure.

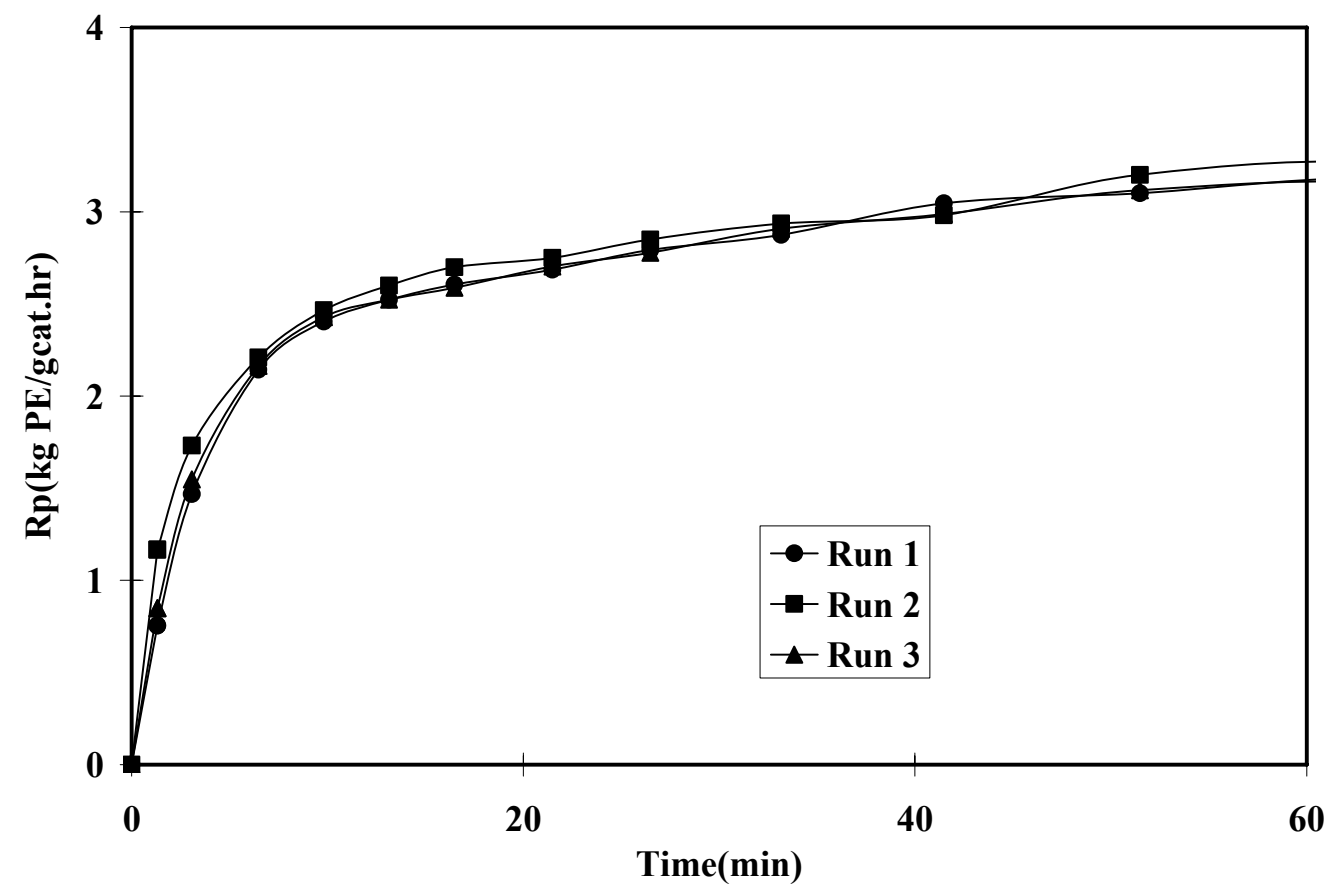

Figure 3.1-Kinetic reproducibility of three slurry ethylene polymerizations at $\mathrm{T}=80^{\circ} \mathrm{C}$ and $\mathrm{P}_{\mathrm{C} 2}=2$ bars and $\mathbf{P}_{\mathrm{H} 2}=2$ bar

Three slurry ethylene polymerizations were performed with gas-phase catalyst $(\mathrm{Cg})$ which was pre-contacted with TIBA as co-catalyst for one minute. Figure 3.1 shows polymerization rate profiles for only three experiments $\left(\mathrm{P}_{\mathrm{C} 2}=2\right.$ bars, $\mathrm{T}=80{ }^{\circ} \mathrm{C}$ and $\mathrm{P}_{\mathrm{H} 2}=2$ bars) that deviating within a $2 \%$ range. Clearly, a high degree of kinetic reproducibility has been achieved.

Table 3.1-Comparison of the yield and the properties of polymer obtained by reproducible experiments

\begin{tabular}{lccccccc}
\hline $\begin{array}{c}\text { Run } \\
\text { Y }\end{array}$ & $\begin{array}{c}\mathrm{Y}^{*} \\
(\mathrm{~g})\end{array}$ & $\begin{array}{c}\mathrm{M}_{\mathrm{w}} \\
(\mathrm{kg} / \mathrm{mol})\end{array}$ & $\begin{array}{c}\mathrm{M}_{\mathrm{n}} \\
(\mathrm{kg} / \mathrm{mol})\end{array}$ & $\mathrm{M}_{\mathrm{w}} / \mathrm{M}_{\mathrm{n}}$ & $\begin{array}{c}\mathrm{X}_{\mathrm{C} 1} \\
\%\end{array}$ & $\begin{array}{c}\mathrm{X}_{\mathrm{C} 2} \\
\%\end{array}$ & $\begin{array}{c}\mathrm{Tm} \\
\left({ }^{\circ} \mathrm{C}\right)\end{array}$ \\
\hline 1 & 49.4 & 139.9 & 15.2 & 9.2 & 68.5 & 73.2 & 132.5 \\
2 & 49.7 & 145.4 & 16.1 & 9.03 & 67.6 & 71.6 & 132.3 \\
3 & 49.3 & 142.7 & 15.6 & 9.15 & 68.2 & 72.1 & 132.4 \\
\hline
\end{tabular}

* Y1: Produced polyethylene after an hour of reaction 
The reproducibility of the yield (after one hour of reaction time) was excellent, as shown in Table 3.1. This table also gives an impression of the reproducibility of other polymer properties mentioned above - all of them were measured twice. Only the deviation of the PSD (first run) was a little greater ${ }^{11}$ than for the other two runs, see Figure 3.2.

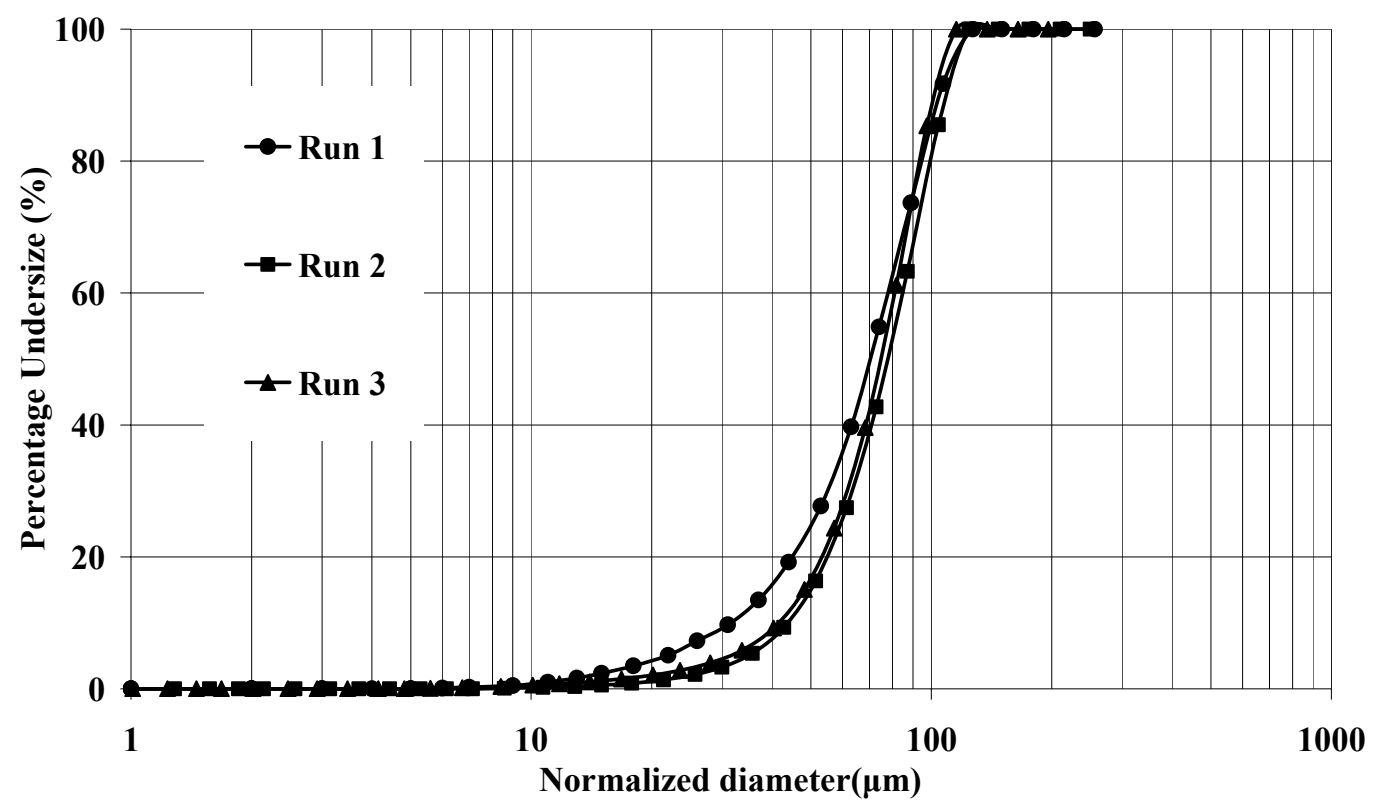

Figure 3.2-Particle size distribution reproducibility of three slurry ethylene polymerizations at $\mathrm{T}=80^{\circ} \mathrm{C}, \mathrm{P}_{\mathrm{C} 2}=2$ bars and $\mathrm{P}_{\mathrm{H} 2}=2$ bars

As a result of above finding, we claim that:

- the reproducibility of experiments is high, the activity of the gas-phase catalyst, $\mathrm{C}_{\mathrm{g}}$, comes close to industrial standards

- all methods selected are precise enough to identify the differences between gas and slurry.

In the following chapter, we will quantify the differences between gas and slurry polymerization.

\subsection{Moving from Gas-Phase to Slurry}

Figure 3.3 shows the influence of the amount of hexane on the reaction rate for three experiments. The reaction rate profiles show different shapes for run-1 (gas-phase experiment using $2 \mathrm{ml} \mathrm{n}$-hexane) and run-2 (slurry experiment using $110 \mathrm{ml} \mathrm{n}$-hexane) compared to run-

\footnotetext{
11 As discussed later intensively, this results from the fact that we came very close to the critical crystallinity for particle disintegration of about $75 \%$.
} 
3 (slurry experiment using $700 \mathrm{ml} \mathrm{n}$-hexane). The time needed to reach the maximum increased with an increasing amount of hexane. The rate-profile for run-2 shows the highest peak.

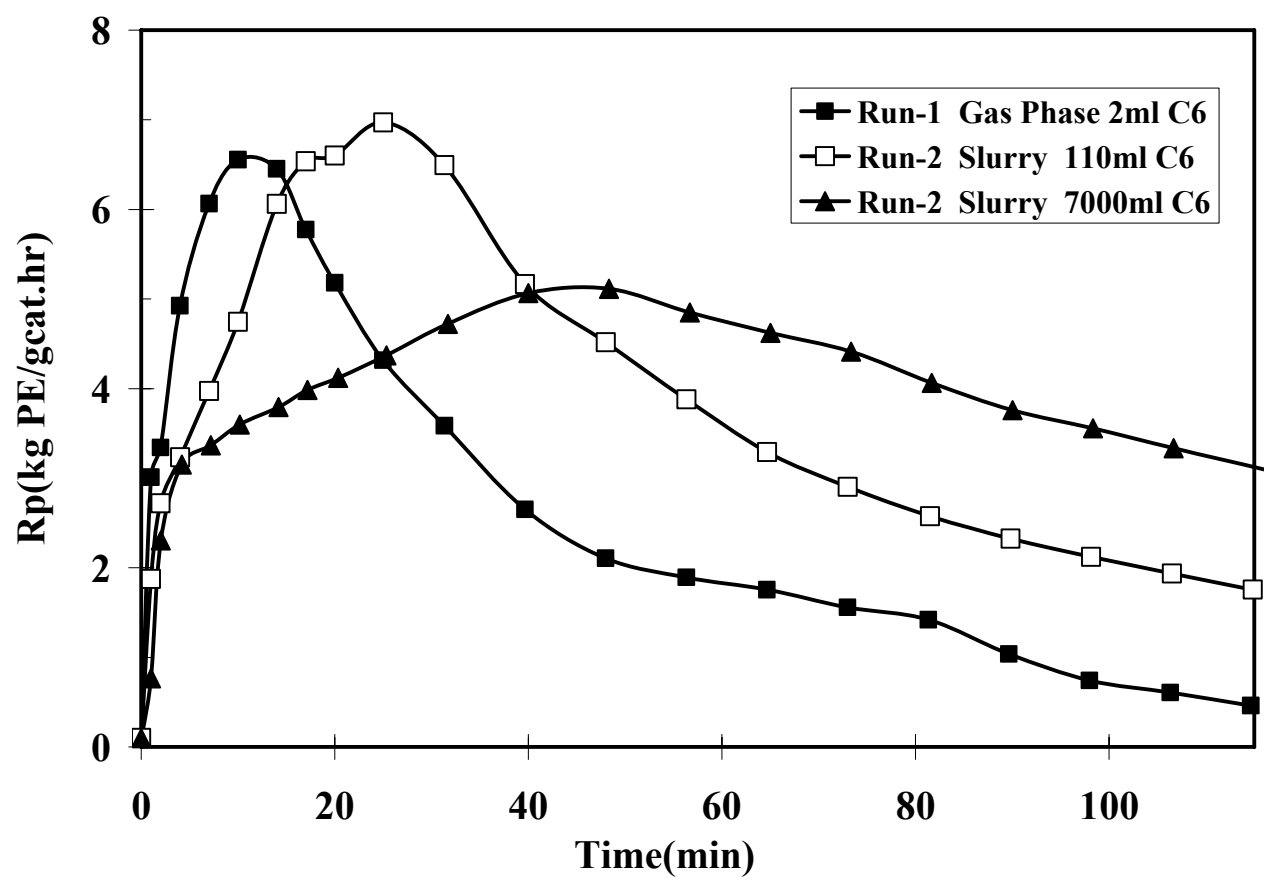

Figure 3.3-The influence of the amount of solvent on homo-ethylene polymerization at $\mathrm{T}=80^{\circ} \mathrm{C}, \mathrm{P}_{\mathrm{C} 2}=\mathbf{2}$ bar and $\mathrm{P}_{\mathrm{H} 2}=\mathbf{2}$ bar

The reason for these very different profiles for the gas-phase and slurry experiment will be fully discussed in the following chapters. For now, we just speculate that the fast activation of the run-1's profile could be partially caused by local overheating of the growing particles in the gas-phase [64], but also different co-catalyst participation in the activation processes might contribute. However, modelling or measuring of the co-catalyst distribution within the particle under permanent fresh polymer generation is hardly possible. Hexane - as a good heat transfer medium - eliminates the local overheating and distributes co-catalyst more homogeneously near the active centres.

For all three experiments, the profile does not differ too much during the first 3 minutes. The catalyst yield after 15 minutes is the highest for the gas-phase reaction. This changes for longer reaction times: after 2 hours, the yield under typical slurry conditions is much higher than in gas-phase polymerization. This is highly significant for process control and commercial application. Since the reaction time in industrial processes is distributed, every particle has an individual residence time in a continuous reactor according to the residence time profile of the given reactor.

Delaying the peak point means working on the safe side in terms of overheating of smaller particles [65], because the maximum heat flow from particle to gas/liquid must then be removed from larger particles. In the case of a smaller amount of the solvent, when the solvent does not form a continuous phase, the role of the solvent changes: wet particles are 
more sticky (which is a disadvantage), but heat removal is more readily achieved by evaporation.

In terms of crystallinity, the $1^{\text {st }}$ and $2^{\text {nd }}$ crystallinity changed in the range of 55 to 71 and 65 to 76 percentage respectively; see Table 3.2. The maximum and minimum crystallinity result from hexane-rich (run-3, $700 \mathrm{ml}$ ) and hexane lean (run-2, $110 \mathrm{ml}$ ) experiments for slurry polymerization, showing a difference between $1^{\text {st }}$ and $2^{\text {nd }}$ crystallinity $\mathrm{X}_{\mathrm{C}(2-1)}$ of $5 \%$ and $10 \%$ respectively. However, the second crystallinity is always higher, such that; $\mathrm{X}_{\mathrm{C}(2-1)}$ is positive. The gas-phase crystallinity lies in the middle of this range, and $\mathrm{X}_{\mathrm{C}(2-}$ ${ }_{1)}=5 \%$. It is well known that low molecular weight PE crystallizes faster. Despite the fact that all MWD produced lie in the same range, one would expect that run-2 would not have resulted the lowest crystallinity. We will retain to this point later, but a first interpretation will be given below.

Table 3.2-Operating conditions and polymer produced properties of solvent series experiments in

\begin{tabular}{|c|c|c|c|c|c|c|c|c|}
\hline Run & $\begin{array}{c}\text { Hexane } \\
(\mathrm{ml})\end{array}$ & $\begin{array}{l}\mathrm{Y}^{*} \\
(\mathrm{~g})\end{array}$ & $\begin{array}{l}\mathrm{Tm} \\
\left({ }^{\circ} \mathrm{C}\right)\end{array}$ & $\begin{array}{c}\mathrm{X}_{\mathrm{C} 1} \\
\%\end{array}$ & $\begin{array}{c}\mathrm{X}_{\mathrm{C} 2} \\
\%\end{array}$ & $\begin{array}{c}\mathrm{M}_{\mathrm{w}} \\
(\mathrm{kg} / \mathrm{mol})\end{array}$ & $\begin{array}{c}\mathrm{M}_{\mathrm{n}} \\
(\mathrm{kg} / \mathrm{mol})\end{array}$ & $\mathrm{M}_{\mathrm{w}} / \mathrm{M}_{\mathrm{n}}$ \\
\hline 1 & 2 & 98 & 131.7 & 67.1 & 72.1 & 143.9 & 13.1 & 11 \\
\hline 2 & 110 & 150 & 132.3 & 55.5 & 65.5 & 138.8 & 12.7 & 10.9 \\
\hline 3 & 700 & 160 & 132.5 & 71.3 & 76.6 & 139.9 & 15.2 & 9.2 \\
\hline
\end{tabular}

Y2: Produced polyethylene after two hours of reaction

For the same bulk reactor temperature, the growing catalyst/polymer particles temperature in the gas-phase is higher compared to slurry. This could lead to a higher chain mobility, resulting in a high crystallinity in run-1. Adding some hexane decreases the particle temperature, but increases the chain mobility by swelling of the amorphous polymer matrix to a certain extent. On the other hand - during equilibrium swelling - hexane can act as a barrier and decrease the crystallization rate. However, this does not explain the low value of the run2 in $\mathrm{X}_{\mathrm{C} 2}$. For the moment, we keep in mind that hexane can increase the chain mobility by swelling the amorphous PE but can decrease the crystallization rate by dilution. The melting temperature does not follow the same trend as the crystallinity, and it is nearly the same for all three experiments.

The MWD, average molecular weights and the polydispersity also lie in the same ranges, but show a higher Mn and lower PD for run-3. Clearly, the chain transfer conditions that lead to dead polymer production are little changed by adding some hexane. Only the experiment with a continuous hexane phase shows a lower Mw tendency combined with formation of a little high-Mw shoulder; see Figure 3.4. 


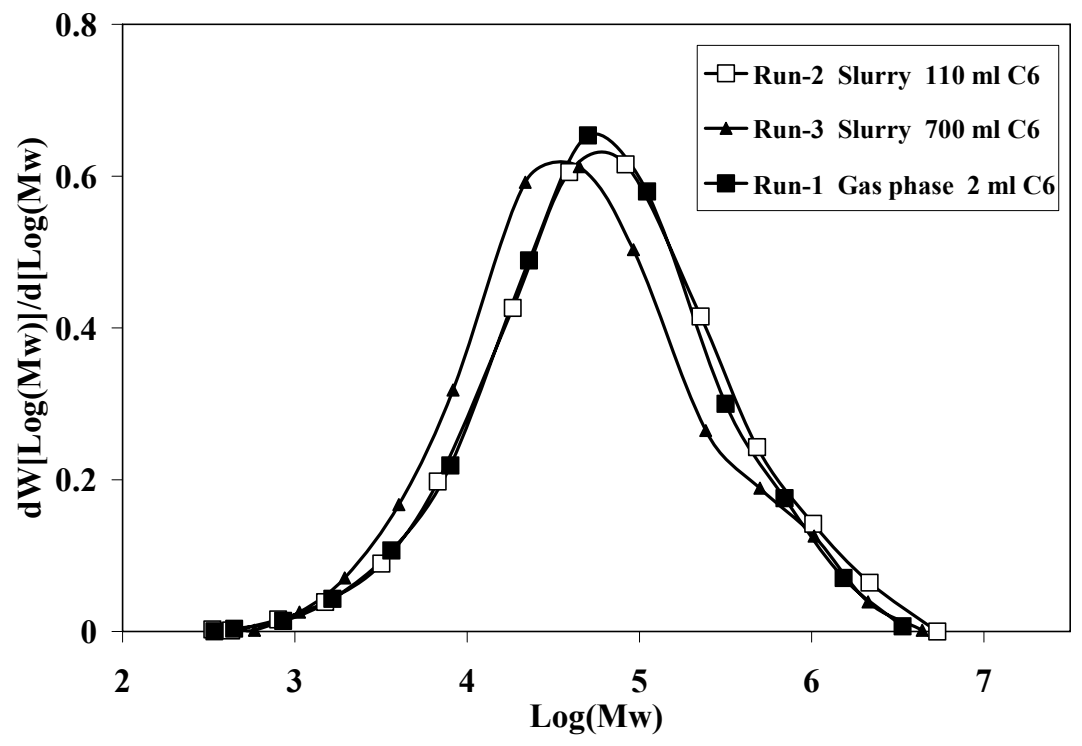

Figure 3.4 -MWDs of the polymer produced in three experiments given in table 3.2

Figure 3.5 shows that changing the amount of n-hexane does not influence the PSD of the polymer produced.

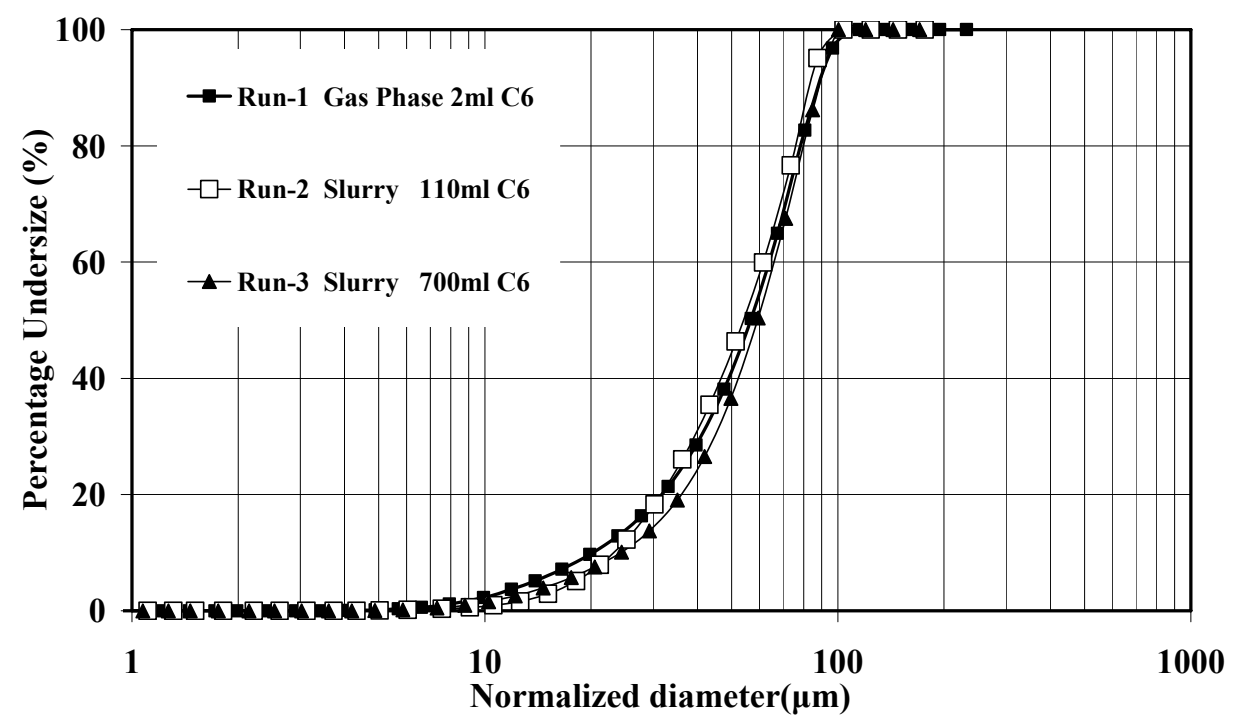

Figure 3.5-Comparison of cumulative PSD profiles normalized with the yield of the polymer extracted from three experiments given in Table 3.2

Finally, under the given range of polymerization conditions, it is concluded that adding n-hexane leads to a remarkable change of the rate profile with low impact on the polymer properties including crystallinity, MWD, melting temperature and PSD.

However, one question remains unanswered: what happens if one combines a very high crystallinity with a high polymerization rate? Such a combination would increase the brittleness of particles and could lead to different results... or not? Actually, in the experiments described above, only moderate values have been reached for both variables. The range of operational conditions should be extended, which will be done in chapter 6 . 


\subsection{Influence of Pre-contacting Time}

As mentioned earlier, the gas-phase catalyst system used in this study needs to be precontacted with TIBA for a given time, before being injected into the reactor. Without precontacting, the yield and productivity of catalyst would be very low.

To investigate the influence of pre-contacting time on the kinetics of polymerization and polymer properties, three slurry experiments with different pre-contacting time were performed. The catalyst $(\mathrm{Cg})$ was firstly weighed in a 3-ml conical vial, and then precontacted with a certain amount of co-catalyst. After that, 2-ml n-hexane was added to the vial and then the mixture was carefully and gently mixed. The vial was kept in the glove box for a given period before being injected into the reactor (pre-contacting time). The precontacting time was varied between 2 minutes and 24 hours. The experiments were run at $80^{\circ} \mathrm{C}, 2$ bar partial pressure of ethylene and 2 bar partial pressure of hydrogen using $700 \mathrm{~mL}$ n-hexane.

Figure 3.6 shows the effect of the pre-contacting time on the polymerization rate profile. One major result was that a minimum contact time was clearly required, but the same profiles result for both 30 minutes and 24 hours. These rate-profiles can be divided into three distinct regions. Reactions start quickly with a high slope in the first region. By increasing the pre-contacting time, a clear increase in the slope of the reaction rate can be observed in the first region. In the second and third regions, the shape of the curve for both 30 minutes and 24 hours pre-contacting start with a relatively higher slope, reaching earlier the peak activity, but also deactivate faster compared to the experiment with 2 minutes contact time. It is worth mentioning that reaction without pre-contacting starts only after a very significant delay following a very low reaction rate.

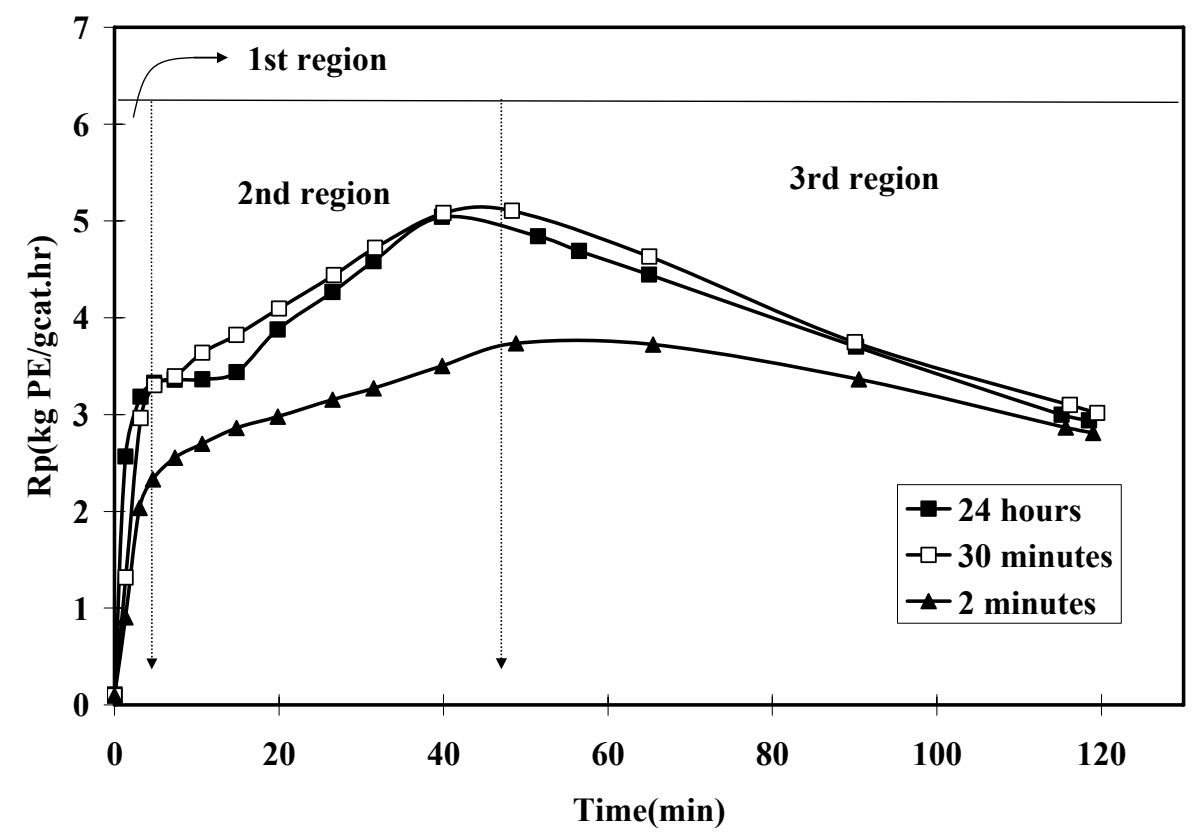

Figure 3.6-Influence of pre-contacting time on reaction rate in slurry ethylene polymerization 
Corresponding to the rate profiles, the MWD curves shown in Figure 3.7 are nearly the same for $30 \mathrm{~min}$ and $24 \mathrm{~h}$ contact times, whereas the curve for $2 \mathrm{~min}$ is significantly broader; see also PD in Table 3.3.

It seems that a catalyst with the lower pre-contacting time has a higher tendency to produce a broader MWD, due to higher heterogeneity of active centres.

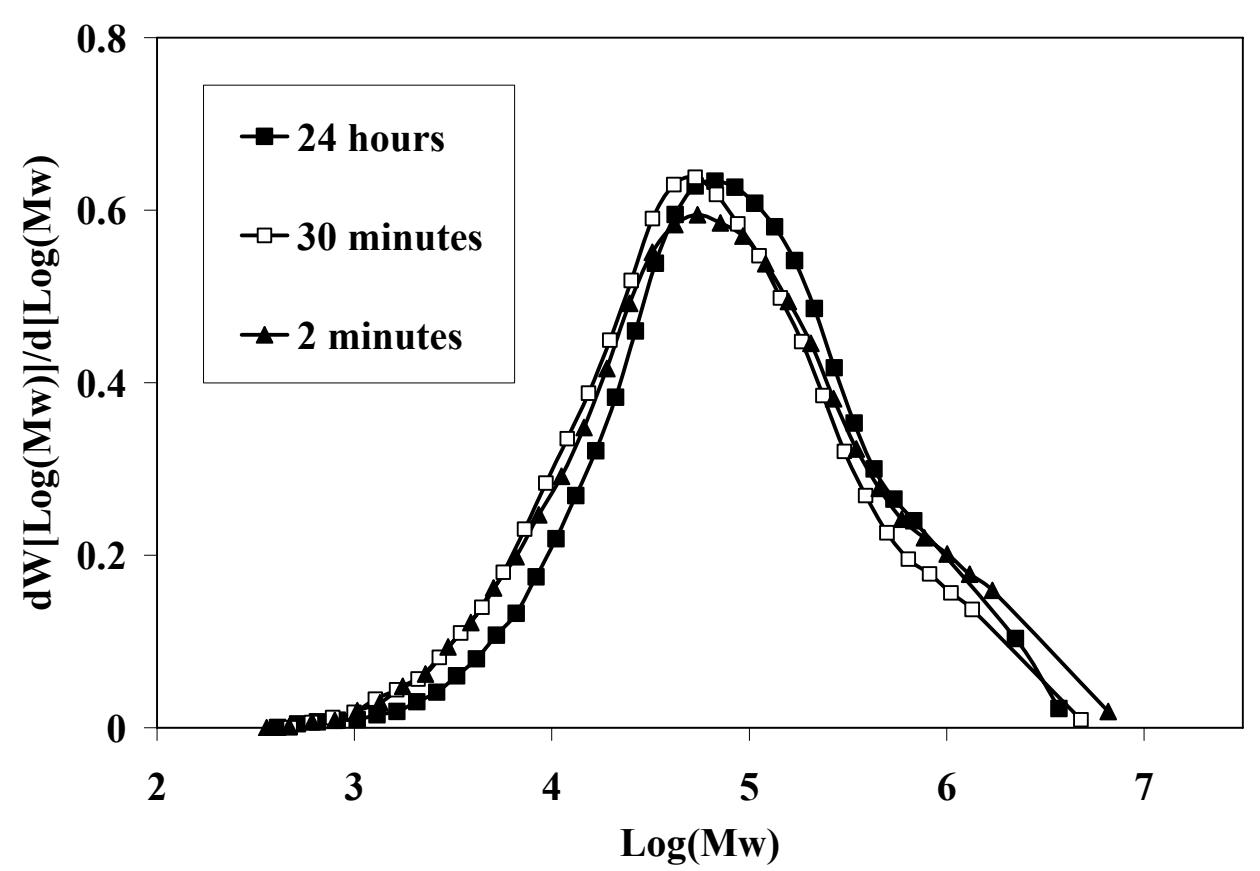

Figure 3.7-The effect of pre-contacting time on molecular weight distribution of polyethylene

$$
\text { powder }
$$

The influence of the contact time on $1^{\text {st }}$ and $2^{\text {nd }}$ crystallinity and melting temperature is not dramatic, but the crystallinity after $30 \mathrm{~min}$ contact is somewhat higher that achieved for 2 min and 24 hours.

Table 3.3-Comparison of yield and the properties of polymer obtained by pre-contacting time

\begin{tabular}{lccccccc}
\multicolumn{1}{c}{ Run } & $\begin{array}{c}\text { Yield } \\
(\mathrm{g})\end{array}$ & $\begin{array}{c}\mathrm{M}_{\mathrm{w}} \\
\mathrm{kg} / \mathrm{mol})\end{array}$ & $\begin{array}{c}\mathrm{M}_{\mathrm{n}} \\
\mathrm{kg} / \mathrm{mol})\end{array}$ & $\mathrm{M}_{\mathrm{w}} / \mathrm{M}_{\mathrm{n}}$ & $\begin{array}{c}\mathrm{X}_{\mathrm{C} 1} \\
\%\end{array}$ & $\begin{array}{c}\mathrm{X}_{\mathrm{C} 2} \\
\%\end{array}$ & $\begin{array}{c}\mathrm{Tm} \\
\left({ }^{\circ} \mathrm{C}\right)\end{array}$ \\
\hline 24 hours & 153 & 160.8 & 17.9 & 9 & 70.4 & 74.1 & 133.3 \\
30 minutes & 158 & 151.5 & 15.5 & 9.8 & 75.1 & 79.4 & 131.6 \\
2 minutes & 116 & 182.3 & 15.2 & 12 & 67.8 & 73 & 132.5 \\
\hline
\end{tabular}

Y2: Produced polyethylene after two hours of reaction

For the 2 minutes and 30 minutes of pre-contacting, the PSDs almost overlap each other; see fig. 3.8. However, in the case of pre-contacting for 24 hours, the particle size distribution curve shifts significantly to the left towards low particle size. Many fines are generated after such extremely long contact times. 


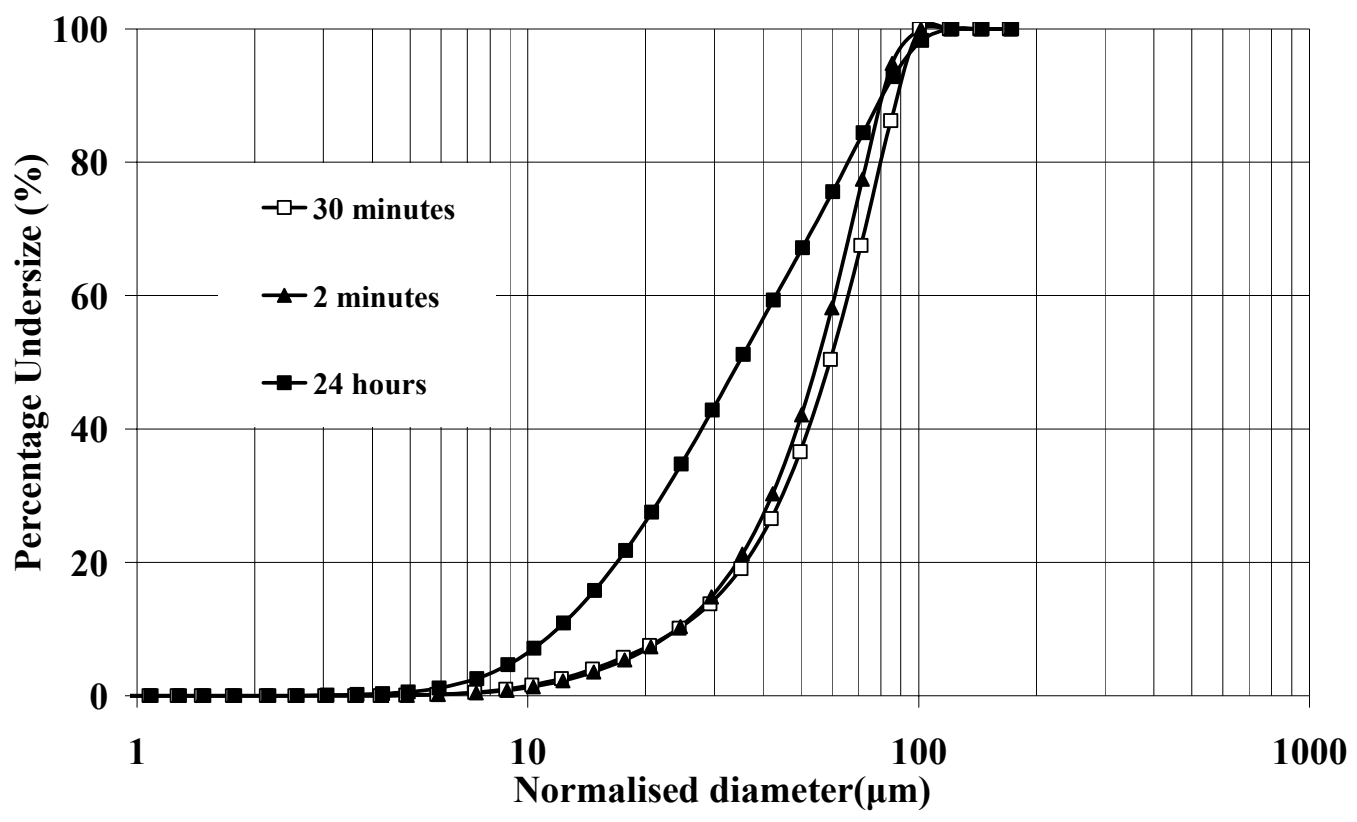

Figure 3.8-The effect of pre-contacting on particle size distribution of polyethylene powder

We realize that, under these conditions, fines generation does NOT lead to a dramatic change in the polymerization rate curve ... with one exception: the slope during the "first minute" is much higher after $24 \mathrm{~h}$ contact time; see Figure 3.6. It seems that early stage conditions play a huge role [27].

An initial interpretation:

Since co-catalyst has more time to diffuse into the pore of the catalyst during 24 hours, more centers are potentially susceptible to being active and therefore start polymerization. These more potentially active centres produce more heat in the early stage of polymerization, leading to increases in both thermal and growth stresses [66] inside the growing catalyst particle. More thermal and growth stress inside the growing particle lead to more fines production. This allows a faster start. However, fragmentation occurs also in the 30-minutes precontacting experiment, and this experiment will reach the same level of activation after a short period of time.

\subsection{Influence of Reaction Time}

Figure 3.9 shows the reaction rate-profiles of three slurry experiments executed at $\mathrm{T}=$ $90^{\circ} \mathrm{C}, \mathrm{P}_{\mathrm{H} 2}=2$ bar and $\mathrm{P}_{\mathrm{C} 2}=2$ bar in hexane. The $\mathrm{Cg}$ catalyst system was used. The three reactions were stopped at 45, 100 and 120 minutes. All three profiles start with very fast activation followed by a long-lasting period of time with fairly constant polymerization rates at the same plateau. 


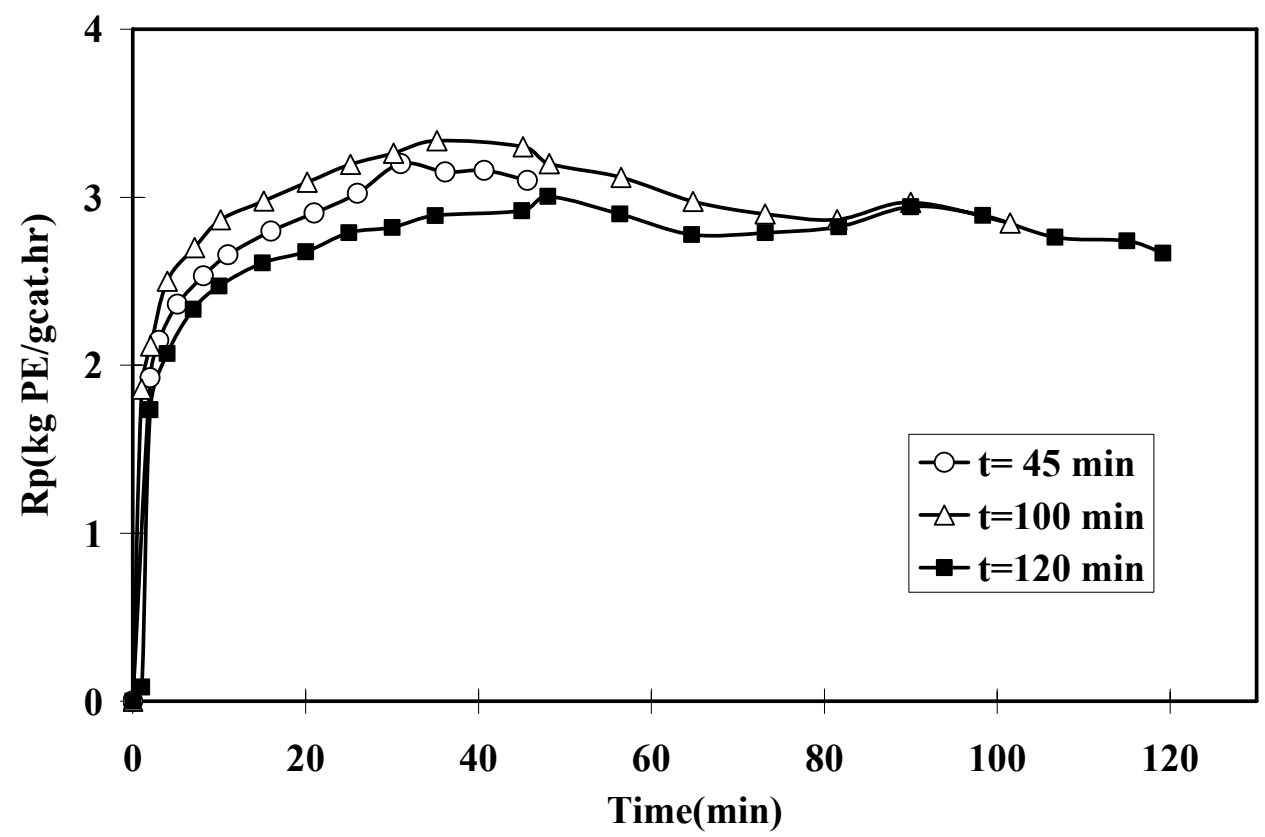

Figure 3.9-The reaction rate-profile for time series slurry experiments at $\mathrm{T}=90^{\circ} \mathrm{C}, \mathrm{P}_{\mathrm{H} 2}=2$ bar and

$$
\mathbf{P}_{\mathrm{C} 2}=2 \text { bar }
$$

With increasing reaction time, a noticeable increase in $M_{w}$ and $M_{n}$ is observed accompanied by a small increase in the PD; see Table 3.4. The multi-site nature of ZN catalysts is well known; see Cecchin-1983, Floyd-1988, [53]. Kissin in 1999 and Hakim in 2008 [46, 67] also found an increase in molecular weight as reaction time increased. By deconvolution analysis, Kissin showed that those centres producing low molecular weight polymer deactivate faster than those producing high molecular weight. This is fully supported by our results.

The crystallinity follows the same pattern as the molecular weight - lower Mw leads to higher crystallinity.

Table 3.4 -Operating conditions and polymer produced properties of three mentioned

\begin{tabular}{cccccccccc}
\multicolumn{10}{c}{ experiments in slurry ethylene polymerization at $\mathbf{T}=\mathbf{9 0}^{\circ} \mathbf{C}$} \\
\hline Run & $\mathrm{P}_{\mathrm{C} 2}$ & $\mathrm{P}_{\mathrm{H} 2}$ & $\mathrm{Time}$ & $\mathrm{Tm}$ & $\mathrm{X}_{\mathrm{C} 1}$ & $\mathrm{X}_{\mathrm{C} 2}$ & $\begin{array}{c}\mathrm{M}_{\mathrm{w}} \\
(\mathrm{kg} / \mathrm{mol})\end{array}$ & $\begin{array}{c}\mathrm{M}_{\mathrm{n}} \\
(\mathrm{kg} / \mathrm{mol})\end{array}$ & $\mathrm{M}_{\mathrm{w}} / \mathrm{M}_{\mathrm{n}}$ \\
& $(\mathrm{bar})$ & $($ bar $)$ & $(\mathrm{min})$ & $\left({ }^{\circ} \mathrm{C}\right)$ & $\%$ & $\%$ & 13 & \\
\hline Run1 & 2 & 2 & 121 & 132.1 & 72 & 77 & 137 & 13.4 & 10.2 \\
Run2 & 2 & 2 & 101 & 131.8 & 73 & 77 & 115 & 11.7 & 9.8 \\
Run3 & 2 & 2 & 45 & 131.2 & 77 & 82 & 100 & 11. & 9.1 \\
\hline
\end{tabular}

The MWD slightly shifts towards higher molecular weight with lengthening times. It is interesting that a distinct shoulder is always formed in the high-Mw region. 


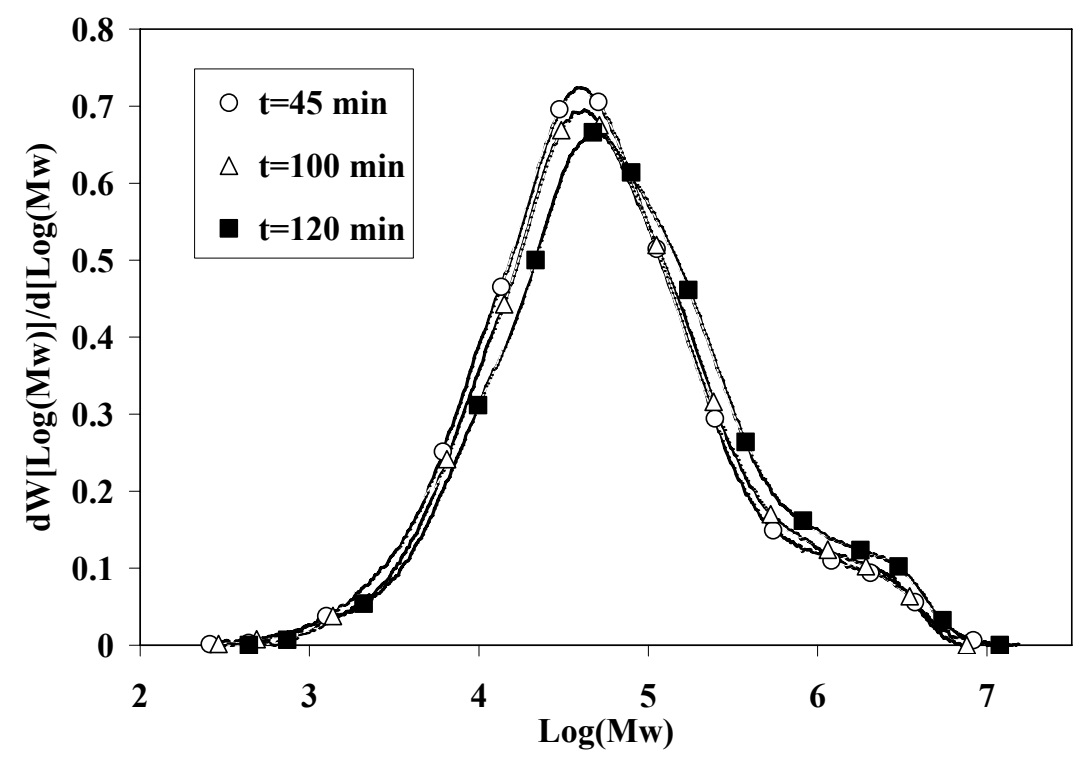

Figure 3.10-MWDs of the polymer produced in three experiments given in Table 3.4

For the gas-phase catalyst in slurry ethylene polymerization, one can conclude that reaction time (between 45 to 120 minutes) has little effect on the particle size distribution. Figure 3.11 shows the normalized PSD profiles measured by a LDPSA. The deviation of the 100min experiment (more fines) is assumed to be within the reproducibility limit under these conditions, and this deviation also explains the higher polymerization rate; see Figure 3.9.

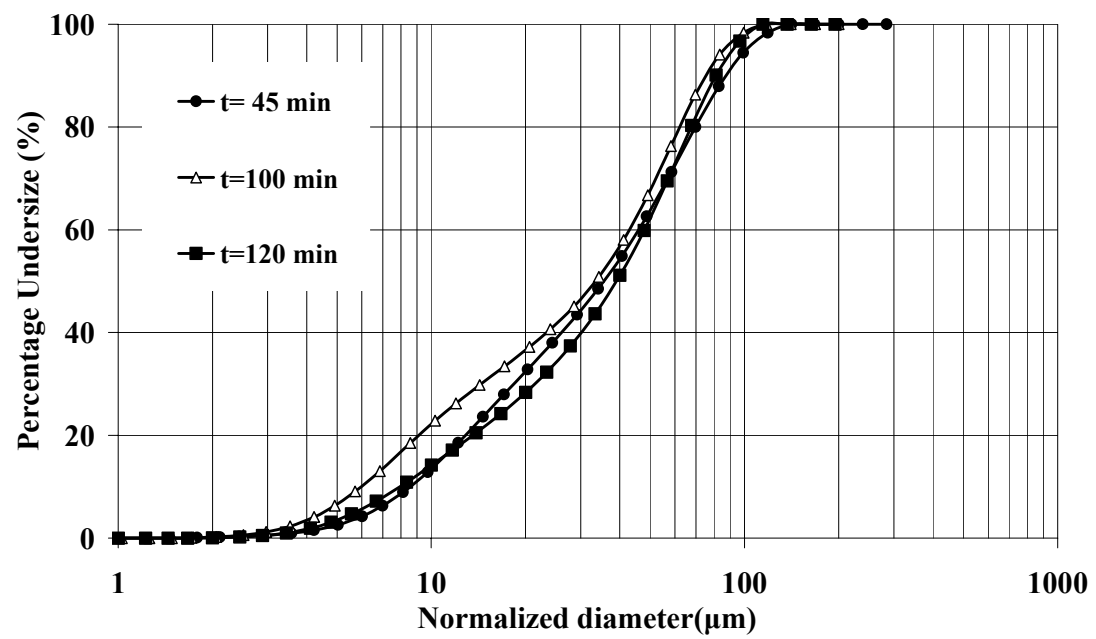

Figure 3.11-Comparison of cumulative PSD profiles normalized with the yield of the polymer extracted from the three experiments listed in Table 3.4

The results presented in Chapter 3.5 give a good reason to start a new series of basic experiments to clarify the influence of reaction time. Again, this is a most important commercial aspect, because every particle has its own individual residence time in a continuous (industrial) reactor. If the multi-site $\mathrm{ZN}$ catalyst activates and deactivates different sites in different ways, then the polymer produced in every single particle changes its properties over the individual residence time. This adds another difficulty to scaling-up from batch to continuous processes. Therefore, some further experiments under different operating 
conditions were performed. Table 3.5 gives an overview of the experimental conditions and results.

With reaction time

- The $1^{\text {st }}$ and $2^{\text {nd }}$ crystallinity decreased. This decreasing is more pronounced in the presence of hydrogen, pair A and B. Lower $\mathrm{Mw}$ (higher $\mathrm{H}_{2}$ ) corresponds to higher crystallinity. Note that in experiment A, nearly $75 \%$ crystallinity is reached in-situ.

- $\mathrm{M}_{\mathrm{w}}$ of the longer-lasting experiments increased.

- $M_{n}$, in the case of high hydrogen (6 bar) and without hydrogen decreased, but is nearly constant in the case of two bar $\mathrm{H}_{2}$.

- PD as a consequence of the Mw and Mn behaviour increased.

Naturally, this behaviour can also be found in the MWD; see Figure 3.12.

Table 3.5-Operating conditions and properties of polymer produced of six experiments in slurry

\begin{tabular}{cccccccccc}
\multicolumn{10}{c}{ ethylene polymerization } \\
\hline Run & $\mathrm{P}_{\mathrm{C} 2}$ & $\mathrm{P}_{\mathrm{H} 2}$ & Time & $\mathrm{Tm}$ & $\mathrm{X}_{\mathrm{C} 1}$ & $\mathrm{X}_{\mathrm{C} 2}$ & $\mathrm{M}_{\mathrm{w}}$ & $\mathrm{M}_{\mathrm{n}}$ & $\mathrm{M}_{\mathrm{w}} / \mathrm{M}_{\mathrm{n}}$ \\
& $($ bar $)$ & $($ bar $)$ & $(\mathrm{min})$ & $\left({ }^{\circ} \mathrm{C}\right)$ & $\%$ & $\%$ & $(\mathrm{~kg} / \mathrm{mol})$ & $(\mathrm{kg} / \mathrm{mol})$ & \\
\hline $\mathrm{A} 1$ & 2 & 6 & 12 & 131.3 & 75 & 85.4 & 46.3 & 6.9 & 6.7 \\
$\mathrm{~A} 2$ & 2 & 6 & 60 & 129.3 & 74.7 & 80.3 & 71.3 & 6.2 & 11.4 \\
$\mathrm{~B} 1$ & 2 & 2 & 11 & 131.6 & 73.5 & 84.6 & 105.6 & 11.9 & 8.8 \\
$\mathrm{~B} 2$ & 2 & 2 & 60 & 132.5 & 68.5 & 73.9 & 156.7 & 12.3 & 12.7 \\
$\mathrm{C} 1$ & 2 & 0 & 36.5 & 139.2 & 64.6 & 57.3 & 626.2 & 151.9 & 4.1 \\
$\mathrm{C} 2$ & 2 & 0 & 54 & 136.4 & 64.1 & 56.4 & 847.7 & 121 & 7.0 \\
\hline
\end{tabular}

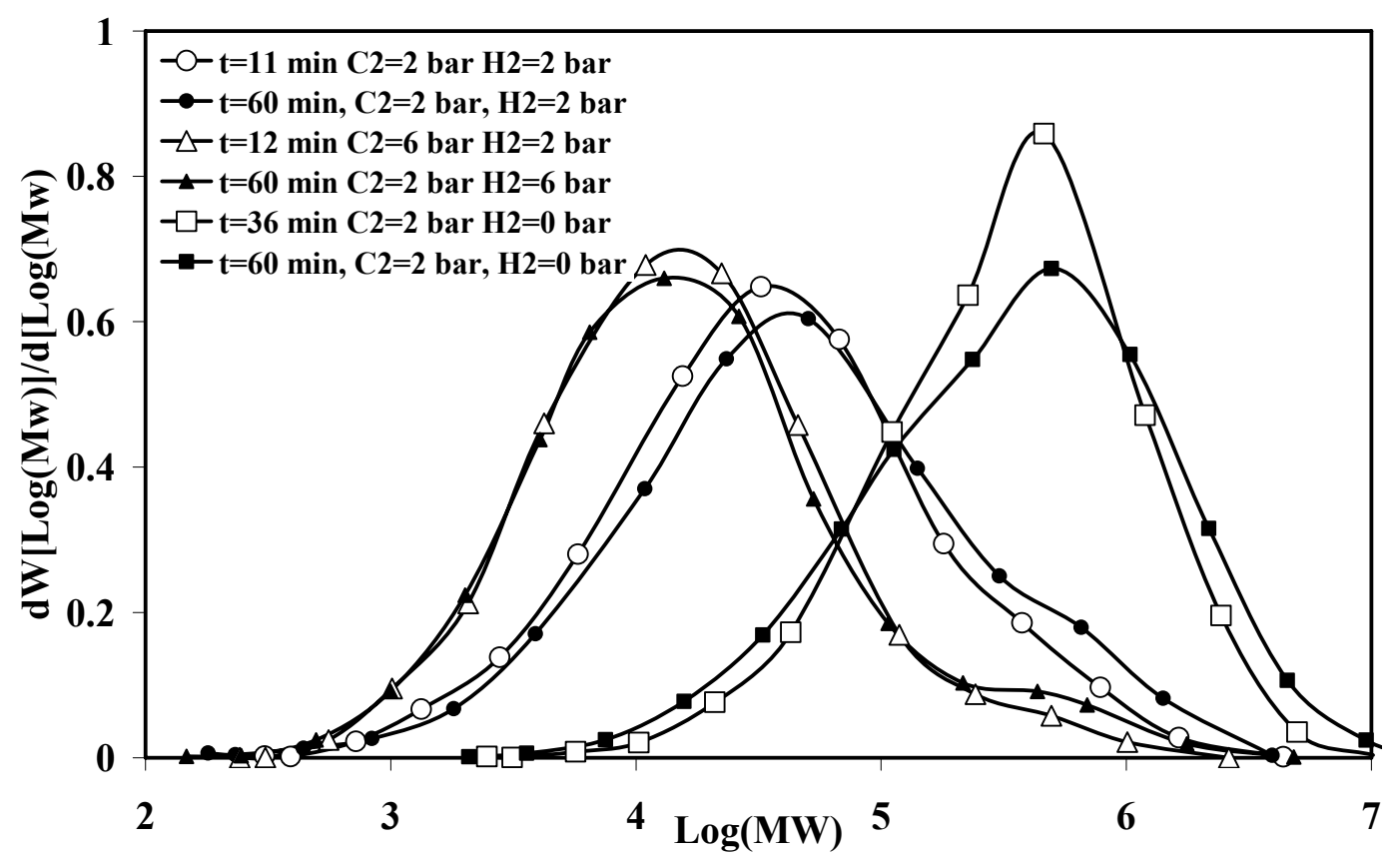

Figure 3.12-MWDs of the polymer produced in six experiments given in Table 3.5 


\subsection{Nitrogen Influence}

Weickert et al, in 1999 [25] investigated the influence of the concentration of inert materials on the rate of polymerization for the first time. They found that when the concentration of inert material was increased, the reaction rate decreased. The authors explained this in terms of the inert gas enrichment effect inside the particles.

Pinto et al, in 2005 [68] proposed a two-phase dynamic model to describe the early stage of polymerization inside the catalyst particles. They found that the concentration of inert material in catalyst particles could influence particle fragmentation during the early stage of polymerization. The authors hypothesized that the inert materials could reduce the concentration of monomer around the active site, and this could lead to a decreasing reaction rate during the early stage of polymerization with a direct impact on particle fragmentation. Thus, higher inert concentration can lead to more moderate fragmentation, in turn resulting in a more uniform particle morphology.

Series of gas-phase ethylene polymerization experiments were conducted by varying the nitrogen partial pressure while keeping all other parameters constant. The common conditions at the start of each reaction were as follows: catalyst $(20 \mathrm{mg})$ was firstly weighed in a $3-\mathrm{ml}$ conical vial, and then pre-contacted by a given amount of co-catalyst $(200 \mathrm{mg})$. Next, 2-ml n-hexane was added to the vial and then the mixture was carefully and gently mixed. The vial was kept in the glove box for a given period before being injected to the reactor (pre-contacting time $\approx 30$ minutes). The ethylene partial pressure was 2 bar, the polymerization temperature was $60{ }^{\circ} \mathrm{C} .110 \mathrm{~g}$ salt $(\mathrm{NaCl}$ powder) used as a bed, mixed with $200 \mathrm{mg}$ TIBA as a scavenger for 15 minutes at reaction temperature. The experiments were carried out under isothermal and isobaric conditions as described in Chapter 2.

Figure 3.13 shows the reaction rate profiles for four nitrogen series experiments performed in the gas-phase.

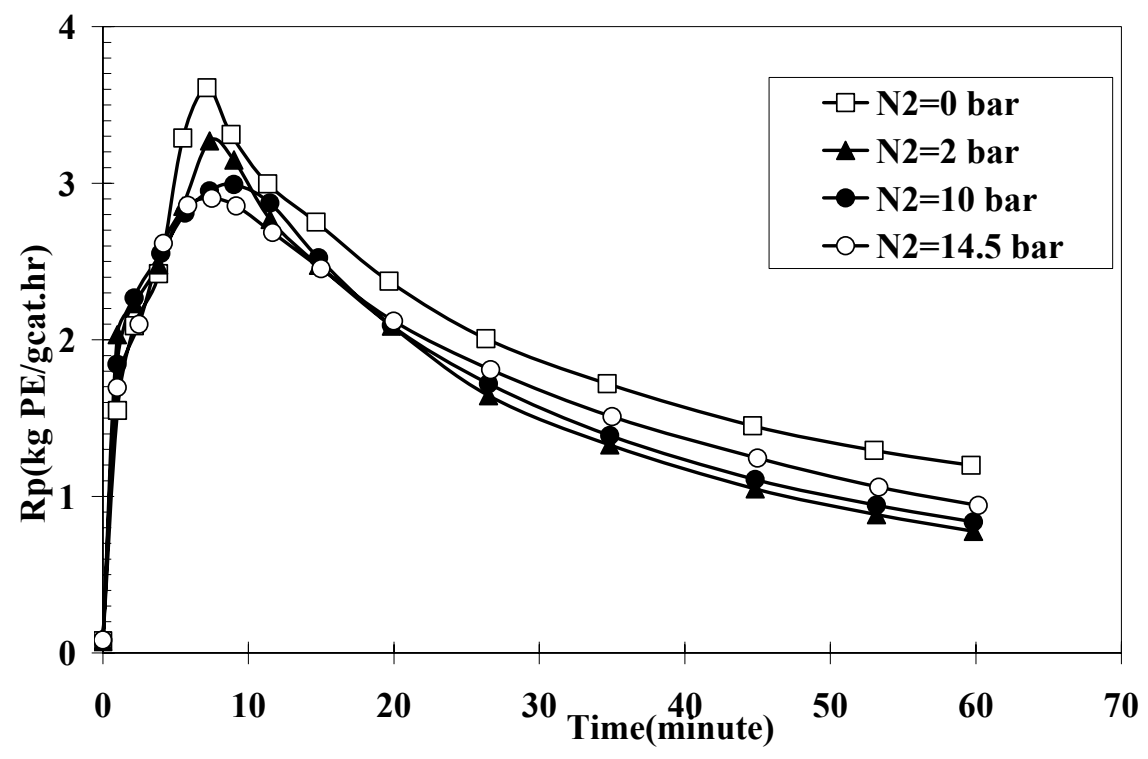

Figure 3.13-The reaction rate-profile for time series slurry experiments at $\mathrm{T}=60^{\circ} \mathrm{C}, \mathrm{P}_{\mathrm{H} 2}=\mathbf{2} \mathrm{bar}$ and $\mathbf{P}_{\mathrm{C} 2}=\mathbf{2}$ bar 
Except for the experiment performed without nitrogen, no significant difference can be observed in terms of changes in activation, deactivation behaviour or yield of experiments as nitrogen partial pressure increased - even with zero nitrogen, the deviation is small enough to be neglected in our further studies. Kissin in 1989 [18] also performed series of experiment with ethylene-nitrogen mixtures. He found that only ethylene partial pressure affects the polymerization rate.

As can be seen from Table 3.6, there is no significant increase or decrease to $M_{w}$, $\mathrm{M}_{\mathrm{n}}, \mathrm{M}_{\mathrm{w}} / \mathrm{M}_{\mathrm{n}}$, 1st crystallinity, 2nd crystallinity and the melting temperature of produced polymer as the nitrogen partial pressure is increased from 0 to 14.5 bar. This is in agreement with the MWD, as shown in Figure 3.14. Increasing nitrogen pressure has no significant influence on particle size and the particle size distribution of samples, with the exception on $\mathrm{P}_{\mathrm{N} 2}=14.5$ bar, see Figure 3.15 .

Clearly, $\mathrm{C}_{\mathrm{g}}$ catalyst does not suffer from transport limitations. In particular, inert enrichment is absent: the pore size, porosity and particle size are all such that these effects cannot be observed.

Table 3.6-Comparison of the properties of polymer obtained in nitrogen series

\begin{tabular}{cccccccc}
\hline Run & $\begin{array}{c}\mathrm{P}_{\mathrm{N} 2} \\
(\text { bar })\end{array}$ & $\begin{array}{c}\mathrm{M}_{\mathrm{w}} \\
(\mathrm{kg} / \mathrm{mol})\end{array}$ & $\begin{array}{c}\mathrm{M}_{\mathrm{n}} \\
(\mathrm{kg} / \mathrm{mol})\end{array}$ & $\mathrm{M}_{\mathrm{w}} / \mathrm{M}_{\mathrm{n}}$ & $\begin{array}{c}\mathrm{X}_{\mathrm{C} 1} \\
\%\end{array}$ & $\begin{array}{c}\mathrm{X}_{\mathrm{C} 2} \\
\%\end{array}$ & $\begin{array}{c}\mathrm{Tm} \\
\left({ }^{\circ} \mathrm{C}\right)\end{array}$ \\
\hline 1 & 0 & 189 & 25.6 & 7.4 & 64.5 & 67.1 & 135.6 \\
2 & 2 & 208 & 26.5 & 7.8 & 63.6 & 67.1 & 135.6 \\
3 & 10 & 202 & 24.5 & 8.2 & 60.6 & 63.7 & 135 \\
4 & 14.5 & 205 & 25.5 & 8 & 64.5 & 68.8 & 133.8 \\
\hline
\end{tabular}

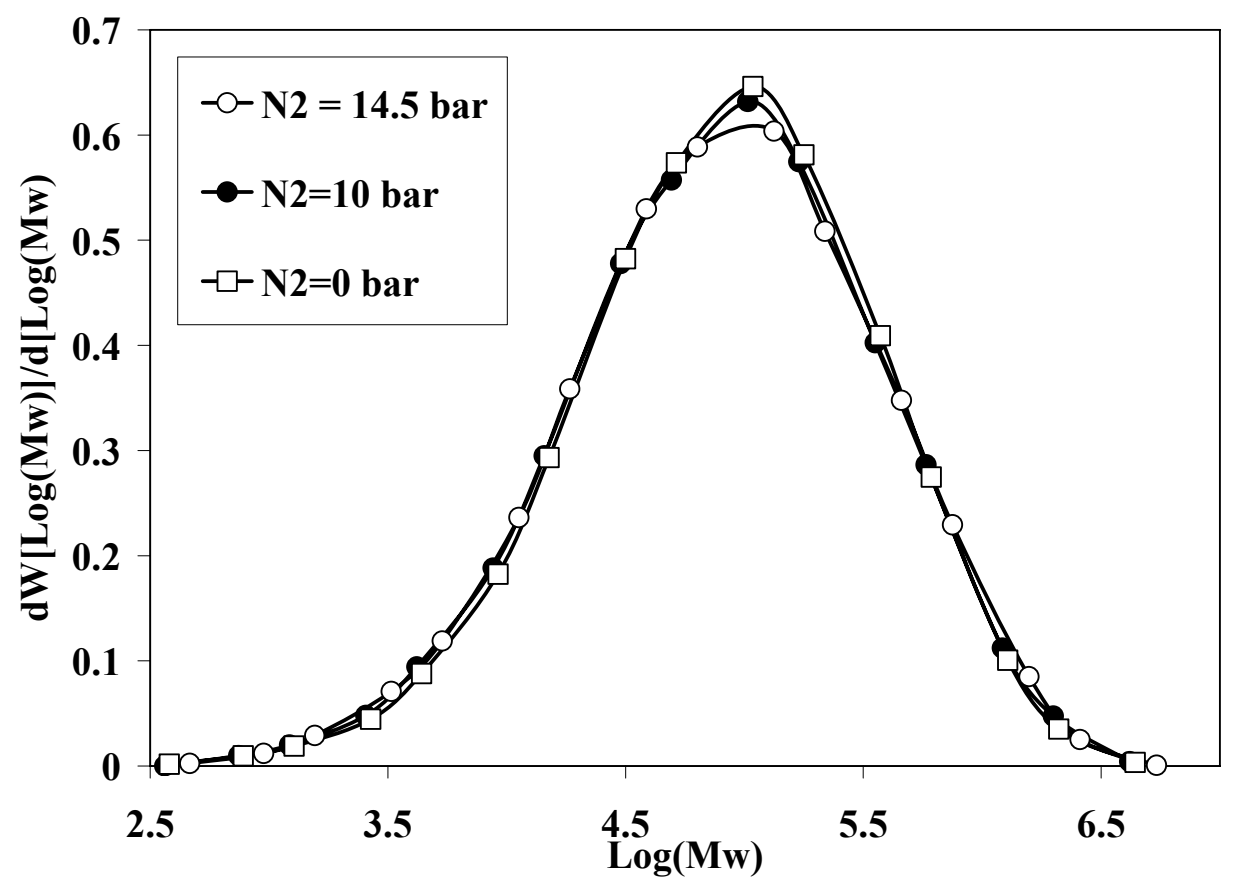

Figure 3.14-MWDs of the polymer produced in nitrogen series experiments given in Table 3.6 


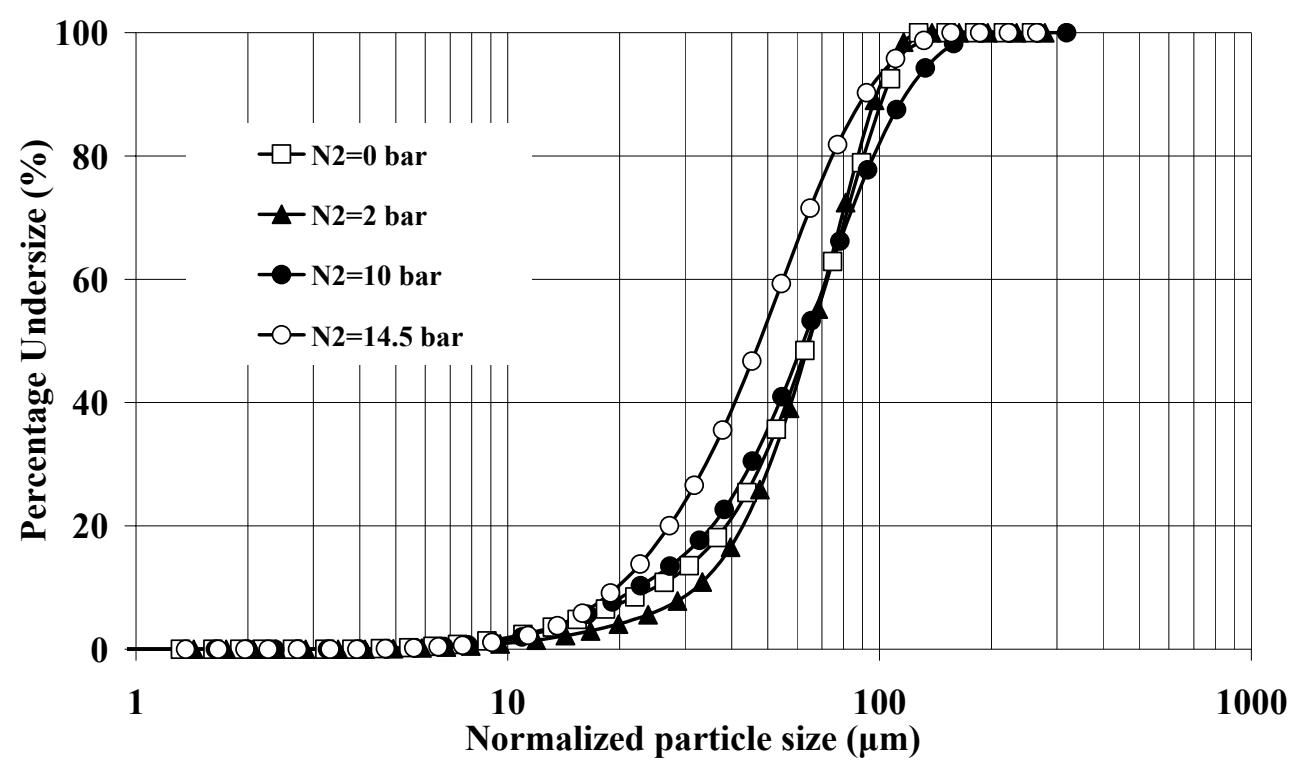

Figure 3.15-Comparison of cumulative PSD profiles normalized with the yield of the polymer extracted from nitrogen series experiments

\subsection{Conclusions}

In this chapter, by performing five series of experiments, the results listed below have been achieved.

Good reproducibility was achieved in terms of kinetics and polymer characterization for all methods selected.

Changing the amount of solvent can dramatically change the reaction rate profiles; hexane is not at all "inert" - it changes all relevant transport and equilibrium conditions.

The catalyst needs to be pre-contacted for a certain time before being injected into the polymerization reactor. Short pre-contacting time leads to low yield; in contrast, long pre-contacting time results in fines formation.

The molecular weight changes with reaction time; this can be attributed to the multi-site nature of $\mathrm{ZN}$ catalysts. The highest crystallinity was obtained during the initial phase.

It was found that the partial pressure of nitrogen has no significant impact on the rate of reaction or on the properties of the produced polymer. 



\section{Chapter 4}

\section{The Influence of Temperature}

\subsection{Introduction}

The polymerization temperature is one of the most important parameters in all polyolefin processes. Increasing the temperature can increase or decrease both the polymerization rate and yield, and usually changes all the polymer properties discussed in this work: molecular weight, crystallinity, and particle morphology. Particle agglomeration, fines generation, wall sheeting and lump formation depend on effective control of reaction temperature.

At the start of polymerization, the surface of the catalyst particles, which include hundreds of millions of active centers, are 'attacked' by monomers. Polymerization starts by forming a primary layer of polymer around active centers. This primary layer increases the internal tension inside the pores of the catalyst, leading to fragmentation of the structure and generation of new active sites. This early stage of polymerization is essential for final product properties. Heat transfer from growing particle in this early stage is also an important aspect of polymerization [65]. During this stage, the external surface of growing particles, which is needed for heat release produced by polymerization, is minimal as long as the particle does not disintegrate. If the heat transfer from particles to surrounding medium is limited, the temperature of the growing particles will increase rapidly [47]. Therefore, softening and - in extreme case - melting of the growing particles is more critical for gas-phase polymerizations. Some authors have reported (using IR thermography) that the surface temperature of such particles could be up to $30^{\circ} \mathrm{C}$ higher than the bulk temperature [26]. Keeping the bulk temperature of the reactor constant and the particle temperature below the softening temperature of the polymer is the best arrangement for a catalytic polyolefin polymerization reactor [69].

A good example to give a better understanding of the gas-phase polymerization phenomena was given by W.H. Ray et al [22]. The authors studied the effects of co-monomer composition, temperature, hydrogen concentration, and $\mathrm{Al} / \mathrm{Ti}$ ratio on kinetics of ethylene/propylene (homo/co) gas-phase polymerization using a $\mathrm{TiCl} 4 / \mathrm{MgCl} 2$ catalyst. Increasing the reaction temperature from $50^{\circ} \mathrm{C}$ to $99^{\circ} \mathrm{C}$ in ethylene homo-polymerization in the presence of $5 \%$ hydrogen, they found an increasing initial polymerization rate, but the polymerization rate then decreased at higher bulk temperatures. SEM and mercury porosimetry measurements revealed an increase in mass transfer resistance of monomer at high temperature, due to softening and partial melting. 
Due to the much higher heat transfer rate particle-bulk, the overheating phenomena discussed above do not exist in slurry polymerizations - at least not under common industrial conditions. Different sorption, diffusion, swelling, and particle morphology development around the active sites in slurry and gas-phase change both the kinetics and polymer properties. Despite the fact of this huge difference between the particle heat balance in gasphase and slurry polymerizations, nothing in the literature directly compares the temperature influence on polymerization kinetics in these two most important industrial processes, taking into account the development of molecular weight, crystallinity and particle size distribution of the polymer produced.

However, it should be possible to describe both processes using the same model - if one takes into account all relevant changes on the micro-, meso- and macro-levels. This chapter will describe the development of such a model ${ }^{1}$.

The first step towards this goal must be the experimentally corroborated quantification of the differences between the slurry and gas-phase. Therefore, the investigation described in this chapter was carried out to compare, identify and evaluate precisely the influence of temperature on slurry and gas-phase ethylene homo-polymerization, by measuring:

- polymerization rate

- molecular weight

- crystallinity

- particle size distribution

with the help of scanning electron microscopy (SEM) and transmission electron microscopy (TEM).

Reactor, chemicals and their purification, and basic procedures were described in Chapter 2.

By varying the reaction temperature, three series of ethylene polymerization experiments were performed:

- $\quad 1^{\text {st }}$ series: in slurry phase in the absence of hydrogen

- $\quad 2^{\text {nd }}$ series: in slurry phase in the presence of hydrogen $\left(\mathrm{P}_{\mathrm{H} 2}=2\right.$ bar $)$

- $\quad 3^{\text {rd }}$ series: in gas-phase in the presence of hydrogen $\left(\mathrm{P}_{\mathrm{H} 2}=2\right.$ bar $)$.

The common conditions at the start of reaction were as follows: catalyst $(20 \mathrm{mg})$ was first weighed in a 3-ml conical vial and then pre-contacted with 200mg of TIBA. 2-ml nhexane was mixed with the vial content. The vial was kept in the glove box for 30 minutes before being injected to the reactor (pre-contacting time of 30 minutes).

Ethylene and hydrogen partial pressures were initially set to 2 bar. For slurry experiments, $700 \mathrm{ml} \mathrm{n}$-hexane was used as solvent mixed with $200 \mathrm{mg}$ TIBA for 15 minutes at reaction temperature. For gas-phase experiments, $110 \mathrm{~g}$ salt ( $\mathrm{NaCl}$ powder) was used as a bed, mixed with $200 \mathrm{mg}$ TIBA for 15 minutes at reaction temperature.

\footnotetext{
${ }^{1}$ please do not read "model" as "mathematical model"
} 


\subsection{Slurry polymerization in the absence of hydrogen}

Selected data of the $1^{\text {st }}$ series of experiments are given in Table 4.1:

Table 4.1-Operating conditions and polymer produced properties of the $1^{\text {st }}$ series

\begin{tabular}{ccccccccc}
\hline Run & $\begin{array}{c}\mathrm{T} \\
\left({ }^{\circ} \mathrm{C}\right)\end{array}$ & $\begin{array}{c}\mathrm{Cc}_{2} \\
\mathrm{~g} / \mathrm{L}\end{array}$ & $\begin{array}{c}R_{p a} \\
(\mathrm{~kg} \mathrm{PE} / \mathrm{gcat} . \mathrm{hr})\end{array}$ & $\begin{array}{c}\mathrm{Xc}_{1} \\
\%\end{array}$ & $\begin{array}{c}\mathrm{Xc}_{2} \\
\%\end{array}$ & $\begin{array}{c}\mathrm{M}_{\mathrm{w}} \\
(\mathrm{kg} / \mathrm{mol})\end{array}$ & $\begin{array}{c}\mathrm{M}_{\mathrm{n}} \\
(\mathrm{kg} / \mathrm{mol})\end{array}$ & $\begin{array}{c}\mathrm{PD}^{1}= \\
\mathrm{M}_{\mathrm{w}} / \mathrm{M}_{\mathrm{n}}\end{array}$ \\
\hline 1 & 50 & 6.9 & 1.0 & 59.4 & 47.7 & 1038 & 289 & 3.6 \\
2 & 70 & 5.4 & 1.8 & 64.1 & 58.7 & 1030 & 282 & 3.7 \\
3 & 80 & 4.7 & 2.5 & 66.9 & 60.4 & 626 & 152 & 4.1 \\
4 & 90 & 4.2 & 4 & 66.1 & 60.4 & 683 & 190 & 3.6 \\
\hline
\end{tabular}

$R_{p a}$ is expressed as (kg PE/gcat.hr)

The ethylene bulk concentration, which was calculated by Aspen Polymer Plus software, decreased from $6.89 \mathrm{~g} / \mathrm{L}$ to $4.23 \mathrm{~g} / \mathrm{L}$ as the temperature increased from $50{ }^{\circ} \mathrm{C}$ to $90^{\circ} \mathrm{C}$. This effect should be considered in kinetic parameter estimation. The yield is not too high $^{2}$ and increases with temperature, as expected. The " $1^{\text {st }}$ crystallinity at zero bar hydrogen", $\mathrm{Xc}_{1}$ is always higher than $\mathrm{Xc}_{2}$, but does not reach $70 \%$ in all these experiments. The maximum $\mathrm{Mw}$ is in the range of 1 Million $\mathrm{g} / \mathrm{Mol}$ and decreases with temperature as one would expect. Interestingly, the difference in the molecular weights between $50^{\circ} \mathrm{C}$ and $70^{\circ} \mathrm{C}$ is very small.

More details will be discussed in the following chapters.

\subsubsection{Polymerization Rate Profiles}

All the reaction rate profiles shown in Figure 4.1 show an "induction" period that increases with increasing temperature. For better interpretation of rate profiles as shown in Figure 4.1, for example, one should realize of the following: Pneumatic catalyst injection with hexane, which is sprayed through the gas-phase within one second, disturbs the initially reached perfect equilibrium between the gas and liquid phases in the reactor before the catalyst can start the reaction.

As a result, there is a double effect of this hexane spraying:

- cooling down the gas-phase and

- monomer sorption by the fresh hexane.

During the residence time of the small hexane droplets in the gas-phase, an immediate pressure drop occurs that depends on the amount of (cold) hexane used. If this pressure drop is large enough, then the mass flow meter starts to indicate monomer consumption. This small

\footnotetext{
${ }^{1}$ defined as the ratio of the weight average molecular weight to the number average molecular weight measured by GPC

${ }^{2}$ but reasonable for the yield of this gas phase polymerization catalyst used in slurry
} 
consumption is "physical", but is usually interpreted as a "chemical" reaction; however it needs to be discussed when looking at the early stage behaviour.

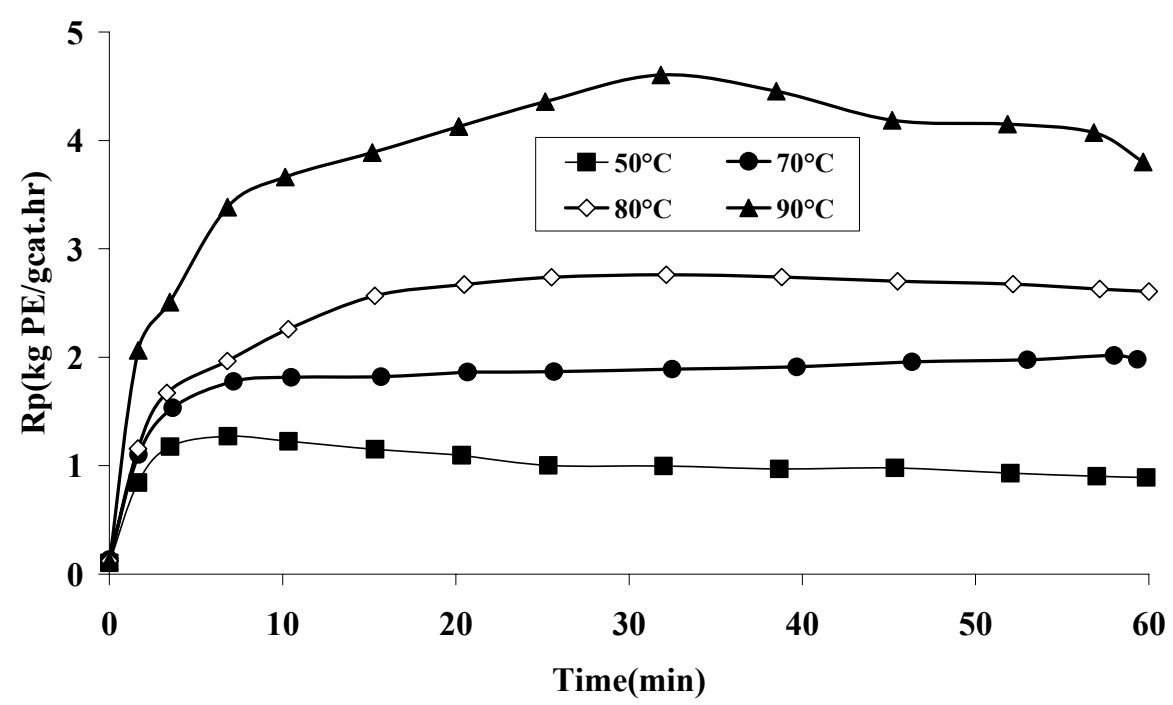

Figure 4.1-Reaction rate-profile of $1^{\text {st }}$ series experiments

A somewhat delayed secondary effect of the fresh hexane injection is the dilution of the monomer-saturated hexane. This lowering of the monomer concentration in the liquid phase is negligible if the equilibrium monomer concentration in the liquid phase is low and/or if the amount of hexane injected is small compared to the volume of the slurry, but it should be taken into account if large amounts of hexane at high monomer pressures are injected.

Real chemical monomer consumption occurs when the catalyst reaches the monomersaturated liquid phase. This is a 3-step process:

1. First, after catalyst injection, the monomer concentration in hexane becomes lower than the equilibrium concentration.

2. As a consequence, monomer transport from the gas phase to liquid phase to compensate for the differences. This transport decreases the gas phase pressure.

3. The monomer mass flow meter starts if a critical pressure below the set point is reached.

Furthermore, to interpret the results in Figure 4.1, we recall that: a potentially active site becomes a real active site if two conditions are met. First, the titanium atom must be located on the surface of a $\mathrm{MgCl}_{2}$ crystal. Second, the generation of new active sites requires internal fragmentation of the catalyst followed by complexation of the new sites with co-catalyst. This complexation can only happen after co-catalyst diffusion to the new active sites occurs.

At $50^{\circ} \mathrm{C}$, it seems, both conditions are given after a very short initial period for all potential sites, with the maximum polymerization rate being reached after about 2 minutes. At $90^{\circ} \mathrm{C}$, the maximum rate is reached after about 30 minutes. Does it take so long to fragment the catalyst completely or does it take 30 minutes for diffusion and complexation at $90^{\circ} \mathrm{C}$ or are both processes responsible? To what extent? We will come back to those questions later, which contribute substantially to the GRAF theory we are developing in this work. 
Table 4.1 summarizes the experimental conditions and the properties of the polymer produced. For a more accurate kinetic analysis, we need to look at the polymerization rate modelling. The following equation is widely applied to express monomer consumption:

$$
\begin{aligned}
R_{m}=- & k_{p} \times M \times N^{*} \\
& M-\text { monomer concentration near the active sites, mol/L } \\
& N^{*} \text { - number of active sites divides by Avogadro's number, mol } \\
& R_{m} \text { - monomer consumption rate, } \mathrm{mol} / \mathrm{h} \\
& k_{p}-\text { propagation constant }{ }^{1}, \mathrm{~L} / \mathrm{mol} \mathrm{h}
\end{aligned}
$$

The molar number of active sites $N^{*}$ depends on fragmentation of the $\mathrm{MgCl}_{2}$ and activation of the titanium by the co-catalyst; therefore we introduce the activation function $f_{a}^{2}$ :

$$
N^{*}=f_{a} N_{T i}
$$

where, $N_{T i}$ is the molar number of Ti atoms in the reactor, which can be calculated from the catalyst mass $m_{\text {cat }}$ injected:

$$
\begin{aligned}
N_{T i}= & m_{c a t} y_{T i} / M_{T i} \\
& y_{T i} \text { - mass fraction of Titanium in the catalyst } \\
& M_{T i} \text {-molar mass of Titanium }
\end{aligned}
$$

The polymerization rate measured in $\mathrm{kg} / \mathrm{gcat} . \mathrm{hr}$ is:

$$
R_{p}=-R_{m} M_{M} / m_{c a t}
$$

Additionally, we assume the monomer concentration, $M$, near the active site to be proportional to the equilibrium bulk concentration of the monomer in hexane:

$$
M=K M_{b} \quad \text { or } \quad M=K C_{C 2} / M_{M}
$$

where $C_{C 2}$ is the equilibrium bulk monomer concentration in hexane, $\mathrm{g} / \mathrm{L}$.

Combining (4.1) and (4.5) we get:

$$
R_{p}=K_{p} C_{C 2}
$$

with the "constant" $K p$ :

$$
K_{p}=k_{p} K f_{a} y_{T i} / M_{T i}
$$

It becomes clear from equation (4.7) and during the derivation of (4.6) how many assumptions are implemented in this model, especially if one takes into account the co-

\footnotetext{
${ }^{1}$ Averaged over the number of different active sites - this ZN catalyst is multi-site catalyst.

${ }^{2} f_{a}$ starts at zero and grows, but the maximum value can be quite small - only about $1 \% \ldots 10 \%$ of all $\mathrm{Ti}$ atoms are considered to be really active; however, this number is extremely uncertain and is hard to estimate
} 
existence of three phases: gas, liquid, and particle; the third exhibiting a very complex and quickly changing arrangement of pores, amorphous polymer and crystalline polymer.

Working under isobaric-isothermal reaction conditions and assuming a very rapid monomer mass transfer between all relevant phases, we can rewrite equation (4.6) to (4.8):

$$
K_{p}=R_{p} / C_{C 2}
$$

We can estimate the activation energy of this modified "propagation constant". Assuming an Arrhenius-type temperature dependence of the modified propagation constant given in equation (4.9):

$$
K_{p}=K_{P 0} \exp \left(-E_{a, p} / R T\right)
$$

and substituting it in equation (4.8), one can derive Equation (4.10) in which, $K p_{0}$ is a pre-exponential factor, $E_{a, p}$ is the activation energy for the propagation, $R$ is the gas constant and $T$ is temperature:

$$
R_{p} / C_{C 2}=K_{P 0} \exp \left(-E_{a, p} / R T\right)
$$

After rearrangement of equation (4.10) and its logarithm, we are able to describe the experimental data from the experiments for finding the activation energy of the propagation by equation (4.11):

$$
\operatorname{Ln}\left(R_{p} / C_{C 2}\right)=-\left(E_{a, p} / R T\right)+\operatorname{Ln}\left(K_{P 0}\right)
$$

For the 1 st series ${ }^{1}$, the activation energy, $E_{a, p}(44.92 \mathrm{~kJ} / \mathrm{mol})$ and the pre-exponential factor, $K p_{0}(2.52 \mathrm{e} 6)$ can be obtained from the slope and the y-intercept of the plot line of $\operatorname{Ln}\left(R p / C c_{2}\right)$ versus $1 / T$ as shown in Figure 4.2.

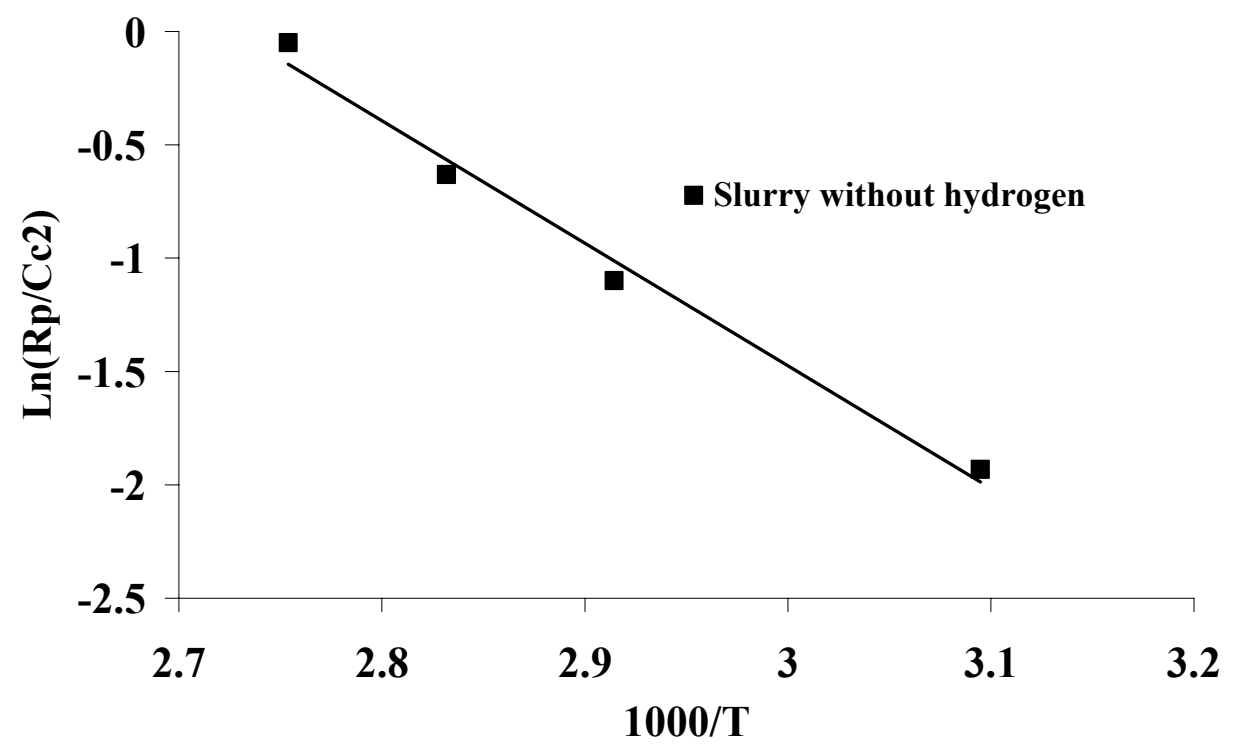

Figure 4.2-Arrhenius plot for estimation of $E_{a, p}$ based on the results listed in Table 4.1 and obtained from equation (4.5) for the temperature series

\footnotetext{
${ }^{1}$ the plateau activity is used as $\mathrm{Rp}$
} 


\subsubsection{Molecular weight and crystallinity}

Following simplest-possible kinetic model, the termination probability $\mathrm{q}$ of the instantaneous polymer produced by a single site catalyst can be expressed by:

$q=k_{1}+k_{2} H_{2} / M$

which relates to the average molecular weight by:

$M_{n}=M_{M} / q$

$M_{w}=2 M_{M} / q \quad \mathrm{M}_{\mathrm{w}}=2 \mathrm{M}_{\mathrm{M}} / \mathrm{q}$

$M_{M}$-molar mass of monomer

Exhibiting a polydispersity index of exactly $\mathrm{PD}=2$.

Deviations from $\mathrm{PD}=2$ can be explained in term of the multi-site behaviour of $\mathrm{ZN}$ catalysts and - additionally - in term of temperature and concentration differences during the course of the reaction. Furthermore, $k_{1}$ and $k_{2}$ are ratios of kinetic constants with positive activation energies - both constants increase with temperature and the molecular weight usually decreases with increasing temperature. One should also take into account that these constants probably have different activation energies for different active sites. However, (1) more mass and heat transfer limitation at high temperatures; and (2) uneven deactivation of the different sites can compensate substantially the temperature effect of $\mathrm{k}_{1}$ and $\mathrm{k}_{2}$.

In our case , (1) in absence of $\mathrm{H}_{2}$; and (2) assuming no mass and heat transfer limitations, one should find a steadily decreasing molecular weight of the polymer produced with increasing temperature if the deactivation of different sites does not differ too much. However, in the case of a lower deactivation rate of high-molecular weight producing sites (compared to low molecular weight producing sites) it can happen that this difference in deactivation leads to a higher molecular weight at higher temperatures. This seems to be the case, as shown in Table 4.1, the molecular weight decreases from 50 to $80^{\circ} \mathrm{C}$, but increases slightly at $90^{\circ} \mathrm{C}$. Different activation energies for $q$ of different active sites can also contribute to this effect: at higher temperatures, lower-Mw producing sites move "faster" to the left of the MWD than higher$\mathrm{Mw}$ producing sites, and their contribution can increase. This would explain both the shift to the left and the increasing PD.

Another explanation is the presence of other transfer reactions. If the transfer reaction to the co-catalyst and/or to hexane cannot be excluded completely, then swelling of the polymer with hexane and the mass transfer of the co-catalyst can influence the molecular weight characteristics and its temperature dependence. 
The PD changed very little between 3.59 and 3.65 as the temperature increased from $50^{\circ} \mathrm{C}$ to $70^{\circ} \mathrm{C}$ followed by 4.1 at $80^{\circ} \mathrm{C}$ and 3.6 at $90^{\circ} \mathrm{C}$. The change in molecular weight became more distinct from MWD data, as shown in Figure 4.3.

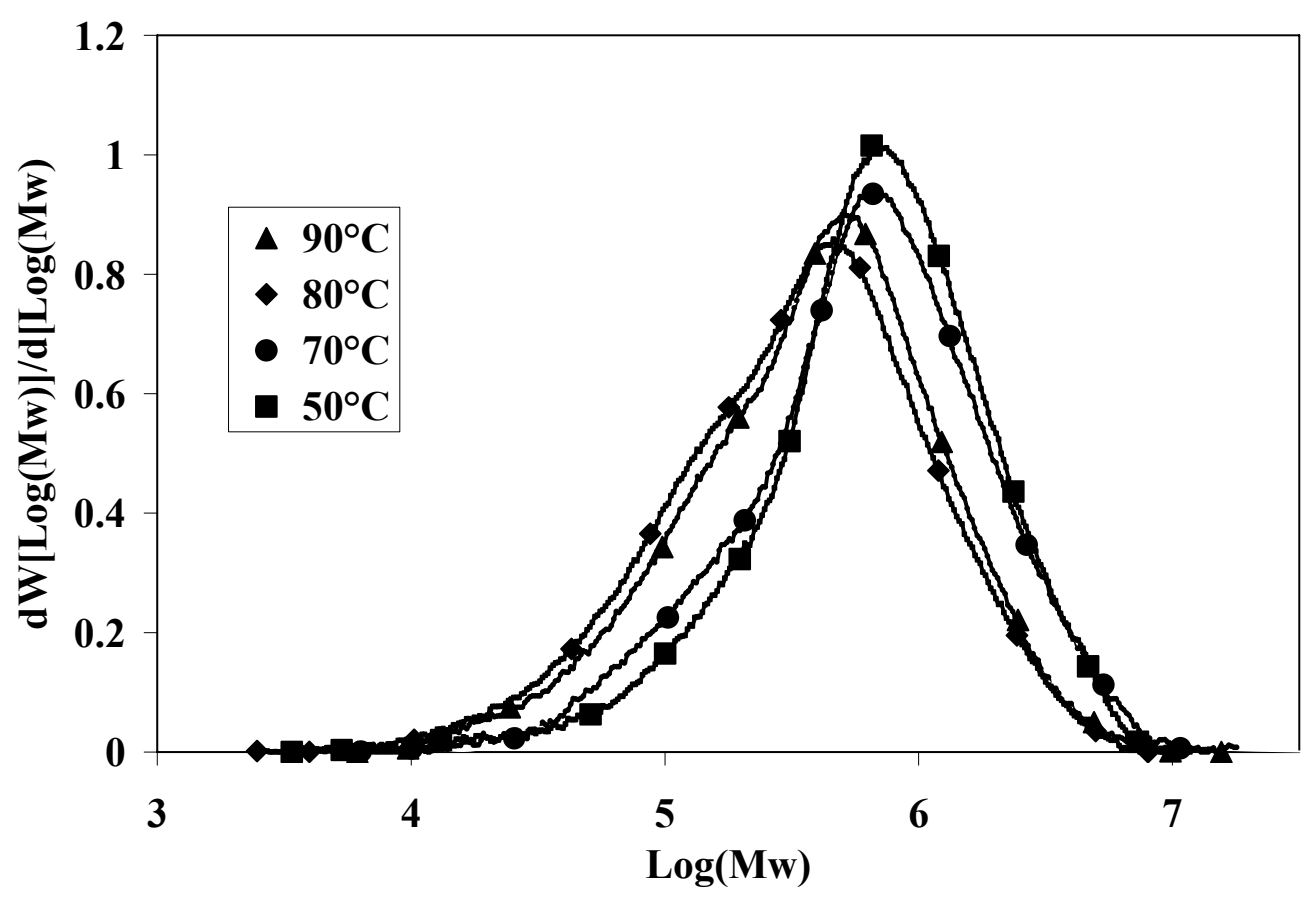

Figure 4.3-Temperature influence on MWDs of polyethylene produced in $1^{\text {st }}$ series

In terms of crystallinity, Table 4.1 shows that both crystallinities (first and second) increased roughly with increasing temperature. Figure 4.4 shows the first and second heating thermogram by DSC for produced polymers of the first series. This changing is in good agreement with the known relation between crystallinity and the molecular weight of polyethylene: lower molecular weight PE crystallizes more rapidly than high molecular weight PE. Clearly, the chain mobility of smaller chains is higher and enables a faster and more complete formation of parallel (crystalline) structures. Of course, chain mobility depends mainly on two conditions: the first is the chain length, as previously discussed; the second is the micro-viscosity of the material through which the chain must move to form crystals.

Now, it is interesting that, for all temperatures, the first crystallinity is higher than the second one: $\mathrm{Xc}_{1}>\mathrm{Xc}_{2}$. This means that the crystallinity of the polymer after polymerization under polymerization conditions - is higher than that after melting and re-crystallization. How should this be interpreted? This also reported for UHMW PE by many researchers [70, 71]. We regard micro-viscosity as the dominant factor: at lower temperatures, Table 4.1 show that at $50^{\circ} \mathrm{C}$, the viscosity of the polymer matrix is lower, because of the sorption effect of hexane (and monomer) sorption at lower temperature that over-compensates the direct viscosity-increasing effect of lower temperatures. Therefore, the crystallinity difference between $1^{\text {st }}$ and $2^{\text {nd }}$ crystallinity is highest at $50^{\circ} \mathrm{C}$. 
A)

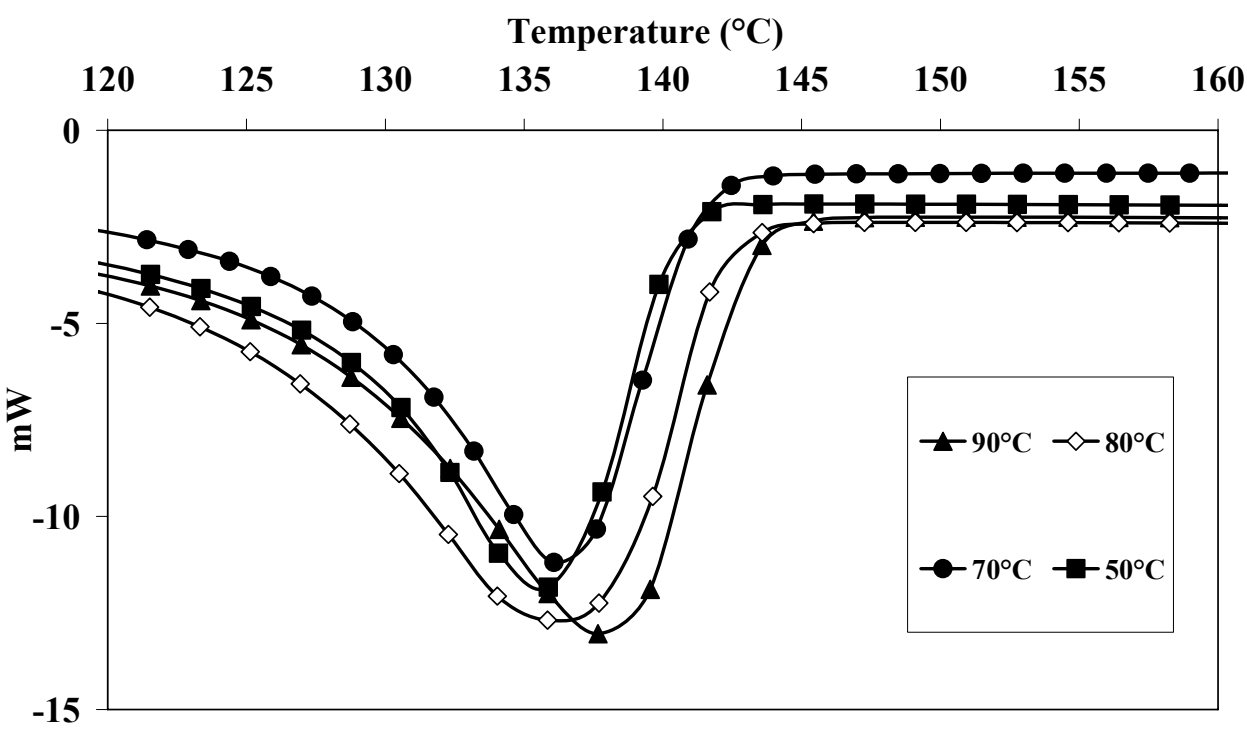

B)

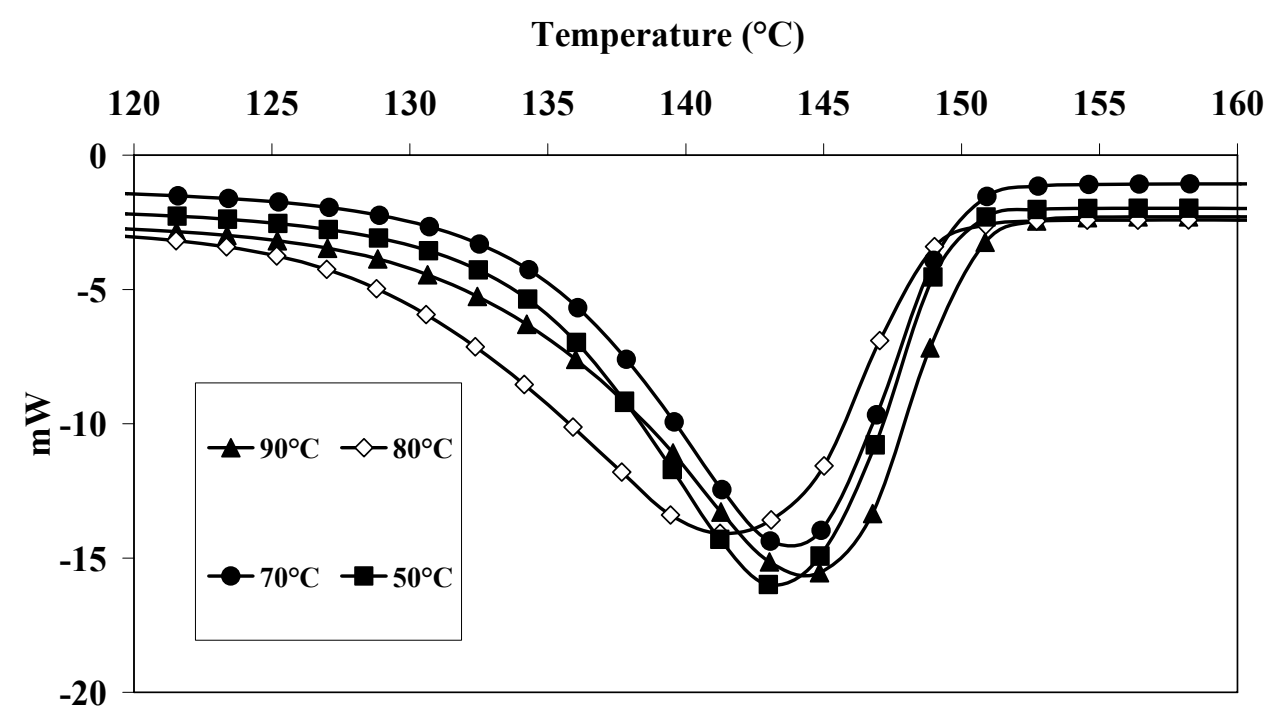

Figure 4.4-DSC scan for HDPE samples obtained in temperature series slurry ethylene polymerization without hydrogen (heating rate: $10 \mathrm{C} / \mathrm{min}$, $\mathrm{N} 2$ Atmosphere) $(\mathrm{A}): 1^{\text {st }}$ heating (B): $2^{\text {nd }}$ heating

\subsubsection{Morphology}

No fines generation can be seen in PSD as the temperature rises from $50^{\circ} \mathrm{C}$ to $90^{\circ} \mathrm{C}$; see Figure 4.5. However, larger particles were produced at lower temperature $\left(50^{\circ} \mathrm{C}\right)-$ the upper part of the normalized PSD moves to the right at lower temperatures. Is the stickiness (lower matrix viscosity) of the particles - swollen with more hexane at lower temperatures responsible for this effect? However, the change in PSD is not dramatic. 


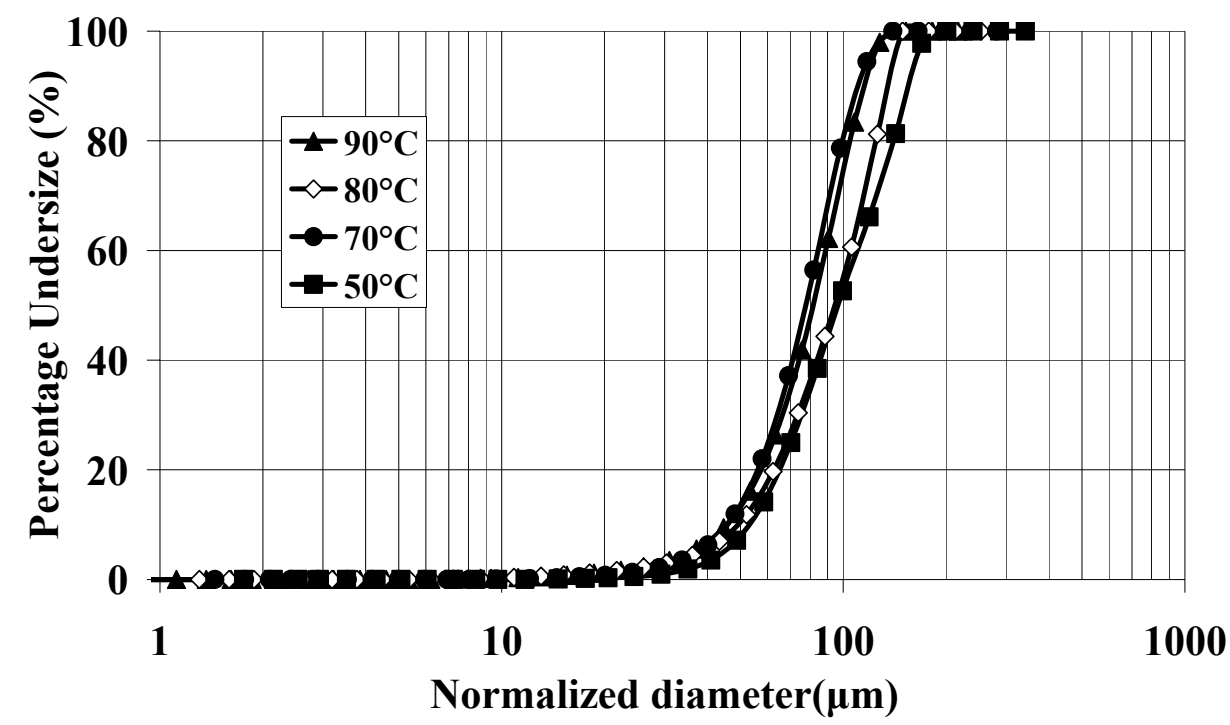

Figure 4.5-The influence of temperature on cumulative PSD profiles normalized with the yield for the $1^{\text {st }}$ series

Figure 4.6 shows the TEM image of the same polymer sample. As can be seen, crystalline regions and amorphous regions are almost uniformly distributed in the whole structure. This uniform distribution is in good agreement with the level of crystallinity obtained with DSC (60\%). The crystal size is relatively small.

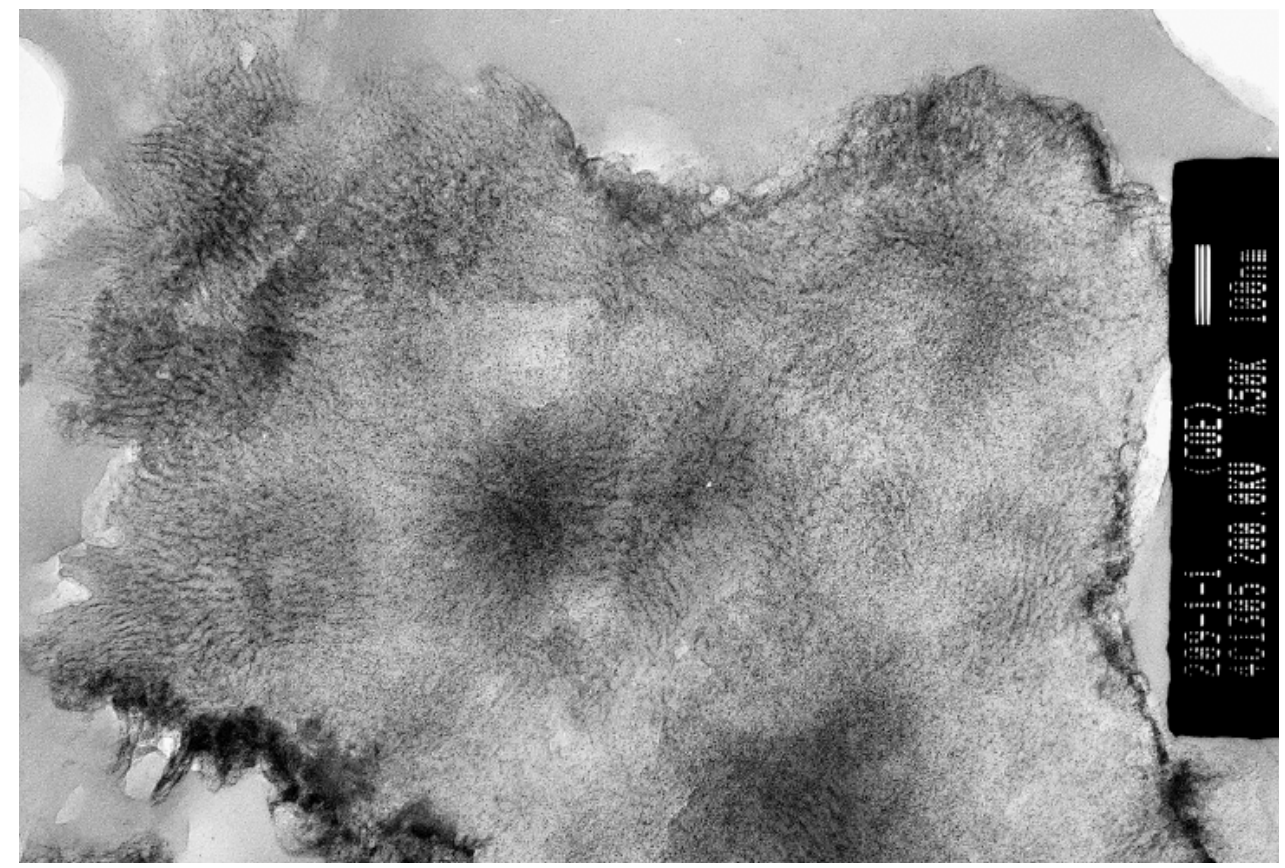

Figure 4.6-The TEM image of the polymer obtained at run 3 
The surface morphology of the polymer produced at $80^{\circ} \mathrm{C}$ is shown in Figure 4.7 with different resolution. The surface of the particles is to a large extent "open", such that many pores are visible. The fibrillar structure - at the highest magnification - indicates the expansion of the solid phase under growth stress.

A)

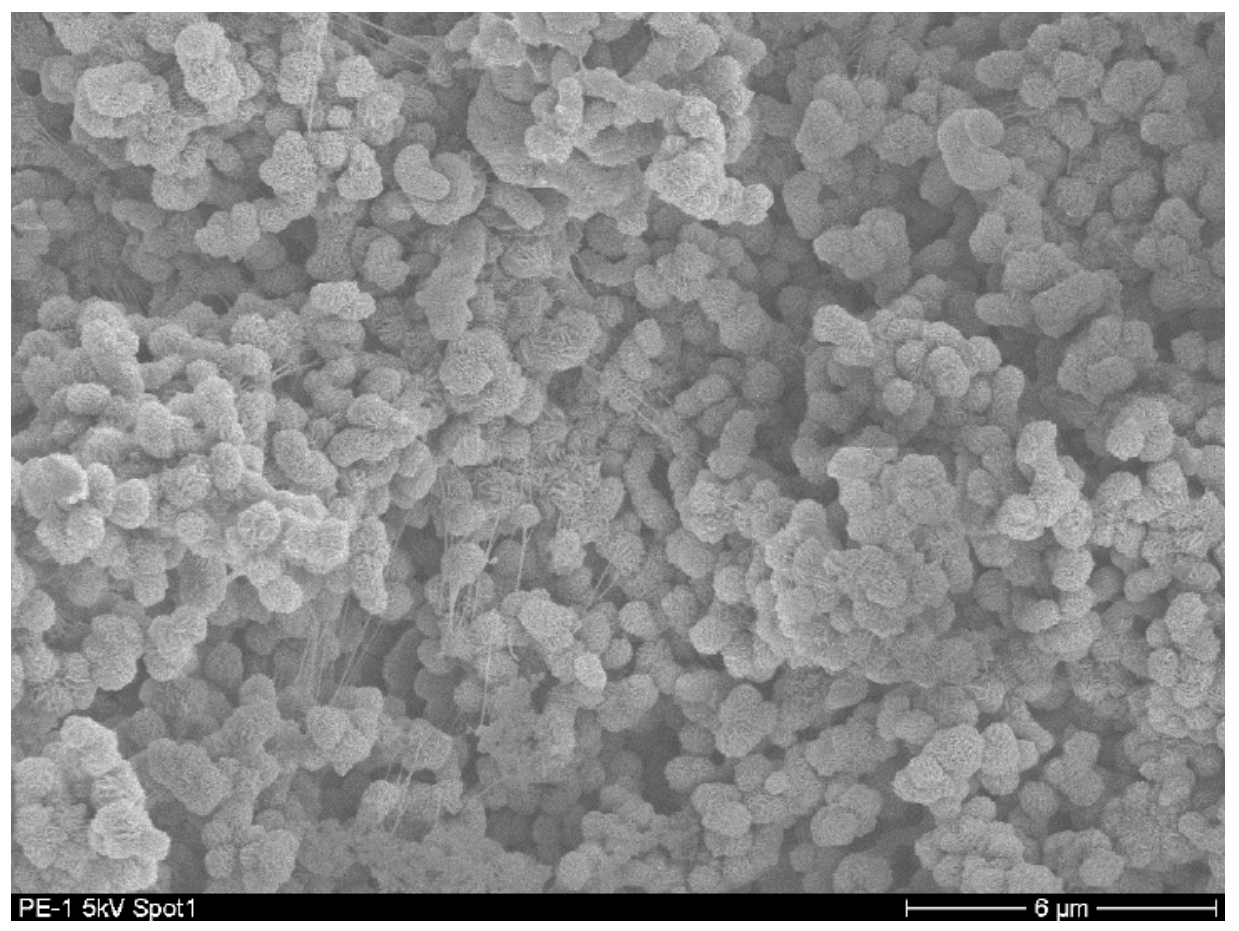

B)

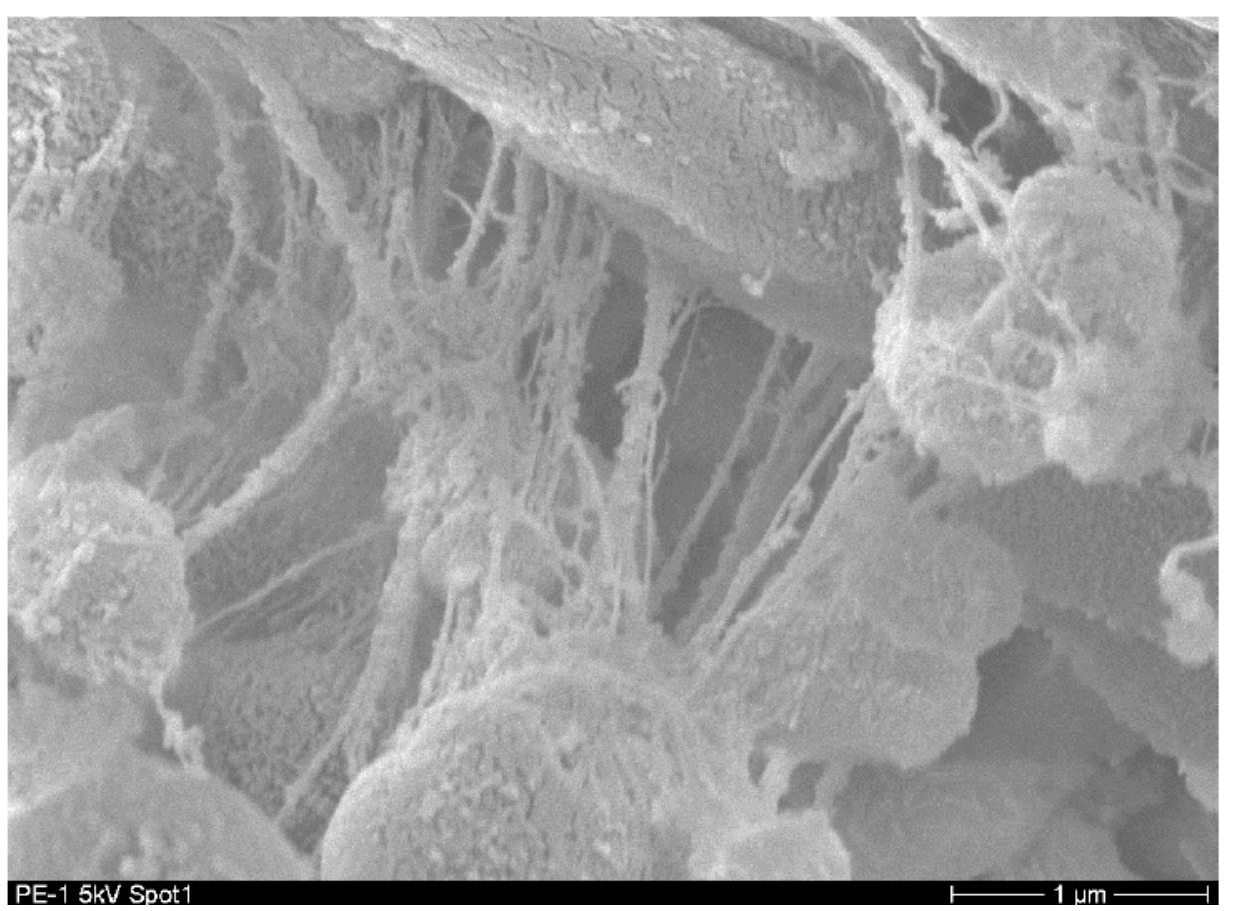

Figure 4.7-The SEM images, experiment run 3 A)with $6 \mu \mathrm{m}$ resolution B) with $1 \mu \mathrm{m}$ resolution 


\subsection{Slurry Polymerization in the Presence of Hydrogen}

Table 4.2 summarizes the experimental conditions and the results obtained for $2^{\text {nd }}$ series in the presence of hydrogen ( 2 bar). All process conditions are the same as for the previous series, except for the hydrogen partial pressure, which is 2 bar.

Comparing Table 4.1 and Table 4.2, it becomes clear that the presence of hydrogen:

- decreases the solubility of ethylene slightly $(6.4 \mathrm{~g} / \mathrm{L}$ compared to $6.89 \mathrm{~g} / \mathrm{L}$ at $\left.90^{\circ} \mathrm{C}\right)$

- increases the yield, except at $90^{\circ} \mathrm{C}$

- decreases the molecular weight and consequently increases the crystallinities $\mathrm{Xc}_{1}$ and $\mathrm{Xc}_{2} ; \mathrm{Mw}$ for all runs is in the range of 130 to $200 \mathrm{~kg} / \mathrm{mol}$ which is almost 5 times lower than those for the $1^{\text {st }}$ series,

- leads to a strong broadening of the MWD as indicated by the increase in PD.

Further results are:

- the yield increases with temperature up to $80^{\circ} \mathrm{C}$ and then decreases at $90^{\circ} \mathrm{C}$

- $\mathrm{Xc}_{1}$ for all runs is lower than $\mathrm{Xc}_{2}$ which is in reverse order compared to what we observed in absence of hydrogen; the difference is always about $5 \%$

- $\mathrm{Xc}_{2}$ is higher than $70 \%$ for all runs, reaching very high values for run $8\left(80^{\circ} \mathrm{C}\right)$ and run $9\left(90^{\circ} \mathrm{C}\right)$; the trend of increasing the crystallinity with increasing temperature of reaction is in good agreement with decreasing molecular weight, as described previously in Chapter 4.3.1.

Table 4.2-Operating conditions and produced polymer properties of $2^{\text {nd }}$ series

\begin{tabular}{lccccccccc}
\hline Run & $\begin{array}{c}\mathrm{T} \\
\left({ }^{\circ} \mathrm{C}\right)\end{array}$ & $\begin{array}{c}\mathrm{Cc}_{2} \\
\mathrm{~g} / \mathrm{L}\end{array}$ & $\begin{array}{c}\mathrm{C}_{\mathrm{H} 2} \\
\mathrm{~g} / \mathrm{L}\end{array}$ & $R_{p a}$ & $\begin{array}{c}\mathrm{Xc}_{1} \\
\%\end{array}$ & $\begin{array}{c}\mathrm{Xc}_{2} \\
\%\end{array}$ & $\begin{array}{c}\mathrm{Mw} \\
(\mathrm{kg} / \mathrm{mol})\end{array}$ & $\begin{array}{c}\mathrm{Mn} \\
(\mathrm{kg} / \mathrm{mol})\end{array}$ & $\begin{array}{c}\mathrm{PD}= \\
\mathrm{Mw} / \mathrm{Mn}\end{array}$ \\
\hline 5 & 60 & 6.4 & 0.027 & 1.5 & 67.7 & 72.5 & 200 & 22.4 & 8.9 \\
6 & 65 & 6.1 & 0.027 & 2.6 & 67.1 & 72.2 & 190 & 17.9 & 10.6 \\
7 & 70 & 5.8 & 0.027 & 2.5 & 66.6 & 71 & 153 & 15.7 & 9.7 \\
8 & 80 & 5.2 & 0.027 & 4.4 & 71.3 & 76.6 & 140 & 15.2 & 9.2 \\
9 & 90 & 4.7 & 0.027 & 2.7 & 76.5 & 82.5 & 137 & 13.4 & 10.2 \\
\hline
\end{tabular}

$R_{p a}$ is expressed as (kg PE/gcat.hr)

\subsubsection{Polymerization Rate Profiles}

Figure 4.8 shows those reaction rate profiles that show no deactivation within 1 hour and for the polymerization rate increases at temperature between 60 and $80^{\circ} \mathrm{C}$, but decreases rapidly at $90^{\circ} \mathrm{C}$. 


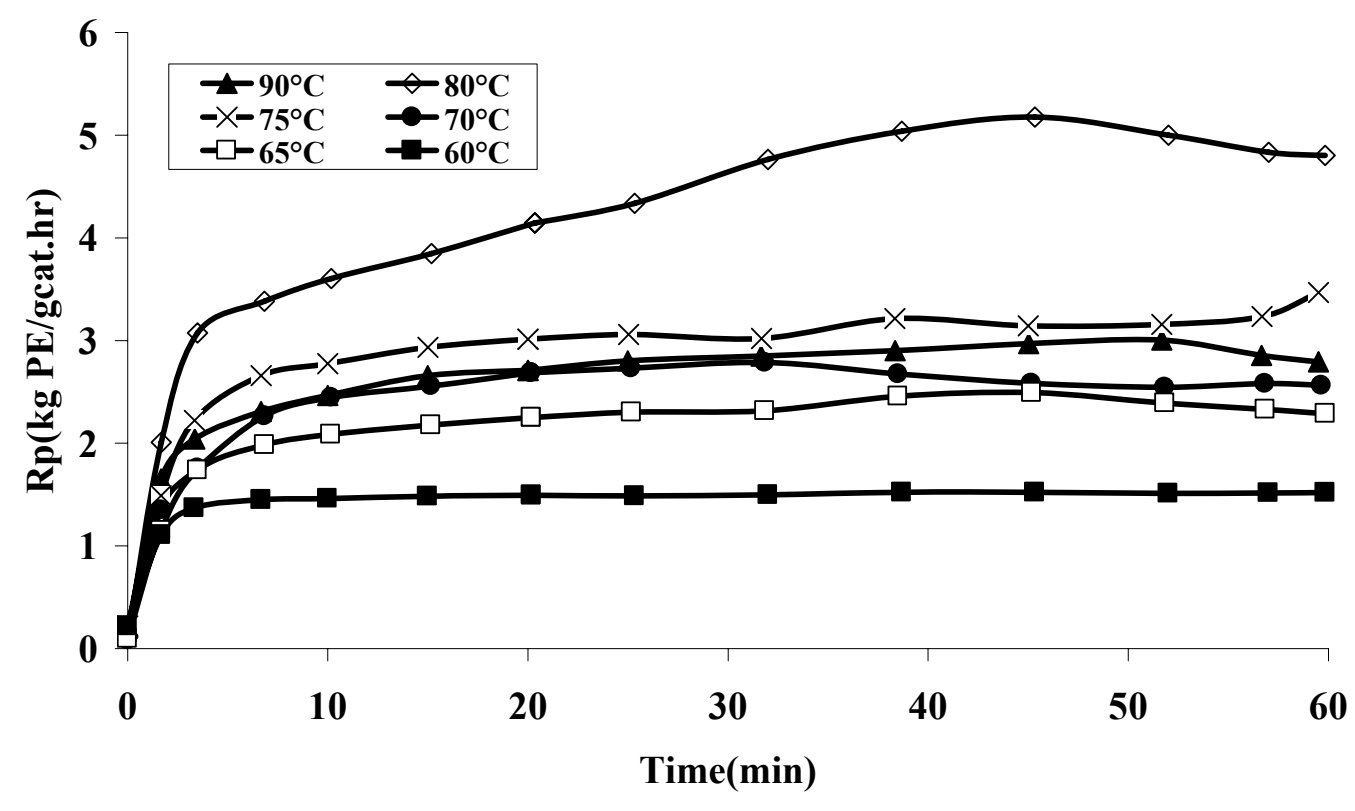

Figure 4.8-Slurry polymerization of ethylene in the presence of hydrogen: rate profile

What is the reason for this sudden rate drop at $90^{\circ} \mathrm{C}$ ?

Local hot spots and mass transfer limitation by particle melting - as discussed by for example W.H. Ray et al (1997) [22] for the ethylene polymerization in the gas-phase - are hardly possible in slurry. One possible explanation is the high crystallinity observed at higher temperatures. Monomer diffusion through crystals is negligible [72]. Because of this "crystal barrier", the monomer concentration near the active sites decreases and the mass transfer limitation on the propagation reaction becomes obvious.

Deactivation by reversible reaction of the active site with hydrogen, as first suggested by Kissin [18] , can serve as another explanation:

$$
\mathrm{C}^{*}+\mathrm{H}_{2} \longleftrightarrow \mathrm{C}_{\mathrm{H} 2}^{*}
$$

where $\mathrm{C}^{*}$ is the number of active centers and $\mathrm{C}_{\mathrm{H} 2}^{*}$ is the temporary deactivated centers by hydrogen. In the temperature range 50 to $80^{\circ} \mathrm{C}$, the propagation rate increase dominates, whereas at higher temperatures the equilibrium moves too much to the right forming non-active sites.

To estimate of $\mathrm{Kp}_{0}$ (the pre-exponential factor) and Ea,p (the activation energy) of the $2^{\text {nd }}$ series, we used equation (4.11). We excluded the data for $90^{\circ} \mathrm{C}$. Figure 4.9 show the Arrhenius plot and the data obtained for $K p_{0}(3.37 \mathrm{e} 8)$ and $E_{a, p}(58.9 \mathrm{~kJ} / \mathrm{mol})$ for the second series. As can be seen, the activation energy and the pre-exponential factor for $2^{\text {nd }}$ series (with hydrogen) are higher and lower respectively when compared to $1^{\text {st }}$ series (without hydrogen). 


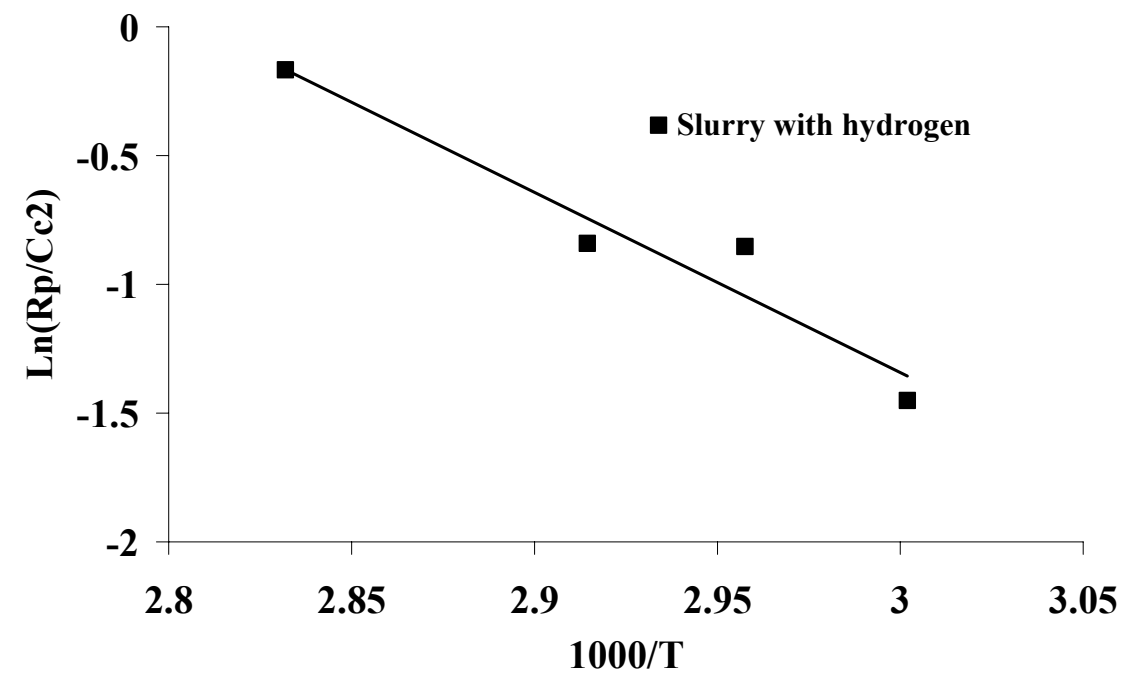

Figure 4.9-Arrhenius plot for estimation of $E_{a, p}$ based on the results obtained by Table 4.2 and equation (4.5) for the temperature series

\subsubsection{Molecular Weight and Crystallinity}

For the catalyst system used in this study, Table 4.2 shows that hydrogen is a major chain-length regulating agent for the catalyst used, as expected for most Z-N catalysts. This is confirmed by Figure 4.10. Interestingly, the MWDs of produced polymers at both $80^{\circ} \mathrm{C}$ and $90^{\circ} \mathrm{C}$ show a high molecular weight shoulder. This shoulder can be attributed to the fact that the high-molecular weight producing sites show a lower hydrogen response. However, this shoulder is not present at lower temperatures and in absence of hydrogen as shown in Figure 4.3 .

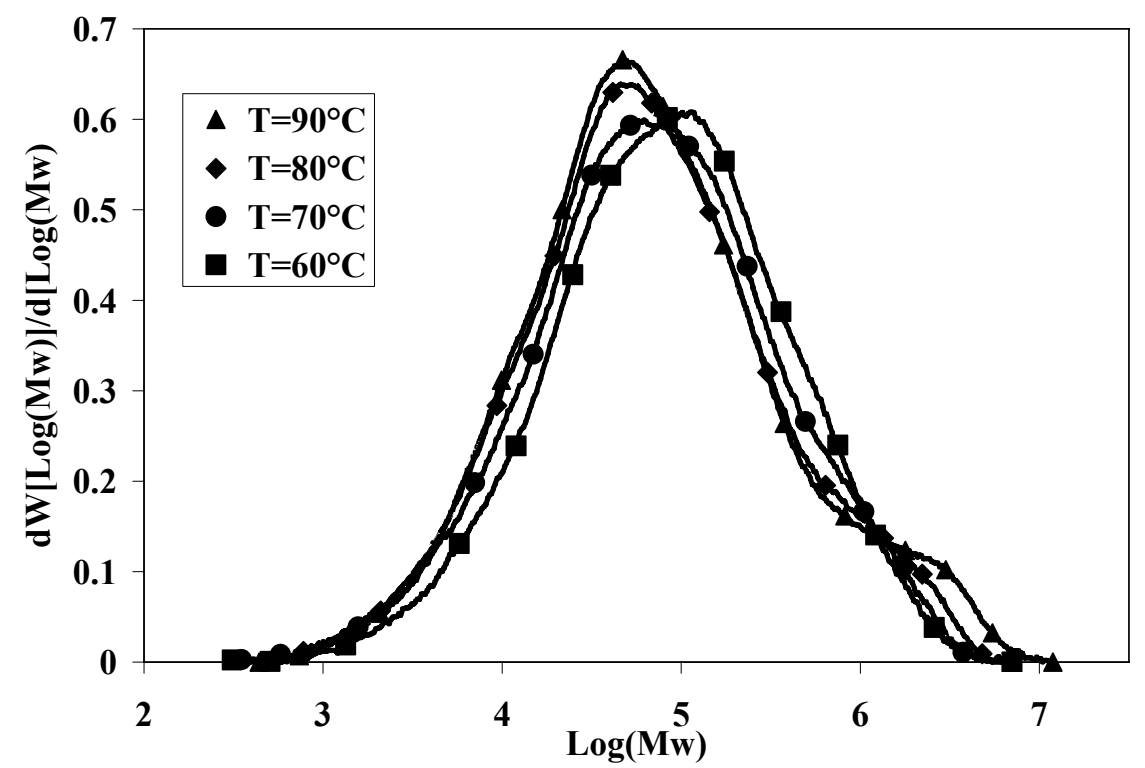

Figure 4.10-Temperature influence on MWDs of polyethylene produced in temperature series slurry homo-ethylene polymerization with hydrogen for gas-phase catalyst at $\mathbf{P}_{\mathrm{C} 2}=\mathbf{2}$ bar and $\mathbf{P}_{\mathrm{H} 2}=\mathbf{2}$ bar. 
The increase in PD caused by the presence of hydrogen can be attributed to the existence of different active sites with different chain-transfer sensitivity to hydrogen. The temperature influence on PD is less strong; only the high Mw shoulder causes a major difference.

Compared to the experiments performed in absence of hydrogen, we found that $\mathrm{Xc}_{2}$ $>\mathrm{Xc}_{1}$ for $2^{\text {nd }}$ series but $\mathrm{Xc}_{2}<\mathrm{Xc}_{1}$ for $1^{\text {st }}$ series; how can this behaviour be explained?

We have to discuss three processes which are characterized by their specific rates:

- chain production

- chain crystallization

- re-crystallization after melting.

In presence of hydrogen, relatively small chains are formed at higher production rates: clearly, these chains do not find time to crystallize to the equilibrium. Melting and (slow-rate) re-crystallization leads to an increase in the folding ability because of a rearrangement of crystallites to a more crystalline structure $\mathrm{Xc}_{2}>\mathrm{Xc}_{1}$.

In the absence of hydrogen, longer polymer chains that cause folding are formed. Melting of the corresponding polymers will decrease the strength of folding ability, leading into a decrease in crystallinity after melting $\mathrm{Xc}_{2}<\mathrm{Xc}_{1}$.

Figure 4.11 shows the $1^{\text {st }}$ and $2^{\text {nd }}$ heating thermograms obtained by DSC for produced polymers in the second series.

A)

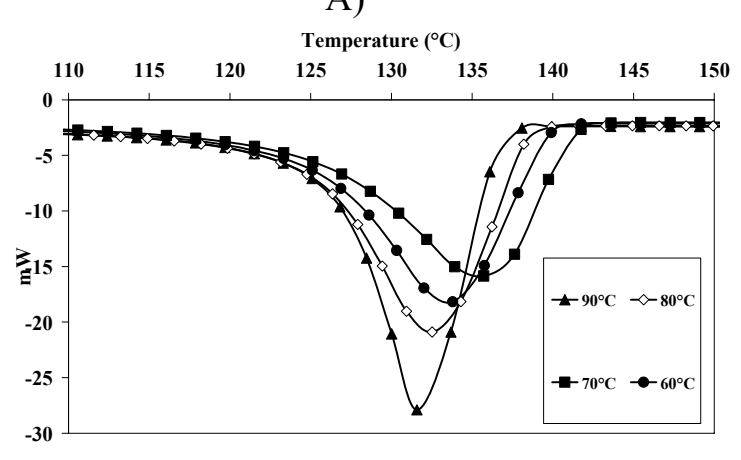

B)

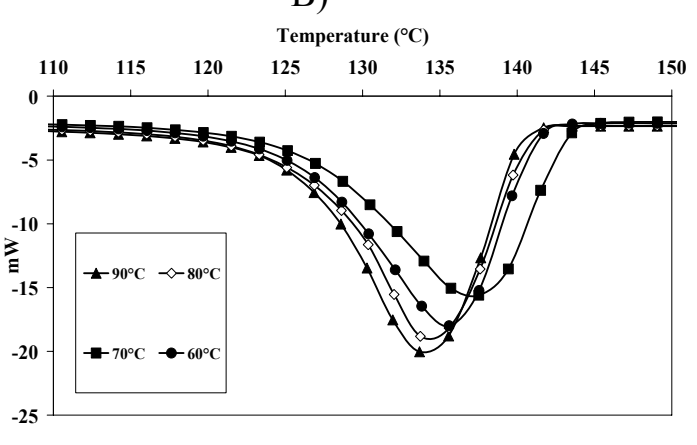

Figure 4.11-DSC scan for HDPE samples obtained in temperature series slurry ethylene polymerization with hydrogen (heating rate: $10 \mathrm{C} / \mathrm{min}, \mathrm{N} 2$ Atmosphere) $(\mathrm{A}): 1^{\text {st }}$ heating (B): $2^{\text {nd }}$ heating

\subsubsection{Morphology}

The influence of the polymerization temperature on the cumulative particle size distribution of the second series normalized with the yield is shown in Figure 4.12. It is interesting that PSD remains unaffected as the polymerization temperature increases up to $70^{\circ} \mathrm{C}$. However, at higher temperatures, we see a remarkable shift of the PSD curves to the left towards lower particle sizes. The percentage of normalized particles with a diameter of $40 \mu \mathrm{m}$ increases from $7 \%$ to $18 \%, 40 \%$ and $50 \%$ as the relevant polymerization temperature 
increases from $70^{\circ} \mathrm{C}$ to $75^{\circ} \mathrm{C}, 80^{\circ} \mathrm{C}$ to $90^{\circ} \mathrm{C}$ respectively. However, this did not happen in absence of hydrogen, which can possibly be explained as follows.

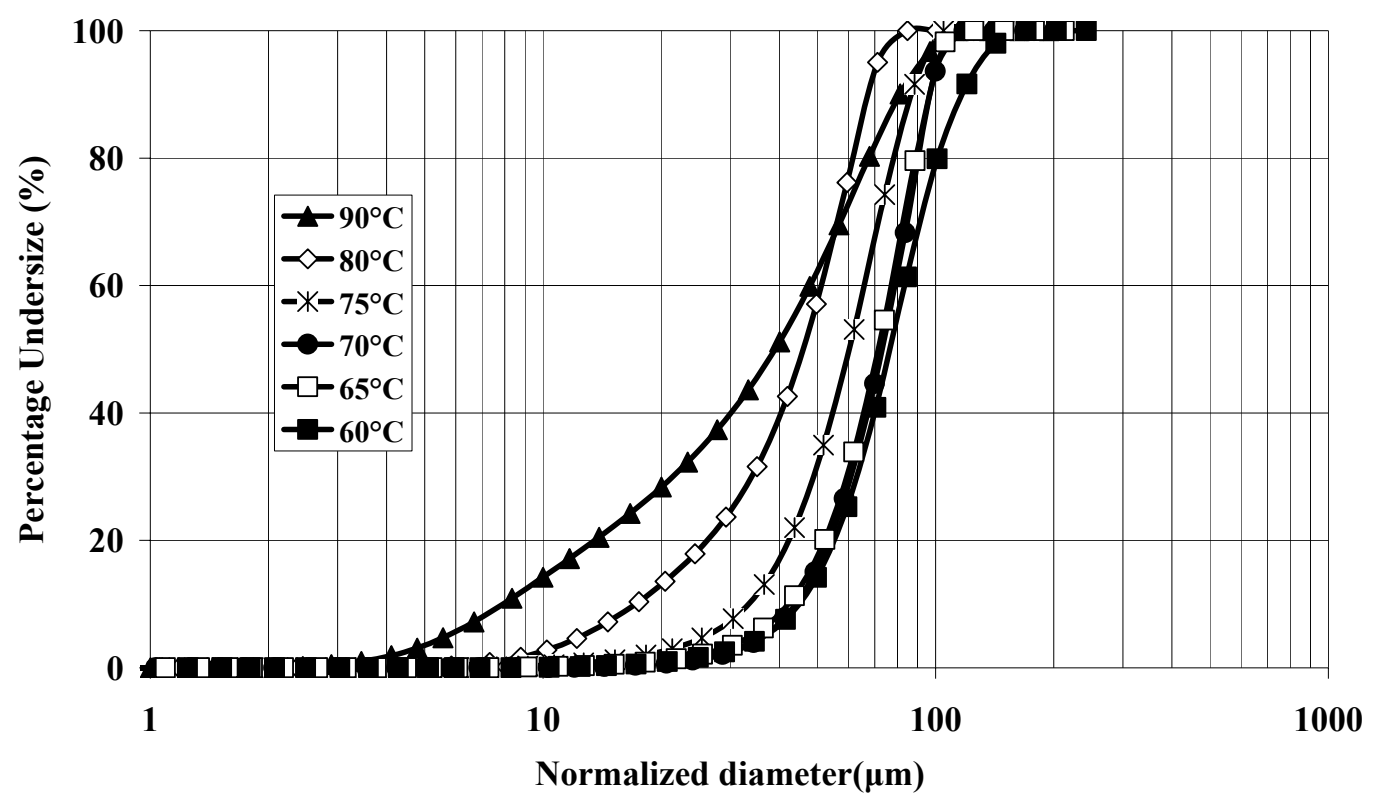

Figure 4.12-The influence of temperature on cumulative PSD profiles normalized with the yield $\left(P_{\mathrm{C} 2}=2 \mathrm{bar} \& \mathrm{P}_{\mathrm{H} 2}=2 \mathrm{bar}\right)$ in slurry homo-ethylene polymerization reaction with the presence of hydrogen

The brittleness of growing particles increases with:

- increasing crystallinity

- increasing growth stress.

Increasing crystallinity results from shorter chains (caused by more hydrogen). Such polymers crystallize faster due to two facts: (1) their mobility is higher compared to long chains (2) they form a matrix (hexane swollen amorphous PE) of lower micro-viscosity through which other polymers can move easier ${ }^{1}$. Furthermore, increasing the temperature has a number of effects:

- the growing polymerization rate leads to increasing growth stress; however, the opposite effect can result at higher temperatures when the polymerization rate decreases with temperature

- the chain mobility of any molecule increases with increasing temperature

- usually the solubility of hexane in PE decreases with increasing temperature; however, in a closed system such as in our batch reactor, the vapour pressure of hexane increases exponentially with temperature and this partial pressure rise increases the concentration of hexane in PE; the viscosity of the polymer matrix decreases enormously and the freshly produced polymer chains can move more easily through the surrounding "solid"; during the chain production, this process cannot be described as a diffusion process.

\footnotetext{
${ }^{1}$ to avoid misunderstanding: we are talking about the mobility and crystallization of PE chains during their production ("in-situ") at a given active site; therefore, the crystallization behavior of these chains can differ substantially from that of dead chains; compare [73, 74].
} 
Taking into account the above "chain of logic", we can conclude that at high hydrogen pressure the tendency to fragmentation (="brittleness") increases and is driven by the following factors:

- low Mw polymer crystallizes more rapidly (smaller chains show a higher mobility and the matrix viscosity decreases)

- an increasing polymerization rate causes an increasing growth stress

- faster fragmentation produces more active sites, and as a result the growth stress increases.

It seems to be out of question now: fragmentation increases the polymerization rate. This is clearly a physical effect in terms of kinetics that should be taken into account if one wants to model polymerization kinetics!

On the other hands, the increasing temperature must have a direct rate-decreasing effect, because the polymerization rate at $90^{\circ} \mathrm{C}$ is much lower than that at $80^{\circ} \mathrm{C}$, despite more complete fragmentation at $90^{\circ} \mathrm{C}$. To discuss this fact, one should appreciate that the molecular weight remains the same at both $80^{\circ} \mathrm{C}$ and $90^{\circ} \mathrm{C}$; it is just that the crystallinity at $90^{\circ} \mathrm{C}$ is higher, as shown in Table 4.2.

It is worth mentioning that this is the crystallinity after one hour of reaction and as discussed in Chapter 3 (section: time series) the real crystallinity is much higher at the early stage of polymerization leading to the production of high brittleness polymer particles.

TEM image of the polymer produced at $60^{\circ} \mathrm{C}$ (see Figure 4.13) shows the existence of more dense and uniform distributed crystalline regions compared to that obtained at the even higher temperature of $80^{\circ} \mathrm{C}$ in the absence of hydrogen (see Figure 4.6), but still the crystal size is small. By increasing the reaction temperature in presence of hydrogen, the size of the crystal increases; see Figure 4.14. We conclude that under a given particle growth stress, big crystals can break much more easily than those of small size. Clearly, this helps to explain the high degree of external fragmentation observed at higher temperatures in presence of hydrogen.

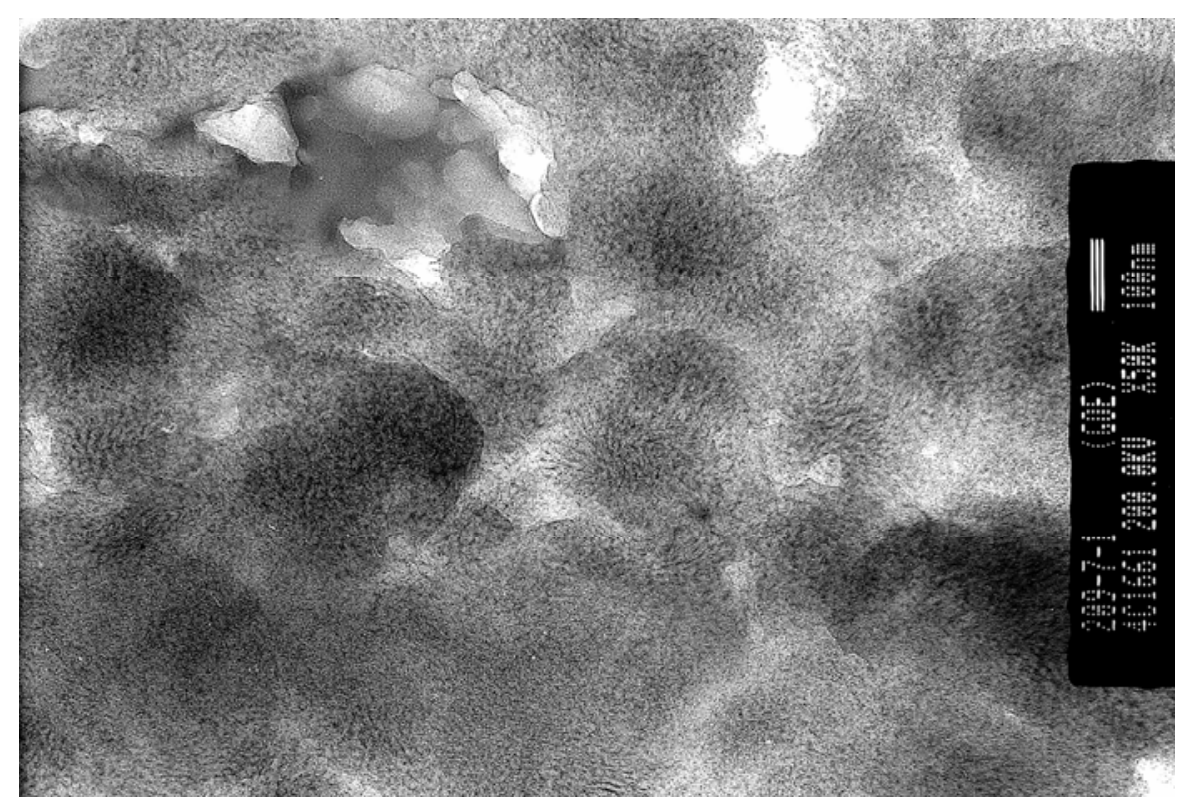

Figure 4.13 -The TEM image of the polymer obtained during run 5 


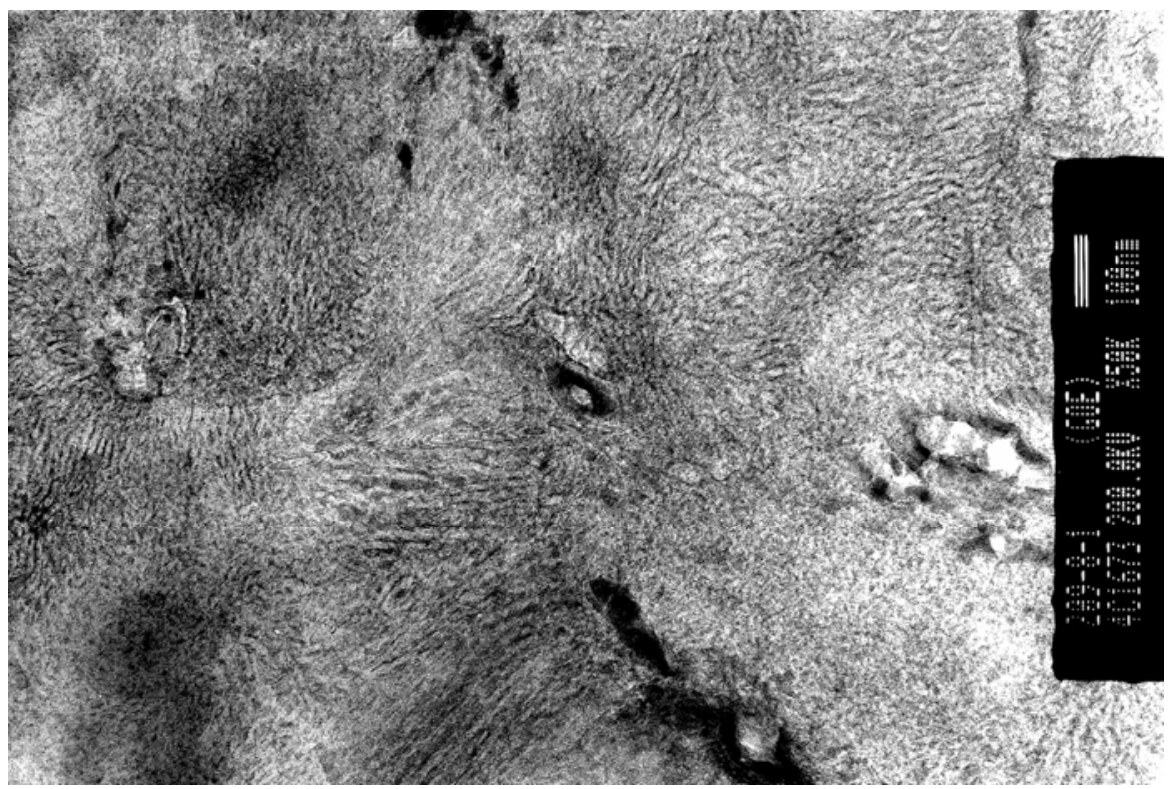

Figure 4.14-The TEM image of the polymer obtained during run 9

A)

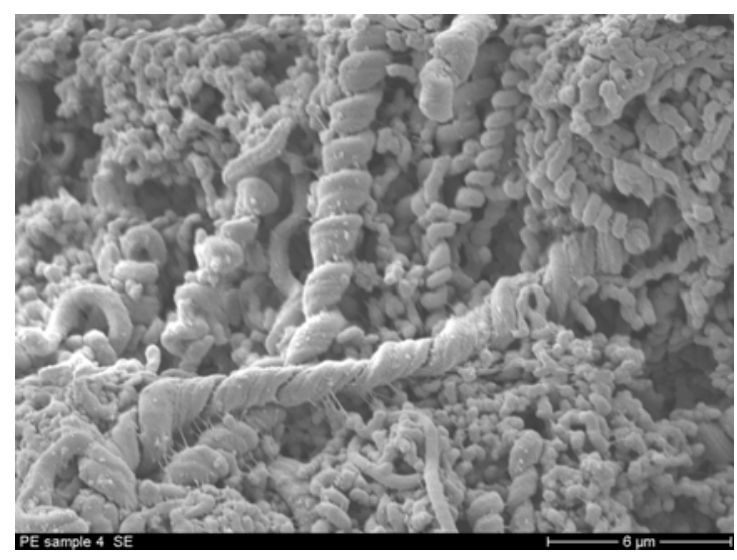

B)

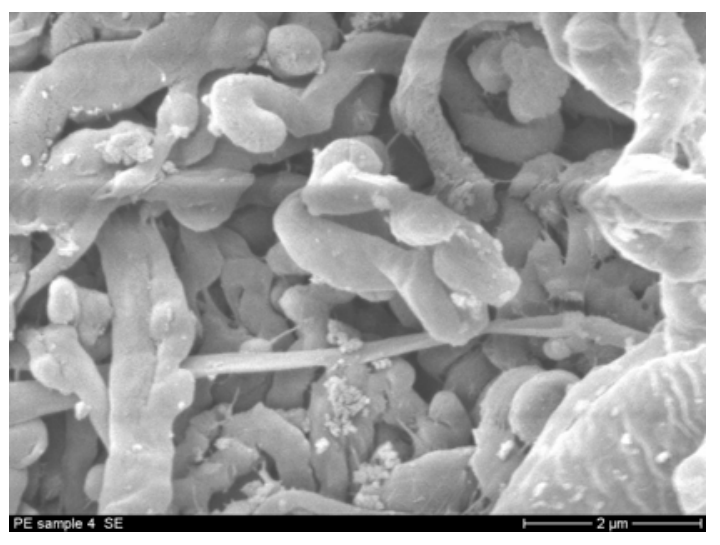

Figure 4.15-The SEM images of the polymer obtained during run 5: A) with $6 \mu \mathrm{m}$ contrast B) with $2 \mu \mathrm{m}$ contrast

Figure 4.15 and Figure 4.16 show the surface morphology of the polymer produced at $60^{\circ} \mathrm{C}$ and $90^{\circ} \mathrm{C}$ respectively at different magnifications. It seems that the surface morphology of the polymer produced at $60^{\circ} \mathrm{C}$ starts with a globular structure and then the wormlike structure grows as the reaction proceeds. Figure 4.15 also shows how some wormlike polymer particles of different diameter and length twisted around each other. On the other hand, the surface morphology of the polymer produced at $90^{\circ} \mathrm{C}$ (see Figure 4.16) is to a large extent irregular with numerous deep cracks on the surface. Such a structure could be due to the high crystallinity obtained. 
A)

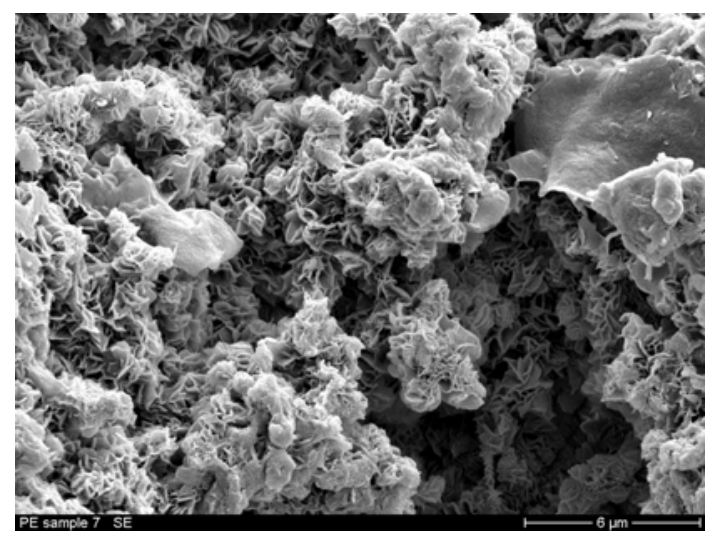

B)

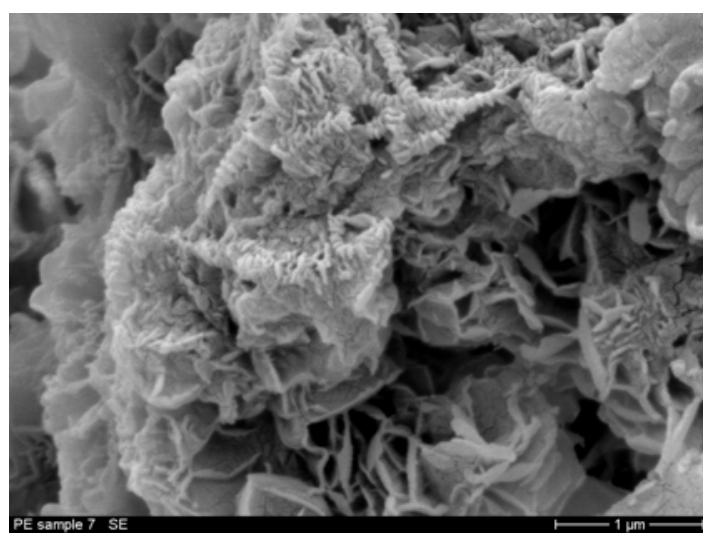

Figure 4.16-The SEM images of the polymer obtained during run 9: A) with $6 \mu \mathrm{m}$ contrast B) with $2 \mu \mathrm{m}$ contrast

\subsection{Gas-Phase Polymerization in the Presence of Hydrogen}

All process conditions remained the same as in the second series, except that instead of hexane, $110 \mathrm{~g}$ salt was used. Therefore, for comparing the differences between the gasphase and slurry polymerization, we can compare the following figures and tables directly:

- $\quad$ Table 4.2 and Table 4.3

- $\quad$ Figure 4.8 and Figure 4.17

- $\quad$ Figure 4.10and Figure 4.19

- Figure 4.11 and Figure 4.20

- $\quad$ Figure 4.12and Figure 4.21

Table 4.3-Gas-phase polymerization of ethylene $\left(P_{\mathrm{C} 2}=2\right.$ bar $)$ in presence of hydrogen $\left(P_{\mathrm{H} 2}=2\right.$ bar): Operating conditions and polymer properties

\begin{tabular}{ccccccccc}
\hline Run & $\begin{array}{c}\mathrm{T} \\
\left({ }^{\circ} \mathrm{C}\right)\end{array}$ & $\begin{array}{c}\mathrm{Cc}_{2} \\
\mathrm{~g} / \mathrm{L}\end{array}$ & $R_{p a}$ & $\begin{array}{c}\mathrm{Xc}_{1} \\
\%\end{array}$ & $\begin{array}{c}\mathrm{Xc}_{2} \\
\%\end{array}$ & $\begin{array}{c}\mathrm{Mw} \\
(\mathrm{kg} / \mathrm{mol})\end{array}$ & $\begin{array}{c}\mathrm{Mn} \\
(\mathrm{kg} / \mathrm{mol})\end{array}$ & $\begin{array}{c}\mathrm{PD}= \\
\mathrm{Mw} / \mathrm{Mn}\end{array}$ \\
\hline 11 & 50 & 2.11 & 1.3 & 60.9 & 64.2 & 241 & 31.1 & 7.7 \\
12 & 60 & 2.04 & 2.2 & 60.6 & 63.7 & 202 & 24.5 & 8.2 \\
13 & 75 & 1.94 & 3.5 & 65.8 & 70 & 191 & 24.8 & 7.7 \\
14 & 80 & 1.91 & 3.8 & 66.2 & 71.8 & 165 & 17.3 & 9.56 \\
15 & 90 & 1.86 & 2.2 & 68.4 & 71.4 & 126 & 10.7 & 11.7 \\
\hline
\end{tabular}

$R_{p a}$ is expressed as (kg PE/gcat.hr)

Comparing Table 4.2 and Table 4.3, we can state the following:

- the bulk concentration of ethylene in the gas-phase is only about $1 / 3$ of the bulk concentration in slurry

- the yield and its temperature-dependence is similar 
- in both cases, gas-phase and slurry, $\mathrm{Xc}_{2}>\mathrm{Xc}_{1}$, but the gas-phase crystallinity is lower in gas-phase polymerization and the difference between $1^{\text {st }}$ and $2^{\text {nd }}$ crystallinity is a little smaller in gas-phase

- the molecular weight is higher in the gas-phase with only one exception: at $90^{\circ} \mathrm{C}$

The question arises: why are the yields in the gas-phase and in slurry nearly identical despite monomer concentration in slurry being three times higher than that in gas-phase? A discussion of the rate profiles seems to be essential.

\subsubsection{Polymerization Rate Profiles}

The rate profiles of gas and slurry polymerizations under similar conditions differ significantly from each other, as is clear from comparing Figure 4.8 and Figure 4.17. However in both cases:

- the maximum polymerization rate is reached at $80^{\circ} \mathrm{C}$, and

- the polymerization rate at $90^{\circ} \mathrm{C}$ drops by about $40 \%$ compared to its maximum. A possible reason for such a difference in rate profiles was discussed in Chapter 3; see the section entitled "Moving from gas to slurry". As discussed previously for the second series, the rate decreasing behaviour at $90^{\circ} \mathrm{C}$ was attributed to high crystallinity and site deactivation by hydrogen. However, since there was no significant change in crystallinity of produced polymers in the third series (see Table 4.3), one can conclude that site deactivation by hydrogen at high temperature can be regarded as the cause of the observed rate decreasing.

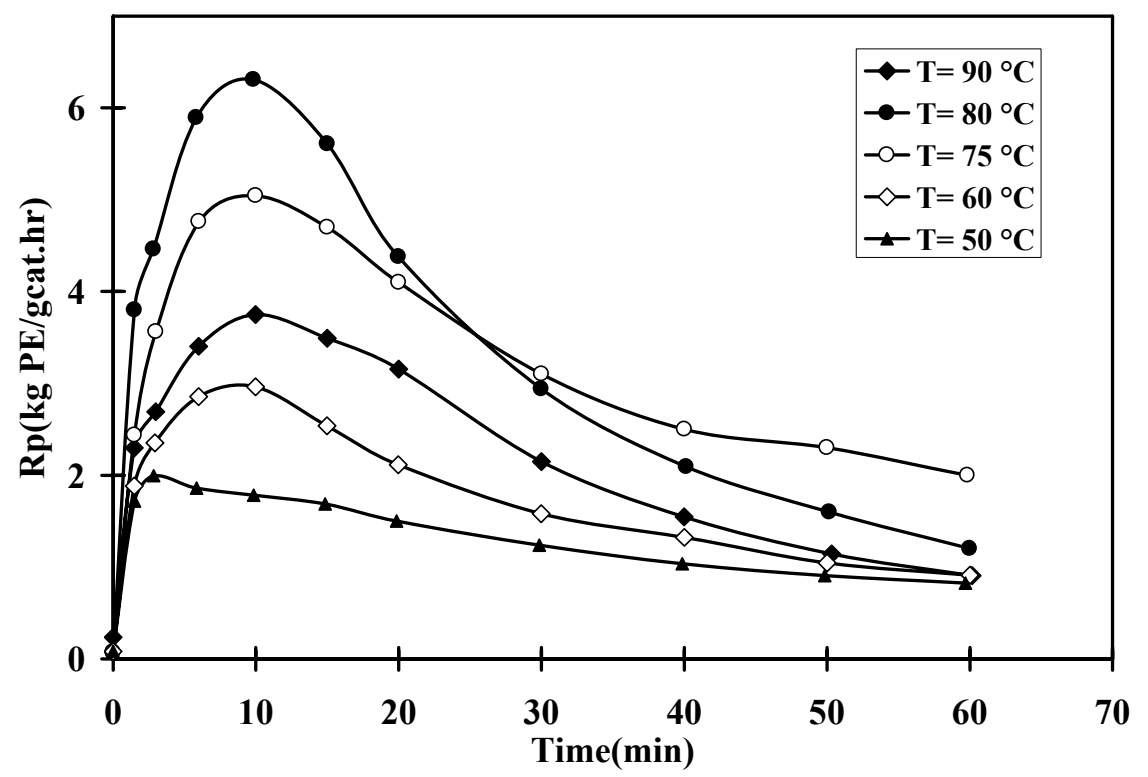

Figure 4.17- Gas-phase polymerization of ethylene $\left(P_{\mathrm{C} 2}=2\right.$ bar $)$ in presence of hydrogen $\left(P_{\mathrm{H} 2}=2\right.$ bar): rate profile

Comparison of yields at $80^{\circ} \mathrm{C}$ to $90^{\circ} \mathrm{C}$ for the second and third series extracted from Table 4.2 and Table 4.2 shows a roughly similar decrease in percentage (40\%). 
However, one should not overestimate this effect, because of the time-dependence of the yield ${ }^{1}$.

Comparing the rate profiles given in Figure 4.17 and Figure 4.8, one can see that despite the much lower ethylene bulk concentration - the polymerization rate in the starting phase increases much faster than in slurry, and as a result the maximum polymerization rate is reached much faster in gas-phase. This time, this effect is not caused by external fragmentation (see Figure 4.21), which does not mean that internal fragmentation does not contribute to the rate acceleration. We explain the quick initial rate acceleration in gasphase in terms of three effects:

1. gas diffusion is much faster than diffusion in hexane, i.e. the reaction starts more quickly

2. heat transfer particle-bulk in the gas-phase is more limiting than in slurry, i.e. the particles in the gas-phase suffer more easily from overheating [21], which means - at least initially - an accelerating polymerization rate.

3. the quick (internal) fragmentation process due to the high growth stress that clearly overcompensates for the countercurrent effect of the lower crystallinity/brittleness.

Still, the question remains open: to which extents are the active sites covered by polymer and how much does this contribute to a monomer concentration change near the active sites? If the active sites are completely covered by hexane-swollen (amorphous) polymer mixed with crystalline parts, then this must lead to a lower monomer concentration near the active sites[23,30], whereas the role of changing micro-porosity remains uncertain in gasphase polymerizations - it cannot be excluded that some micro-pores accelerate the monomer transport to the active sites, bypassing the diffusion barrier created by the semicrystalline PE. For all temperatures $>50^{\circ} \mathrm{C}$, the position of the peak remains constant at about $10 \mathrm{~min}$.

The largest difference between slurry and the gas-phase is the deactivation behaviour. In slurry, a nearly constant level is reached (see Figure 4.8) whereas in gasphase polymerization, quick deactivation follows directly after the rate maximum with a clear correlation between the height of the maximum and the deactivation constant.

In terms of the overall activation energy, $\mathrm{Kp} 0$ (the pre-exponential factor) and $\mathrm{Ea}, \mathrm{p}$ (the activation energy) of the gas-phase series were obtained by using equation (4.11) and the data shown in Table 4.3. We exclude the data for $90^{\circ} \mathrm{C}$. Figure 4.18 show the Arrhenius plot and the data obtained for $K p_{0}(5.21 \mathrm{e} 5)$ and $E_{a, p}(36.5 \mathrm{~kJ} / \mathrm{mol})$. Choi and Ray [75] have shown that the overall activation energy obtained in slurry propylene polymerization is significantly higher than those obtained for the gas-phase. We also found the same differences between gas-phase and slurry ethylene polymerization in the presence of hydrogen.

\footnotetext{
${ }^{1}$ The ratio of yields (gas phase polymerization / slurry) would drop down to low values if one continued polymerizing for 2 hours or more.
} 


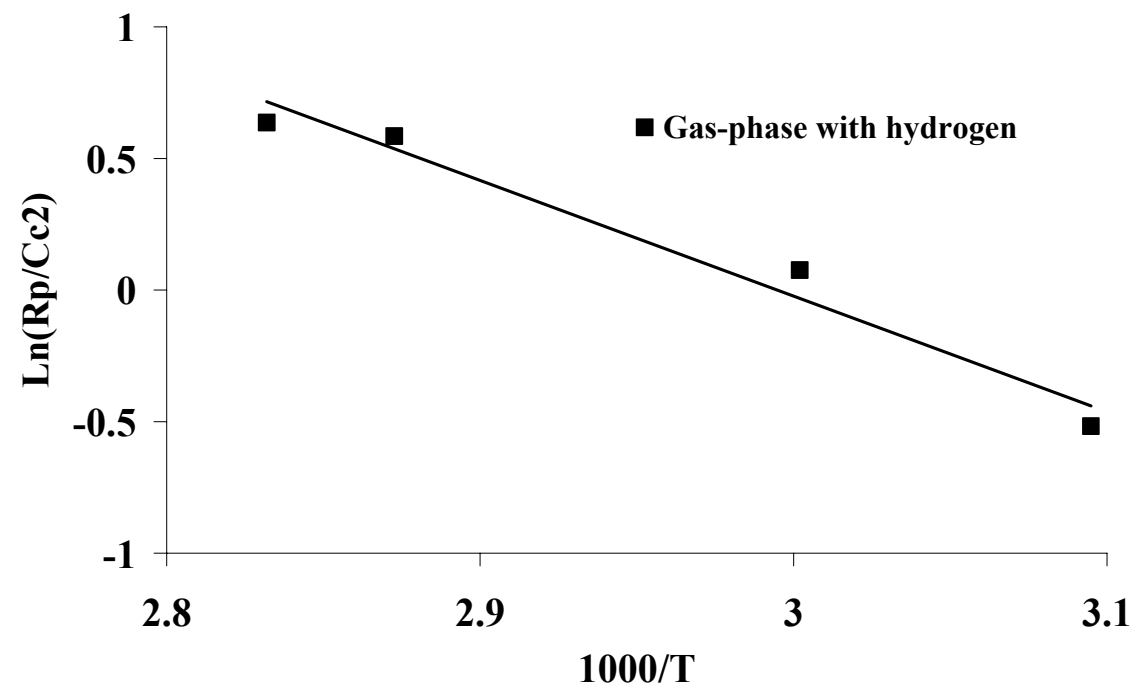

Figure 4.18-Arrhenius plot for estimating of $E_{a, p}$ based on the results shown Table 4.3 and obtained from equation (4.5) for the gas-phase series

\subsubsection{Molecular Weight and Crystallinity}

As for the other series, the MWD of polyethylene for the $3^{\text {rd }}$ series (see Figure 4.19 ) shifts leftwards towards a low molecular weight region as the operating temperature increases. Compared to the second series, the shoulder showing in the high molecular weight region is less noticeable. It seems that the hydrogen in the slurry phase increases the activity of the active centers responsible for producing high molecular more than it does for the gas-phase.

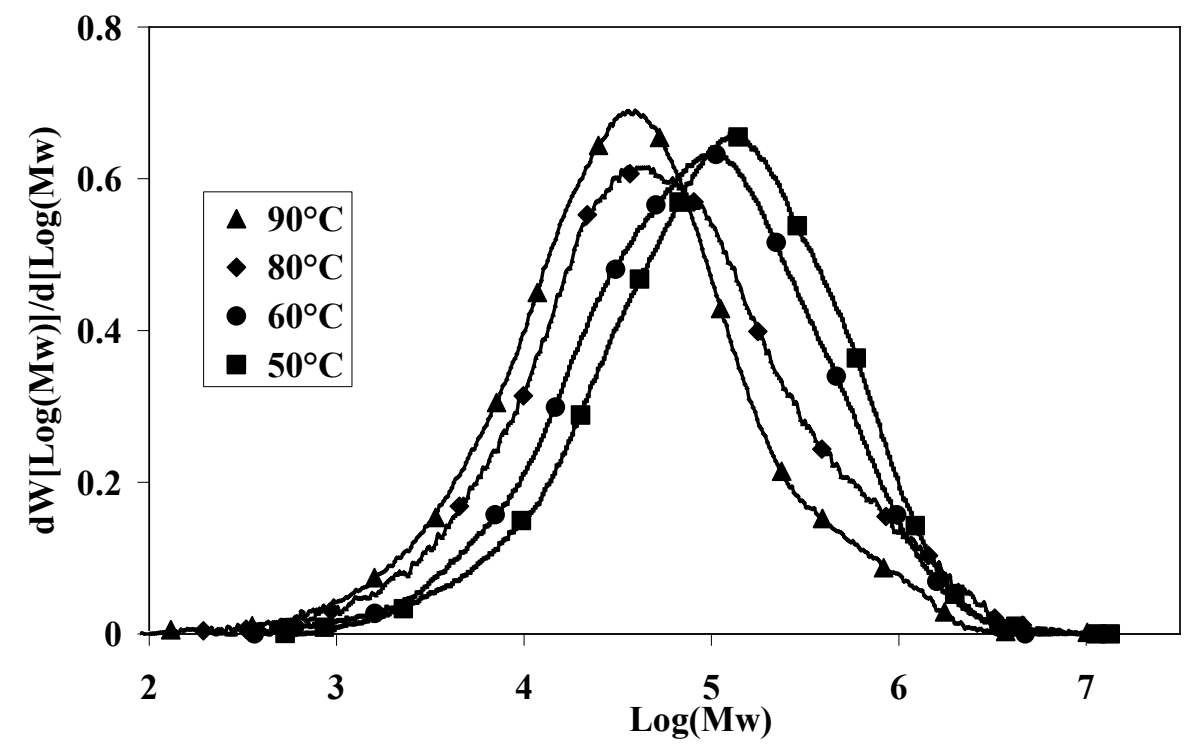

Figure 4.19-Temperature influence on MWDs of polyethylene produced in temperature series gas-phase ethylene polymerization with hydrogen $\left(\mathbf{P}_{\mathrm{C} 2}=2 \mathrm{bar} \& \mathbf{P}_{\mathrm{H} 2}=2 \mathrm{bar}\right)$ 
Figure 4.20 shows the DSC scan for $\mathrm{Xc}_{1}$ and $\mathrm{Xc}_{2}$. The crystallinity of the products from the gas-phase series at a given temperature is substantially lower than the corresponding crystallinity in the slurry phase. This demonstrates that the rate of crystal formation in slurry processes is higher than that in the gas-phase. This is a result of the lower micro-viscosity in the presence of hexane, but it does not explain the $\mathrm{Xc}_{2}$ difference between gas-phase and slurry products.

A)

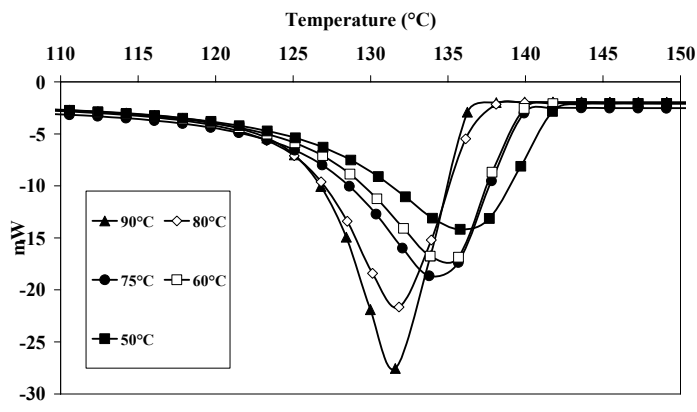

B)

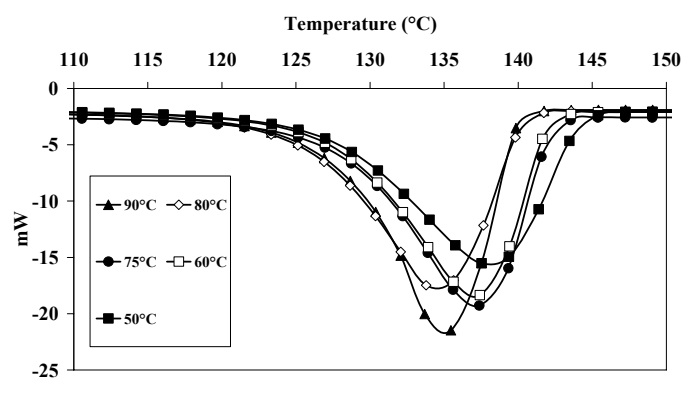

Figure 4.20-DSC scan for HDPE samples obtained in temperature series gas-phase ethylene polymerization with hydrogen (heating rate: $10 \mathrm{C} / \mathrm{min}$, N2 Atmosphere) (A): 1st heating (B): 2nd heating

\subsubsection{Morphology}

Figure 4.21 shows the effects of temperature on the cumulative particle size distribution of the third series normalized with the yield. The highest average normalized particle size occurs at $75^{\circ} \mathrm{C}$. It is also worth mentioning that the PSD profiles of polymer particles produced at $75^{\circ} \mathrm{C}$ in both the gas and slurry phases in the presence of hydrogen (Figure 4.12 and Figure 4.21) are almost identical.

From the morphological point of view, we recommend that the best operating temperature for producing less fines and having better spherical shape is $75^{\circ} \mathrm{C}$. This PSD profile also fit exactly with catalyst PSD profiles (Chapter 2) which is another reason that no fines and no agglomerate are produced at this temperature.

We found in the second series that whenever the polymer particles' crystallinity exceeds $70 \%\left(\mathrm{Xc}_{1}\right)$ or $73 \%\left(\mathrm{Xc}_{2}\right)$, a particle is more susceptible to breaking and fine production. From data obtained for the gas-phase polymerization in the presence of hydrogen (see Table 4.3), none of the runs exceeded this critical crystallinity. Therefore, we expected no significant change to the PSD profile of gas-phase series as the temperature increased (see Figure 4.21). This is in good agreement with the experimental findings. 


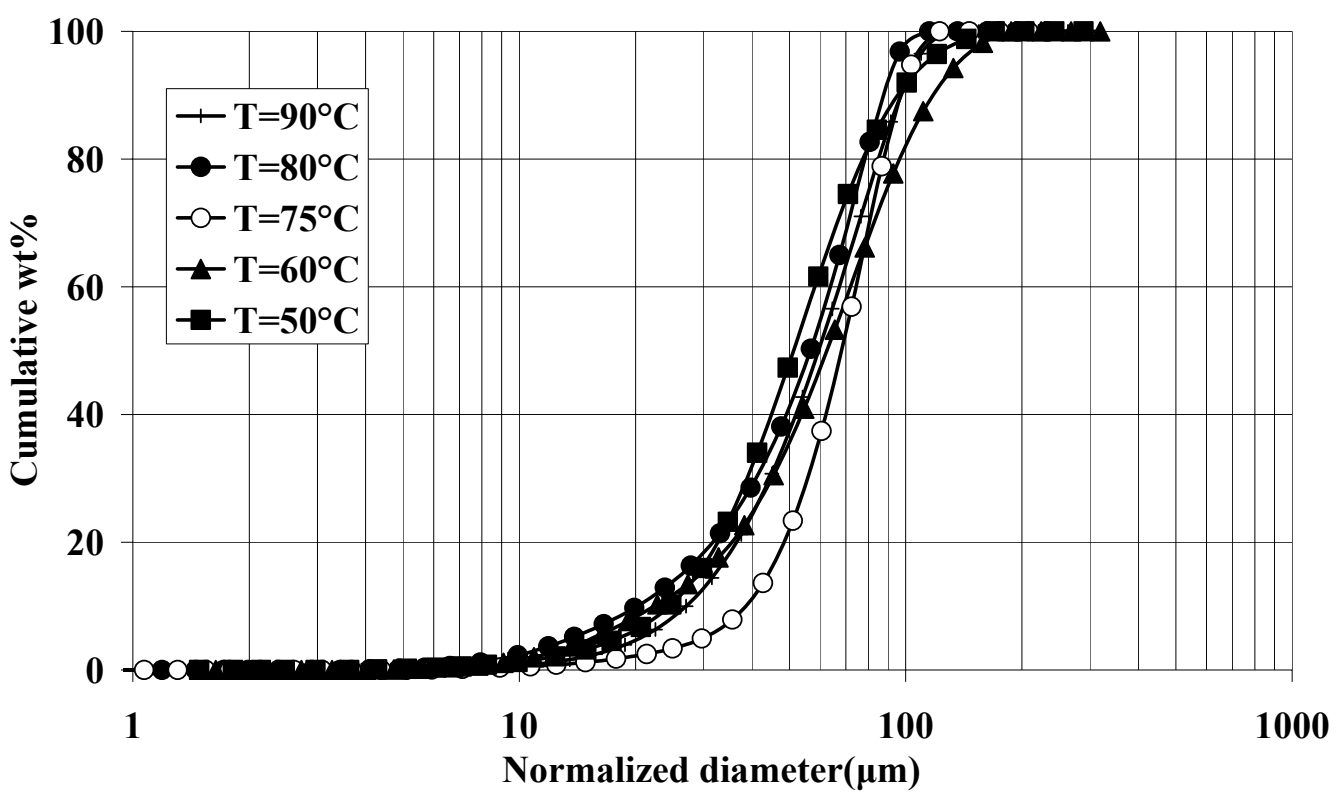

Figure 4.21-The influence of temperature on cumulative PSD profiles normalized with the yield $\left(\mathbf{P}_{\mathrm{C} 2}=\mathbf{2 b a r} \& \mathbf{P}_{\mathrm{H} 2}=2 \mathrm{bar}\right)$ in gas-phase homo-ethylene polymerization reaction with the presence of hydrogen

A)

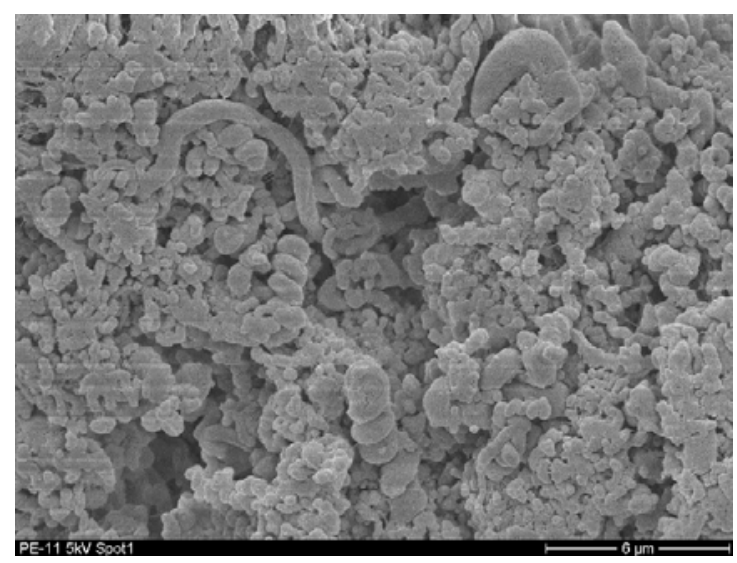

B)

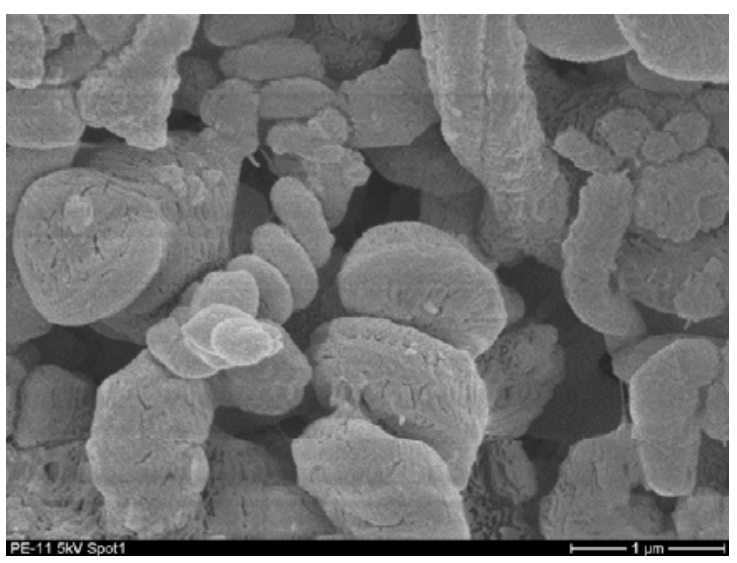

Figure 4.22-The SEM pictures of the polymer obtained at run 11: A) with $6 \mu \mathrm{m}$ contrast B) with 2 $\mu \mathrm{m}$ contrast

Figure 4.22 and Figure 4.23 show the scanning electron microscopy (SEM) images of polyethylene samples obtained in runs 11 and 15 of the gas-phase series respectively. The surface morphology of the polymer produced for run 11 was found to be a globular-like structure with some wormlike PE. However, the structure for run 15 seems to show a "cobweb" structure with low fibrillar structure. 
A)

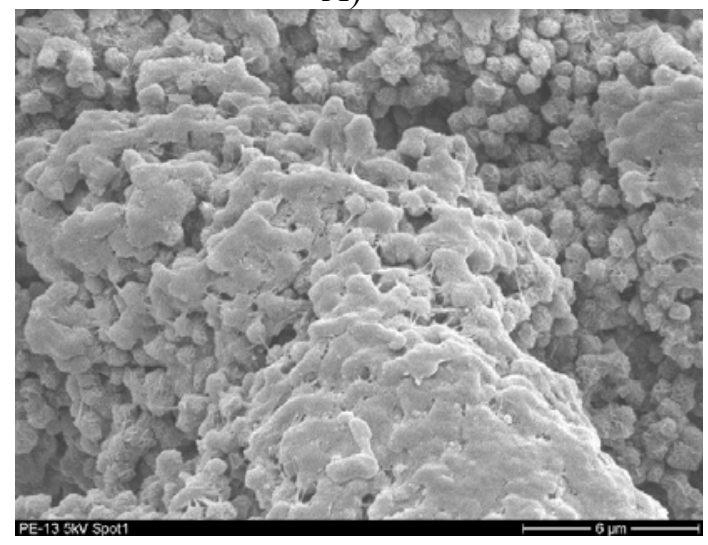

B)

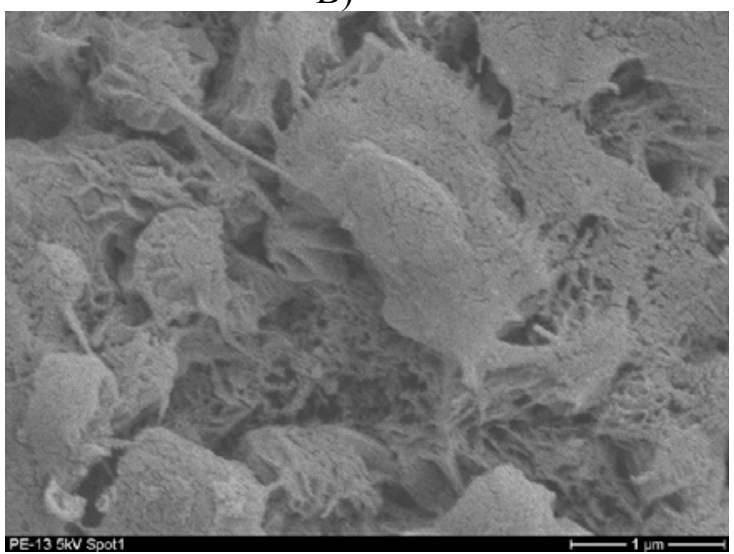

Figure 4.23-The SEM images of the polymer obtained during run 15: A) with $6 \mu \mathrm{m}$ contrast B) with $2 \mu \mathrm{m}$ contrast

Figure 4.24 shows the transmission electron microscopy (TEM) images of polyethylene samples obtained during runs 11 and 15 of the gas-phase series respectively. As can be seen, the size of lamella phase for the sample produced at the higher temperature (run 15 ) is greater. It means that the bigger crystals or larger domain of small crystals produced as the temperature of reaction increased. Again, this confirms that a higher chain mobility leads to larger crystals. This is a very important finding, because the larger the crystals is and near the critical crystallinity the more easily the fragmentation will proceed, with fines production in the worst case.
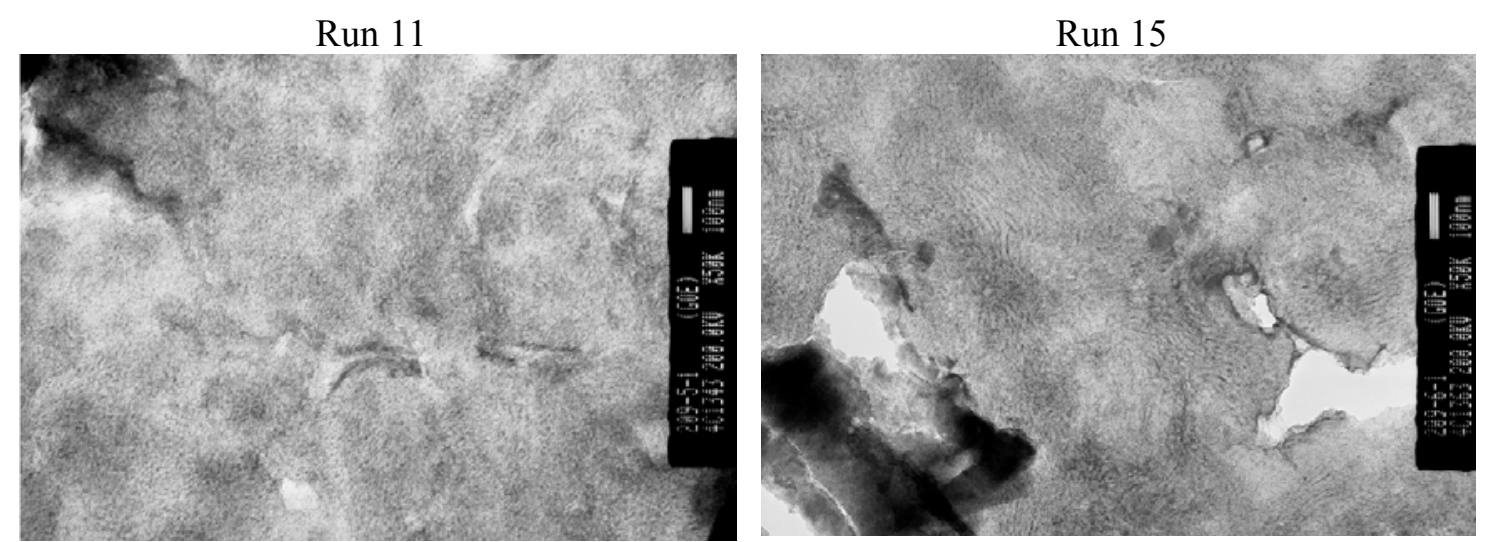

Figure 4.24-The TEM image of the polymer obtained during run 11 and 15

\subsection{Deconvolution analysis}

For a better understanding of the multi-center behaviors of heterogeneous ZieglerNatta catalyst used in this study and to compare this behavior in both slurry and gas-phase polymerization, deconvolution of MWD into Flory components has been done. Details of the deconvolution procedure were given in Chapter 2. 
Figure 4.25 compares two pairs of GPC curves deconvoluted to their Flory components for the polymer obtained at $60^{\circ} \mathrm{C}$ and $90^{\circ} \mathrm{C}$ performed in both slurry and gasphase ethylene polymerization. Except for deconvolution at $60^{\circ} \mathrm{C}$ in gas-phase condition, the five-site model can explain all the rest of GPC curved obtained at different temperature in both slurry and gas-phase reactions. As can be seen, at low temperature, the contribution of sites responsible for producing low molecular weight is lower when compared to those sites at high temperature.

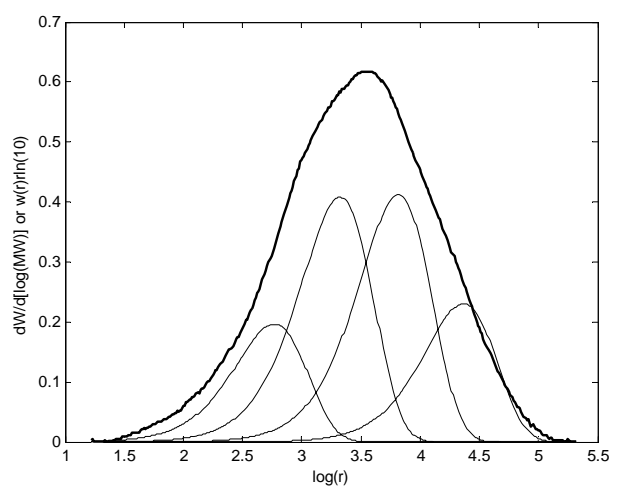

Gas-phase at $60^{\circ} \mathrm{C}$

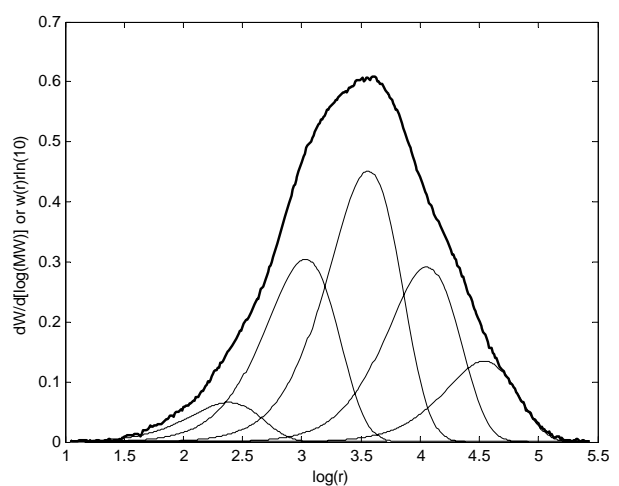

Slurry at $60^{\circ} \mathrm{C}$

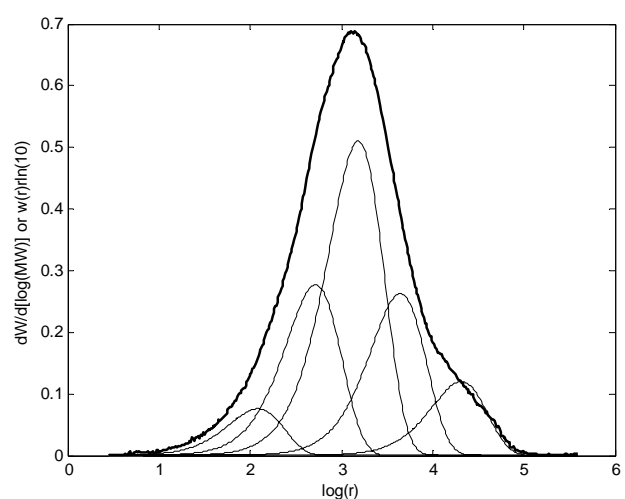

Gas-phase at $90^{\circ} \mathrm{C}$

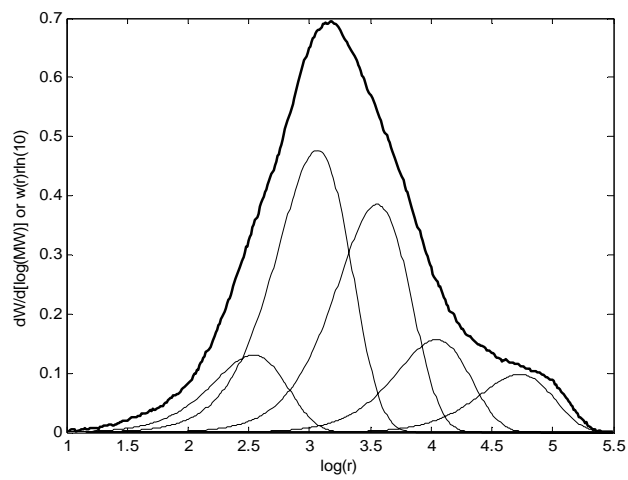

Slurry at $90^{\circ} \mathrm{C}$

Figure 4.25-Comparison of two pairs of deconvolution analysis at $60^{\circ} \mathrm{C}$ and $90^{\circ} \mathrm{C}$ performed in slurry and gas-phase ethylene polymerization $\left(P_{\mathrm{C} 2}=2\right.$ bar $\left.\& \mathbf{P}_{\mathrm{H}_{2}}=2 \mathrm{bar}\right)$

The detail of the calculated mass fraction of each component and its corresponding molecular weight at different temperatures in the slurry and gas-phase conditions are given in Table 4.4. From this table, it can be seen that the contribution of the first two sites increases by increasing the temperature but the opposite can be seen for the last two sites for both slurry and gas-phase conditions. In terms of the molecular weight of the Flory components, all sites' molecular weights increase as the temperature increases for slurry-made PE. This shows a meaningful discrepancy with the well-know theory that increasing temperature leads to higher activation energies for chain transfer than for propagation reactions resulting in lower molecular weights. It seems that in the slurry condition, the influence of temperature on 
changing the contribution of active centres on decreasing the molecular weight is more pronounced. Such an increase in sites' molecular weights cannot be observed in gas-phase condition as temperature increases.

Table 4.4-Temperature influence on parameters obtained by the deconvolution method in the

\begin{tabular}{cccccc}
\multicolumn{5}{c}{ presence of hydrogen } \\
\hline & & \multicolumn{2}{c}{ Slurry-made PE } & \multicolumn{2}{c}{ Gas-phase-made PE } \\
\cline { 2 - 6 } $\mathrm{T}\left({ }^{\circ} \mathrm{C}\right)$ & Site No & $\% m_{j}$ & $\mathrm{Mw}$ & $m_{j}$ & $\mathrm{Mw}$ \\
\hline $60^{\circ} \mathrm{C}$ & 1 & 5 & 3380 & 0 & - \\
& 2 & 25 & 15090 & 16 & 8210 \\
& 3 & 36 & 51170 & 33 & 29460 \\
& 4 & 23 & 159770 & 33 & 91750 \\
& 5 & 11 & 489910 & 18 & 325530 \\
\hline $80{ }^{\circ} \mathrm{C}$ & 1 & 10 & 4320 & 6 & 2450 \\
& 2 & 33 & 17470 & 29 & 11120 \\
& 3 & 31 & 53470 & 35 & 36090 \\
& 4 & 16 & 154890 & 19 & 116230 \\
& 5 & 10 & 622450 & 11 & 456280 \\
\hline $90{ }^{\circ} \mathrm{C}$ & 1 & 10 & 4830 & 6 & 1710 \\
& 2 & 38 & 16260 & 22 & 7290 \\
& 3 & 31 & 50340 & 41 & 21440 \\
& 4 & 13 & 155490 & 21 & 62090 \\
& 5 & 8 & 750390 & 11 & 288780 \\
\hline
\end{tabular}

\subsection{Summary}

In this chapter, we have discussed the influence of temperature between $50^{\circ} \mathrm{C}$ and $90^{\circ} \mathrm{C}$ at a constant ethylene pressure $\left(\mathrm{P}_{\mathrm{C} 2}=2\right.$ bar $)$ on polymerization rate profiles and polymer properties produced in both slurry and gas-phase ethylene polymerization using a ZieglerNatta catalyst co-catalyzed with TIBA, with $\left(\mathrm{P}_{\mathrm{H} 2}=2\right.$ bar) and without the presence of hydrogen.

In slurry polymerization without hydrogen, the catalyst activity increases over the whole range of temperature, whereas in the presence of hydrogen, in the gas-phase and slurry, the maximum polymerization rate was reached at $80^{\circ} \mathrm{C}$ although the activity at $90^{\circ} \mathrm{C}$ showed a polymerization rate drop of about $40 \%$ compared to the maximum. This can be explained by the competition of rate accelerating and rate decreasing factors, as described below. We concluded that the site deactivation by hydrogen (see equation 4.15 ) is more pronounced at higher temperature $\left(90^{\circ} \mathrm{C}\right)$.

We also confirmed the findings of Choi and Ray [75] that the overall activation energy for slurry ethylene polymerization in the presence of hydrogen is higher than that for the gas-phase. 
The molecular weight of polymers produced decreases with temperature and as a result of the addition of hydrogen. The higher $\mathrm{Mw}$ at $90^{\circ} \mathrm{C}$ compared to $80^{\circ} \mathrm{C}$ (slurry, without hydrogen) can be explained by a lower deactivation rate of high-molecular weight producing sites compared to low-molecular weight producing sites.

It is interesting to note that a clear increase in the high- Mw shoulders can be seen for all MWD produced in slurry in the presence of hydrogen as the temperature increases. This shoulder reveals that the Ziegler-Natta catalyst used in this study comprises different types of active centers, each showing a different temperature dependence. The active centers, which produce high molecular weight polymer, are less reactive to hydrogen at high temperature in slurry polymerization.

The polydispersity index (PD) increases significantly in the presence of hydrogen in slurry and gas-phase. Clearly, different active sites show different hydrogen responses, which leads to broadening of the MWD.

Deconvolution analysis demonstrates that a five-site model can generally explain all GPC curves obtained at different temperatures in both slurry and gas-phase polymerizations.

From the morphological point of view, for producing less fines and achieving better spherical shape, we recommended performing ethylene polymerization at $75^{\circ} \mathrm{C}$.

In all three experimental series, raising the temperature increases the crystallinity of produced polymers, which is in good agreement with lowering of the molecular weight. The increasing crystallinity is more pronounced in slurry ethylene polymerization in the presence of hydrogen. It demonstrates that the rate of crystallization process is faster in slurry compared to gas-phase. Furthermore, without hydrogen, the first crystallinity is higher than the second one. The order is reversed when hydrogen is present during the reaction.

In terms of particle size and particle size distribution, raising the temperature leads to changed behavior in three mentioned series. In the first series (slurry without hydrogen), no significant changes in PSD profiles were seen as the temperature changes. This shows that the growth and thermal stresses due to the temperature rising do not break the growing catalystpolymer particle. When hydrogen was introduced in the second series, significant fines formation occurred at higher temperature. We attributed this behavior to the high crystallinity obtained. We observed that whenever the measured crystallinity rose above $70 \%$ for 1 st run or higher than $75 \%$ for 2 nd run, the powders would have many fines.

\subsection{Conclusions for Process Modeling}

The influence of temperature is clearly more complex then is often interpreted. With increasing temperature (within the range of parameters studied) the following important parameters and processes were found to change:

1. all kinetic constants are increasing; the transfer reactions increase more rapidly due to their higher activation energy

2. in slurry, the vapour pressure of hexane increases exponentially causing a higher solubility of hexane in the amorphous polyethylene; this changes all equilibrium 
and transport properties within the particles, especially the solid phase viscosity and the diffusivity of all components (monomer, hydrogen, co-catalyst, polymer)

3. the higher mobility of freshly produced polymers within a matrix, the viscosity of which is lowered, leads to faster crystallization; ; subsequently, more and larger crystals increase the brittleness of the particle; this promotes the fragmentation that can lead - in an extreme case to shifting the normalized PSD to the left so that fines are generated; fragmentation, as a physical effect, generates new active sites, which leads to a faster chemical reaction

4. in gas-phase polymerizations, the overheating of particles must be taken into account; especially at the beginning of the polymerization process, higher thermal and mechanical stresses are caused while the polymerization rate accelerates quickly; certainly, the fragmentation follows this course, but does not lead to external fragmentation - no fines are generated at higher temperatures due to the higher stickiness of the amorphous PE; fast deactivation during the later stage in gas-phase can be caused by:

- decreasing co-catalyst concentration around the active sites, the reason for which can be seen in the polymer flow from active sites to particle surface

- $\quad$ thermal deactivation of some active sites during the overheating

Neither effects is present in slurry, because of the excellent heat transfer particle-bulk and because of the solubility of the co-catalyst in hexane accompanied by the viscosity-decreasing hexane solubility in the amorphous polyethylene. 



\section{Chapter 5}

\section{Influence of Ethylene Pressure}

\subsection{Introduction}

For almost all Ziegler-Natta catalysts, ethylene shows the highest reactivity compared to other olefins. Kissin reported in 1999 [76] that ethylene reactivity is three to four times higher than the reactivity of polypropylene and over 50 times higher than that of 1-hexene or 4-methyle-1-pentene. Productivity, product quality, costs and the safety of industrial polymerization processes depend strongly on ethylene pressure.

Most researchers simplify the polymerization rate as follows:

$$
R_{p} \approx k_{p} C_{C 2}^{n} \approx k_{p} P_{C 2}^{n} \quad \text { Equation } 5.1
$$

where, $C_{C 2}$ is the bulk concentration of ethylene, $P_{C 2}$ is ethylene pressure and $n$ is the order of reaction related to ethylene. Depending on the type of catalyst and the conditions around the active sites, $n$ has been found to vary between 1 and $2[18,22,38,45,76-80]$.

The influence of ethylene pressure in homo ethylene polymerization was mainly investigated as follows:

- varying the ethylene pressure in absence of hydrogen

- varying the ethylene pressure under a constant hydrogen pressure

- varying the ethylene pressure with constant hydrogen: ethylene ratio

In 1998, Kissin [18] performed series of gas-phase ethylene polymerizations with and without hydrogen in an ethylen pressure range of $0.2-1.4$ bar. He reported $n=1.5 \ldots 1.6$.

In 1991, D. Lynch and S. Wanke [80] found $n=1$ in gas-phase ethylene polymerization in the pressure range of 0.1 to 0.8 bar within a temperature range of 20 to $90{ }^{\circ} \mathrm{C}$ and in absence of hydrogen.

In 1999, Kissin et al. [76] carried out slurry (in n-heptane) and gas-phase ethylene polymerizations by varying the ethylene pressure between 0.3 and 1.3 bar without hydrogen and using various types of Ziegler-Natta catalyst. In both the phases, gas and slurry phases, they found $n=1.6 \ldots 2$. Additionally, they showed that their four Flory components - with pressure-dependent contributions - explain the multicenter behaviour of the catalysts they used.

In 2005, Bergstra et al. [45] found n (depending on ethylene pressure) to be between 1 and 2 for both slurry and gas-phase ethylene polymerization when using a metallocene catalyst. By increasing ethylene pressure (up to 40 bar!), n changed from 2 to 1 . The authors explained this effect by the complex formation expressed in reaction 5.1: 


$$
\mathrm{C}^{0}+\mathrm{M} \underset{\mathrm{k}_{2}}{\stackrel{\mathrm{k}_{1}}{\rightleftharpoons}} \mathrm{C}^{\mathrm{M}}
$$

Reaction 5.1

where $\mathrm{C}^{0}$ is a uncomplexed active centre, $\mathrm{C}^{\mathrm{M}}$ is a complexed active centre with ethylene (M). This leads to a rate expression that explains their findings:

$$
R_{p}=k_{p} \cdot C_{t} \cdot \frac{K_{A} \cdot M^{2}}{1+K_{A} \cdot M}
$$

where $\mathrm{C}_{\mathrm{t}}$ is the total concentration of active centers and $\mathrm{K}_{\mathrm{A}}$ is the equilibrium constant of complexation $k_{1} / k_{2}$. At a low ethylene pressure (low $M$ ), it holds that $K_{A} \cdot M<<1$ and the reaction rate is second order with respect to ethylene concentration M:

$$
R_{p}=k_{p} \cdot K_{A} \cdot C_{t} \cdot M^{2} \quad \text { Equation } 5.3
$$

However, when the ethylene concentration is high, $\mathrm{K}_{\mathrm{A}} \cdot \mathrm{M}>>1$, the reaction rate is first order:

$$
R_{p}=k_{p} \cdot C_{t} \cdot M \quad \text { Equation } 5.4
$$

Based on the model of Bergstra et al, the order of reaction strongly depends on the range of ethylene pressure studied.

In this thesis, the quantitative description of the polymerization rate is not just of academic interest in terms of kinetics - it is clear from equation 5.1 that the growth stress is determined by the ethylene pressure which has a significant impact on fragmentation, fines generation, new sites generation, activation, deactivation, molecular weight and crystallinity (see the "basic hypothesis" in Chapter 3). Due to the absence of a liquid phase in gas-phase polymerization, one can expect a different impact of the monomer pressure on all the items mentioned. This will be investigated quantitatively in this chapter.

Three series of experiments were performed to study the influence of the ethylene pressure on the process behaviour:

Series 1: in slurry in the absence of hydrogen

Series 2: in gas-phase in the presence of hydrogen

Series 3 : in slurry at a constant hydrogen: ethylene pressure ratio, $\mathrm{P}_{\mathrm{H} 2} / \mathrm{P}_{\mathrm{C} 2}$

\subsection{Slurry polymerization in absence of hydrogen}

Two reproducible slurry ethylene polymerizations were executed at different ethylene pressures by keeping all other variables constant. The reactor and polymerization procedures used were described in Chapter 2. Table 5.1 lists the operating conditions and the recipe for the catalyst preparation.

Table 5.2 compares the properties of obtained polymer obtained. 
Table 5.1-the operating conditions and recipe for the catalyst preparation

\begin{tabular}{cccccccc}
\hline $\begin{array}{l}\text { Hexane } \\
(\mathrm{mL})\end{array}$ & $\begin{array}{c}\mathrm{T} \\
\left({ }^{\circ} \mathrm{C}\right)\end{array}$ & $\begin{array}{c}\mathrm{P}_{\mathrm{C} 2} \\
(\mathrm{bar})\end{array}$ & $\begin{array}{c}\mathrm{C}_{\mathrm{C} 2} \\
(\mathrm{~g} / \mathrm{L})\end{array}$ & $\begin{array}{c}\text { Catalyst } \\
(\mathrm{mg})\end{array}$ & $\begin{array}{c}\text { Pre-contacting } \\
\text { time } \\
(\mathrm{min})\end{array}$ & $\begin{array}{c}\text { Co-catalyst TIBA } \\
(\mathrm{mg})\end{array}$ & $\begin{array}{c}\text { Scavenger } \\
\text { TIBA }(\mathrm{mg})\end{array}$ \\
\hline 700 & 80 & 2 & 4.8 & 20 & 30 & 200 & 200 \\
700 & 80 & 6 & 15.3 & 20 & 30 & 200 & 200 \\
\hline
\end{tabular}

Table 5.2-Comparison of the properties of polymer obtained in the slurry ethylene series

\begin{tabular}{cccccccc}
\multicolumn{8}{c}{ polymerization in the absence of hydrogen } \\
\hline Run & $\begin{array}{c}\mathrm{R}_{\mathrm{P}}{ }^{*} \\
\text { average }\end{array}$ & $\begin{array}{c}\mathrm{M}_{\mathrm{w}} \\
(\mathrm{kg} / \mathrm{mol})\end{array}$ & $\begin{array}{c}\mathrm{M}_{\mathrm{n}} \\
(\mathrm{kg} / \mathrm{mol})\end{array}$ & $\mathrm{M}_{\mathrm{w}} / \mathrm{M}_{\mathrm{n}}$ & $\begin{array}{c}\mathrm{X}_{\mathrm{C} 1} \\
\%\end{array}$ & $\begin{array}{c}\mathrm{X}_{\mathrm{C} 2} \\
\%\end{array}$ & $\begin{array}{c}\mathrm{Tm} \\
\left({ }^{\circ} \mathrm{C}\right)\end{array}$ \\
\hline $1-\mathrm{P}_{\mathrm{C} 2}=2$ bar & 2.8 & 560 & 114 & 4.9 & 67 & 60 & 136 \\
$2-\mathrm{P}_{\mathrm{C} 2}=6$ bar & 10.8 & 815 & 185 & 4.4 & 65 & 56 & 137 \\
\hline
\end{tabular}

$* \mathrm{R}_{\mathrm{p}}$ in $\mathrm{kg} \mathrm{PE} /$ gcat.hr

Figure 5.1 shows the rate-profiles for mentioned experiments. The curves show typical "build-up" profiles without any unusual catalyst decay. We explain this in terms of a sufficiently good back-diffusion of the co-catalyst and - it seems - there is no stage during the lifetime of active sites that allows deactivation.

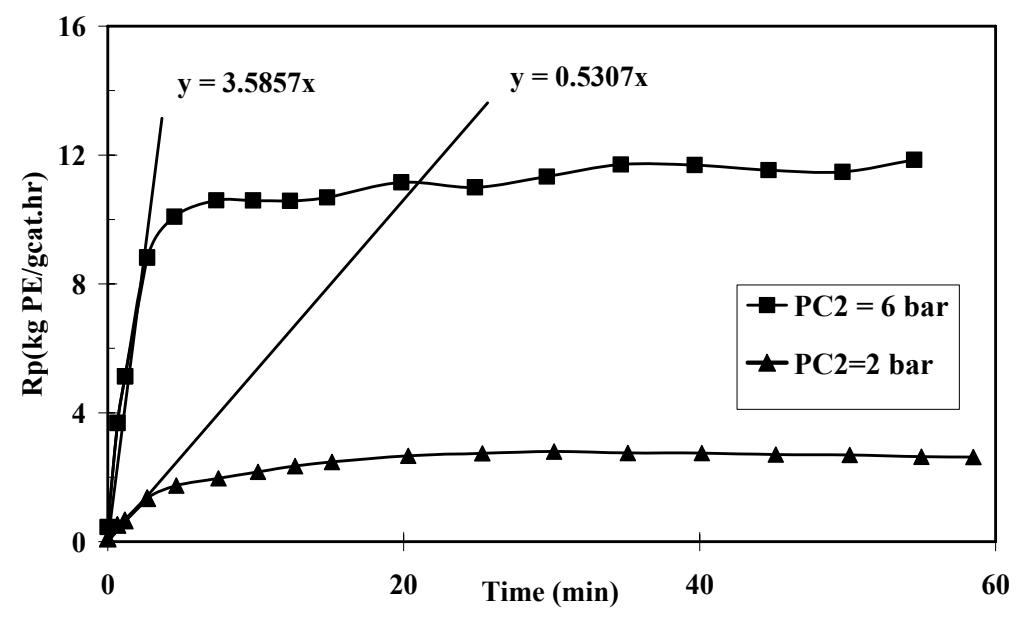

Figure 5.1-Effect of ethylene pressure on the reaction rate-profile

The plateau activity changes from 2.8 to 10.8 (factor 3.8 ) by increasing the pressure by a factor of 3 - this comes close to a first order influence on the monomer pressure. Let us use equation 5.2 to describe these plateau activities as function of pressure as follows: We rewrite equation 5.2 using the monomer pressure $\mathrm{P}$ instead of concentration, and then we substitute constant parameters into one constant: 


$$
R_{p}=\frac{K_{3} \cdot P^{2}}{1+K_{4} \cdot P}
$$

We define the ratio of the two measurements carried out at different pressures $\mathrm{P}_{1}$ and $\mathrm{P}_{2}$ :

$$
X=\frac{R_{p, 1}}{R_{p, 2}}=\frac{P_{1}^{2}\left(1+K_{4} \cdot P_{2}\right)}{P_{2}^{2}\left(1+K_{4} \cdot P_{1}\right)}
$$

Now, the constant $\mathrm{K}_{4}$ can be estimated from this ratio $\mathrm{X}$ and we obtain $\mathrm{K}_{4}=1$. Based on just these two measurements, we could conclude from the denominator of equation 5.5 that a $2^{\text {nd }}$ order- $1^{\text {st }}$ order model with increasing pressure is useful. But is this true? We will discuss this below.

The initial slopes of the rate curves shown in Figure 5.1 varies more than one might expect from the pressure ratio: the ratio of the slopes is about 6.7, which is much higher than 3 (the pressure ratio). This can be explained as follows: the higher polymerization rate at 6 bar leads to faster fragmentation and consequently faster generation of new active sites - these new sites accelerate the new site generation until the maximum is reached. Obviously, during the initial polymerization, the pressure dependency depends on fragmentation.

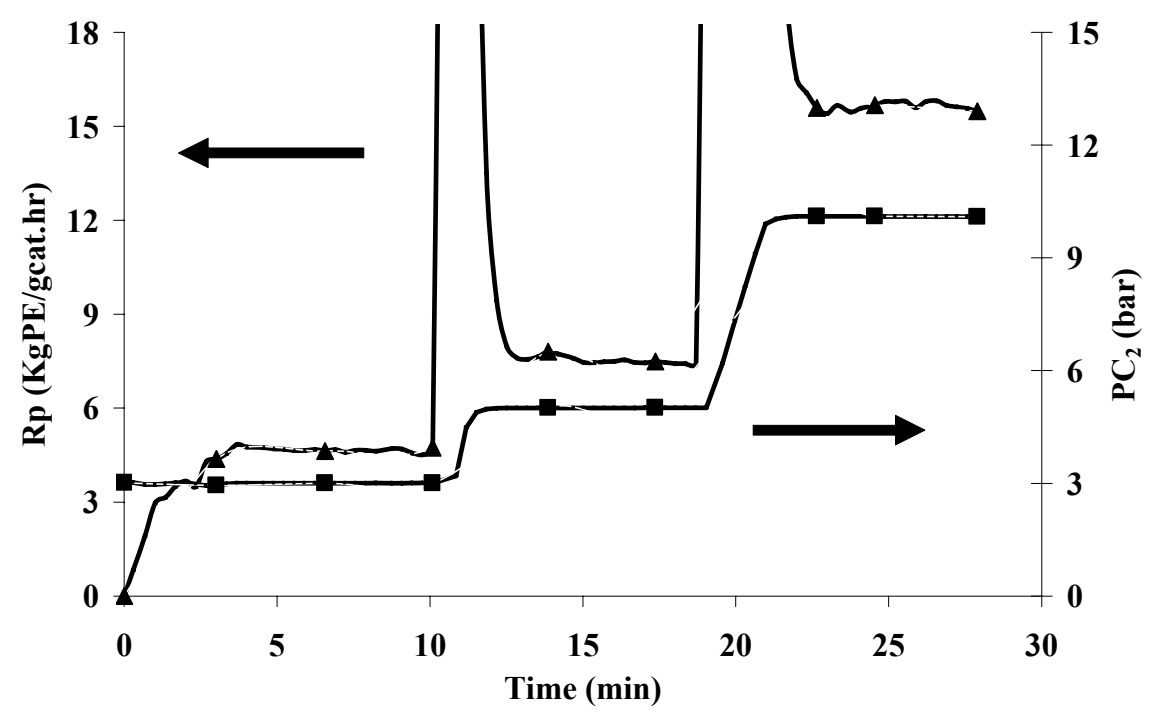

Figure 5.2-Effect of stepwise increase of ethylene pressure on the reaction rate-profile of slurry ethylene polymerization at $\mathrm{T}=80^{\circ} \mathrm{C}$ in the absence of hydrogen $\triangle R p, P_{C 2}$

For neglectible catalyst decay after the initial phase, a stepwise increase in pressure of ethylene in one isothermal experiment can be executed in order to quantify the pressure dependence - which is then not influenced by the initial fragmentation and new sites generation. Other researchers $[18,22,38,81]$ also implemented this technique as a so-called "perturbation of monomer pressure". Figure 5.2 shows the resulting rate-profiles.

As can be seen, stepwise increasing of the ethylene pressure from 3 to 10 bars increases the corresponding reaction rate in proportion to the pressure up to 10 bars. Plotting 
the rate of polymerization in the constant region versus ethylene pressure for all data measured (including the results shown in Figure 5.1) leads to Figure 5.3. The conclusion is clear: without the influence of the catalyst fragmentation during the initial stage, this catalyst shows a linear pressure dependence. Finally, after fragmentation, Figure 5.3 gives us a good reason to use the first order pressure dependency in our model.

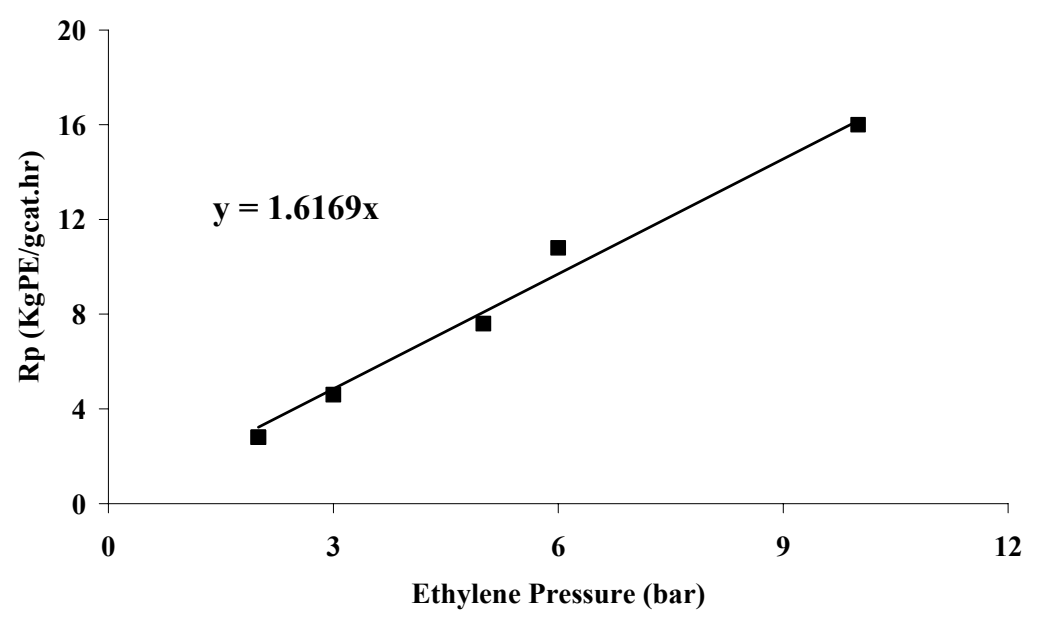

Figure 5.3-plot for calculating the reaction order with respect to ethylene pressure

Table 5.2 reveals that as the ethylene pressure increases, the weight and number average molecular weight increases. This can also be seen in Figure 5.4, which shows MWDs of the produced polymer.

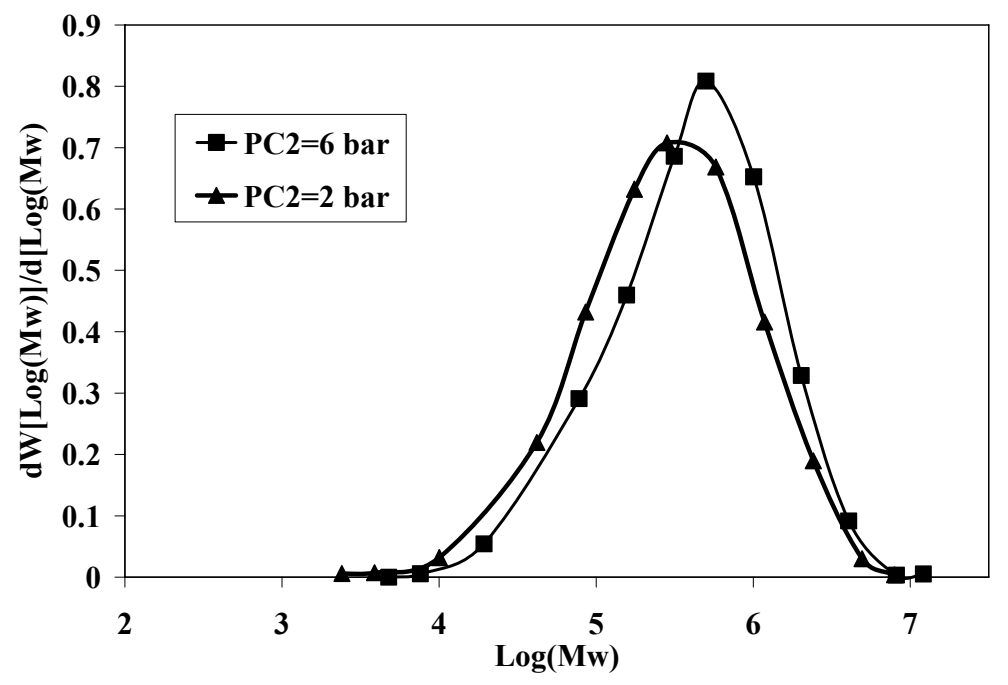

Figure 5.4-MWDs of the produced polymer

A possible explanation is given by equation 5.7 which is valid for every site of this multi-site catalyst 


$$
\frac{1}{M_{n}} \approx \frac{k_{t M}}{k_{p}}+\frac{k_{t A}[A]}{k_{p}[M]}+\frac{k_{t S}}{k_{p}[M]}+\ldots \ldots . . \quad \quad \text { Equation } 5.7
$$

The second and third terms of the right-hand side of equation 5.7, decreases with increasing monomer pressure, and a higher molecular weight results at higher monomer pressures.

Additionally, it is possible that the parameters in equation 5.7 will be different for different active site types, and the contribution of different Flory components can depend on monomer pressure. To investigate this assumption, we applied the deconvolution analysis as described in Chapter 2. The results are shown in Table 5.3 and Figure 5.5. At higher ethylene pressures, centre I, which produces the lowest molecular weight, is not active at all. Furthermore, the contribution of the second one (II) is also lower for higher ethylene pressure. This leads to an additional increase in $\mathrm{Mw}$ at higher pressures.

Table 5.3- Ethylene pressure influence on Flory parameters in slurry conditions

\begin{tabular}{ccccccc}
\hline $\mathrm{P}_{\mathrm{C} 2}$ (bar) & & Centre I & Centre II & Centre III & Centre IV & Centre V \\
\hline \multirow{2}{*}{2} & $\mathrm{Mw} / 10,000$ & 1.40 & 5.43 & 1.71 & 41.8 & 82.0 \\
& Mass\% & 3 & 22 & 41 & 20 & 13 \\
6 & $\mathrm{Mw} / 10,000$ & - & 5.69 & 20.9 & 49.1 & 113 \\
& Mass\% & - & 14 & 38 & 36 & 13 \\
\hline
\end{tabular}

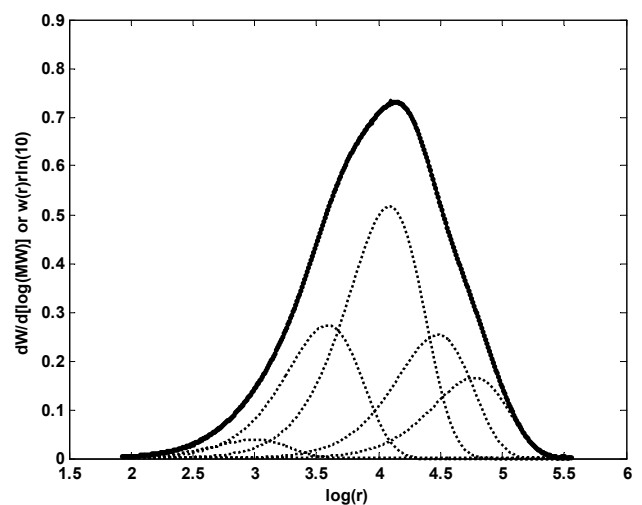

$\mathrm{PC}_{2}=2$ bar

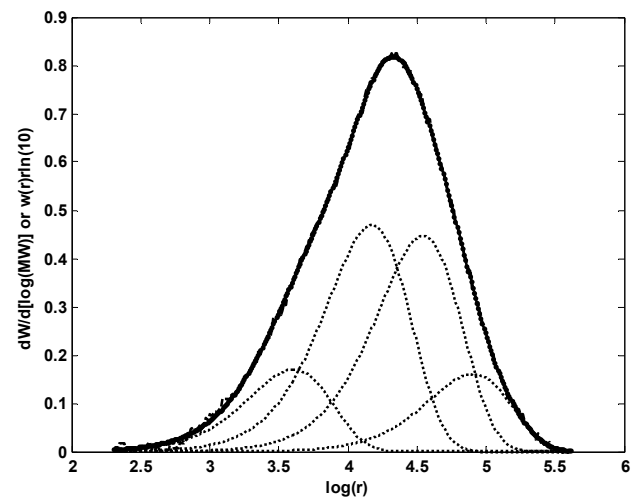

$\mathrm{PC}_{2}=6$ bar

Figure 5.5- Deconvolution analysis of polyethylene produced at different ethylene pressures, see Table 5.1

Is there a pressure influence on the PSD? Does the higher growth stress [66] at higher pressure lead to particle disintegration and fines generation? Or is the strength of the skin around the particles [82] sufficiently high to keep the fragments together without disintegration under these conditions?

Figure 5.6 shows the effect of ethylene pressure on cumulative particle size distribution normalized with the yield of the polymer extracted from the two experiments as described in Table 5.1. There is a small shift of the curve to the left towards small particles as ethylene pressure increases from 2 bars to 6 bars. The shift is more pronounced for bigger 
particles than for smaller particles. Therefore, one might expect that the relaxation processes for releasing the growth stress without any rupture of skin can eassily take place for small particles. Big particles - under the same conditions - accumulate a higher stress and can more easily disintegrate.

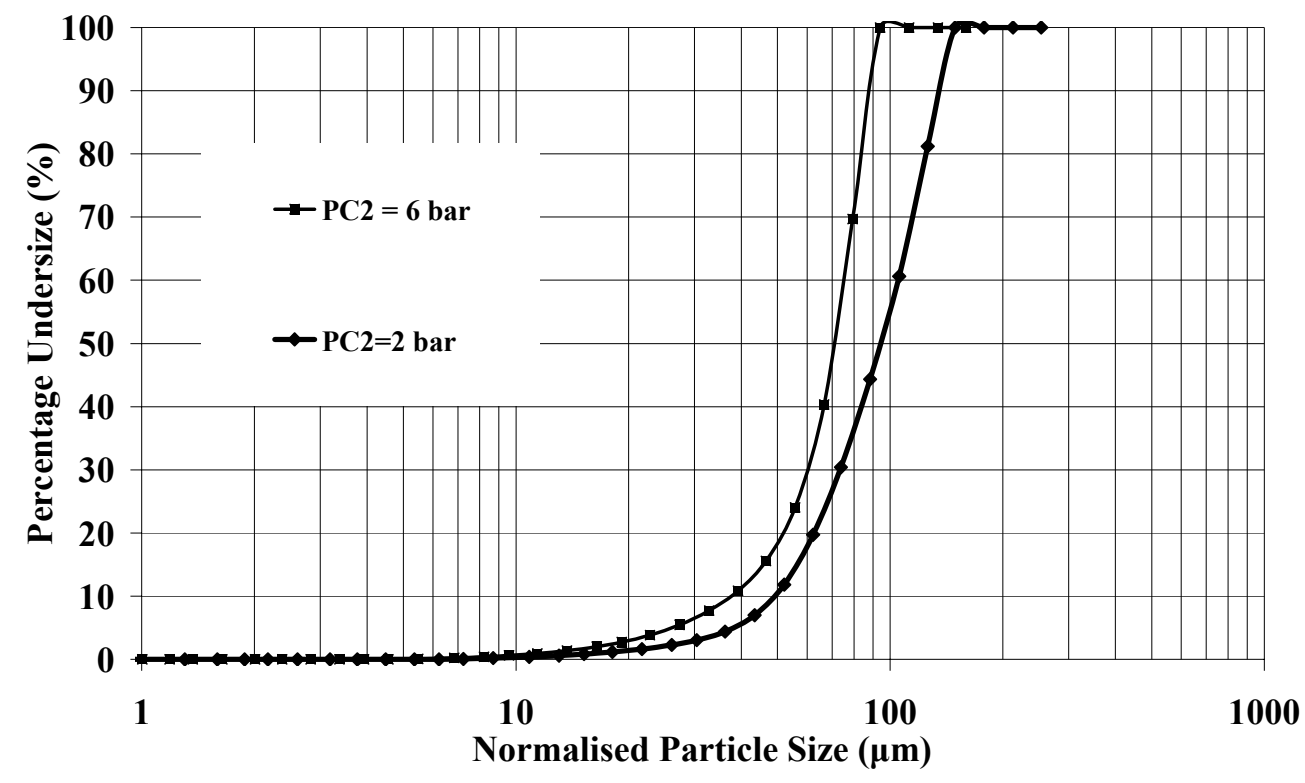

Figure 5.6-Effect of ethylene pressure on PSD

\subsection{Gas phase polymerization at constant hydrogen pressure}

Four gas-phase experiments were performed at ethylene pressures between 2 and 5 bar while keeping all other parameters constant. The same experimental set-up, experimental procedure for gas-phase reaction, chemicals and catalyst system, as explained in Chapter 2, were used in these series. The catalyst system was $\mathrm{C}_{\mathrm{g}}$ (gas-phase catalyst) and TIBA was used as the scavenger and co-catalyst. Table 5.4 lists the common conditions for all four experiments.

Table 5.4- Common operating conditions for gas-phase ethylene polymerizations

\begin{tabular}{cccccccc}
\hline Run & Media & $\begin{array}{c}\mathrm{T} \\
\left({ }^{\circ} \mathrm{C}\right)\end{array}$ & $\begin{array}{c}\mathrm{P}_{\mathrm{H} 2} \\
(\mathrm{bar})\end{array}$ & $\begin{array}{c}\text { Catalyst } \\
(\mathrm{mg})\end{array}$ & $\begin{array}{c}\text { Pre-contacting } \\
\text { time }(\mathrm{min})\end{array}$ & $\begin{array}{c}\text { Co-catalyst } \\
\text { TIBA }(\mathrm{mg})\end{array}$ & $\begin{array}{c}\text { Scavenger } \\
\text { TIBA(mg) }\end{array}$ \\
\hline \multirow{2}{*}{ all } & $\begin{array}{c}110 \mathrm{mg} \\
\text { salt }\end{array}$ & 75 & 2 & 20 & 30 & 200 & 200 \\
\hline
\end{tabular}

Figure 5.7 shows the reaction rate profiles. The higher the monomer pressure, the higher the peak activity and therefore the faster the catalyst decays. 


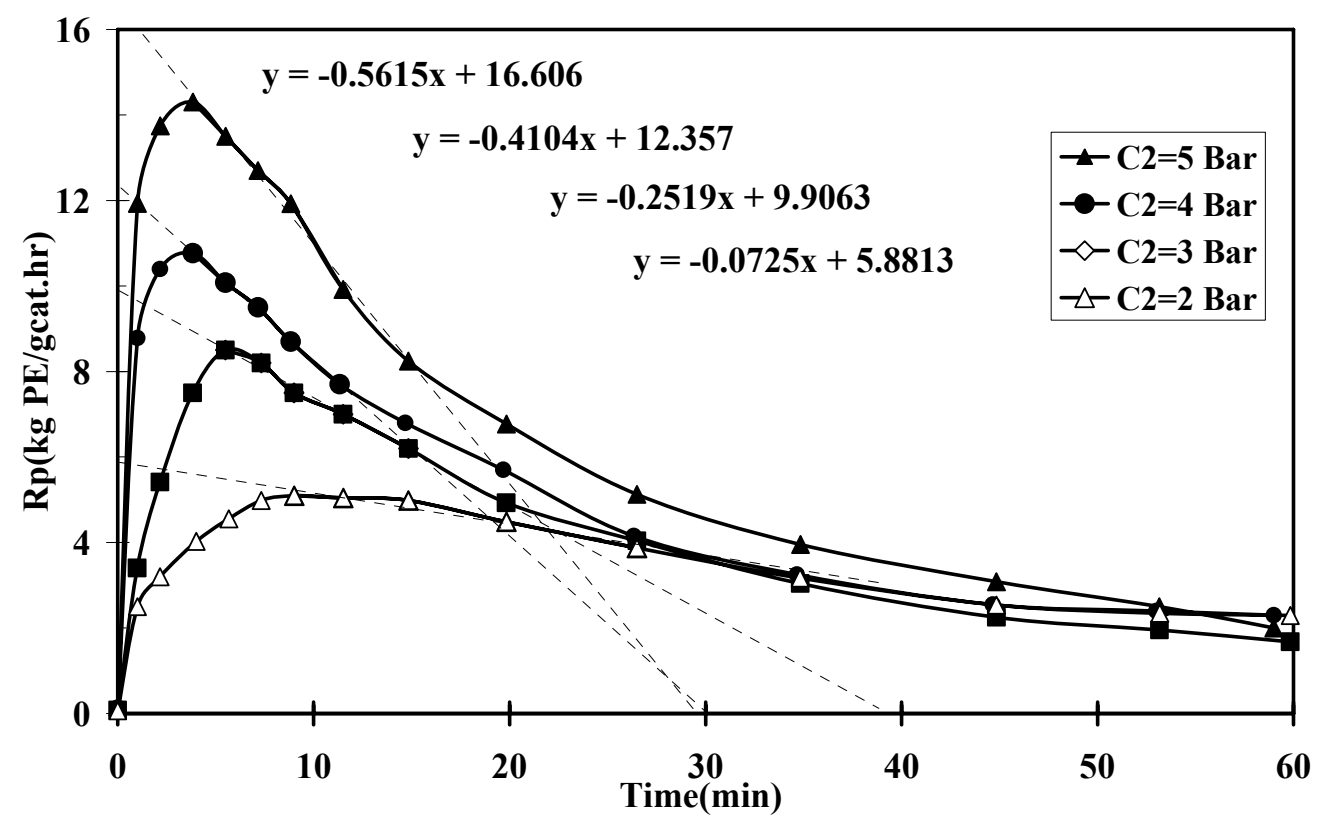

Figure 5.7- Effect of ethylene pressure on the reaction rate-profile of gas-phase ethylene polymerization at $\mathrm{T}=75^{\circ} \mathrm{C}$, and the plot of the relevant decay line

The slope of decay is extracted from Figure 5.7 and shown in Figure 5.8. It is a linear function of the ethylene pressure. The higher decay at higher polymerization rates can be attributed to different causes. One cause could be the overheating during the initial phase, but it is unclear why this would lead to a linear decrease in catalyst deactivation.

Another more reasonable explanation is the mass transfer behaviour of the cocatalyst. Assuming the co-catalyst is in equilibrium with the $\mathrm{Ti}$, a certain portion of this nearto-active-site co-catalyst can be removed from the active sites by the freshly produced polymer. This creates a concentration difference, which in turn forces the co-catalyst backdiffusion against the convective polymer flow. At higher polymerization rates, this must lead to increased "deactivation" as a consequence of the decreasing co-catalyst concentration near the active site.

A third - most reasonable - explanation is the "dilution effect": the polymer phase volume increases over time. Allmost all the co-catalyst is absorbed within the polymer phase. Therefore, its concentration decreases with increasing yield. This leads to a decreasing catalyst activity, which we see as "decay".

Probably all these factors work together, for example: By extrapolating Figure 5.8 to zero decay, one can expect that if the ethylene pressure reaches 1.5 bars, the decay will vanish, which can be explained in terms of a sufficiently high co-catalyst back-diffusion at a low convective polymer flow (i.e. low pressure, i.e. low Rp). However, one should take into account that the fragmentation is limited at 1.5 bar ethylene pressure as discussed above - a lower number of active sites stays longer active having the same amount of co-catalyst available. 


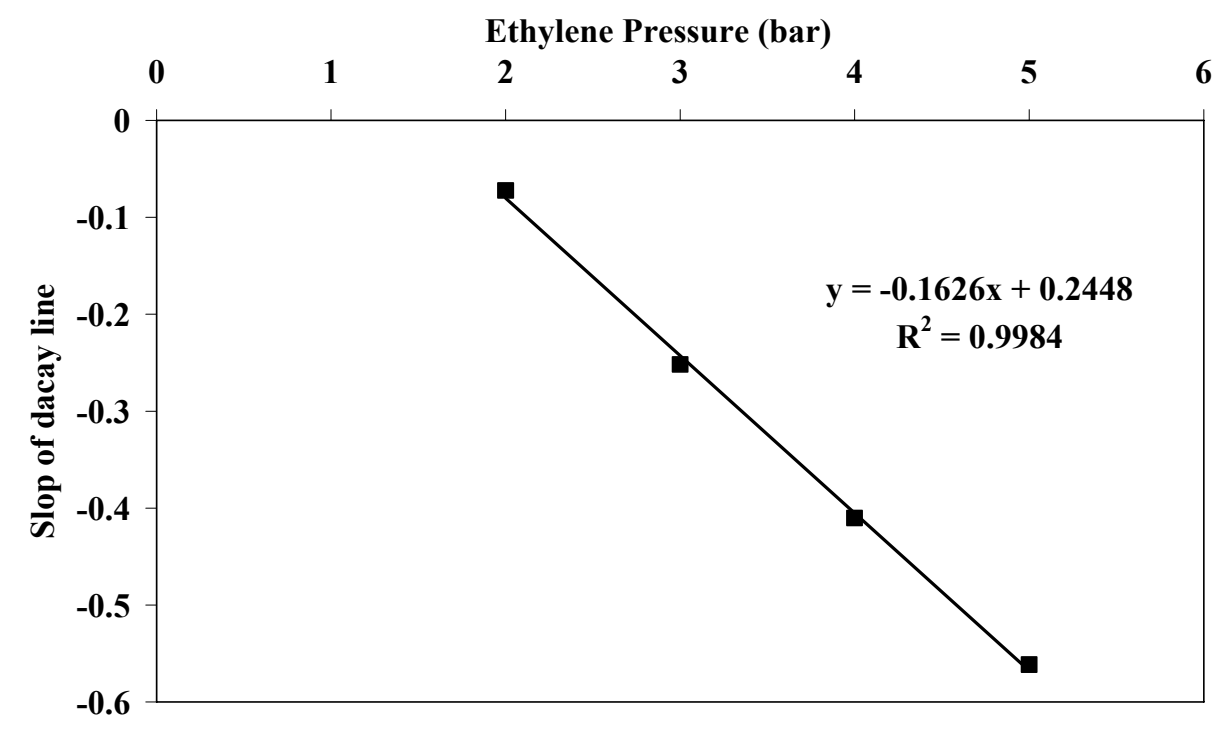

Figure 5.8-Plot of the slop of decay line versus ethylene pressure based on the results obtained by

Figure 5.7

The slope of the initiation rate curves, extracted from Figure 5.9 and shown in Figure 5.10, shows an exponential ethylene pressure dependency. This exponential behaviour can be explained by faster fragmentation accompanied by the faster generation of new active sites - a form of auto-acceleration until all potential active sites are activated.

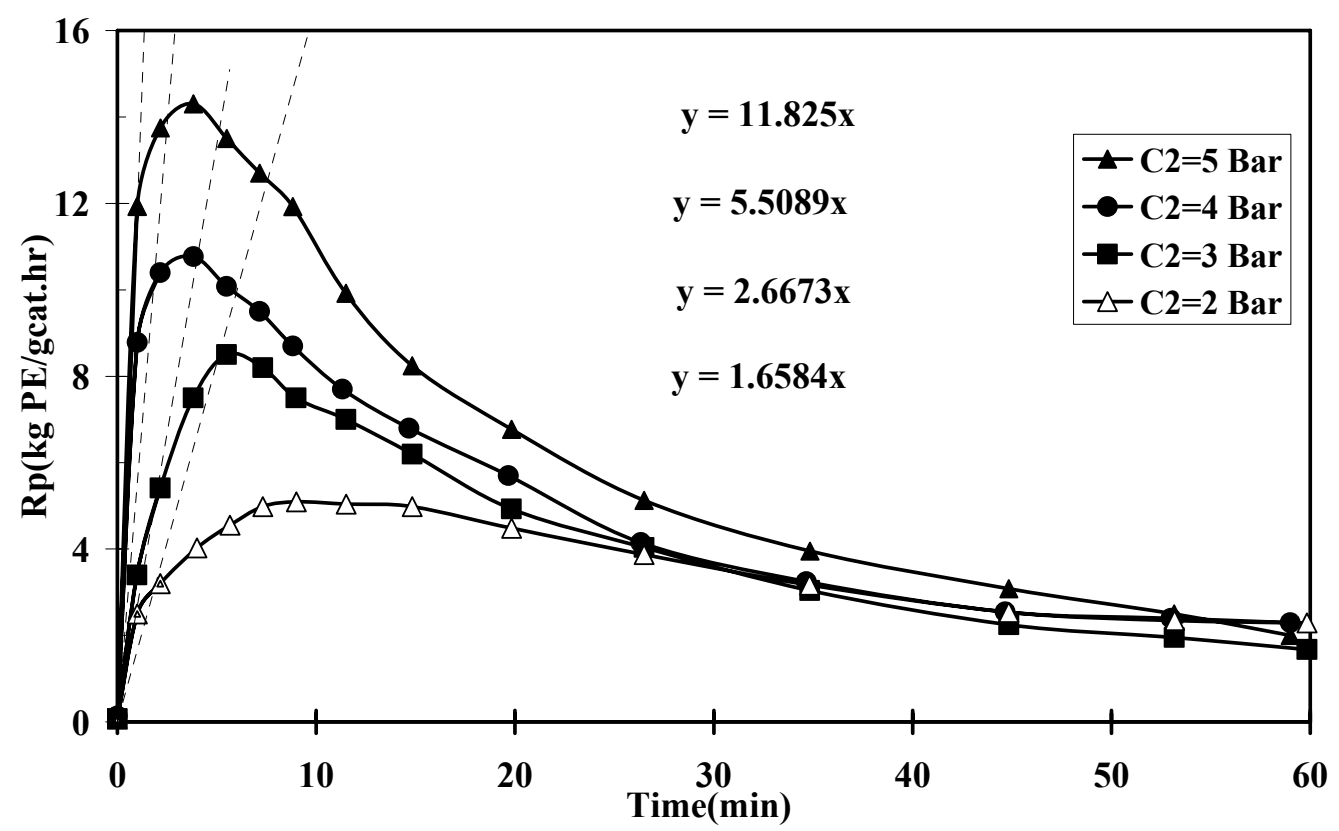

Figure 5.9-Effect of ethylene pressure on the reaction rate-profile of gas-phase ethylene polymerization using Ziegler-Natta catalyst at $\mathrm{T}=\mathbf{7 5}^{\circ} \mathrm{C}$, and the plot of the relevant decay line 


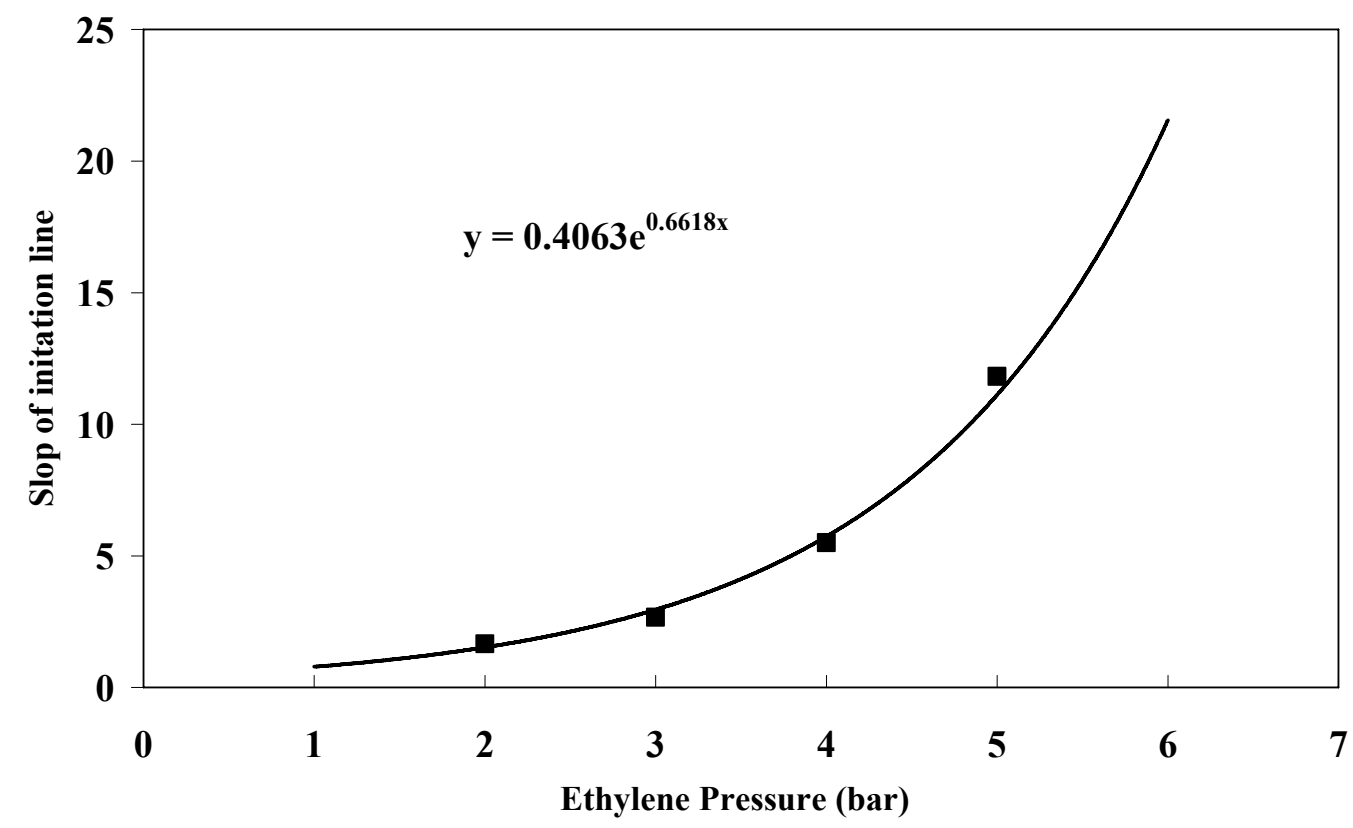

Figure 5.10-Plot of the slope of initiation line versus ethylene pressure based on the results obtained from Figure 5.9

Table 5.5 compares the results of the four experiments including the peak polymerization rate $\left(\operatorname{Max} R_{P}\right), \mathrm{Mw}, \mathrm{Mn}$, polydispersity, first and second level of crystallinity and melting temperature of powders produced in each run.

Table 5.5-Comparison of the properties of polymer obtained in ethylene series

\begin{tabular}{cccccccc}
\hline Run & Max $\mathrm{R}_{\mathrm{P}}{ }^{*}$ & $\begin{array}{c}\mathrm{M}_{\mathrm{w}} \\
(\mathrm{kg} / \mathrm{mol})\end{array}$ & $\begin{array}{c}\mathrm{M}_{\mathrm{n}} \\
(\mathrm{kg} / \mathrm{mol})\end{array}$ & $\mathrm{M}_{\mathrm{w}} / \mathrm{M}_{\mathrm{n}}$ & $\begin{array}{c}\mathrm{X}_{\mathrm{C} 1} \\
\%\end{array}$ & $\begin{array}{c}\mathrm{X}_{\mathrm{C} 2} \\
\%\end{array}$ & $\begin{array}{c}\mathrm{Tm} \\
\left({ }^{\circ} \mathrm{C}\right)\end{array}$ \\
\hline 1- $\mathrm{P}_{\mathrm{C} 2}=2$ bar & 5.16 & 191.4 & 24.8 & 7.7 & 65.8 & 70 & 134.4 \\
2- $\mathrm{P}_{\mathrm{C} 2}=3$ bar & 8.5 & 182.9 & 23.2 & 7.9 & 66.9 & 70.9 & 134.1 \\
3- $\mathrm{P}_{\mathrm{C} 2}=4$ bar & 11 & 193.3 & 24.5 & 7.9 & 61.1 & 66.3 & 135.8 \\
4- $\mathrm{P}_{\mathrm{C} 2}=5$ bar & 15.1 & 256.7 & 26.1 & 9.8 & 65 & 67.7 & 136.7 \\
\hline
\end{tabular}

$* \mathrm{R}_{\mathrm{p}}$ is expressed as $\mathrm{kg} \mathrm{PE} /$ gcat.hr

The maximum Rp versus the ethylene pressure results in a first order dependence; see Figure 5.11. As in slurry experiments (see Figure 5.3) this $1^{\text {st }}$-order curve is also valid at low pressures since it begins at zero. The only difference is the slope and the "initiation time" of the polymerization rate curve, which we define as the time required to reach the maximum rate. The slope is higher and the initiation time is shorter than in the case for gas-phase polymerization. This is in perfect agreement with our hypothesis as explained in Chapter 3(section 3.3). 


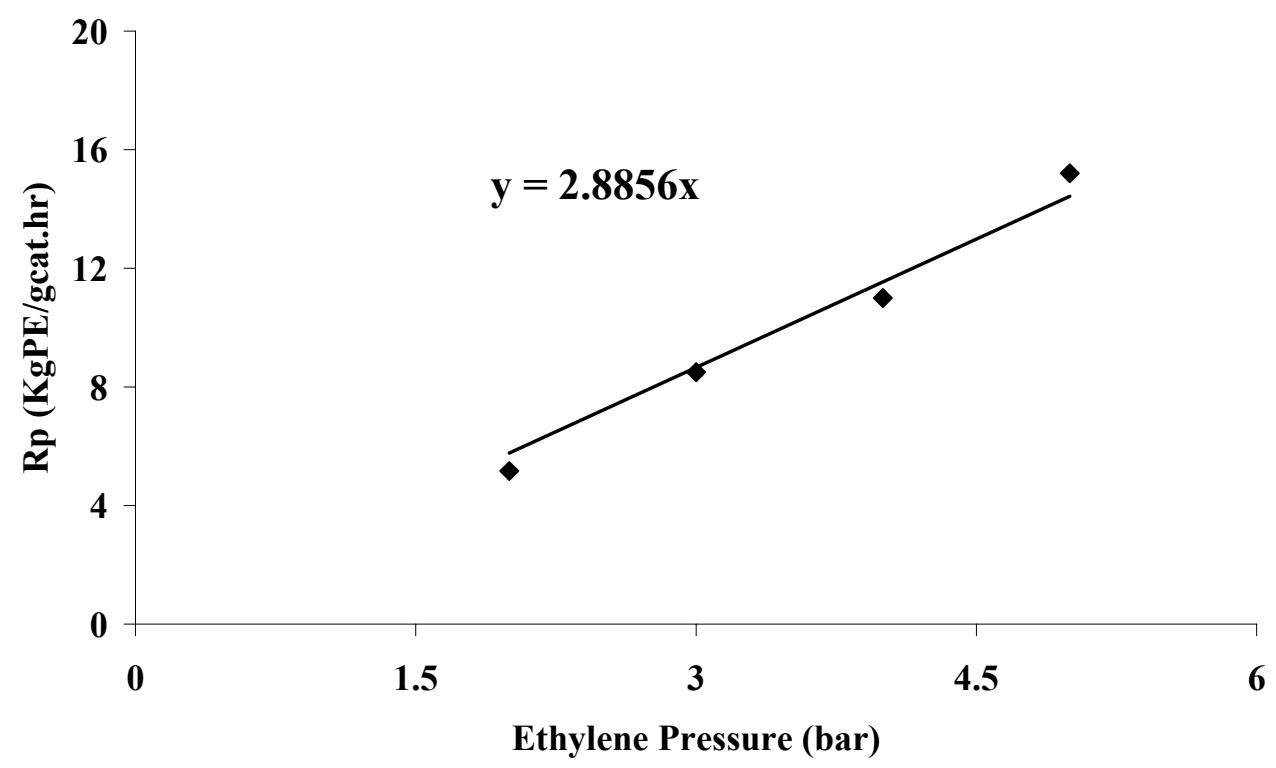

Figure 5.11- Polymerization rate as function of ethylene pressure

In terms of molecular weight, Table 5.5 and Figure 5.12 do not show significant changes when the ethylene pressure increases from 2 bar to 5 bar. This is an unexpected result, because the hydrogen : monomer ratio decreases from 1 to 0.2 (so by a factor of 5) and most of the chains are terminated by hydrogen transfer. We will extensively discuss the reasons in section 5.4.2.

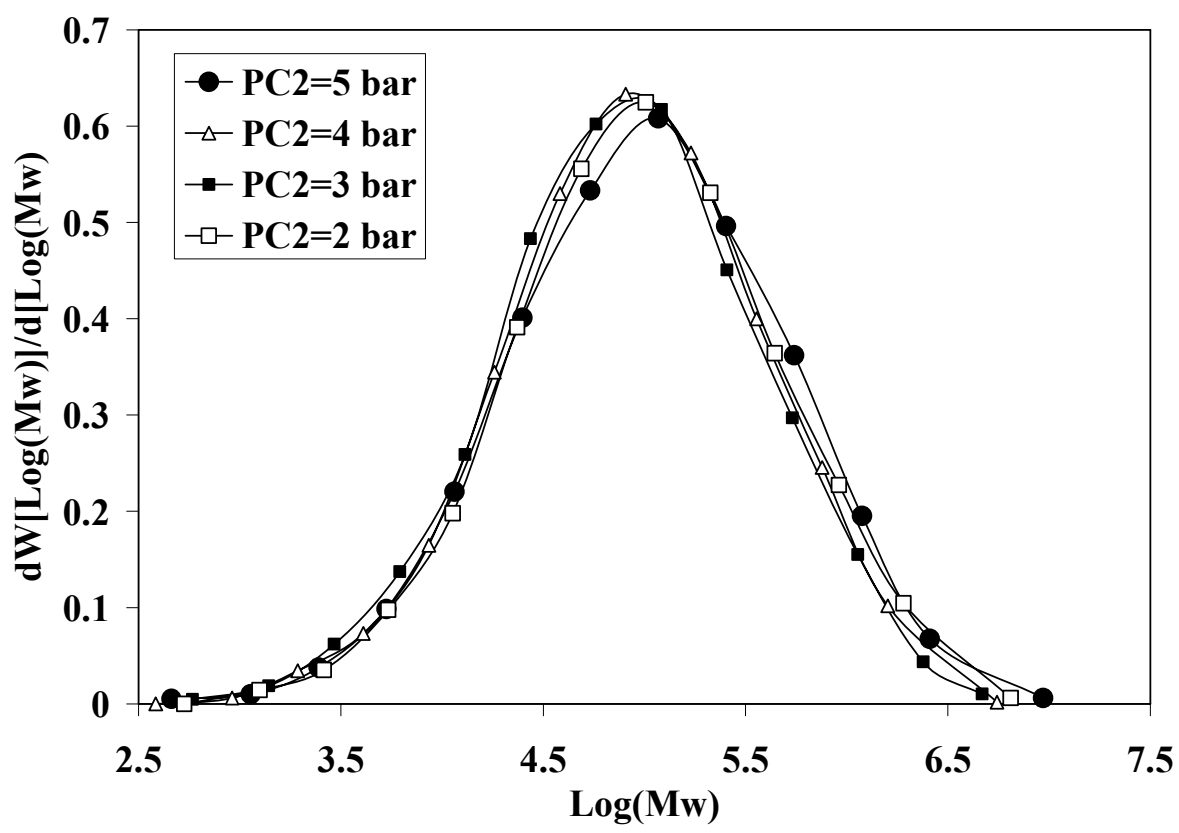

Figure 5.12- MWDs of the polymer produced in gas-phase ethylene polymerization at constant hydrogen pressure $\left(\mathbf{P}_{\mathrm{H} 2}=2 \mathrm{bar}\right)$; see also Table 5.5

Table 5.5 also reveals that increasing the ethylene pressure at a constant hydrogen pressure has no significant influence on crystallinity. The crystallinity does not reach the critical figures ( $70 \%$ for $\mathrm{X}_{\mathrm{C} 1}$ and $73 \%$ for $\mathrm{X}_{\mathrm{C} 2}$ ). This could be the reason why the particle size 
distributions remain unchanged (see Figure 5.13) despite the large changes in growth stress (see Figure 5.7).

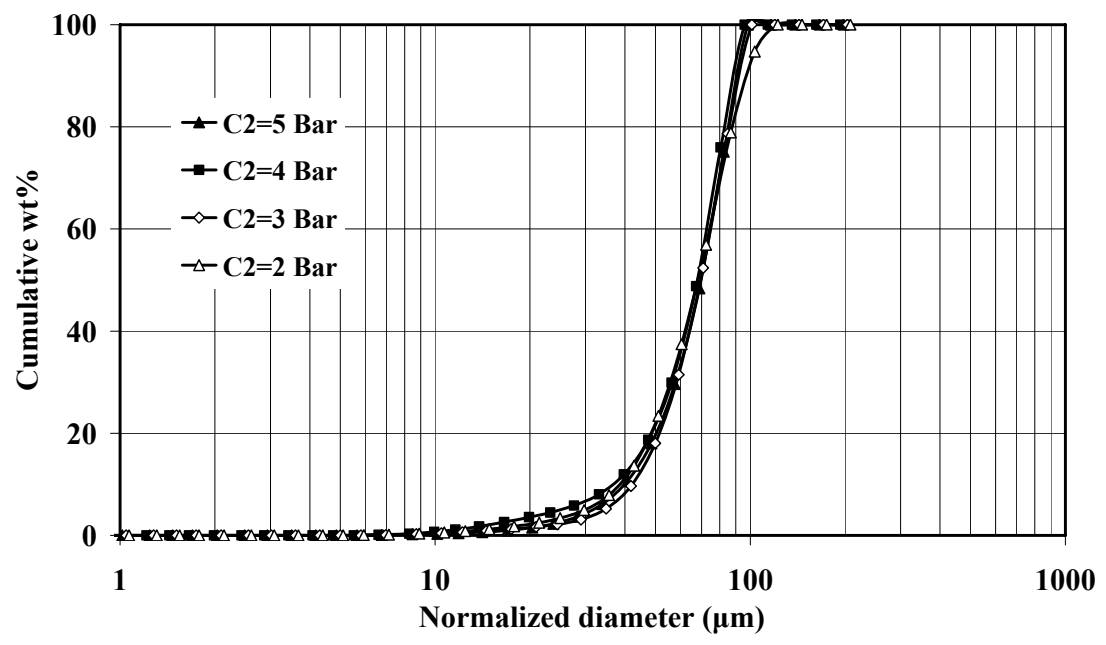

Figure 5.13- Effect of ethylene pressure on PSD, see also Table 5.5

\subsection{Slurry polymerization at constant $\mathbf{P}_{\mathrm{H} 2} / \mathbf{P}_{\mathrm{C} 2}$ ratio}

Increasing the hydrogen content in a slurry or gas-phase reactor decreases the molecular weight, leading in turn to a broad MWD. This molecular weight regulation and rate behaviour leads to fines production, which is more pronounced for slurry polymerization.

Furthermore, the results obtained from the ethylene series experiments at a constant hydrogen pressure reveals that higher ethylene pressure does not influence the particle size distribution although it does increase substantially the productivity of the catalyst. Additionally, by increasing the ethylene pressure, the MWD becomes narrower and the MWD shifts slightly to higher molecular weights. One can conclude that, by increasing the ethylene pressure at constant $\mathrm{P}_{\mathrm{H} 2} / \mathrm{P}_{\mathrm{C} 2}$, one can reach a higher productivity with better tailoring of the molecular weight and with less influence on particle size distribution.

The experimental details of the polymerization were the same as for standard slurry experiments performed at $\mathrm{T}=80^{\circ} \mathrm{C}$ which have already been described in Chapter 2. The polymer properties are also measured with the same methods and devices, as explained in Chapter 2. Some more experimental conditions and initial results are given in Table 5.6.

Table 5.6- Operating conditions and polymer properties of four experiments in slurry

\begin{tabular}{cccccccccc}
\hline Run & $\begin{array}{c}\mathrm{P}_{\mathrm{C} 2} \\
(\text { bar })\end{array}$ & $\begin{array}{c}\mathrm{P}_{\mathrm{H} 2} \\
(\text { bar })\end{array}$ & $\begin{array}{c}\mathrm{R}_{\mathrm{P}}{ }^{*} \\
\text { average }\end{array}$ & $\begin{array}{c}\mathrm{Tm} \\
\left({ }^{\circ} \mathrm{C}\right)\end{array}$ & $\begin{array}{c}\mathrm{X}_{\mathrm{C} 1} \\
\%\end{array}$ & $\begin{array}{c}\mathrm{X}_{\mathrm{C} 2} \\
\%\end{array}$ & $\begin{array}{c}\mathrm{M}_{\mathrm{w}} \\
(\mathrm{kg} / \mathrm{mol})\end{array}$ & $\begin{array}{c}\mathrm{M}_{\mathrm{n}} \\
(\mathrm{kg} / \mathrm{mol})\end{array}$ & $\mathrm{M}_{\mathrm{w}} / \mathrm{M}_{\mathrm{n}}$ \\
\hline GSE1 & 4 & 4 & 6.91 & 134.1 & 67.9 & 74.4 & 117.1 & 11.8 & 9.9 \\
GSE2 & 2 & 2 & 4.25 & 134.4 & 68.5 & 73.9 & 156.7 & 12.3 & 12.7 \\
GSE3 & 1 & 1 & 2.28 & 133.3 & 68.3 & 72.6 & 215.3 & 15.9 & 13.6 \\
GSE4 & 2 & 4 & 4.48 & 132.5 & 72 & 78 & 83.5 & 7.1 & 11.7 \\
\hline
\end{tabular}

* $\mathrm{R}_{\mathrm{p}}$ in $\mathrm{kg} \mathrm{PE} /$ gcat.hr 
As can be seen from Figure 5.14, all rate profiles show a rate build up type. By increasing the ethylene and hydrogen pressure from 1 to 4 bar, the initiation period for reaching the peak decreases.

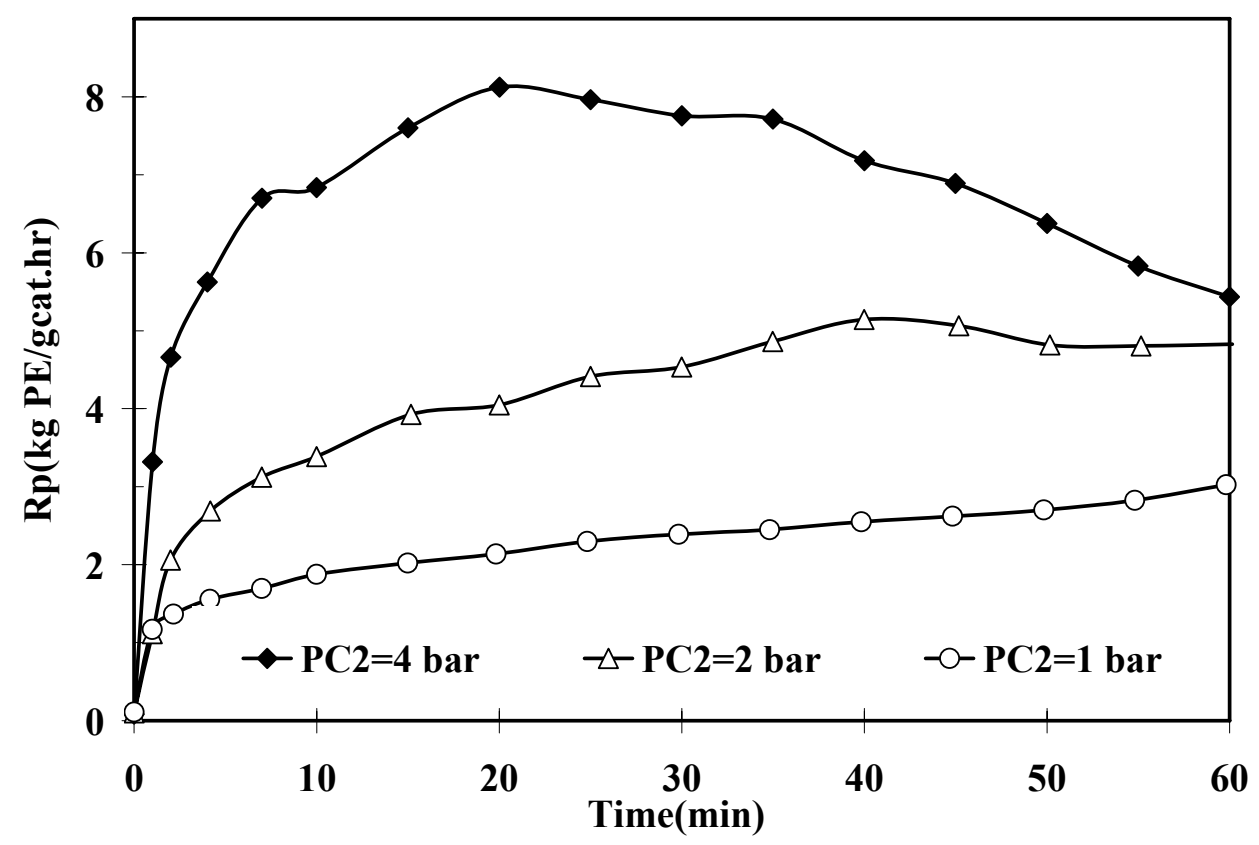

Figure 5.14-Reaction rate profiles for ethylene slurry polymerization at $80^{\circ} \mathrm{C} ; \mathbf{P}_{\mathrm{H} 2} / \mathbf{P}_{\mathrm{C} 2}=1$

Faster activation followed by faster deactivation is more pronounced for higher ethylene pressure. Fast activation can be attributed to higher ethylene participation in activation, as discussed for the two previous series (faster fragmentation accompanied by faster active site generation). However, the decay behaviour is neither similar to that for the first series (no decay) nor for the second series (quick decay), but lies somewhere in-between. Due to the participation of hexane, the concern about overheating can be neglected. However, the mass transfer limitation of co-catalyst and the dilution effect as discussed for the gasphase series can not be ignored. The structure of the polymer changes from lower to higher crystalline in the presence of hydrogen, which hinder co-catalyst back-diffusion.

As can be deduced from Table 5.6, increasing the ethylene pressure from 1 to 4 bar for the first three experiments leads to the following changes:

- significant increase in the average yield of catalyst from 2.28 to $6.91 g_{P E} /\left(g_{c a t} \cdot h\right)$

- slight increase in $2^{\text {nd }}$ crystallinity (but un-remarkable for $1^{\text {st }}$ crystallinity) and also in the melting temperature(Tm)

- a large decrease in Mw and Mn accompanied by less broadening from 13.6 to 9.9.

Surprisingly, comparing the results from runs GSE1 with GSE4 reveal that increasing the ethylene pressure at constant hydrogen pressure:

- Increases Mw and Mn

- Decreases the narrowness of MWD

- Decreases the crystallinity 
Based on equation 5.8 "which incorporates the hydrogen effect", one would expect an increasing molecular weight as the ethylene pressure increases at a constant hydrogen:ethylene ratio, but this does not happen.

How can this discrepancy be explained?

First, we remember that the concentrations in Equation. 5.8 are the concentrations near the active site, which can be completely covered by the polymer phase:

$$
\frac{1}{M_{n}} \approx \frac{k_{t M}}{k_{p}}+\frac{k_{t A}[A]}{k_{p}[M]}+\frac{k_{t S}}{k_{p}[M]}+\frac{k_{t H_{2}}\left[H_{2}\right]}{[M]} \ldots \ldots
$$

Second, it is well known that a polymer network expands by swelling as the ethylene pressure increases. It is also well known that in multi-component systems the thermodynamic properties - as well as transport properties of one component - are usually influenced by the other components.

From our results, we conclude that the presence of monomer in the polymer phase increases the solubility (and perhaps the diffusion rate) of hydrogen. This effect is taken into account by a solubility function as follows:

$$
\begin{array}{ll}
{\left[H_{2}\right] \approx P_{H_{2}} f_{H_{2}}} & \text { Equation } 5.9
\end{array}
$$

with the solubility function proportional to the monomer pressure:

$$
f_{H_{2}} \approx P_{C_{2}}^{m}
$$

Of course, we assume a positive order $m$.

By substituting equation 5.9 and 5.10 into equation 5.8 with exchanging concentrations by pressures, we can write:

$$
\frac{1}{M_{n}} \approx \frac{k_{t M}}{k_{p}}+\frac{k_{t A}[A]}{k_{p} P_{C_{2}}}+\frac{k_{t S}}{k_{p} P_{C_{2}}}+k_{H_{2}} P_{H_{2}} P_{C_{2}}^{(m-1)} \ldots \ldots . . \quad \text { Equation } 5.11
$$

For those experiments with a constant hydrogen: ethylene pressure ratio, X, we obtain:

$$
\frac{1}{M_{n}} \approx \frac{k_{t M}}{k_{p}}+\frac{k_{t A}[A]}{k_{p} P_{C_{2}}}+\frac{k_{t S}}{k_{p} P_{C_{2}}}+k_{H_{2}} X . P_{C_{2}}^{m} \cdots \cdots
$$

In this way, the influence of increasing monomer pressure is seen to be more complex than is usually assumed. Table 5.7 shows various cases which lead to an increased, decreased or constant $\mathrm{M}_{\mathrm{w}}$ as the ethylene pressure increases. 
Table 5.7-Effect of ethylene pressure on $M_{W}$ at different values of $P_{H 2}$ and $X^{*}$

\begin{tabular}{|c|c|c|}
\hline $\begin{array}{c}\text { Experimental } \\
\text { Mode }\end{array}$ & CASE & Molecular weight response for increasing $\mathbf{P}_{\mathrm{C} 2}$ \\
\hline \multirow{3}{*}{$\begin{array}{l}\mathbf{P}_{\mathbf{H} 2}=\text { constant } \\
\text { see equation. } 5.11\end{array}$} & $\mathrm{~A}: \mathrm{P}_{\mathrm{H} 2}=0$ & “increasing” \\
\hline & $\begin{array}{l}\mathrm{B}: \quad \mathrm{P}_{\mathrm{H} 2}>0 \\
0<\mathrm{m}<1\end{array}$ & "increasing" \\
\hline & $\begin{array}{l}\mathrm{C}: \mathrm{P}_{\mathrm{H} 2}>0 \\
\mathrm{~m}>1\end{array}$ & $\begin{array}{l}\text { At low } \mathrm{P}_{\mathrm{H} 2} \text { : "increasing", but...with increasing } \mathrm{P}_{\mathrm{H} 2} \text { : } \\
\text { term } 4 \text { in equation } 5.11 \text { becomes increasingly } \\
\text { dominant - this can overrule the increasing effect of } \\
\text { terms } 2 \text { and } 3 \text {. The final response can be: "increasing", } \\
\text { "constant" or "decreasing" }\end{array}$ \\
\hline \multirow{2}{*}{$\begin{array}{l}\mathbf{X}=\text { const } \\
\text { see equation. } 5.12\end{array}$} & $\mathrm{D}: \quad \mathrm{X}=0$ & "increasing" \\
\hline & $\mathrm{E}: \quad \mathrm{X}>0$ & $\begin{array}{l}\text { At low } \mathrm{X} \text { and low } \mathrm{P}_{\mathrm{C} 2} \text { : "increasing", but...with } \\
\text { increasing } \mathrm{X} \text { : term } 4 \text { in equation } 5.12 \text { becomes } \\
\text { increasingly dominant }- \text { this can overrule the } \\
\text { increasing effect of terms } 2 \text { and } 3 \text {. The final response } \\
\text { can be: "increasing" "constant" or "decreasing" }\end{array}$ \\
\hline
\end{tabular}

* $\mathrm{X}$ is hydrogen: ethylene pressure ratio

One should always keep in mind that the above equations are derived for a single-site catalyst. The different sites of the used Z-N catalyst can show various responses, but principally each site is expected to follow these equations (although probably with different rate constants, of course).

Decreasing the molecular weight as a result of increasing the ethylene pressure for the first three runs can be explained by case E in Table 5.7 It seems that increasing the ethylene pressure at a constant hydrogen : ethylene ratio, X, term 4 overrules the others terms in equation 5.12, in turn leading to a decrease of the molecular weight.

Table 5.6 shows that the crystallinity slowly increases due to decreasing the molecular weight as a consequence of increasing the ethylene pressure. The difference in molecular weight does not change the crystallinity. On the other hand, comparison of the results of GSE4 with those from other experiments reveals that the molecular weight for the GSE4 run is very low (less than $100 \mathrm{~kg} / \mathrm{mol}$ for $M_{w}$ or $10 \mathrm{~kg} / \mathrm{mol}$ for $M_{n}$ ) Consequently. the higher crystallinity and lower melting temperature could be reasonably expected for the GSE4 run due to the significantly low molecular weight. Figure 5.15 shows a comparison of the molecular weight distribution measured from these four experiments. It is quite clear for GSE1, GSE2 and GSE3 that the MWD of polymer produced at higher ethylene pressure is shifting towards lower molecular weight - the molecular weight increasing effect of higher ethylene pressures is overcompensated by the solubility function of $\mathrm{H}_{2}$. Comparing the MWD 
of GSE1 $\left(4 \mathrm{barC}_{2}+4\right.$ bar $\left.\mathrm{H}_{2}\right)$ with GSE4 $\left(2\right.$ bar $\mathrm{C}_{2}+4$ bar $\left.\mathrm{H}_{2}\right)$ indicates that the MWD follows the standard expectations: the $100 \%$ higher $\mathrm{H}_{2}: \mathrm{C}_{2}$ ratio produces lower $\mathrm{Mw}$.

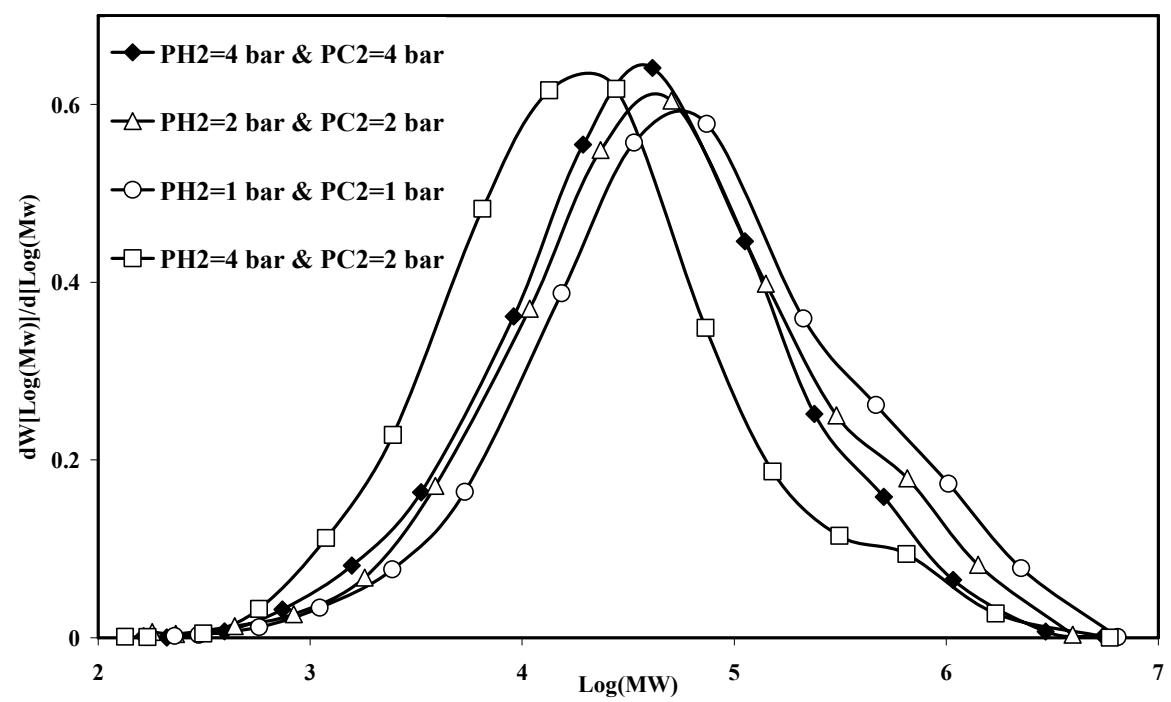

Figure 5.15-MWDs of the polymer produced in four experiments given in Table 5.6

One may expect that for the experiments with $\mathrm{P}_{\mathrm{H} 2} / \mathrm{P}_{\mathrm{C} 2}=1$, increasing ethylene pressure increases dramatically the internal stress [66] due to increasing growth stress. This increasing in internal stress should lead to faster fragmentation and - above a critical limit - to more particle disintegration, especially for the extreme case GSE1. According to Figure 5.16, this is the case.

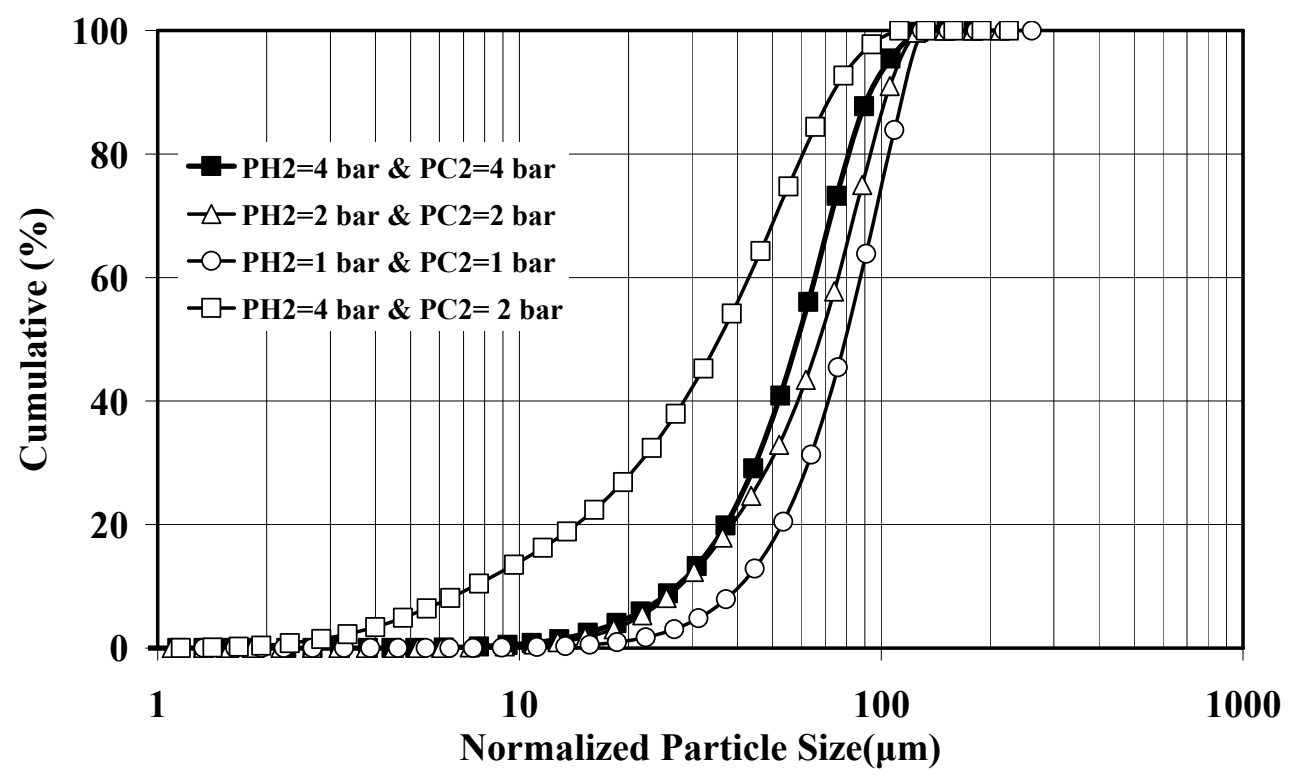

Figure 5.16-Comparison of cumulative PSD profiles normalized with the yield of the polymer extracted from four experiments given in Table 5.6 
Also run GSE4 with $100 \%$ higher $\mathrm{H}_{2}: \mathrm{C}_{2}$ ratio, meets the expectations: the highest degree in particle disintegration is observed for the highest crystallinity $(73 \%)$ - the lower growth stress does not compensate this effect

\subsection{Conclusions}

\section{For slurry polymerization in the absence of hydrogen:}

- The "build-up" profiles were obtained without any decay. A sufficiently good backdiffusion of co-catalyst could be the reason.

- The ratio of the initial slope of the rate curves obtained in two experiments with different ethylene pressure shows higher values compared to the ethylene pressure ratio. We attributed this to faster fragmentation accompanied by faster generation of new active sites at higher ethylene pressure during the initial phase of polymerization.

- The initial fragmentation seems to be the reason for a higher-than-first order regarding monomer pressure at low pressures.

- Experiments with stepwise increase of ethylene pressure confirmed the first order ethylene pressure dependency.

- As ethylene pressure increases, the weight and number average molecular weight increases slightly. This was explained in two ways: the Mw-increasing influence of ethylene pressure in equation 5.7 and deconvolution, which shows the low molecular weight producing centres, contribute less compared to those that produce high molecular weight.

- A small shift of the PSD profile towards small particles was seen as ethylene pressure increases. This can be attributed to higher growth stress. The shift is more pronounced for bigger particles compared to smaller particles. Big particles can easier disintegrate due to higher stress accumulation.

\section{For gas-phase polymerization in the presence of hydrogen:}

- The "decay" profiles were obtained. The higher the monomer pressure, the higher the peak activity and the faster the decay.

- The slope of the decay rate curves shows linear dependency on ethylene pressure. Three reasons were given: the overheating during the initial phase, the mass transfer behaviour of the co-catalyst and the dilution effect.

- The slope of the initiation rate curves shows an exponential ethylene pressure dependency. We explained this by a form of auto-acceleration mechanism involving "faster fragmentation accompanied by faster generation of new active sites" due to higher ethylene pressure and higher temperature at the particle level.

- The first order reaction with respect to ethylene pressure is also valid.

- Compared to slurry, the gas-phase experiments show the higher initial slope of the initiation accompanied by a faster initiation time. This can be explained by 
the overheating of the locally growing particle and co-catalyst participation in the activation processes.

- Despite the fact that the hydrogen: ethylene pressure ratio decreases as the ethylene pressure increases, no significant changes are observed in terms of MWD, $M_{w}$ and $M_{n}$. It was shown that increasing ethylene pressure might increase the solubility of hydrogen in the polymer structure leading to termination of more chain by hydrogen transfer.

- Despite the substantial change in growth stress as the ethylene pressure increases, the crystallinity does not reach to the critical figure, resulting no changing in the PSD profiles.

\section{For ethylene series experiments at constant hydrogen: ethylene ratio:}

- Higher ethylene pressure, leads to faster activation followed by faster deactivation. The cause of faster activation is already explained, see above. The faster deactivation was attributed mainly to the production of polymer around active sites, which can

o limit the back-diffusion of the co-catalyst and

o cause a dilution effect.

- significant increase in the average yield of the catalyst

- nearly constant crystallinity and melting temperature (Tm) as a consequence of the dominant hydrogen transfer

- large decrease in $\mathrm{Mw}$ and $\mathrm{Mn}$ accompanied by significant narrowing of the MWD; this behaviour can be explained by introducing a "solubility function" that explains why the hydrogen concentration increases with increasing ethylene pressure as shown in equation 5.12; the change in molecular weight of all three series described in this chapter can be explained by equation 5.12 .

- fines generation (particle disintegration) was negligible within the parameters varied. 


\section{Chapter 6}

\section{Influence of Hydrogen}

\subsection{Introduction}

The molecular weight and MWD of polyethylene produced using Z-N catalysts can be controlled and regulated by many variables such as catalyst, co-catalyst, monomer, hydrogen, and temperature (5-6). Hydrogen is the strongest and fastest chain transfer agent for controlling the MWD and can significantly affect the reaction rate of ethylene polymerization [83, 84]. Some other hydrogen effects on polymer properties, such as crystallinity, melting temperature and elongation at break have also been reported in the literature [85].

To facilitate better comparison and explanation of the new results achieved in this work, some important published results were selected as described below.

In 1959, Natta et al [66] were the first to describe the chemical effect of the influence of hydrogen on reaction rate profiles and molecular weight by proposing a chain transfer mechanism, as expressed in reaction 6.1, for depressing the reaction rate by introducing hydrogen. Assuming a slow re-initiation mechanism, as expressed in reaction 6.2, the reversible rate-decreasing effect of hydrogen becomes understandable - removing the hydrogen can lead to rate enhancement again. The authors assumed that the reaction of ethylene insertion into the metal-carbon bond (reaction 6.3) is much faster than the ethylene insertion into the metal-hydrogen bond (reaction 6.2).

$$
\begin{array}{ll}
\text { Cat }-P_{n}+H_{2} \stackrel{k_{H}}{\longrightarrow} \text { Cat }-H+D_{n} & \text { Reaction } 6.1 \\
\text { Cat }-H+E \stackrel{k_{1}}{\longrightarrow} \text { Cat }-P_{1} & \text { Reaction } 6.2 \text { (slow) } \\
\text { Cat }-P_{n}+E \stackrel{k_{2}}{\longrightarrow} \text { Cat }-P_{n+1} & \text { Reaction } 6.3 \text { (fast) }
\end{array}
$$

where; $k_{H}$ is the rate constant of chain transfer reaction with hydrogen; $k_{1}$ and $k_{2}$ are the rate constants for the reaction of ethylene insertion into catalyst-hydrogen bond and catalyst-carbon bond, respectively; $P_{n}$ is the living polymer; $D_{n}$ is the dead polymer; and $E$ represents ethylene.

Contradicting Natta's assumption of ethylene insertion, Yamamoto [86] and Brookhart and Lincoln [87] reported some experimental data which show that the ethylene insertion into the metal-hydrogen bond is faster than the reaction of ethylene insertion into the catalyst-carbon bond; therefore, the rate-decreasing effect of hydrogen cannot be explained by 
the different reaction rates of (6.2) and (6.3). On the other hand, a faster first incorporation step is not a satisfactory explanation for a polymerization rate-increasing effect.

In 1989, Kissin [18] investigated the effect of hydrogen pressure on the reaction rate profile of slurry and gas-phase ethylene polymerization using a typical $\mathrm{TiCl} 4 / \mathrm{MgCl}$-based Ziegler catalyst supported on silica and found the same reversible rate-depressing effect as Natta had found. He proposed the "reversible hydrogen deactivation of polymerization centers" given in reaction 6.4 as the explanation:

$$
\mathrm{C}^{*}+\mathrm{H}_{2} \underset{\mathrm{k}_{-3}}{\rightleftharpoons} \mathrm{C}_{\mathrm{H}_{2}}^{*} \quad \text { Reaction } 6.4
$$

where $C^{*}$ is the number of active centers in a reaction, $C^{*}{ }_{\mathrm{H} 2}$ stands for temporarily deactivated centers by hydrogen in the system, and $k_{3}$ and $k_{-3}$ are rate constants.

Kissin also proposed in 1999 [76] another scheme (see Scheme 6.1) based on the assumption that $\mathrm{Ti}-\mathrm{C}_{2} \mathrm{H}_{5}$ bonds are generated by various mechanisms (e.g., chain transfer to ethylene, ethylene insertion into $\mathrm{Ti}-\mathrm{H}$ bond) which is stable. Kissin assumed that the stability of the $\mathrm{Ti}-\mathrm{C} 2 \mathrm{H} 5$ bond is the result of an unusually strong $\beta$-agostic interaction between the hydrogen atom of its methyl group and the Ti atom. The rate-depressing effect of hydrogen in ethylene polymerization reactions was explained by the immediate formation of the $\beta$-agostic coordinated $\mathrm{Ti}-\mathrm{C} 2 \mathrm{H} 5$ group.

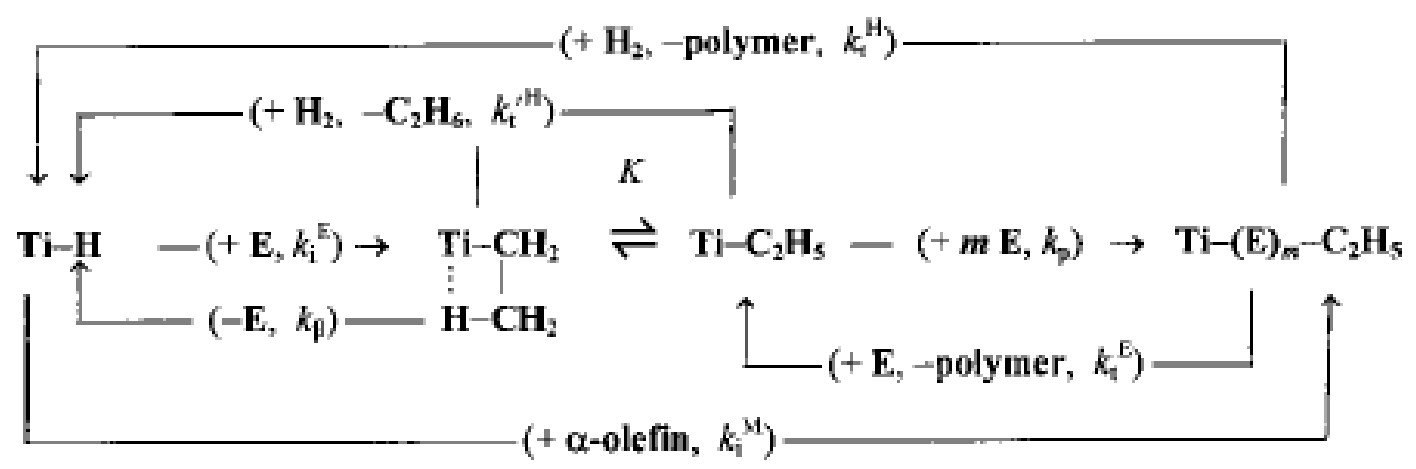

Scheme 6.1-The Kinetic Mechanism proposed by Kissin, taken from [76]

Some other published studies contradict these hypothesises. A slight rate enhancement in ethylene polymerization by hydrogen addition using a $\mathrm{MgCl} 2$ supported titanium catalyst was reported by Marques et al (1993) [35] at hydrogen pressures below 2 bar. They attributed this effect to better accessibility of the monomer to the active centre due to the decreasing molecular weight in the presence of hydrogen. Boucheron (1975) [88] used the same hypothesis to explain the rate increasing by hydrogen.

E. M. Pijpers and B. C. Roest (1972) [89] proposed that polymer chains cannot migrate from the active centres in the absence of hydrogen, because they contain a terminal double bond capable of competing with the monomer in the formation of a $\pi$-complex with the active site. Introducing hydrogen would decrease the amount of terminal double bonds, since the chains migrate easily and consequently the activity is increased.

Zakharov et al. (2001) [90] studied the effect of adding hydrogen and co-catalysts during slurry ethylene polymerization using supported vanadium-magnesium catalysts 
(VMC) with the compositions $\mathrm{VCl} / \mathrm{MgCl} 2$ and $\mathrm{VOCl} / \mathrm{MgCl} 2$. They reported the same results as Natta and Kissin, but suggested another explanation that took into account the role of co-catalyst and monomer. They considered that the chain transfer with hydrogen via reaction 6.6 is followed by production of alkyl aluminium hydride by surface reaction (reaction 6.7), and suggested the formation of temporarily inactive "dormant" sites via reaction 6.8. By removing hydrogen, reactivation of the catalyst by ethylene may happen by conversion of dialkylaluminumhydride to trialkyl aluminium via reaction 6.9.

Clearly, the higher the ethylene pressure, the faster the reactivation.

$$
\begin{array}{lr}
V-P+H_{2} \rightarrow V-H+\text { Polymer } & \text { Reaction } 6.6 \\
V-H+A^{A l R_{3}} \rightarrow V-R+\text { AlR }_{2} H & \text { Reaction } 6.7
\end{array}
$$

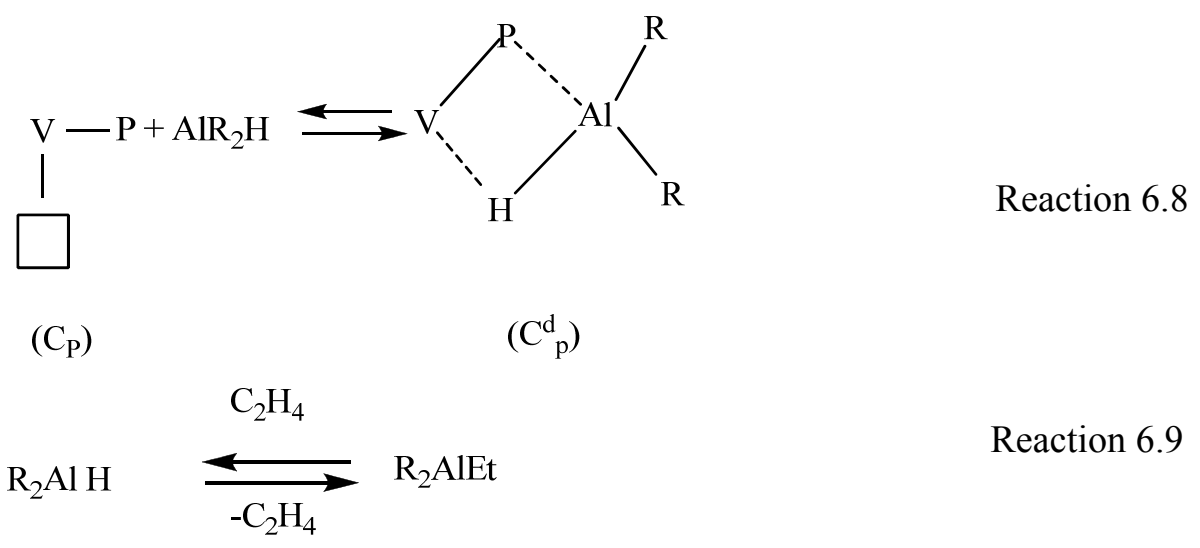

In 1997, Jerome et al. [85] reported on the influence of hydrogen on the rate of reaction, molecular weight, crystallinity, and physico-mechanical properties of the final polyolefin produced in slurry ethylene polymerization using various Ziegler-Natta catalysts. They found that by increasing the partial pressure of hydrogen in the bulk gas phase of the slurry reactor, the reaction rate, molecular weight and melting temperature of the HDPE polymer formed decreased and, in parallel, crystallinity and elongation at break also increased.

Although, there has been considerable research presented in the literature concerning the influence of hydrogen on the rate of reaction (decreasing, increasing or constant), MWD, crystallinity and melting temperature, most researchers focus mainly on chemical effects, while neglecting physical effects. Therefore, the question: "under which conditions one can expect a positive or a negative $\mathrm{H}_{2}$ response?" remains open. Beside the chemical effect found by Natta et al. - with different explanations by Kissin and Zhakarov ${ }^{1}$ - some indication is given about the role of physical factors: we believe that physical factors ${ }^{2}$ play an important

\footnotetext{
1 Another explanation is possible for rate acceleration by hydrogen: chain length dependent propagation constant (kp decreasing with chain length!) - This hypothesis is not yet discussed comprehensively in olefin polymerizations.

2 "physical factors" stands here for "thermodynamic equilibrium sorption/desorption and transport properties"
} 
role. However, these factors are very different in slurry versus gas-phase polymerization processes $[29,91]$.

Following the basic GRAF hypothesis, the investigation described in this chapter was carried out to compare, identify and precisely evaluate the influence of hydrogen on slurry and gas-phase ethylene homo-polymerization on the kinetics, MWD, crystallinity of produced polymer, and their interactions with particle fragmentation and disintegration.

\subsection{Experimental}

Generally, all methods and equipment are extensively described in Chapter 2.

Series of slurry and gas-phase homo-ethylene polymerization experiments were performed by increasing the hydrogen partial pressure in the bulk gas-phase of the reactor from 0 to 10 bar while keeping all other variables constant.

The common conditions at the start of the polymerizations were as follows:

- ethylene partial pressure: 2 bar

- polymerization temperature: $80^{\circ} \mathrm{C}$.

For the slurry experiments, $700 \mathrm{ml} \mathrm{n}$-hexane used as a solvent, mixed with $200 \mathrm{mg}$ TIBA as a scavenger for 15 minutes at reaction temperature.

For gas-phase experiments, $110 \mathrm{mg}$ salt $(\mathrm{NaCl}$ powder) used as a bed, mixed with $200 \mathrm{mg}$ TIBA as a scavenger for 15 minutes at reaction temperature. The reactions were performed under isothermal and isobaric conditions while keeping the reactor pressure constant by introducing ethylene via the mass flow meter.

\subsection{Results and Discussion}

\subsubsection{Polymerization Rate Profiles}

Figure 6.1 and Figure 6.2 respectively show the polymerization rate-time curves obtained for ethylene homo-polymerization with or without hydrogen in gas-phase and slurry. The rate-time profiles in gas-phase show a decay type rate-time behavior in which the rate rises rapidly, reaching a maximum followed by a decreasing reaction rate over time.

Conversely, the profiles in slurry polymerization show a build-up type profile behavior; the rate rises slowly, reaching a maximum and then decreasing very slowly or even remaining constant. The faster and higher rate at the beginning of the reaction in gas-phase can be attributed to two reasons: first, higher local reaction temperatures at the particle level and second, higher local co-catalyst concentration around the active centers in the gas-phase. 


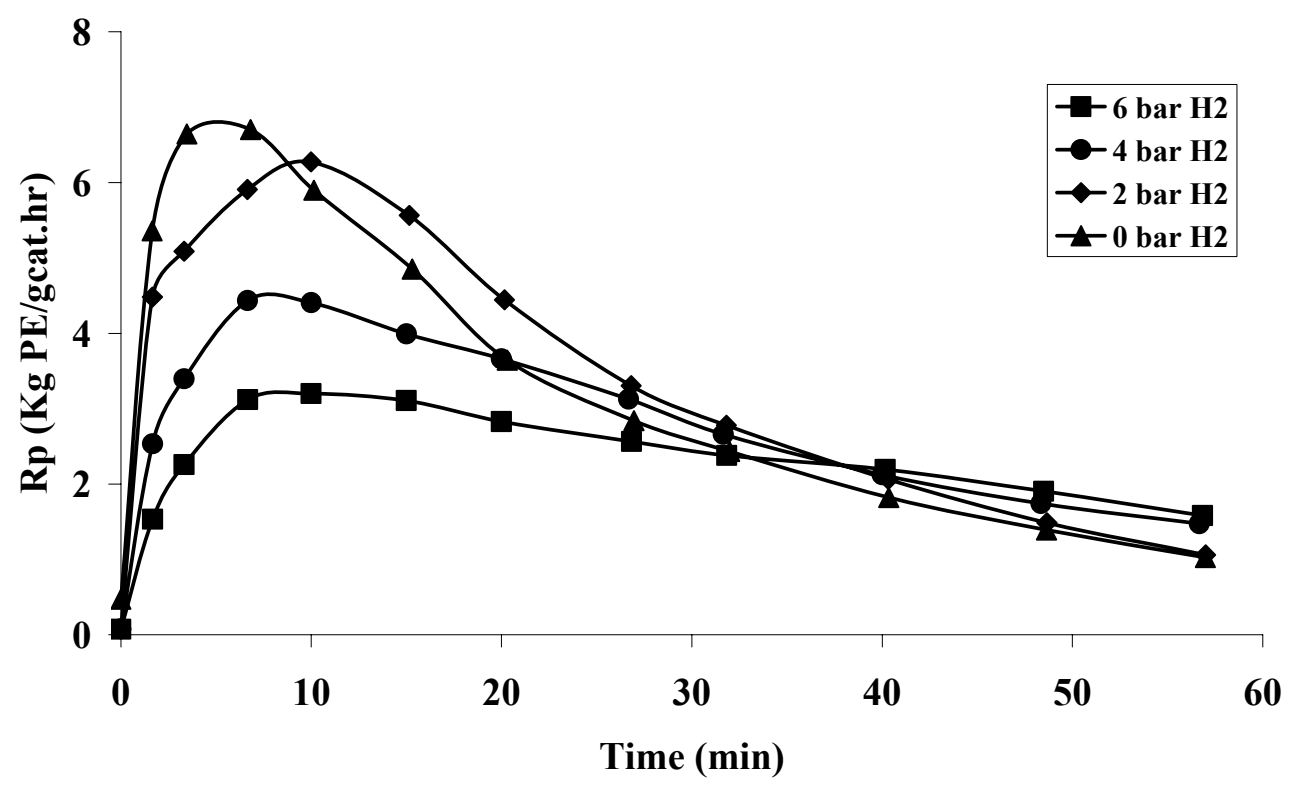

Figure 6.1-Hydrogen influence on reaction rate in gas-phase polymerization at $\mathrm{T}=80^{\circ} \mathrm{C}$ and

$$
\mathbf{P}_{\mathrm{C} 2}=2 \text { bar }
$$

For gas-phase polymerization, some authors have reported surface temperatures of such particles of up to $30^{\circ} \mathrm{C}$ higher than the temperature of the bulk phase [26]. A possible consequence of this "local overheating" can be a higher reaction rate at the beginning but a faster deactivation at the end - as shown in Figure 6.1.

As can be seen in Figure 6.1 for gas-phase polymerization, introducing hydrogen significantly decreases the polymerization rate, which confirms to other reported data (retarding effect; see Natta, Kissin, Zhakarov). In the presence of hydrogen, the rate peak is reached later. The reaction rate-profile without hydrogen rises faster and reaches the maximum within 5 minutes $\left(\mathrm{t}_{\max } \approx 5 \mathrm{~min}\right)$ followed by a rapid deactivation. The more hydrogen is used, the lower the rates of activation and deactivation. Although the activation time required for reaching the maximum rate does not change with increasing hydrogen partial pressure, it is still double $\left(\mathrm{t}_{\max } \approx 10 \mathrm{~min}\right)$ than in the absence of hydrogen. The lower the reaction rate, the lower the deactivation. Are temporarily deactivated sites (see equation 3.4) resistent to deactivation? That would explain both the lower rate and the increasing yield at longer reaction times caused by lower deactivation in the presence of hydrogen, as shown in Figure 6.1.

Contradictory to gas phase, the rate in slurry clearly increases in the presence of hydrogen; see Figure 6.2, and one can see that hydrogen reduces the activation period required to reach maximum activity in slurry phase, and the shift of the peak position is the opposite of that seen in the gas-phase. The higher the hydrogen partial pressure (in slurry) the shorter the activation period. 


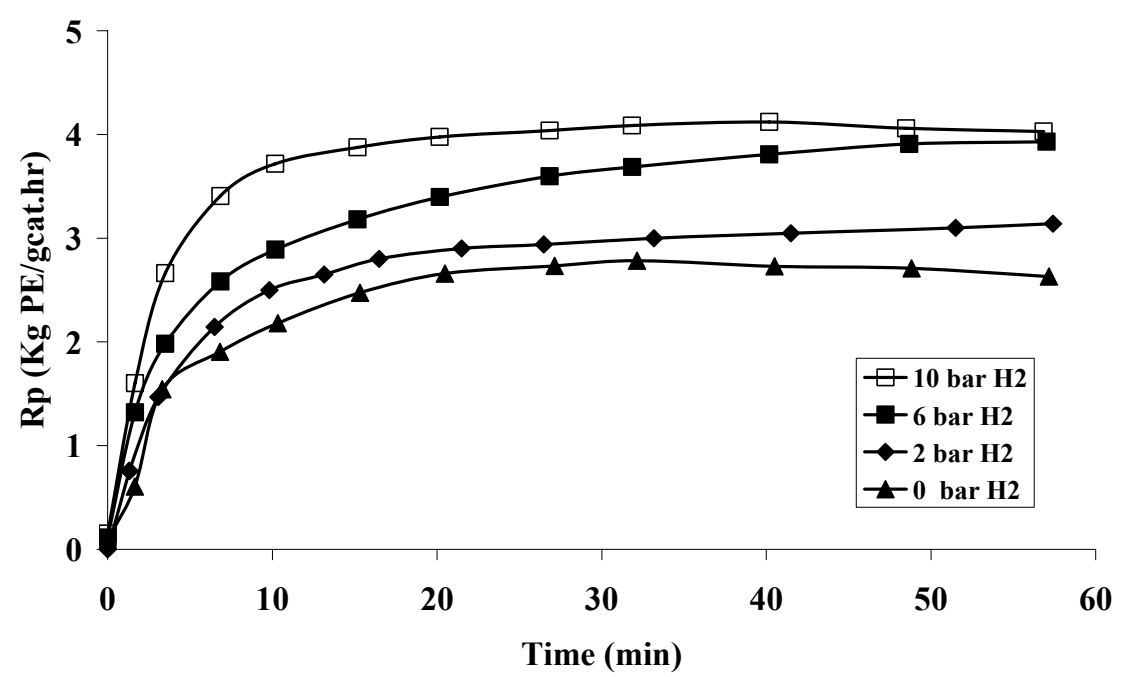

Figure 6.2-Hydrogen influence on reaction rate in slurry polymerization at $\mathrm{T}=80^{\circ} \mathrm{C}$ and $\mathrm{P}_{\mathrm{C} 2}=2 \mathrm{bar}$

This opposite effect in slurry polymerization contradicts Kissin's finding [18, 76], see above, but a clear explanation can be found by analyzing the fragmentation behaviour of the particles as described in the GRAF hypothesis:

\subsubsection{Morphology}

With increasing hydrogen pressure, a clear shift to smaller size of particles for all particles can be seen in Figure 6.3 and Figure 6.4 in both slurry and gas-phase homoethylene polymerizations.

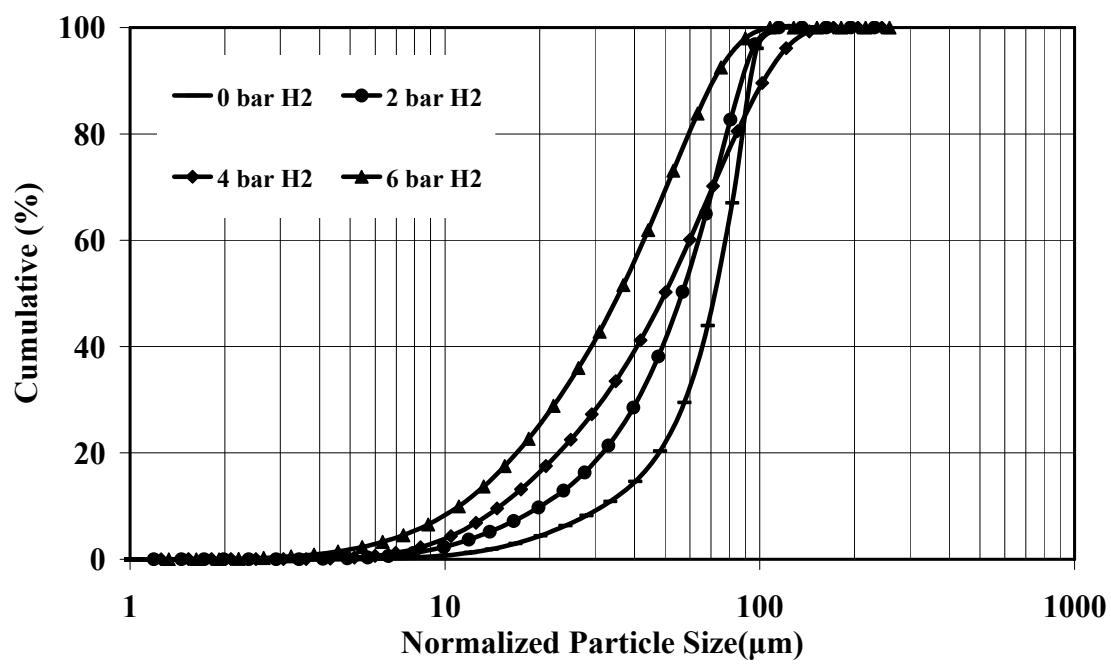

Figure 6.3-Hydrogen influence on cumulative PSD profiles normalized with the yield $\left(\mathrm{T}=80^{\circ} \mathrm{C}\right.$ and $\mathbf{P}_{\mathrm{C} 2}=\mathbf{2}$ bars.) in gas-phase ethylene polymerization $\square \mathbf{P}_{\mathrm{H} 2}=6 \mathrm{bar}, \square \mathbf{P}_{\mathrm{H} 2}=4 \mathrm{bar}, \triangle \mathbf{P}_{\mathrm{H} 2}=2 \mathrm{bar}$, $\mathbf{P}_{\mathrm{H} 2}=\mathbf{0 b a r}$

These PSDs indicate fines generation in both cases, but this effect is much stronger in slurry. We conclude that the brittleness of the growing particles is higher in slurry 
polymerizations, leading to particle fragmentation and disintegration; this disintegration in turn causes fines formation and increases the polymerization rate by formation and activation of new sites. This is the dominant effect in slurry polymerization - the polymerization rate increases with increasing hydrogen pressure. This effect happens at the beginning of the polymerization - see chapter 7 - therefore: the combination of growth stress and brittleness decides the question whether one finds rate acceleration or rate depression by hydrogen!

In gas-phase polymerization, the disintegration and generation of new active sites is more limited than in slurry. The polymerization-rate-decreasing chemical effect of hydrogen is dominant and consequently the rate decreases with increasing hydrogen pressure.

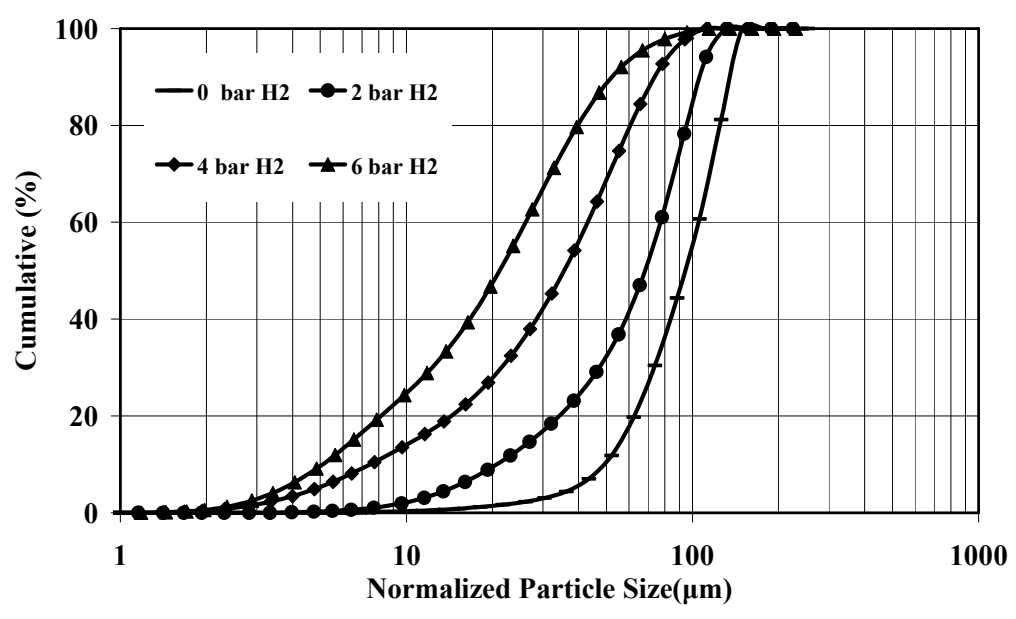

Figure 6.4-Hydrogen influence on cumulative PSD profiles normalized with the yield $\left(\mathrm{T}=\mathbf{8 0}^{\circ} \mathrm{C}\right.$ and $\mathbf{P}_{\mathrm{C} 2}=\mathbf{2}$ bars.) in slurry ethylene polymerization

A direct comparison of the PSD is given below. Figure 6.5 presents the clear evidence that fines generation in slurry polymerization due to the hydrogen is much more pronounced than fines generation in the gas-phase polymerization.

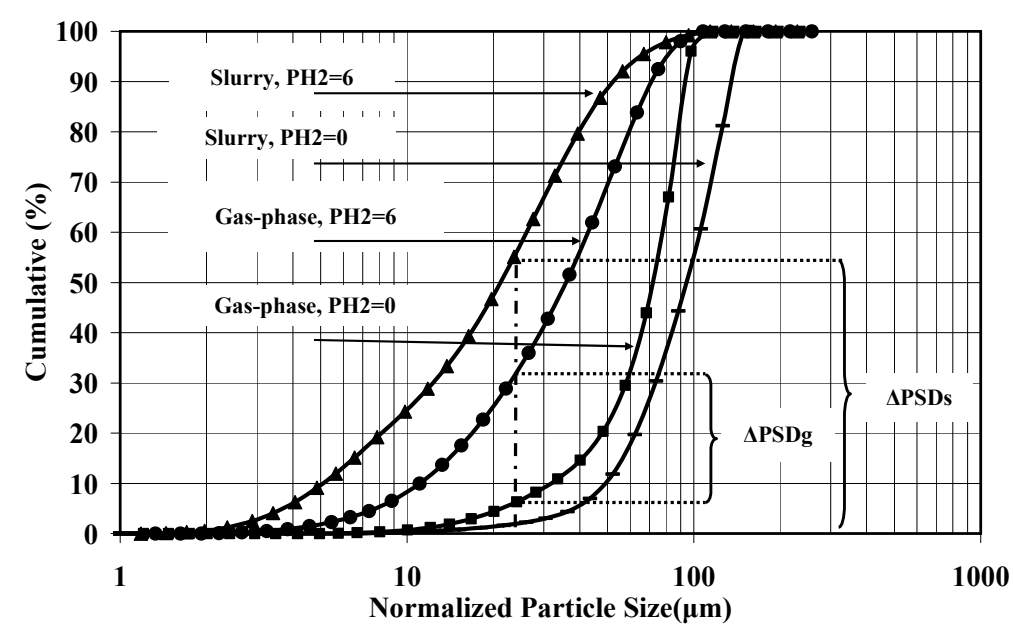

Figure 6.5-Hydrogen influence on cumulative PSD profiles normalized with the yield on slurry and gas-phase ethylene polymerization at $\mathrm{T}=80^{\circ} \mathrm{C}$ and $\mathrm{P}_{\mathrm{C} 2}=2$ bars. 
Figure 6.6 shows the different effect of hydrogen on the polymerization rate in slurry and gasphase polymerizations carried out under comparable conditions - which is now, based on GRAF fully understandable ${ }^{1}$ :

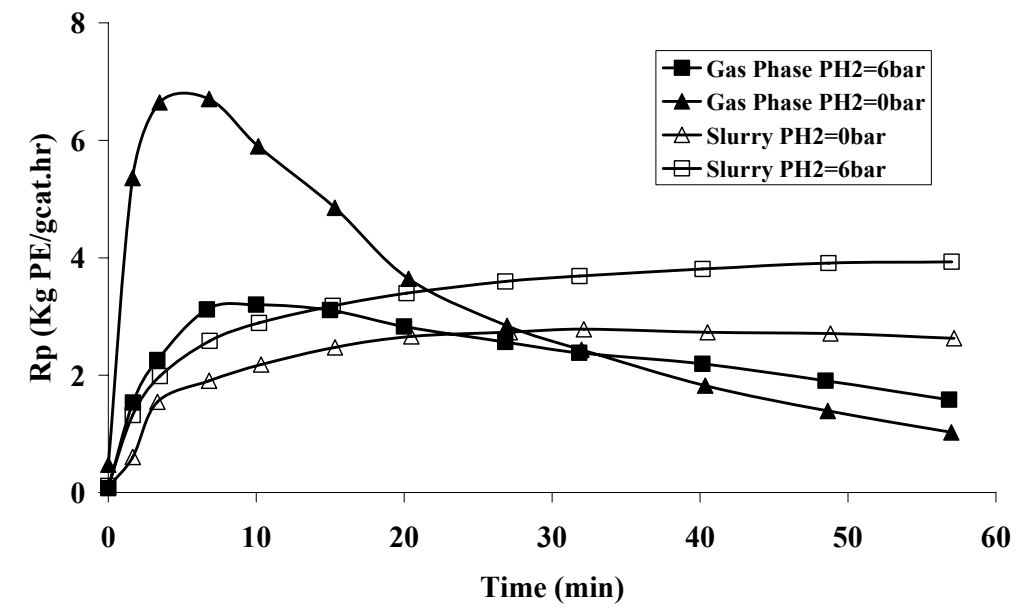

Figure 6.6-Hydrogen Influence on Slurry and Gas-phase Polymerization at $\mathrm{T}=80^{\circ} \mathrm{C}$ and $\mathrm{P}_{\mathrm{C} 2}=2$ bars.

Figure 6.7 and Figure 6.8 show the SEM images of the polymers produced in gasphase, with and without hydrogen respectively. Large cracks in the surface with multigrain structures inside the polymer particle are clearly visible for the sample produced at a higher hydrogen pressure. It seems that expansion of the solid phase under high growth stress dominates the morphology of the produced polymer, whereas for the polymer produced at a high hydrogen pressure, a high level of brittleness mainly controls the morphology: the particles break at lower stress levels (at lower energy accumulation) and therefore the polymer does not suffer from high stress. This is similar to an earthquake: higher brittleness leads more quickly to relaxation and avoids energy accumulation this way.

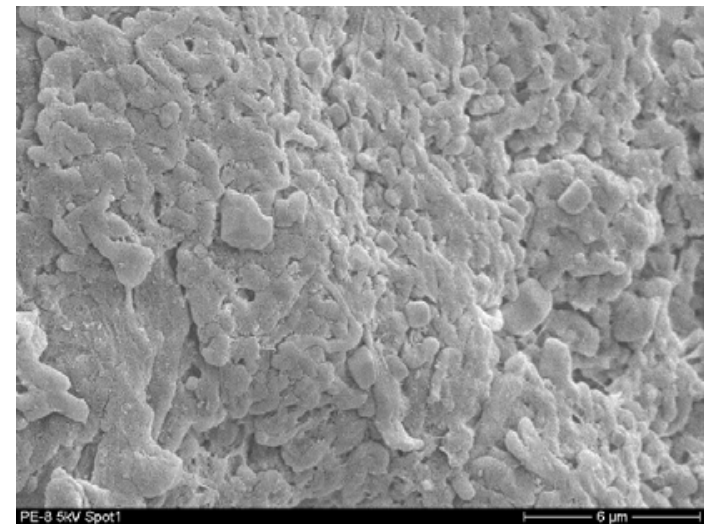

A

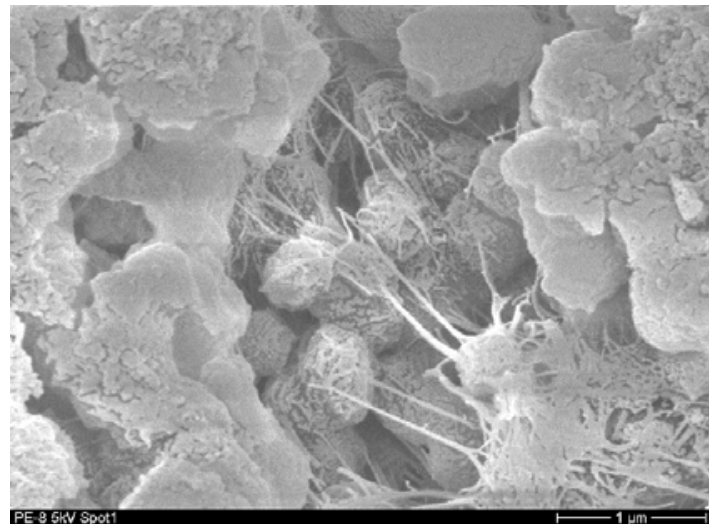

$\mathrm{B}$

Figure 6.7-The SEM images, Gas phase $P_{\mathrm{H} 2}=0$ bar (A) at a $6 \mu \mathrm{m}$ resolution (B) at a $1 \mu \mathrm{m}$ resolution

${ }^{1}$... and cannot be explained based on chemical effects 


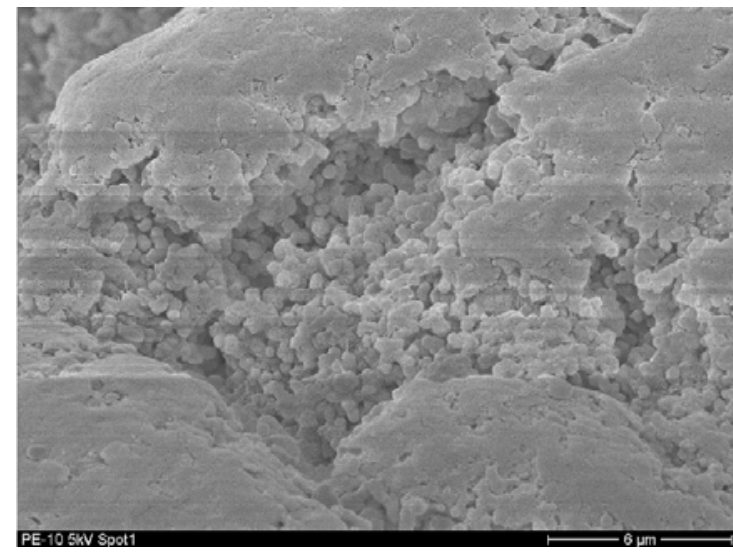

A

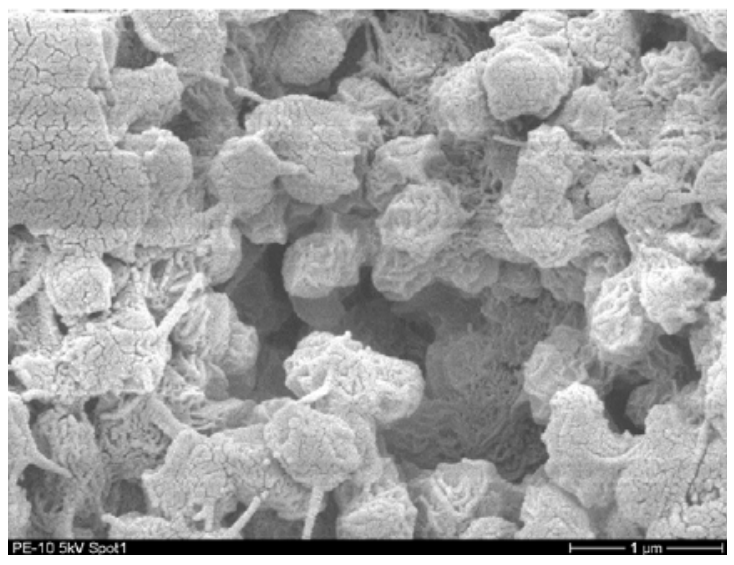

B

Figure 6.8-The SEM images, Gas-phase $P_{\mathrm{H} 2}=6$ bar $(\mathrm{A})$ at a $6 \mu \mathrm{m}$ resolution (B) at a $1 \mu \mathrm{m}$ resolution

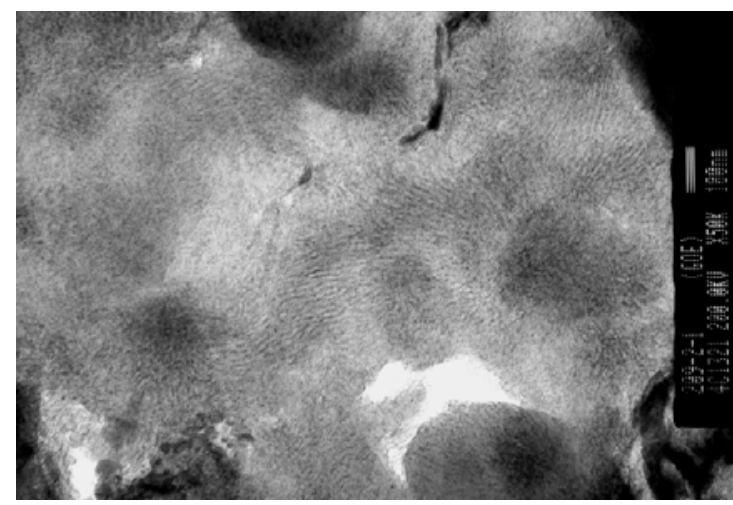

A

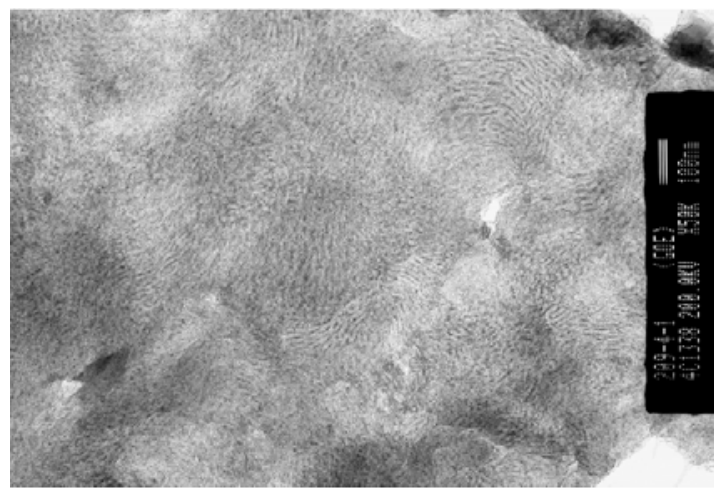

B

Figure 6.9-The TEM images of the polymer obtained during run (A) gas-phase $P_{\mathrm{H}_{2}}=0$ bar (B) gas-phase $\mathbf{P}_{\mathrm{H} 2}=6$ bar

The TEM images of the mentioned gas-phase polymers are shown in Figure 6.9. As can be seen, larger crystallites are formed at a higher hydrogen pressure.

Figure 6.10 and Figure 6.11 show the SEM images of the polymers produced in slurry, with and without hydrogen respectively. In the absence of hydrogen, Figure 6.10,

- the surface of the particles is to a large extent "open"

- many pores are visible

- fibrillar structures as an indication of the expansion of the solid phase under growth stress.

However, the SEM images of the polymer produced at $80^{\circ} \mathrm{C}$ in the presence of hydrogen $\left(\mathrm{P}_{\mathrm{H} 2}=6\right.$ bar) (see Figure 6.11) show more external and internal cracks in the whole structure, which is similar to clay structures. This is a clear evidence of a higher brittleness resulting from a higher crystallinity obtained (80\%). The higher reaction rate observed for the polymer shown in Figure 6.6 can be explained by a larger surface and more accessible active centres. 


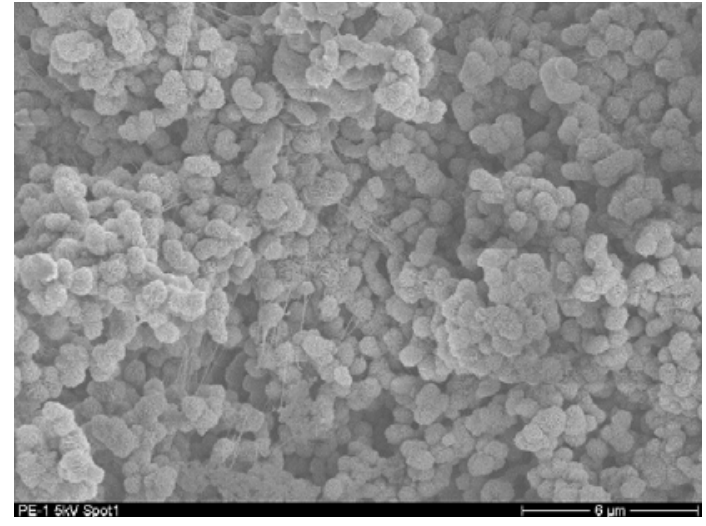

A

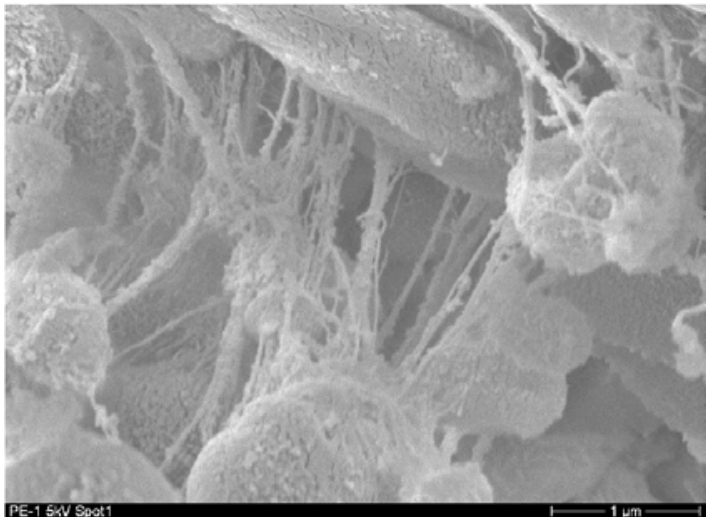

$\mathrm{B}$

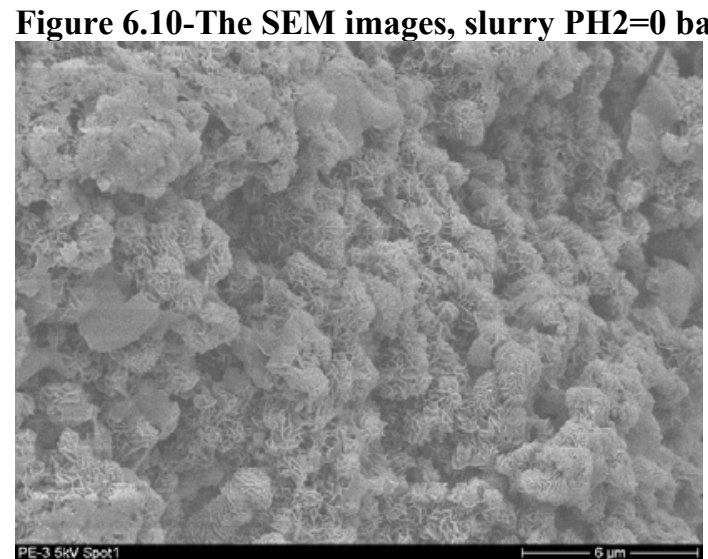

A

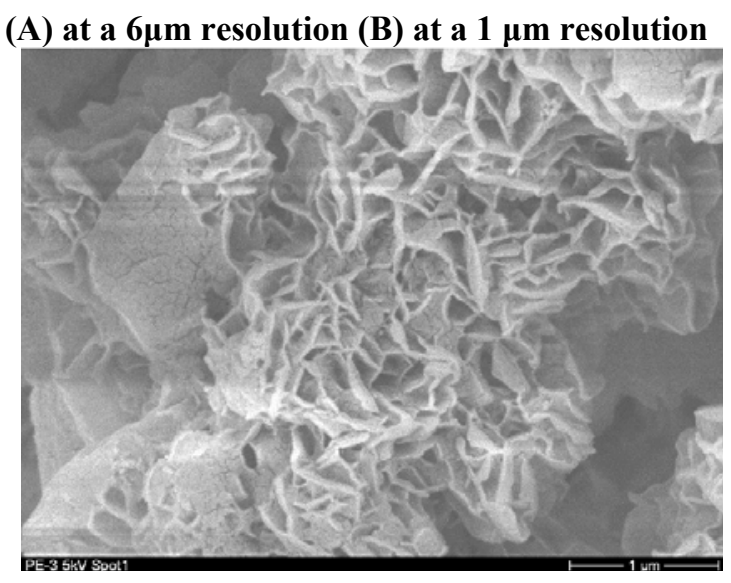

B

Figure 6.11-The SEM images, slurry $P_{\mathrm{H}_{2}}=6$ bar (A) at a $6 \mu \mathrm{m}$ resolution (B) at a $1 \mu \mathrm{m}$ resolution The TEM images of the polymer samples produced with and without hydrogen at $80^{\circ} \mathrm{C}$ are shown in Figure 6.12. Clearly, in the absence of hydrogen, it shows

- uniform distributed crystalline regions and amorphous regions in the whole structure.

- the crystal size was relatively small.

However, the TEM electron microscopy of the sample produces in the presence of hydrogen, $\mathrm{P}_{\mathrm{H} 2}=6$ bar, reveals a larger crystal size compared to sample produces in the absence of hydrogen. It seems that improved chain mobility - due to smaller chains and solvent participation - helps to generate larger crystals.

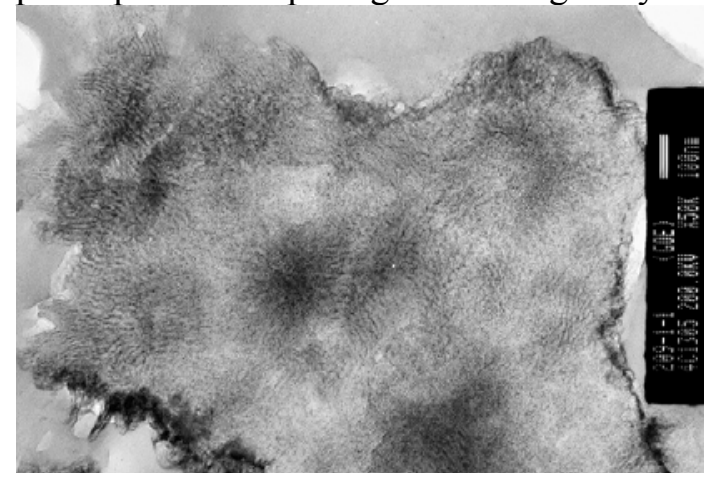

A

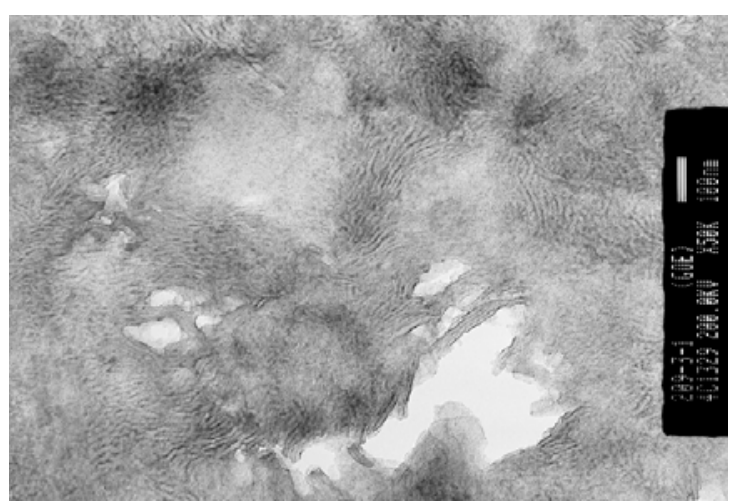

B

Figure 6.12-The TEM images of the polymer obtained during run (A) slurry $P_{\mathrm{H}_{2}}=0$ bar (B) slurry $\mathbf{P}_{\mathrm{H} 2}=6$ bar 
Does the analysis of the polymer crystallinity support the findings above? Does the particle disintegration correlate with the crystallinity under reaction conditions given in this chapter? This can be answered by analyzing the DSC results:

\subsubsection{Crystallinity}

The $1^{\text {st }}$ heating curves occurred at $10^{\circ} \mathrm{C} / \mathrm{min}$, and are separately shown in Figure 6.13 and Figure 6.14 for slurry and gas-phase samples respectively. Increasing the hydrogen partial pressure in the reaction medium of both phases accompanied by decreasing molecular weight and:

- $\quad$ shifts the curve to lower temperatures, i.e. a lower melting point

- increases the absolute magnitude, i.e. increasing the crystallinity accompanied by decreasing crystal size

- decreases the broadness of the peaks, i.e. a more uniform crystal size distribution.

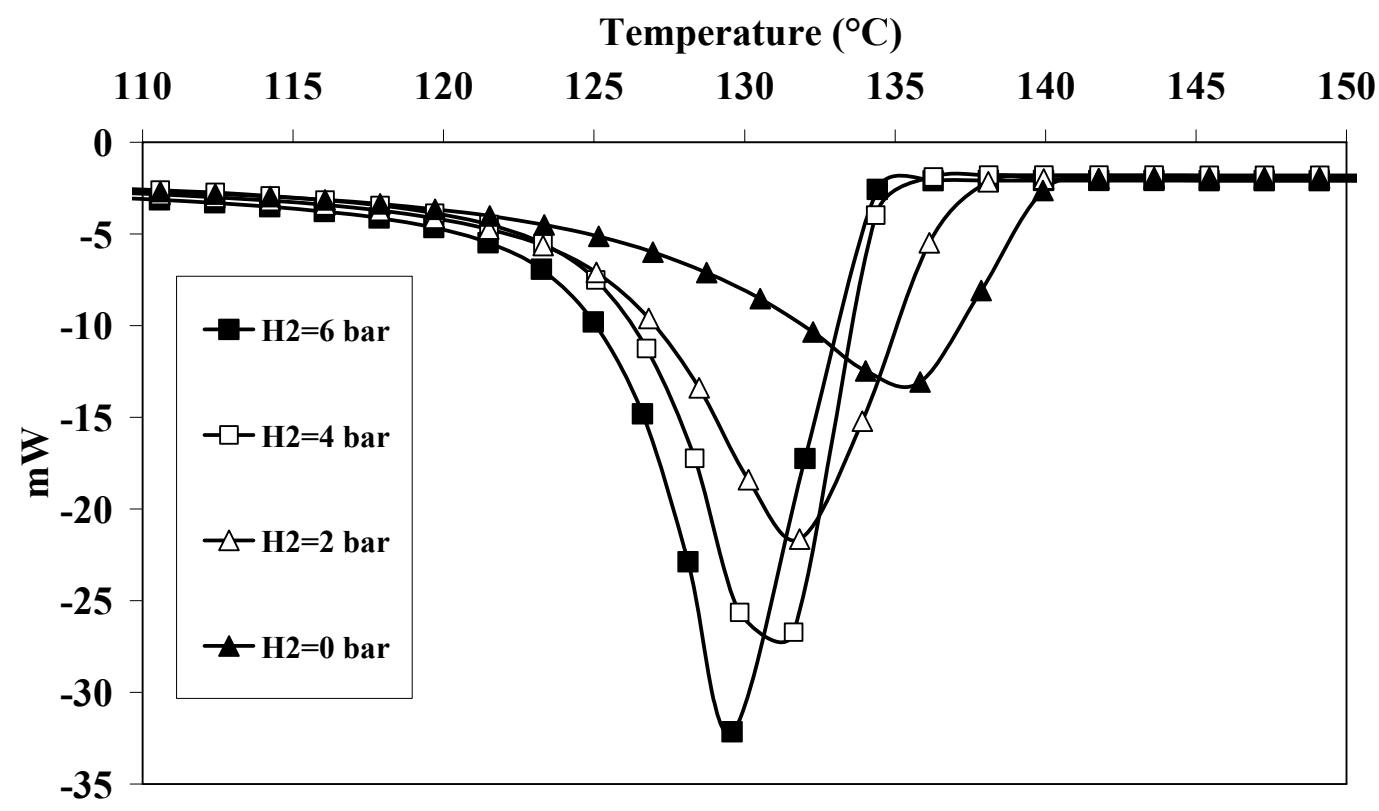

Figure 6.13-DSC scan for HDPE samples obtained in gas-phase ethylene polymerization (1st heating, heating rate: $10 \mathrm{C} / \mathrm{min}$, N2 Atmosphere) 


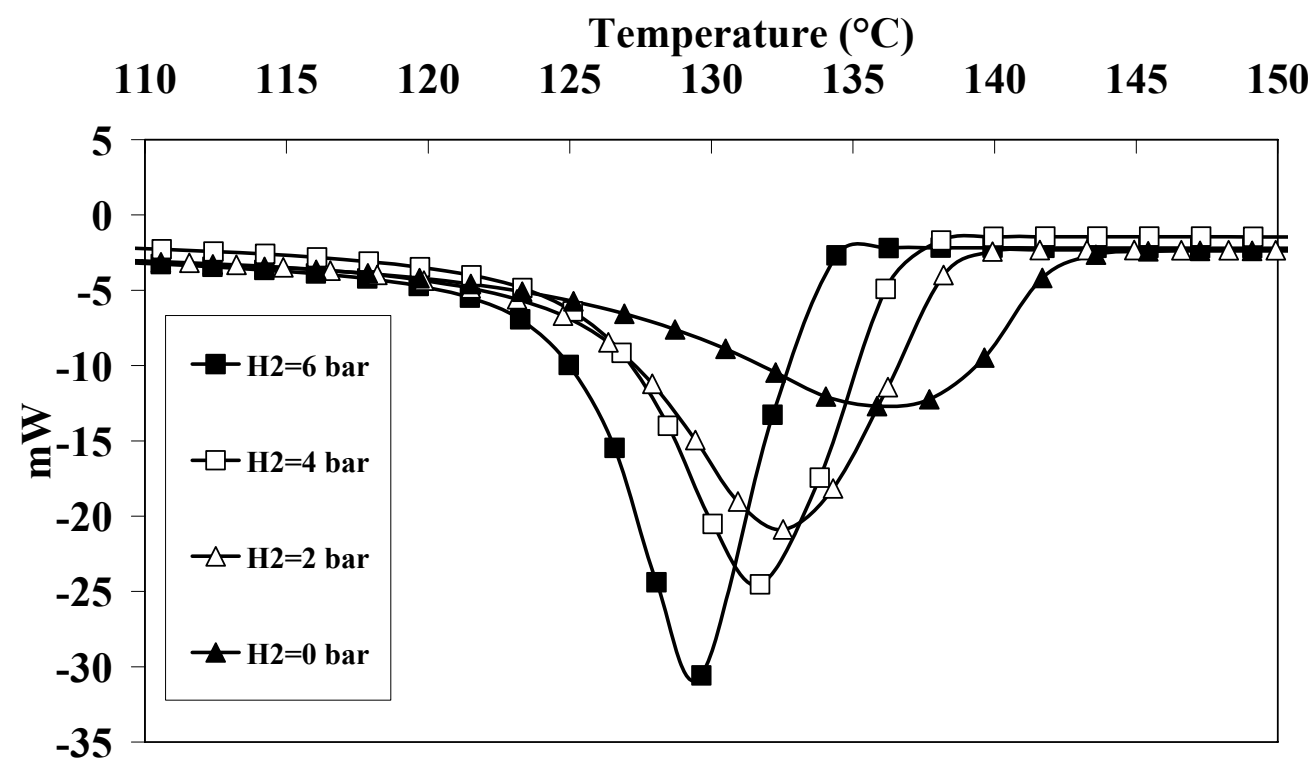

Figure 6.14-DSC scan for HDPE samples obtained in slurry ethylene polymerization (1st heating, heating rate: $10 \mathrm{C} / \mathrm{min}$, N2 Atmosphere)

The degree of crystallinity for each polymer sample in the slurry- and gas-phase experiments is indicated in Table 6.1 and shown in Figure 6.15. Starting at 55..60\% in the absence of hydrogen, the maximum (plateau) is reached at about $78 . .80 \%$ at $\mathrm{P}_{\mathrm{H} 2}=5$ bar. $\mathrm{X}_{\mathrm{C} 1}$ is higher in slurry polymerizations before the plateau is reached - the higher mobility of chains in the hexane swollen amorphous PE is assumed to be the reason.

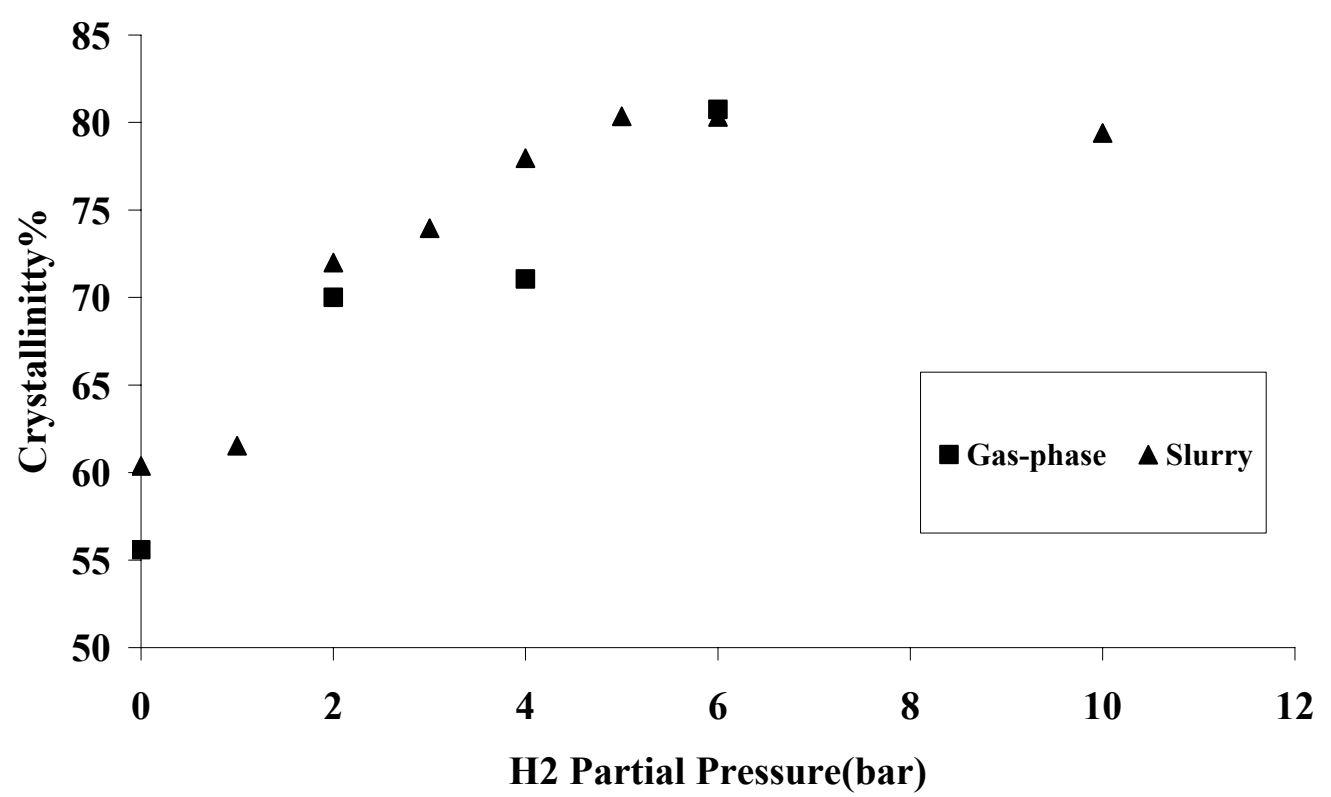

Figure 6.15-Comparison between the $1^{\text {st }}$ crystallinity of polymer produced in gas-phase and slurry ethylene polymerization at various hydrogen partial pressures 
Figure 6.16 shows the effect of hydrogen on the melting point, which shows that: the melting temperature decreases with increasing crystallinity but levels out at about 5 bar hydrogen partial pressure.

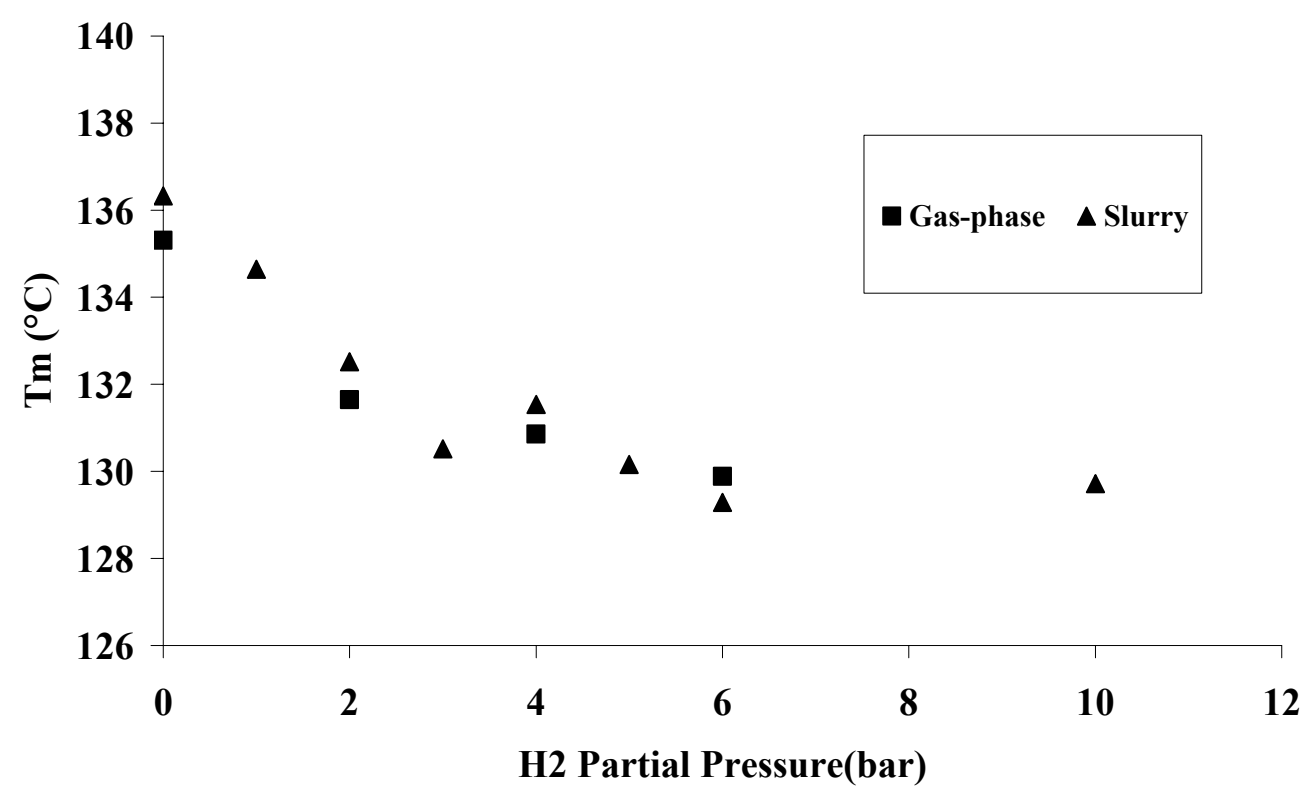

Figure 6.16-Comparison of Tm of polymer produced in gas-phase and slurry homo-ethylene polymerization at various hydrogen partial pressures

Following GRAF, the next issue to be discussed, must be the molecular weight: It is well known that the shorter chains (higher chain mobility) form crystals faster and the crystallization degree is higher. Therefore, the analysis of the MWD can provide a helpful support of the GRAF hypothesis:

\subsubsection{Molecular Weight}

Table 6.1 summarizes the operating conditions and some important properties of polymer produced in hydrogen experiments in slurry and gas-phase. By increasing the hydrogen partial pressure in both phases, as expected and reported by other research studies, the clear decrease in weight average molecular weight and the number average molecular weight can also be seen in our results as shown in Table 6.1.

By increasing the partial pressure of hydrogen from 0 to 6 bar in both phases, the decreasing response in $\mathrm{Mw}$ and $\mathrm{Mn}$ is more pronounced in the gas-phase (15-fold and 24fold decreasing, respectively) than in the slurry phase (decreasing around 8-fold and 18-fold, respectively). This can be attributed to the lower concentration of hydrogen in slurry. Both phases show higher dependency in decreasing in Mn (a 24-fold decrease for gas-phase and an 18-fold decrease for slurry) compared to $\mathrm{Mw}$ (a 15-fold for gas-phase and an 8-fold times for slurry) which is another expression for increasing the broadness of molecular weight by hydrogen. 
Table 6.1-Molecular weight characteristics, crystallinity and melting temperature

\begin{tabular}{lccccccccc}
\hline Run & $\begin{array}{c}\mathrm{P}_{\mathrm{H} 2} \\
(\mathrm{bar})\end{array}$ & $\begin{array}{c}\mathrm{C}_{\mathrm{H} 2} \\
(\mathrm{~g} / \mathrm{L})\end{array}$ & $1000 \frac{C_{\mathrm{H}_{2}}}{C_{C_{2}}}$ & $\begin{array}{c}\mathrm{Mw} \\
(\mathrm{kg} / \mathrm{mol})\end{array}$ & $\begin{array}{c}\mathrm{Mn} \\
(\mathrm{kg} / \mathrm{mol})\end{array}$ & $\begin{array}{c}\mathrm{Mw} / \mathrm{Mn} \\
\mathrm{X}_{\mathrm{C} 1} \\
\%\end{array}$ & $\begin{array}{c}\mathrm{X}_{\mathrm{C} 2} \\
\%\end{array}$ & $\begin{array}{c}\mathrm{Tm} \\
\left({ }^{\circ} \mathrm{C}\right)\end{array}$ \\
\hline 1-Gas & 0 & 0 & 0 & 688 & 163 & 4.2 & 65 & 56 & 135.1 \\
2-Gas & 2 & 0.137 & 72 & 144 & 13 & 11 & 67 & 70 & 131.7 \\
3-Gas & 4 & 0.274 & 140 & 90 & 9 & 10 & 66 & 71 & 130.9 \\
4-Gas & 6 & 0.411 & 220 & 47 & 6.9 & 6.8 & 75 & 81 & 129.9 \\
\hline 5-Slurry & 0 & 0 & 0 & 560 & 114 & 4.9 & 67 & 60 & 136.3 \\
6-Slurry & 2 & 0.027 & 5.2 & 140 & 15 & 9.2 & 69 & 73 & 132.5 \\
7-Slurry & 6 & 0.088 & 17 & 71 & 6.2 & 11.4 & 75 & 80 & 129.3 \\
8-Slurry & 10 & 0.148 & 29 & 46 & 5.2 & 8.9 & 74 & 79 & 129.7 \\
\hline
\end{tabular}

As can be seen, with the same hydrogen partial pressure in both slurry and gas-phase experiments, due to the solubility effect the hydrogen concentration and hydrogen -toethylene ratio around the growing particles in slurry is nearly 5-fold and 15-fold less respectively than its concentration in the gas-phase. However, this huge difference is not matched by the same response in molecular weight - the molecular weights of polymer produced under similar conditions in gas and slurry is nearly the same.

If the active sites were in direct contact with the bulk, the molecular weights in the gas-phase would be much lower than in slurry under the conditions applied here. This is clearly not the case. Under isothermal conditions, two explanations are possible if we retain to the same mechanisms:

- $\mathrm{H}_{2}$ enrichment near the active site in slurry ${ }^{1}$ or

- Decrease of $\mathrm{C}_{2}$ concentration near the active site in slurry

Either explanation might be because of the very different sorption and transport mechanisms for hydrogen and ethylene in gas and slurry, if one takes into account the polymer layer around every active site through which all components have to diffuse.

It is clearly evident from Table 6.1 that hydrogen shows a strong impact on PDI. This can be caused by the hydrogen influence on propagation (Kissin 2001-1999) or on chaintransfer [92]. This can be explained as a consequence of the different hydrogen response of different active sites.

Table 6.1 presents the expected increase in crystallinity with increasing hydrogen partial pressure. $\mathrm{X}_{\mathrm{C} 2}$ is higher than $\mathrm{X}_{\mathrm{C} 1}$ in all samples except the slurry sample produced in the absence of hydrogen. Clearly, long molecules produced in-situ are forced to build more parallel structures than under melting-recrystallization conditions ${ }^{2}$, whereas melting and recrystallization increases the folding ability of chains in all other cases.

\footnotetext{
${ }^{1}$ as described in chapter 5 , the ethylene solubility can change the $\mathrm{H}_{2}$ solubility - the more ethylene solved the more hydrogen is present

${ }^{2}$ where they are probably hindered by the entanglement
} 


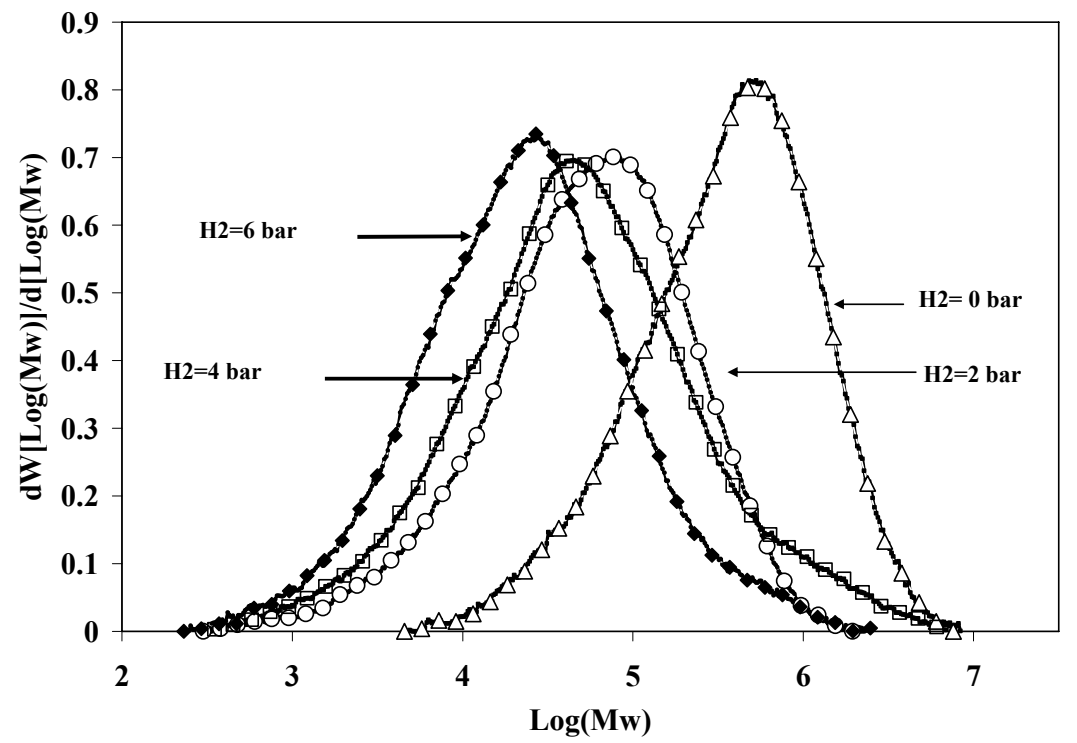

Figure 6.17-Hydrogen influence on MWDs of polyethylene produced in gas-phase polymerization at $\mathrm{T}=80^{\circ} \mathrm{C}$ and $\mathrm{P}_{\mathrm{C} 2}=\mathbf{2}$ bars.

The MWDs of polyethylene produced in hydrogen series slurry and gas-phase experiments are shown in Figure 6.17 and Figure 6.18 respectively. As can be seen, by increasing the hydrogen partial pressure in the reaction medium, the molecular weight curves shift significantly to the left towards the low molecular weight region by keeping a clear shoulder in the high molecular weight region. This shoulder is more pronounced for slurry polymerization. Lower hydrogen concentration in slurry and the existence of at least one type of active centres in the catalyst structure on which hydrogen has less influence compared to other centres could be the cause of this finding.

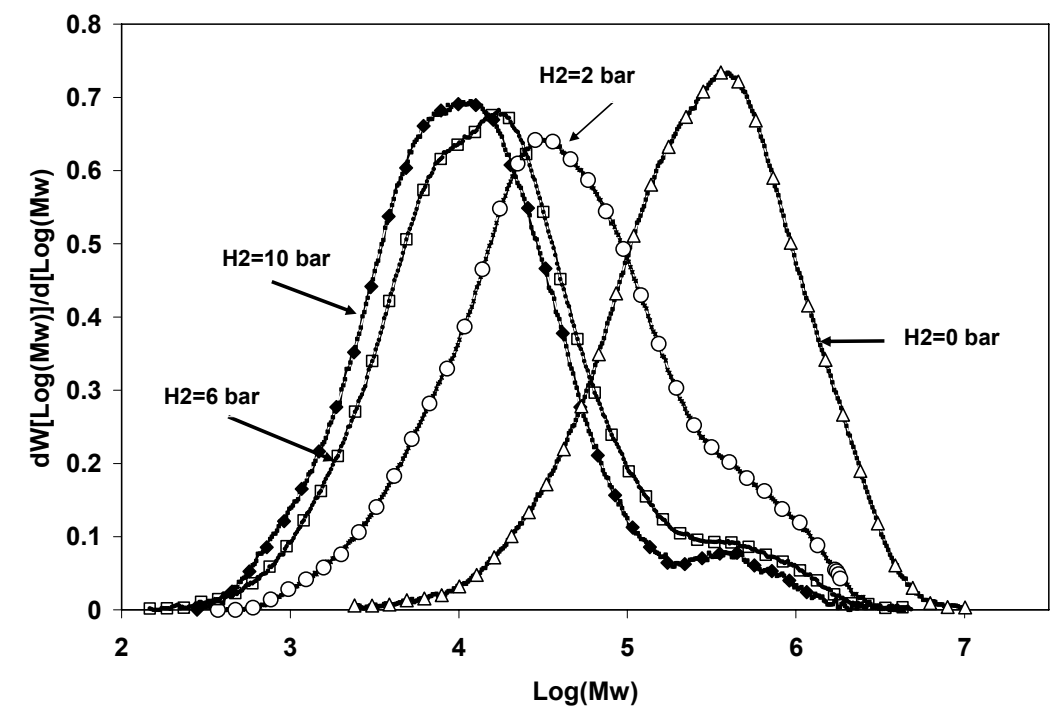

Figure 6.18-Hydrogen influence on MWDs of polyethylene produced in slurry polymerization at $\mathrm{T}=80^{\circ} \mathrm{C}$ and $\mathrm{P}_{\mathrm{C} 2}=\mathbf{2}$ bars.

By comparing Figure 6.1, Figure 6.2, Figure 6.17 and Figure 6.18, one can see that the lowering molecular weight by introducing hydrogen is virtually independent of the shape of kinetic curves either build-up curve (Figure 6.1 for gas-phase) or decay type (Figure 6.2 
for slurry-phase) and is also independent of its rate effect, whether the retarding effect (gasphase) or the enhancement effect (slurry), which is expected.

Figure 6.19 shows MWD of four samples obtained in slurry and gas-phase ethylene polymerization with ( $\mathrm{PH} 2=6$ bars) and in the absence of hydrogen. As can be seen, the slurry samples have lower peaks and shift a little towards a low molecular weight. Due to the higher local reaction temperature at the particle level of gas-phase ethylene polymerization compared to slurry, one might expect that more chains are terminated by transfer reactions in gas phase leading to lower molecular weight. However, the trend in Figure 6.19 shows that this interpretation is not true - it was found earlier that the initial phase of overheating clearly has little impact on the final MWD. We assume more chain transfer is initiated by solvent or cocatalyst in slurry phase.

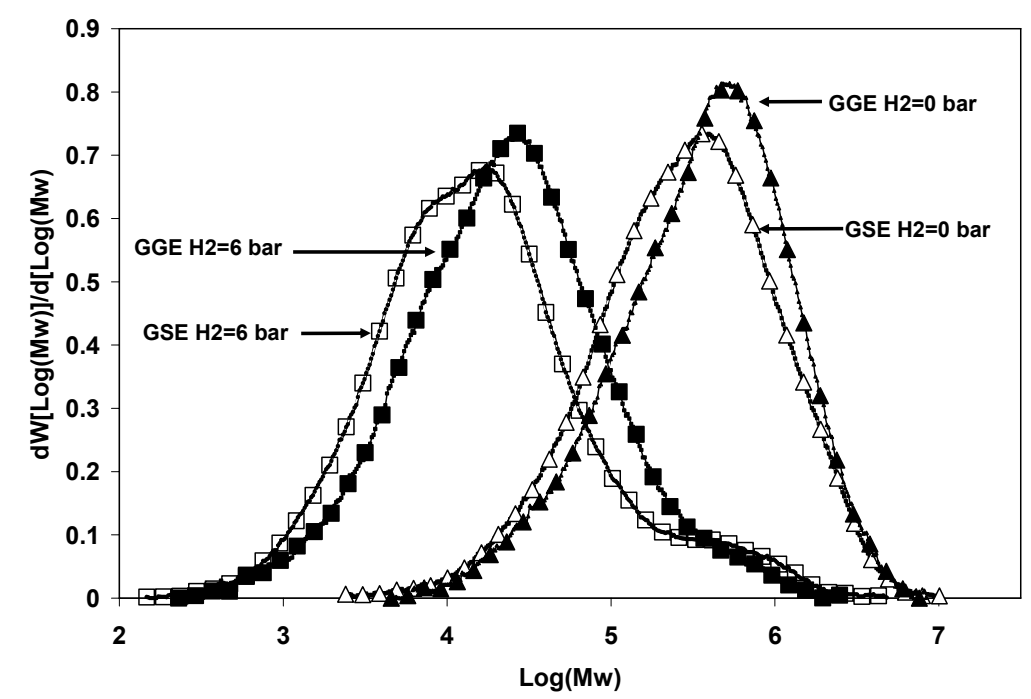

Figure 6.19-Hydrogen influence on MWDs of polyethylene produced in slurry and gas-phase polymerization at $\mathrm{T}=80^{\circ} \mathrm{C}$ and $\mathrm{P}_{\mathrm{C} 2}=2$ bars.

The MWDs of polyethylene produced in slurry and gas-phase polymerization at the same hydrogen partial pressure differ little if one takes into account that the concentration ratio of hydrogen to ethylene in the bulk is about 13 times lower in slurry. Therefore, we have good reasons to use the partial pressure in our modelling equations rather than the concentrations.

Plotting the inverse molecular weight versus the hydrogen partial pressure shows a linear (first order regarding the hydrogen partial pressure) function similar to that which one would expect in the case of a single site catalyst; see Figure 6.20 and equation 6.10. It is very surprising that this function expresses both gas phase and slurry results:

$$
100,000 / M_{W}=3.255 P_{H 2}+0.6432 \quad \text { Equation } 6.10
$$




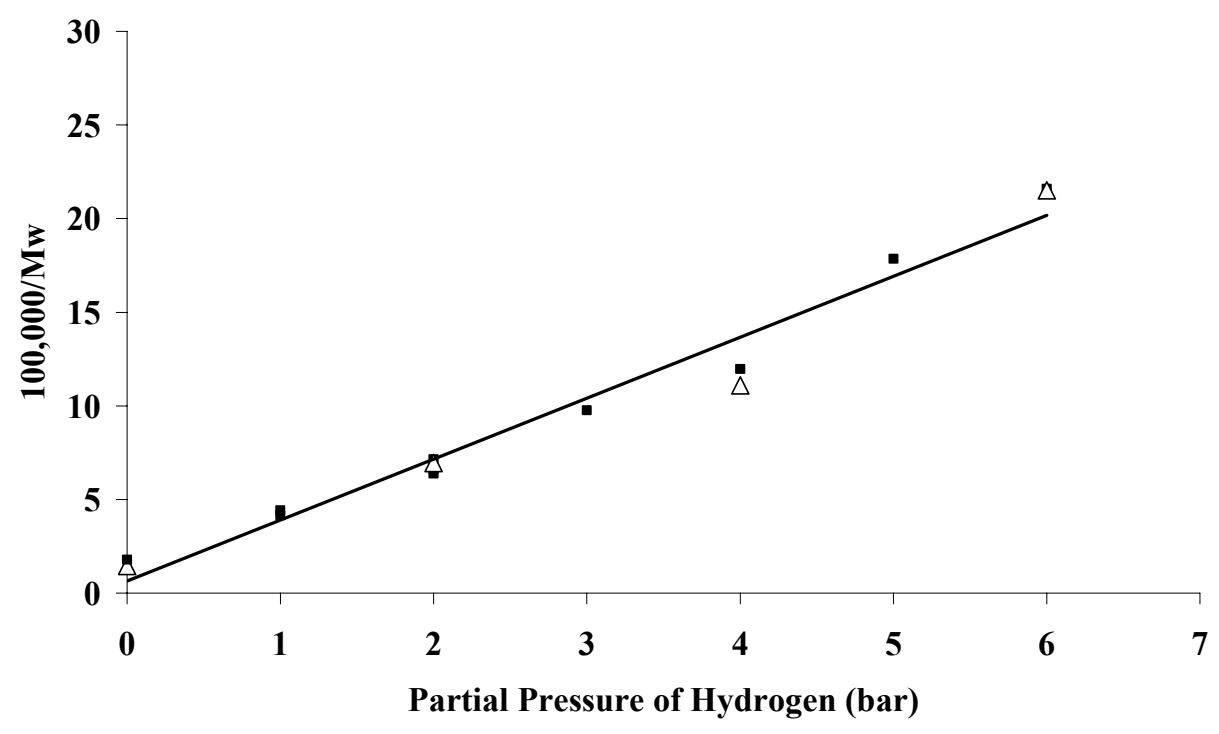

Figure 6.20-Curve fitting for obtaining the relation between hydrogen partial pressure and inverse of molecular weight for slurry and gas-phase at $T=80^{\circ} \mathrm{C}$ and $P_{C 2}=2$ bars, $\Delta \mathrm{Gas}$-phase, slurry

However, the used Z-N catalyst consists of different sites - how does it look like for the contribution of different active sites? Deconvolution of the MWD into Flory components as described in Chapter 2 was performed.

Table 6.2-Hydrogen influence on parameters obtained by deconvolution method

\begin{tabular}{cccccc}
\hline & & \multicolumn{2}{c}{ Slurry } & \multicolumn{2}{c}{ Gas-phase } \\
\cline { 2 - 6 }$P_{H_{2}}$ bar & Site No & $\% m_{j}$ & $\begin{array}{c}\text { Mw/1000 } \\
(\mathrm{g} / \mathrm{gmol})\end{array}$ & $\% m_{j}$ & $\begin{array}{c}\text { Mw/1000 } \\
(\mathrm{g} / \mathrm{gmole})\end{array}$ \\
\hline 0 & 1 & 3.1 & 14 & 0 & - \\
& 2 & 21.9 & 54 & 14 & 47.1 \\
& 3 & 41.4 & 170 & 26.8 & 169 \\
& 4 & 20.3 & 418 & 41.6 & 358 \\
& 5 & 13.3 & 820 & 17.6 & 848 \\
\hline 2 & 1 & 8.1 & 2.6 & 5.9 & 2 \\
& 2 & 32.8 & 11 & 29.3 & 9.51 \\
& 3 & 33.4 & 33 & 35.6 & 30.4 \\
& 4 & 14.5 & 99 & 18.6 & 97.3 \\
& 5 & 11.2 & 362 & 10.6 & 373 \\
\hline 4 & 1 & 16.6 & 2.03 & 11 & 2.16 \\
& 2 & 38.4 & 7.7 & 38 & 9.21 \\
& 3 & 26.8 & 22.1 & 32.6 & 27.2 \\
& 4 & 10.7 & 67.4 & 12 & 81.5 \\
& 5 & 7.5 & 332 & 6.4 & 308 \\
\hline 6 & 1 & 23.7 & 2.4 & 16.3 & 2.13 \\
& 2 & 42 & 8 & 39 & 7.76 \\
& 3 & 21.5 & 22.6 & 27.2 & 19.3 \\
& 4 & 6.7 & 77.4 & 12 & 46.5 \\
& 5 & 6.3 & 321 & 5.5 & 173 \\
\hline
\end{tabular}


From Table 6.2, Figure 6.21 and Figure 6.22 following results can be deduced:

- All MWDs can be fitted by five Flory components, with the exception of polymer produced in gas-phase without hydrogen. In that case, the reaction rate more rapidly reached higher values than others (see Figure 6.1) and probably the overheating at the particle level deactivates the low-molecular-weight site.

- In both phases, increasing the hydrogen partial pressure increases the contribution of the first two sites responsible for producing low molecular weight. This is combined with the decreasing contribution of the previous two sites that are responsible for producing a high molecular weight.

- The influence of solvent on increasing the contribution of the first site, which is responsible for producing the lowest molecular weight, in all slurry polymerization (in the presence or absence of hydrogen) is remarkable.

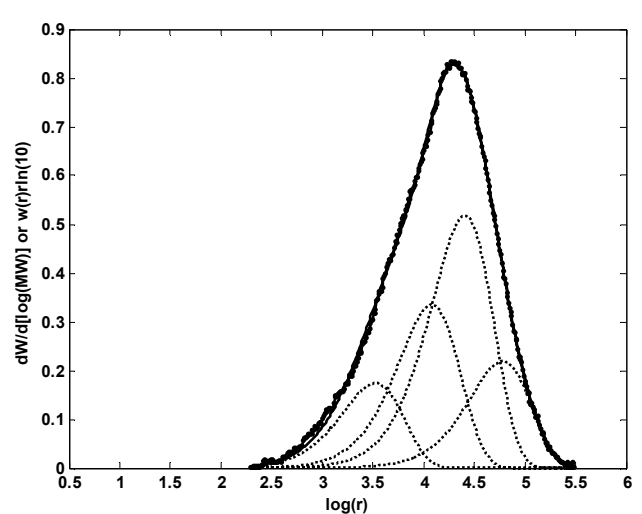

A) Gas-phase $\mathrm{P}_{\mathrm{H} 2}=0$ bar

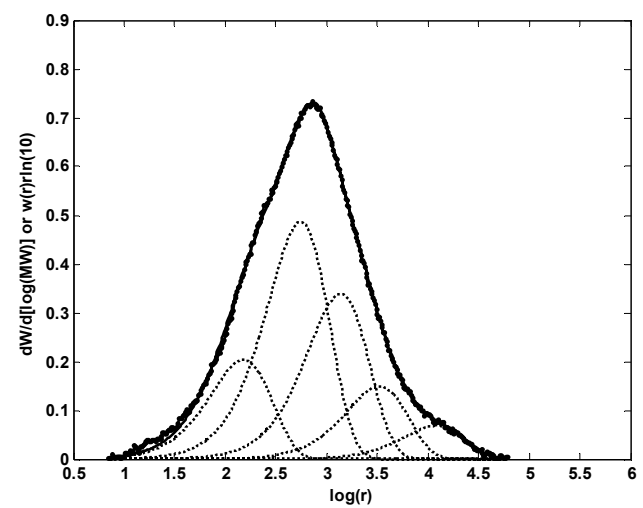

B) Gas-phase $\mathrm{P}_{\mathrm{H} 2}=6$ bar

Figure 6.21 -Comparison of deconvolution analysis at $\mathrm{PH2}=0$ bar and $\mathrm{PH} 2=6$ bar performed in gas-phase ethylene polymerization $\left(\mathrm{P}_{\mathrm{C} 2}=\mathbf{2}\right.$ bar $\left.\& \mathrm{~T}=80^{\circ} \mathrm{C}\right)$

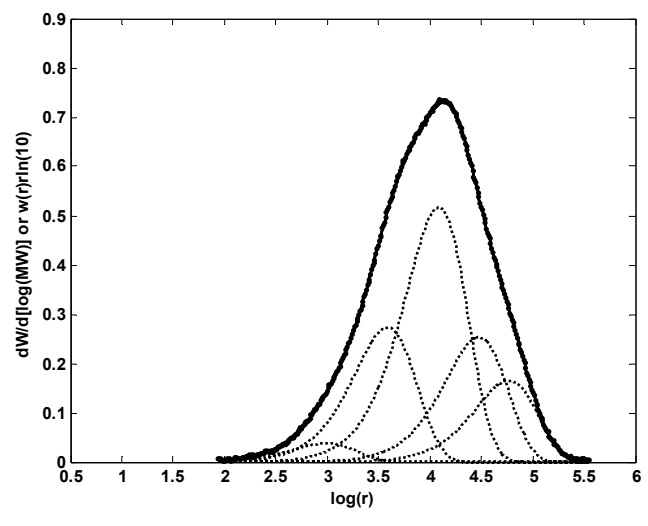

C) Slurry $\mathrm{P}_{\mathrm{H} 2}=0$ bar

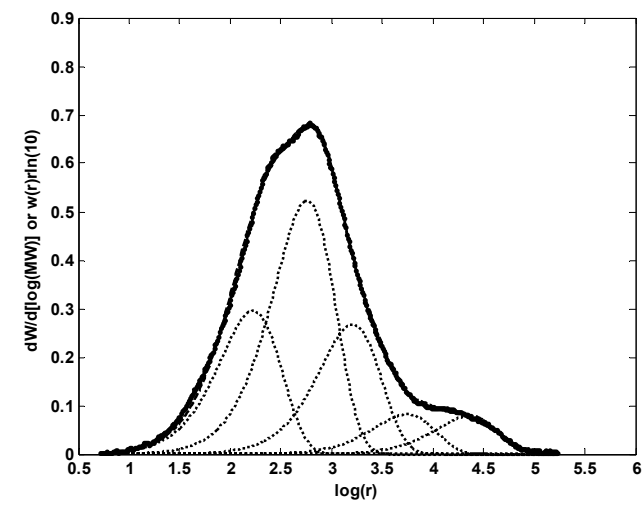

D) Slurry $\mathrm{P}_{\mathrm{H} 2}=6$ bar

Figure 6.22 -Comparison deconvolution analysis at $\mathbf{P}_{\mathrm{H} 2}=0$ bar and $\mathbf{P}_{\mathrm{H} 2}=6$ bar performed in slurry ethylene polymerization $\left(\mathrm{P}_{\mathrm{C} 2}=\mathbf{2}\right.$ bar $\left.\& \mathrm{~T}=80^{\circ} \mathrm{C}\right)$ 


\subsection{Conclusions}

The influences of hydrogen on polymerization kinetics and polymer microstructure characterization in gas-phase and slurry ethylene homo-polymerization was investigated resulting in the following statements:

In the gas-phase, the reaction rate decreases by increasing the hydrogen pressure; however, the opposite effect was seen in slurry phase.

The pressure of hydrogen shows a similarly strong effect on the molecular weight of the polymer produced in gas and slurry. Comparing the experimental results observed in the absence of hydrogen, we found slightly lower molecular weights in slurry compared to gasphase - clearly, there is a little more chain transfer in slurry, which can result from the hexane directly and/or from a higher contribution of the co-catalyst that reaches the active sites more easily in the presence of hexane.

DSC results confirm that hydrogen addition increases the level of crystallinity coupled with a simultaneous decrease in melting temperature.

Increasing the level of crystallinity can dramatically increase the production of fines in both phases and can change the particle size distribution accordingly if the brittleness of the more crystalline particles and the growth stress reach critical levels.

One can conclude that the polymer mobility, which can be influenced by many variables such as

- temperature,

- chain length of the polymer produced

- chain length of the dead polymer that surrounds the active sites,

- hexane content in the amorphous part of the polymer matrix that changes the microviscosity,

should be taken into account when interpreting the results. This different chain mobility leads to different in-situ crystallinity, which has a direct impact on the particle brittleness, and the particle can break at a critical growth stress that increases with the polymerization rate. It is now very clear that this effect can affect in slurry and gas-phase polymerization differently due to different sorption, swelling and micro conditions around the active centres.

Finally, this combination of the role of the polymer mobility as a physical effect with the chemical effect of retardation, previously reported by other researchers, can explain our new data and some peculiarities such as the increasing the rate of reaction in slurry ethylene polymerization which is caused by the disintegration of particles produced in the presence of hydrogen in both slurry and gas-phase ethylene polymerization. 



\section{Chapter 7}

\section{Two-Stage Polymerization}

\subsection{Introduction}

Multi-stage processes are common in polyolefin industries. Borstar (Borealis), CX (Mitsui), Hostalen (Lyondellbasell), and Spherilene (Lyondellbasell) polyethylene technologies are some examples [93]. However, this situation is not completely reflected by research activities, although there is increasing interest even within academic research. There are a number of significant reasons such as follows:

- improving the mechanical properties [94]

- increasing the yield of the catalyst $[30,95,96]$

- improving the morphology of the polymer $[29,95,97,98]$

- improving the rheological behaviour of the polymer [99]

- removing of sintering, agglomeration and fines generation [47].

Two-stage polymerization is typically used in industrial polymerizations by arranging two or more reactors in series $[100,101]$. Depending on the phase of the reaction, gas-phase or slurry, the first reactor operates either with a high hydrogen content for producing low molecular weight or in the absence of hydrogen to produce high molecular weight polymer. In the second reactor, the polymerization continues under different operating conditions; in particular, the hydrogen content is reversed (low or high) compared to the first reactor [102].

At lab scale, this can be simulated ${ }^{1}$ in one single reactor in such a way that after executing the first stage, the reaction is stopped by depressurizing and/or cooling, purging, and rapidly changing to new operating conditions. For example, bimodal MWD can be produced this way, see Figure 7.1.

To improve the morphology of the polymer and to reduce sintering, agglomeration and fines production, pre-polymerization is often an effective solution [103]. Prepolymerization is carried out at low yields under "mild" operating conditions such as low temperature, low monomer and low hydrogen pressure [97, 98, 104, 105]. During the prepolymerization, growing catalyst/polymer particles polymerize moderately uniformly and break through the early stage of polymerization under less stressing conditions due to lower growth stress and less overheating.

Little has been reported about the comparison of two-step polymerizations in gasphase and in slurry. This chapter describe four series of two-stage polymerizations that were

\footnotetext{
${ }^{1}$ The residence time distribution of a continuous (industrial) plant cannot be simulated in this way this is only possible by means of continuous (mini-) plants.
} 
performed to study and compare the impact of each step on the polymerization rate profiles, molecular weight, crystallinity and PSD of the powder produced using gas-phase catalyst, $\mathrm{C}_{\mathrm{g}}$.

In the first series (see Table 7.1), we performed 4 two-step slurry experiments in which the first step was executed in the absence of hydrogen ("low brittleness"). The overall reaction time was kept constant in all experiments at 1 hour; the ethylene pressure was constant at 2 bar. After a certain time $(0,6,12,20$ minutes $), 6$ bar hydrogen was added (leading to highly brittle PE) while keeping all other parameters constant ${ }^{1}$. Another experiment was carried out (for comparison) without any hydrogen addition, i.e. a 60 min run time at zero hydrogen, i.e. "low brittleness" because of high Mw, so that ductile PE is formed. From Chapter 6, it is clear that the catalyst disintegrates quickly if hydrogen is present from the beginning, but it was not clear how hydrogen acts after some ductile PE had been formed during the early stage. The question is: how does the catalyst/PE particle fragment/disintegrate if there is a certain amount of high molecular weight $\mathrm{PE}$ already present at the time when hydrogen is added?

In the second series, we performed the first step in the presence of a high hydrogen pressure (4 bar and 6 bar; 1 experiment was carried out (for comparison) at 0 bar hydrogen, (see Table 7.2). The system was depressurized after the first step and both the ethylene pressure and the hydrogen pressure were changed. In this way, the hydrogen: ethylene ratio was changed in the second step $(0,0.5$, and 2$)$. The experiment with 0 bar hydrogen during the first step was continued with a high hydrogen: ethylene ratio of 2 in the second step.

The question now becomes: How does the catalyst/PE particle fragment/disintegrate if - after a brittle (high crystalline, low $\mathrm{Mw}$ ) particle is formed - the growth stress and the molecular weight of the polymer produced change suddenly?

In the $3^{\text {rd }}$ series, the first gas-phase experiment was carried out by combining "low brittleness + high growth stress" (zero hydrogen +4 bar ethylene) in the first step with "high brittleness + moderate growth stress" ( 4 bar hydrogen +2 bar ethylene) in the second step.

The second experiment was exactly opposite to the first experiment: "high brittleness + moderate growth stress" ( 4 bar hydrogen +2 bar ethylene) in the first step combined with "low brittleness + high growth stress" (zero hydrogen +4 bar ethylene) in the second step.

For comparison with the results of the second experiment, a $3^{\text {rd }}$ experiment was carried out by combining "very high brittleness + moderate growth stress" ( 6 bar hydrogen +2 bar ethylene in the $1^{\text {st }}$ step) with "low brittleness + moderate growth stress" (zero hydrogen +2 bar ethylene) in the second step.

In the $4^{\text {th }}$ series, two experiments were performed. The first step of both experiments was performed with "high brittleness + moderate growth stress" (4 bar hydrogen +2 bar

\footnotetext{
${ }^{1}$ This is not completely true: during the hydrogen addition, the feed of ethylene is stopped due to the pressure set-point change of the mass flow controller, therefore, the partial pressure of ethylene decreases during the hydrogen feed by roughly 0.5 bar; however, after the $\mathrm{H}_{2}$ feed stops, hydrogen continues being absorbed by hexane - the gas phase pressure decreases by reaction + absorption, but is compensated by the ethylene feed only - this compensates for the ethylene loss...
} 
ethylene) in slurry. The second step was performed with "low brittleness + high and moderate growth stress respectively" (zero hydrogen +4 and 2 bar ethylene respectively) in the gasphase, after hexane evaporation.

In all the two-step experiments, Rp1 and Rp2 stand for the polymerization rates in the $1^{\text {st }}$ step and $2^{\text {nd }}$ steps respectively.

\subsection{Results}

\subsubsection{Slurry Polymerization: Hydrogen Feed in the $2^{\text {nd }}$ Step}

Table 7.1 summarizes the operating conditions and results of the first series. The results met the following expectations:

- the molecular weight decreases with increasing duration of the hydrogen impact from experiment 1 to experiment 5

- the crystallinity ("brittleness") follows the molecular weight trend and reaches the critical value near $75 \%$

- for the 1-step experiments 1 and 5, the polydispersity is low for experiment 1 (no hydrogen) and high for experiment 5 (presence of hydrogen) - same as was reported in Chapter 6; the maximum of polydispersity is reached for 20 minutes polymerization in the absence of $\mathrm{H}_{2}$ followed by 40 minutes polymerization in the presence of $\mathrm{H}_{2}$ - this value decreases with the increasing duration of step 2.

Table 7.1-Lists of operating conditions, 1-step without $\mathrm{H2}, \mathrm{PC} 2=2 \mathrm{bar}, \mathrm{T}=80^{\circ} \mathrm{C}$

\begin{tabular}{cccccccc}
\hline Run & $\begin{array}{c}\text { duration } \\
1^{\text {st }} / 2^{\text {nd }} \text { step (min) }\end{array}$ & $\begin{array}{c}1^{\text {st }}-2^{\text {nd }} \text { step } \\
\mathrm{P}_{\mathrm{H} 2}(\mathrm{bar})\end{array}$ & $\begin{array}{c}\mathrm{M}_{\mathrm{w}} \\
(\mathrm{kg} / \mathrm{mol})\end{array}$ & $\begin{array}{c}\mathrm{M}_{\mathrm{n}} \\
(\mathrm{kg} / \mathrm{mol})\end{array}$ & $\begin{array}{c}\mathrm{M}_{\mathrm{w}} / \mathrm{M}_{\mathrm{n}} \\
\mathrm{X}_{\mathrm{C} 1} \\
\%\end{array}$ & $\begin{array}{c}\mathrm{X}_{\mathrm{C} 2} \\
\%\end{array}$ \\
\hline 1 & $60 / 0$ & $0-0$ & 560 & 114 & 4.9 & 67 & 60.4 \\
2 & $20-40$ & $0-6$ & 335 & 8.3 & 40.5 & 70.1 & 73.7 \\
3 & $12-48$ & $0-6$ & 167 & 6.9 & 24.1 & 73.7 & 79.1 \\
4 & $6-54$ & $0-6$ & 105 & 6.8 & 15.5 & 73.7 & 80 \\
5 & $0-60$ & $0-6$ & 71.3 & 6.2 & 11.4 & 74.7 & 80.3 \\
\hline
\end{tabular}

A sudden increase in hydrogen pressure from 0 to 6 bar at 6,12 and 20 minutes reduced the reaction rate very slowly but after a few minutes it increased and reached the same level of reaction rate as in the absence of hydrogen; see Figure 7.1.

This is contrary to our findings so far, because:

- based on the result reported in Chapter 6 , the reaction rate in slurry at $80^{\circ} \mathrm{C}$ increased by increasing hydrogen pressure. 
- the ethylene pressure is higher for the second step, as proved by the sorption experiments.

How can this be explained? In Chapter 6, the hydrogen effect on the polymerization rate was addressed by two counter current effects: the hydrogen retarding effect (chemical) and the hydrogen enhancement effect (polymer mobility). The balance between these two effects explains the increasing or decreasing polymerization rate. We observed an increasing reaction rate in the presence of hydrogen in slurry due to the logical chain:

- high hydrogen pressure causes low molecular weight

- low molecular weight leads to high crystallinity

- high crystallinity causes high brittleness

- highly brittle particles fragment faster (or disintegrate)

- faster fragmentation causes new active site generation

- more active sites increase the polymerization rate (after activation with cocatalyst).

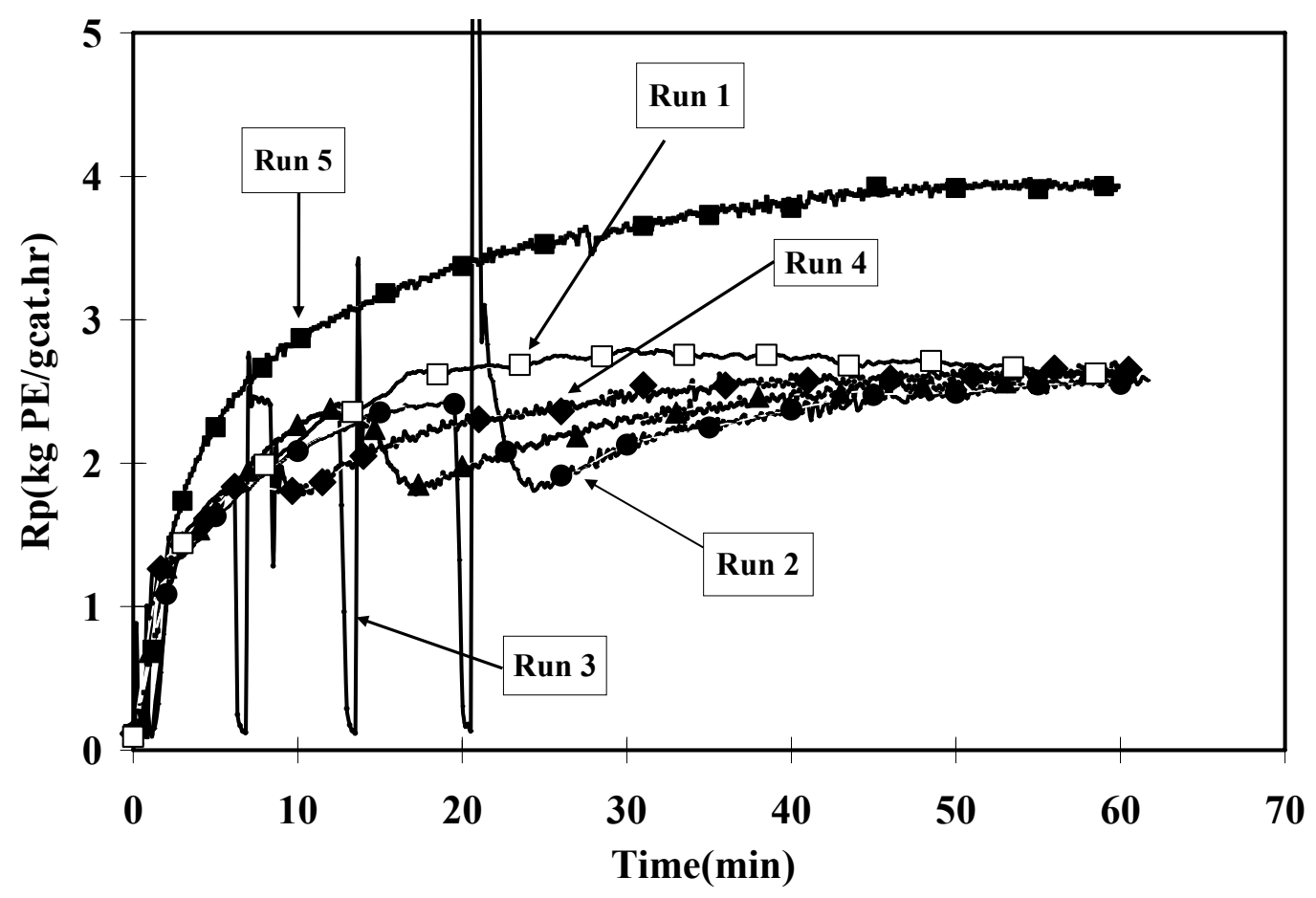

Figure 7.1-Hydrogen influence on two steps slurry polymerization for $\mathrm{Cg}$ catalyst at $\mathrm{PC} 2=\mathbf{2 b a r}$ and $\mathrm{T}=\mathbf{8 0}{ }^{\circ} \mathrm{C}$

Based on these observations, we can explain Figure 7.1 as described below (see also the particle size distribution in Figure 7.3).

Adding 6 bar hydrogen at the beginning leads to a high polymerization rate by extreme disintegration of the particles as explained above (experiment 5), whereas experiment 1 (without hydrogen $=$ low brittleness) shows the minimum disintegration. After a small period 
of polymerization without hydrogen, 6 minutes in experiment 4 for example, the hydrogen addition leads to the expected results: the disintegration decreases dramatically - clearly, the particle is less brittle after producing some high-Mw polymer (ductile polymer). This fits the theory developed in this thesis so far.

However, why does the rate curve of all experiments with different $\mathrm{H}_{2}$ injection times approach the rate profile of experiment 1 ? If the $\mathrm{H}_{2}$ addition would cause only a chemical change in the activity of all sites, then this behaviour cannot be explained. If we take into account the fact that internal fragmentation also causes generation of new sites then that behaviour becomes explainable: adding $\mathrm{H}_{2}$ at different times causes first a lower polymerization rate due to the chemical effect on currently existing active sites - the number of active sites is smaller in experiments running first under less brittle conditions, therefore, the number of active sites in experiments 2,3,4 is smaller than in experiment 5 at the moment of $\mathrm{H}_{2}$ addition, because the fragmentation is not complete in these experiments. Afterwards, the internal fragmentation proceeds, setting new active sites free and the same level of activity is reached - it only takes longer and is not joined by external fragmentation (disintegration), but the fragmentation runs internally. The same number of active sites is finally generated in experiments 2, 3, 4 and 5-now with only less external fragmentation in 2, 3, and 4 due to the presence of non-brittle PE. This is excellent evidence to support our theory.

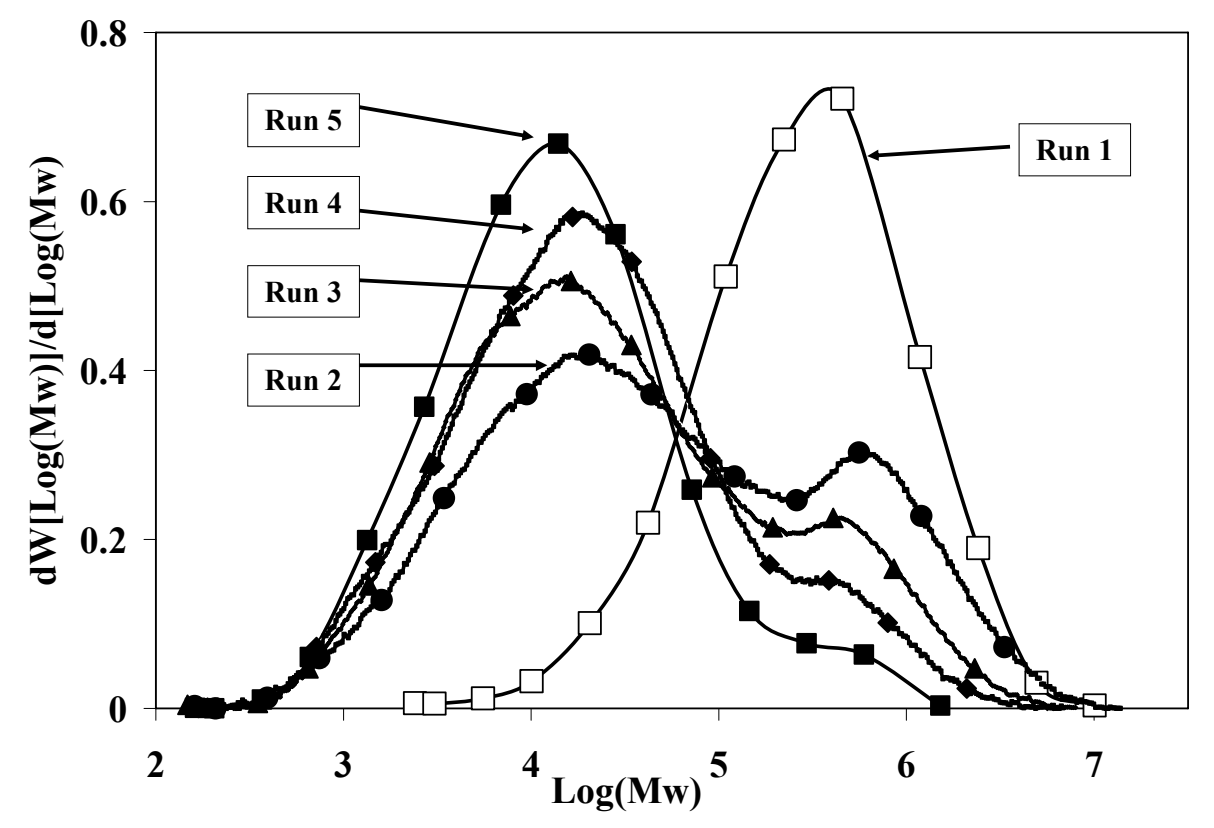

Figure 7.2-Hydrogen influence on MWD of two steps slurry polymerization for gas-phase catalyst, $\mathrm{C}_{\mathrm{g}}$, at $\mathrm{P}_{\mathrm{C} 2}=2 \mathrm{bar}$ and $\mathrm{T}=80^{\circ} \mathrm{C}$

Figure 7.2 reveals that MWD changes from unimodal distribution to bimodal distribution by introducing hydrogen in the second step. The height of shoulders at the low and high molecular weight regions depends on the yield of the produced polymer in each step. 
The higher the contribution of the low molecular weight part the more brittle is the polymer produced. This conforms absolutely with the particle disintegration; see Figure 7.3. One can interpret the first step of these two-step experiments as "prepolymerization" showing that pre-polymerization can be a good solution for decreasing fines generation for this particular catalyst in the slurry condition.

This is demonstrated impressingly with experiment 4: the naturally small difference between the MWD of experiment 4 (hydrogen addition after 6 minutes) and 5 (hydrogen addition at the beginning) leads to the largest reduction of the fines generation - this is a typical "prepolymerization effect" in terms of fines generation. Although reached the critical high crystallinity, the particles did not disintegrate too much if there is some ductile PE formed before $\mathrm{H}_{2}$ addition. It is not completely clear how much the ductile skin [82] formed around the growing particle contributes to this effect. We assume that the ductile skin keeps the fragments inside the growing catalyst/polymer particle without disintegration even if the $2^{\text {nd }}$ step polymer shows a very high crystallinity.

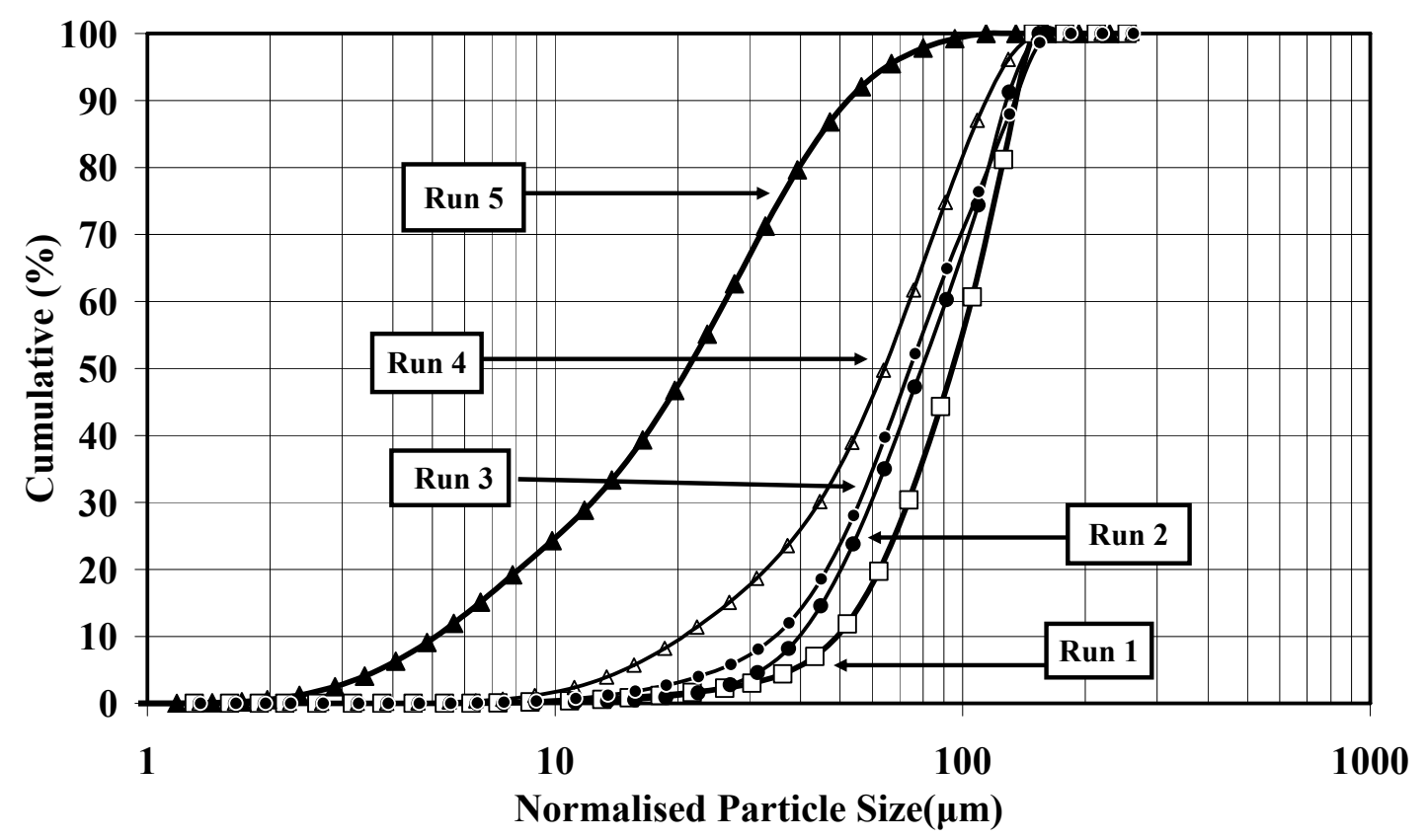

Figure 7.3-Hydrogen influence on cumulative PSD profiles normalized with the yield on slurry ethylene polymerization for $\mathrm{Cg}$ catalyst at $\mathrm{PC} 2=2$ bar $\mathrm{T}=80^{\circ} \mathrm{C}$

\subsubsection{Slurry Polymerization: Changing the Ethylene and the Hydrogen Pressure in the $2^{\text {nd }}$ Step}

A given amount of catalyst $(20 \mathrm{mg})$ was pre-contacted with TIBA for 30 minutes before being injected into the reactor filled with $700 \mathrm{ml}$-hexane, scavenged with $200 \mathrm{mg}$ TIBA and pressurized at a given gas composition and at constant $80^{\circ} \mathrm{C}$ as shown in Table 7.2. After performing the first step, a break of 10 to 30 minutes was required for cooling the reactor content, venting the $1^{\text {st }}$-stage gases, and for preparing the new gas composition for the second step. 
Table 7.2 -Operating conditions and yield ratio of two-step slurry experiments at $\mathrm{T}=\mathbf{8 0}^{\circ} \mathrm{C}$

\begin{tabular}{ccccc}
\hline Run & $\begin{array}{c}\text { duration } \\
1^{\text {st }} / 2^{\text {nd }} \text { step }(\min )\end{array}$ & $\begin{array}{c}\mathrm{P}_{\mathrm{C} 2}-\mathrm{P}_{\mathrm{H} 2} \\
1^{\text {st }} \text { step (bar) }\end{array}$ & $\begin{array}{c}\mathrm{P}_{\mathrm{C} 2}-\mathrm{P}_{\mathrm{H} 2} \\
2^{\text {nd }} \text { step (bar) }\end{array}$ & Yield ratio \\
\hline 1 & $29-66$ & $4-0$ & $2-4$ & 1.24 \\
2 & $61-32$ & $2-4$ & $4-2$ & 1.77 \\
3 & $51.5-14$ & $2-6$ & $4-0$ & 1.28 \\
4 & $29-16$ & $2-4$ & $2-0$ & 1.49 \\
\hline
\end{tabular}

* Yield ratio means the weight of polymer produced in the $2^{\text {nd }}$ step divided by that produced in the $1^{\text {st }}$ step

In terms of our theory, the 2-stage experimental plan can be characterized as shown in Table 7.3 .

Table 7.3-The stress types of produced PE in two-stage gas-phase polymerization

\begin{tabular}{lll}
\hline Run & \multicolumn{1}{c}{$\mathbf{1}^{\text {st }}$ Step } & \multicolumn{1}{c}{$\mathbf{2}^{\text {nd }}$ Step } \\
\hline 1 & Ductile PE + high growth stress & brittle PE + low growth stress \\
2 & Brittle PE + high growth stress & less brittle PE + high growth stress \\
3 & Brittle PE + high growth stress & ductile PE + high growth stress \\
4 & Brittle PE + high growth stress & ductile PE + low growth stress \\
\hline
\end{tabular}

Figure 7.4 shows the rate profiles. The profiles of the $1^{\text {st }}$ step of runs 2 and 4 (2bar $\mathrm{C}_{2}+4$ bar $\mathrm{H}_{2}$ ) and run 3 confirm the good reproducibility of our experiments. Comparison of $\mathrm{Rp} 2$ of these experiments reveals that removing hydrogen increases the reaction rate by means of the "chemical effect": the Rp2 decreases from experiment $3\left(4 \mathrm{bar} \mathrm{C}_{2}, \mathrm{no}_{2}\right)$ via experiment 2 ( 4 bar $\mathrm{C}_{2}, 2$ bar $\mathrm{H}_{2}$ ) to experiment 4 ( 2 bar $\mathrm{C}_{2}$, no $\mathrm{H}_{2}$ ). Of course, the latter is mainly influenced by the lower $\mathrm{C}_{2}$ pressure.

The comparison between experiments 3 and 1 is most interesting: Regarding the $\mathrm{C}_{2} / \mathrm{H}_{2}$ conditions, run 3 is a 'mirror image' of run 1 - only the run time differs a little and the hydrogen pressure is higher for first step of run 3 . With 4 bar $\mathrm{C}_{2}$ and zero $\mathrm{H}_{2}$, the $\mathrm{Rp} 2$ of run 3 is about twice as higher as the Rp1 of run 1. This difference can be explained to a large extent by the disintegration (new sites formation) within the first step of run 3 .

In experiment 3, the particle was highly fragmented, with some disintegration within step 1. Within 51 minutes, one can expect all potential active sites are converted to real active sites by reaching the maximum of fragmentation. In the second step, the two effects led to the high polymerization rate:

- the chemical effect (as often pointed out here: zero $\mathrm{H}_{2}$ leads to an increase in the polymerization rate)

- the fully fragmented catalyst contains the maximum possible number of active sites - the active site concentration in step 2 of run 3 is MUCH higher than the active site concentration in step 1 of run 1 . 
It is possible that this effect is strengthened by the activation effect: fragmentation is only one condition for producing active sites - the second condition is that sufficient co-catalyst must be available near the freshly produced active site. Even if the internal fragmentation proceeds in step 2 of experiment 1 , these fragments are covered by a huge amount of ductile PE - the co-catalyst can hardly reach the new sites at the internal surface against the polymer flow... and/or the concentration of the co-catalyst is too low near the new sites. Consequently, such sites cannot contribute to increasing the polymerization rate.

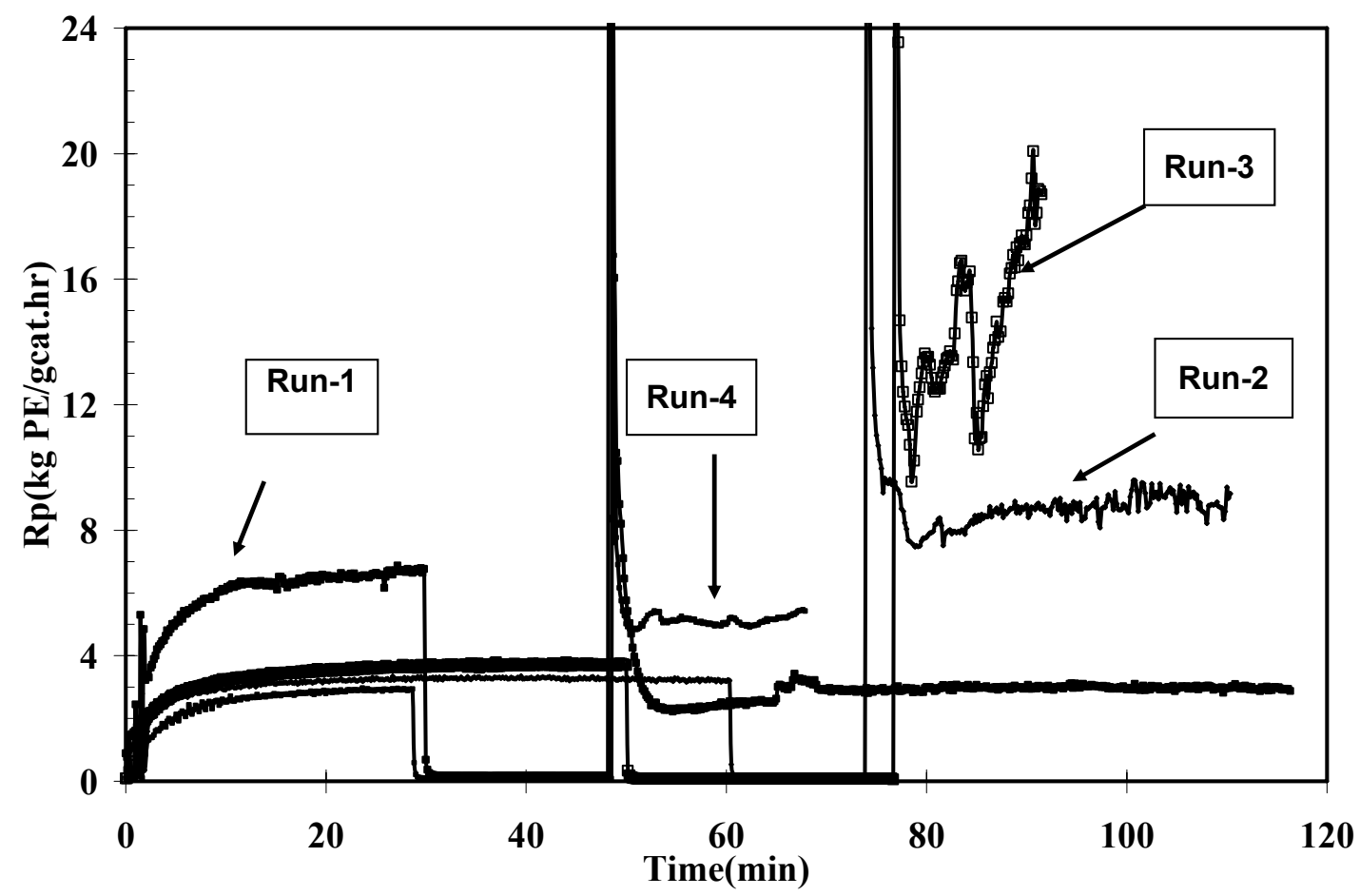

Figure 7.4-Effect of gas composition on the reaction rate-profile of two-steps slurry ethylene polymerization with Ziegler-Natta catalyst at $\mathrm{T}=\mathbf{8 0}^{\circ} \mathrm{C}$

$\mathrm{Rp} 2$ of run 2 is higher than $\mathrm{Rp} 1$ in run 1 despite the presence of the rate-decreasing hydrogen. This is clearly the consequence of the high degree of fragmentation in step 1 of run 2 : this effect cannot be explained by "mass transfer limitations".

Another interesting fact is that Rp2 of run 2 is lower than $\mathrm{Rp} 2$ of run 3: this is clearly the chemical effect of hydrogen and more particle fragmentation (more active site) of the first step of run 3. Figure 7.5 shows the highly porous surface of a sample produced under the same conditions as used for the $1^{\text {st }}$ step of run 3 .

Table 7.4 shows the results in terms of molecular weight and crystallinity. The results are as expected and are described below.

- $\quad$ The higher the $\mathrm{H}_{2}$ difference in the 2-stage process, the higher the polydispersity. Of course, this value depends on the amount of polymer produced in these two stages. The lowest polydispersity belongs to run 2 where both steps were performed in the presence of hydrogen. The highest polydispersity belonged to run 1, which produced 
the highest molecular weight in the first step without $\mathrm{H}_{2}$, while the $2^{\text {nd }}$ stage was carried out at the highest $\mathrm{H}_{2}: \mathrm{C}_{2}$ ratio of this experimental series.

- Both steps of run 2 were performed in the presence of hydrogen and provided the highest crystallinity and the lowest molecular weight - this is consistent with common experience.

- $\quad$ No matter which step was performed without hydrogen, whether the first or second step, the crystallinity was nearly identical - compare runs 1 and 4 .

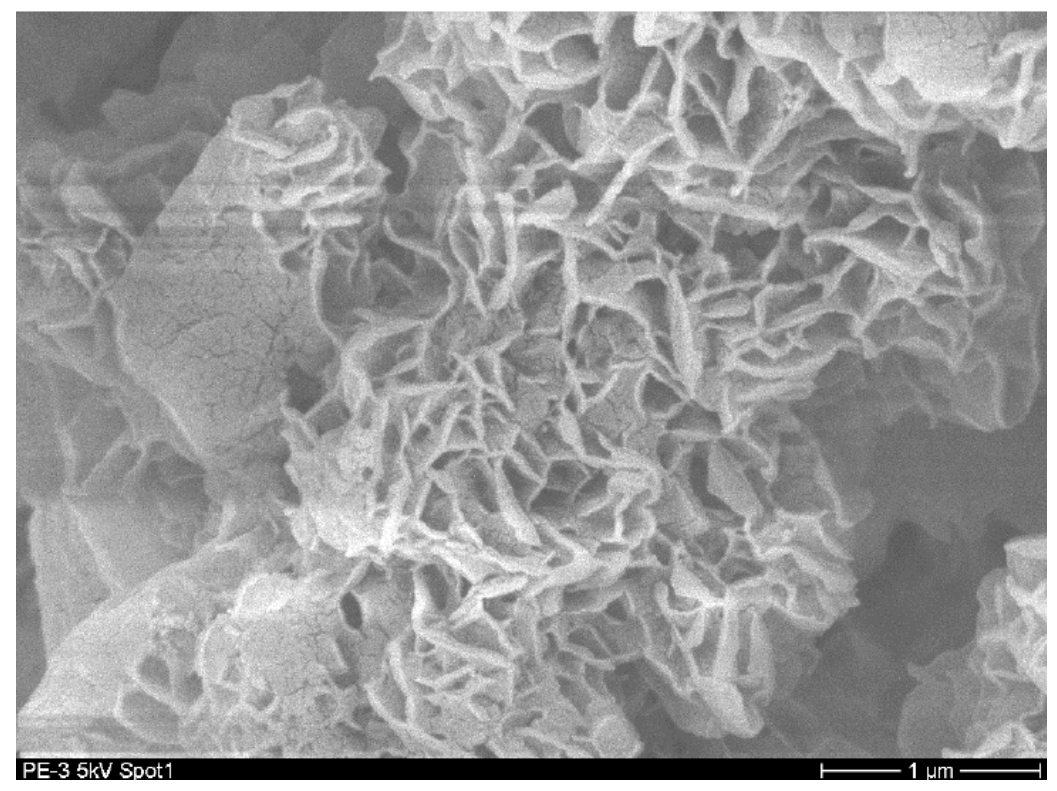

Figure 7.5-the SEM picture of polymer produced in slurry polymerization in presence of hydrogen at $\mathrm{P}_{\mathrm{C} 2}=\mathbf{2}$ bar and $\mathrm{T}=80^{\circ} \mathrm{C}$

It becomes clear that particles with the same brittleness (crystallinity) can show a very different polymerization rate profile in a two-stage process: the activity depends on which degree of fragmentation is reached in the first stage or - in other words - it depends on how many potential sites are converted to new sites by fragmentation that can be activated by the co-catalyst - if there is enough co-catalyst available near these new sites. We can surely assume that in the later stages, after producing a lot of ductile polymer, the activation of eventually freshly-produced new sites is a more difficult than after fragmentation during an earlier stage.

Table 7.4-Influence of gas composition on the properties of polymer produced in two-steps reaction

\begin{tabular}{cccccc}
\hline Run & $\begin{array}{c}\mathrm{M}_{\mathrm{w}} \\
(\mathrm{kg} / \mathrm{mol})\end{array}$ & $\begin{array}{c}\mathrm{M}_{\mathrm{n}} \\
(\mathrm{kg} / \mathrm{mol})\end{array}$ & $\mathrm{M}_{\mathrm{w}} / \mathrm{M}_{\mathrm{n}}$ & $\begin{array}{c}\mathrm{X}_{\mathrm{C} 1} \\
\%\end{array}$ & $\begin{array}{c}\mathrm{X}_{\mathrm{C} 2} \\
\%\end{array}$ \\
\hline 1 & 561.7 & 12.5 & 45 & 63.4 & 62.6 \\
2 & 128.7 & 10.3 & 12.5 & 80.3 & 88 \\
3 & 511.7 & 8.2 & 62.8 & 74.5 & 73.2 \\
4 & 361.9 & 18.4 & 19.7 & 65.5 & 66.7 \\
\hline
\end{tabular}


Figure 7.6 shows the MWD of the experiments. Explicit bi-modal MWD can be seen for runs 1, 3 and 4, that all perform one of steps with hydrogen and the other without hydrogen. The height of the low Mw and high Mw peak is a function of the polymerization rate reached during the relevant step. The MWD is a fingerprint of the polymerization rate of both steps. Both steps of run 2 were conducted with hydrogen - the MWD is relatively broad, but not bimodal.

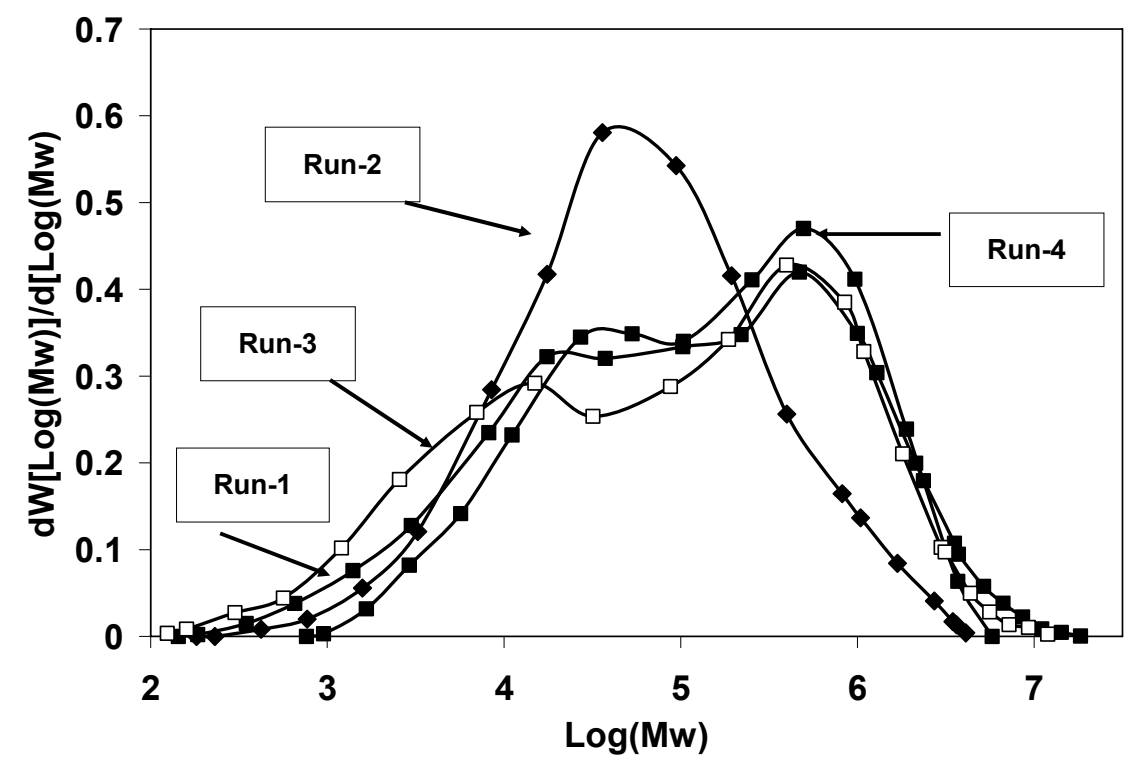

Figure 7.6-Operating conditions' influence on MWD of two-step slurry polymerization for Cg catalyst at $\mathbf{T}=\mathbf{8 0}{ }^{\circ} \mathrm{C}$

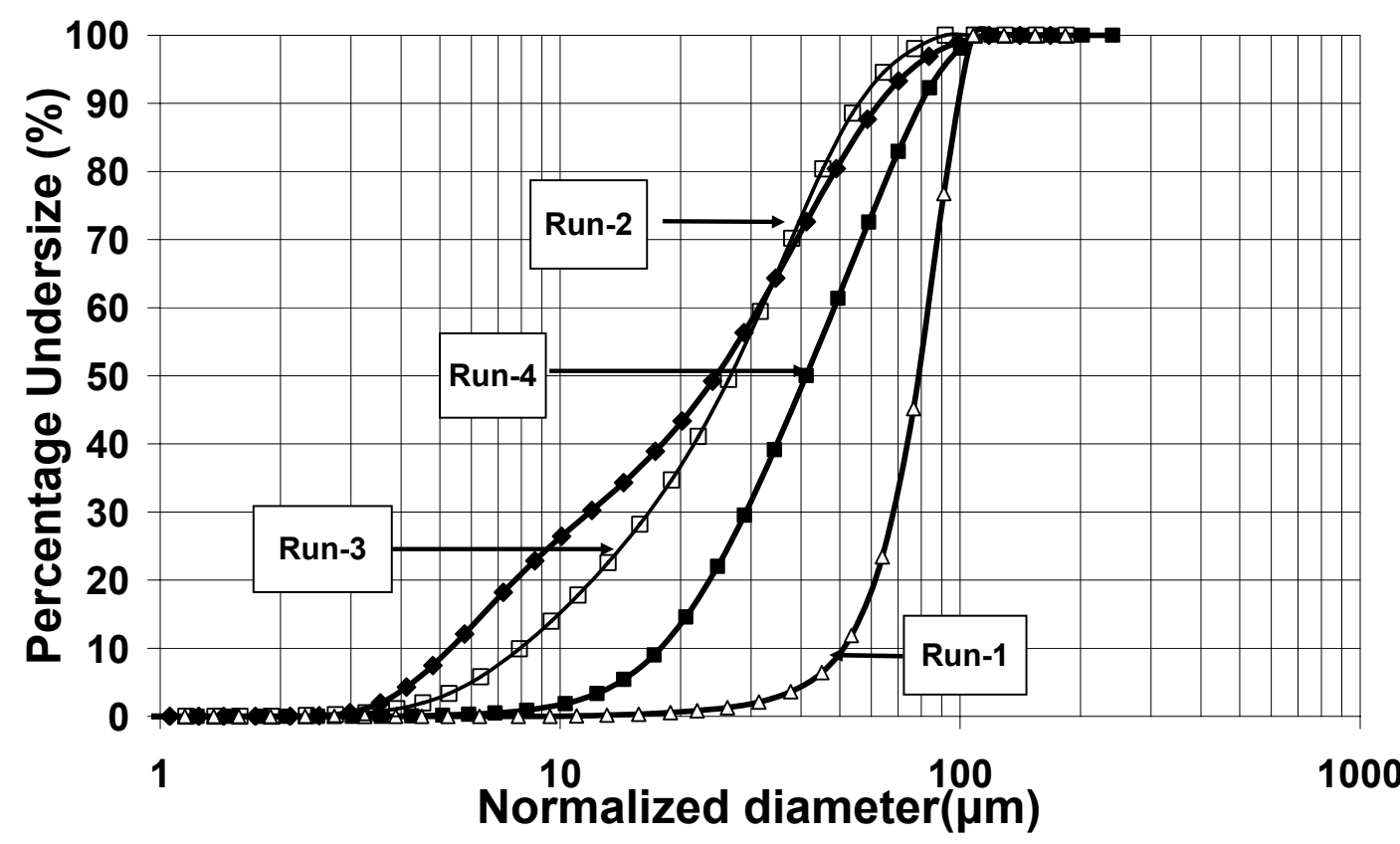

Figure 7.7-Operating conditions' influence on PSD normalized with the yield of two-step slurry polymerization for $\mathrm{Cg}$ catalyst at $\mathrm{T}=80^{\circ} \mathrm{C}$ 
Figure 7.7 shows the PSD profiles. The trends are explained below.

- $\quad$ As explained for the first series, forming ductile skin PE before hydrogen addition keeps the fragment particles inside the growing catalyst/particle. Therefore, it is not surprising that the PSD of run 1 is narrow and does not show fines formation.

- $\quad$ The existence of hydrogen in both steps (highest brittleness) of run 2 combined with a high growth stress in the $2^{\text {nd }}$ step leads to the highest fines generation.

- $\quad$ The high fines content of run 3 can be explained by the $1^{\text {st }}$ step which performed at highest hydrogen pressure (highest brittleness in $1^{\text {st }}$ step).

- $\quad$ The PSD profiles of run 4 is also clear - the $1^{\text {st }}$ step of run 4 was performed shorter than run 2 followed by the $2^{\text {nd }}$ step with less growth stress combined with less brittleness.

\subsubsection{Gas-Phase Polymerization: Changing the Ethylene and Hydrogen Pressure in the $2^{\text {nd }}$ Step}

Three two-step experiments were performed in the gas-phase. The same procedure as executed in the $2^{\text {nd }}$ series was followed for cooling and venting the gas contents from the first step, and making new gas composition for the $2^{\text {nd }}$ step. Table 7.5 summarizes the operating conditions and yield ratio (Y2/Y1) of the three experiments.

Table 7.5-Operating conditions and yield ratio of two-step gas-phase experiments at $T=80^{\circ} \mathrm{C}$

\begin{tabular}{ccccc}
\hline Run & $\begin{array}{c}\text { duration } \\
1^{\text {st }} / 2^{\text {nd }} \text { step }(\min )\end{array}$ & $\begin{array}{c}\mathrm{P}_{\mathrm{C} 2}-\mathrm{P}_{\mathrm{H} 2} \\
1^{\text {st }} \text { step (bar) }\end{array}$ & $\begin{array}{c}\mathrm{P}_{\mathrm{C} 2}-\mathrm{P}_{\mathrm{H} 2} \\
2^{\text {nd }} \text { step (bar) }\end{array}$ & Yield ratio \\
\hline 1 & $10.6-8.5$ & $4-0$ & $2-4$ & 0.2 \\
2 & $55.1-26.5$ & $2-4$ & $4-0$ & 0.45 \\
3 & $62-47$ & $2-6$ & $2-0$ & 0.84 \\
\hline
\end{tabular}

Table 7.6 shows the brittle/ductile behaviour of produced PE for each step, combined with the relevant growth stress produced by ethylene pressure. Based on the results reported in previous chapters ( 3,4 and especially 6$)$, the brittleness of produced PE in gasphase is lower than one produced in slurry under the same operating conditions.

Table 7.6-The stress types of produced PE in two-stage gas-phase polymerization

\begin{tabular}{lll}
\hline Run & \multicolumn{1}{c}{$\mathbf{1}^{\text {st }}$ Step } & \multicolumn{1}{c}{$\mathbf{2}^{\text {nd }}$ Step } \\
\hline 1 & Ductile PE + high growth stress & brittle PE + low growth stress \\
2 & Brittle PE + moderate growth stress & Ductile PE + high growth stress \\
3 & High Brittle PE + moderate growth stress & Ductile PE + moderate growth stress \\
\hline
\end{tabular}




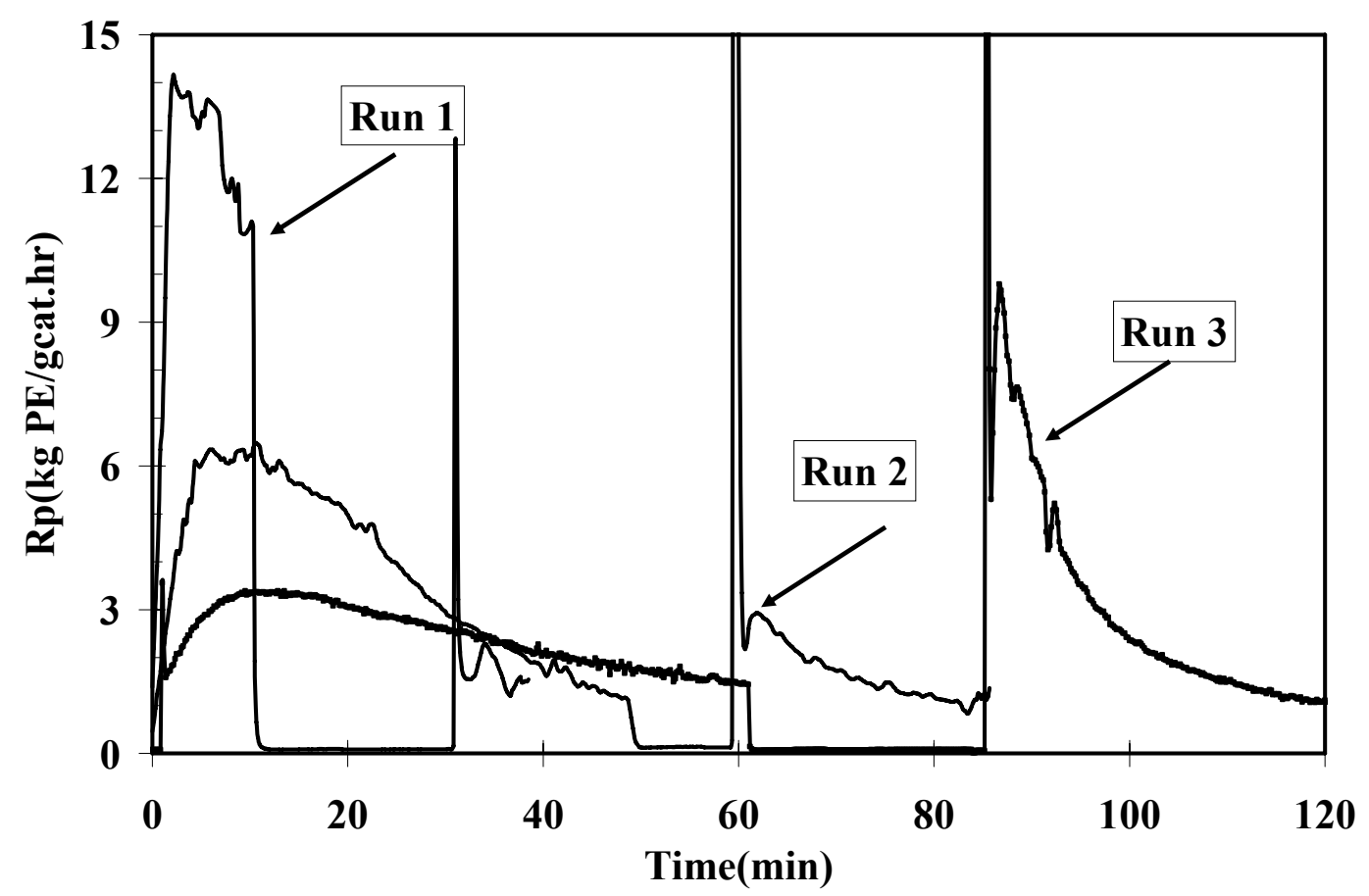

Figure 7.8-Effect of gas composition on the reaction rate-profile of two step gas-phase experiments at $\mathrm{T}=8^{\circ} \mathrm{C}$

The rate profiles are shown in Figure 7.8. Fast initiation of the $1^{\text {st }}$ step of run 1 ( 4 bar $\mathrm{C}_{2}$ and no hydrogen) was explained in Chapter 5 in terms of fast fragmentation accompanied by the fast generation of new active sites (auto-acceleration) due to a high ethylene pressure and particle overheating. Rp1 of run 2 and 3 is lower than to Rp1 of run 1, due to the lower ethylene pressure and the presence of hydrogen (chemical effect). Rp2 of run 1 is low, because the ethylene pressure is low and the hydrogen pressure is high compared to the $1^{\text {st }}$ run. $\mathrm{Rp} 2$ of run 2 is lower than to $\mathrm{Rp} 2$ of run 3 despite the lower ethylene pressure. The only possible explanation is the existence of more active sites due to higher fragmentation of the $1^{\text {st }}$ step of run 3 (higher hydrogen pressure combined with a longer reaction).

Table 7.7 shows molecular weight and crystallinity data for all three runs. All data is explainable based on what type (low/high molecular weight) PE and how much PE is produced in the $1^{\text {st }}$ step and the $2^{\text {nd }}$ step.

Table 7.7-Influence of gas composition on the properties of polymer produced in two-step gas phase experiments

\begin{tabular}{cccccc}
\hline Run & $\begin{array}{c}\mathrm{M}_{\mathrm{w}} \\
(\mathrm{kg} / \mathrm{mol})\end{array}$ & $\begin{array}{c}\mathrm{M}_{\mathrm{n}} \\
(\mathrm{kg} / \mathrm{mol})\end{array}$ & $\mathrm{M}_{\mathrm{w}} / \mathrm{M}_{\mathrm{n}}$ & $\begin{array}{c}\mathrm{X}_{\mathrm{C} 1} \\
\%\end{array}$ & $\begin{array}{c}\mathrm{X}_{\mathrm{C} 2} \\
\%\end{array}$ \\
\hline 1 & 600.5 & 83.1 & 7.2 & 57.1 & 60.7 \\
2 & 304.7 & 13.5 & 22.57 & 65.4 & 69.2 \\
3 & 537.2 & 9.4 & 57.2 & 76 & 77.3 \\
\hline
\end{tabular}


Figure 7.9 shows MWD of the three runs. Run 1 shows unimodal distribution with broad molecular weight due to a lower production of low molecular weight. The difference in the height of corresponding peaks of bimodal runs 2 and 3 represents the yield of the relevant step.

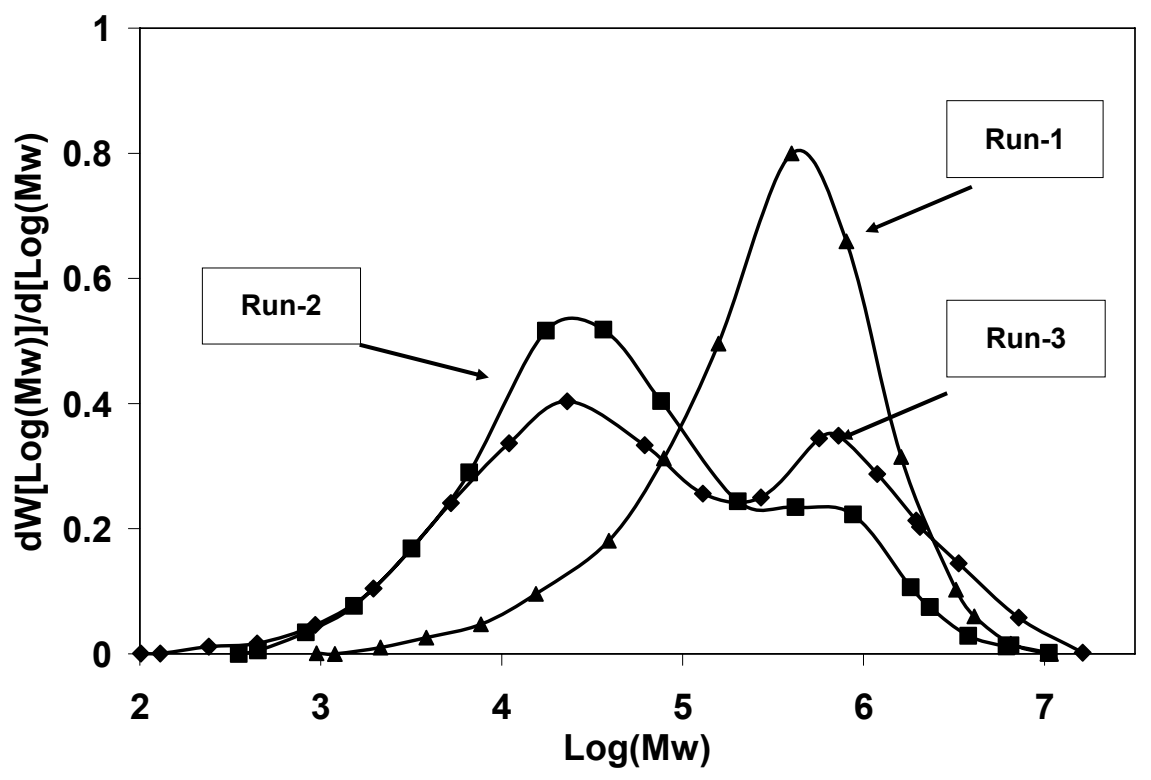

Figure 7.9-Operating conditions' influence on MWD of two-step gas-phase polymerization for $\mathrm{Cg}$ catalyst at $\mathbf{T}=\mathbf{8 0}{ }^{\circ} \mathrm{C}$

PSD profiles are shown in Figure 7.10. As expected, more fines were produced during run 3 due to the highly fragmented particles produced in the 1st step (high hydrogen pressure). Run 1 has the lowest fine content because the 1st step produce ductile PE.

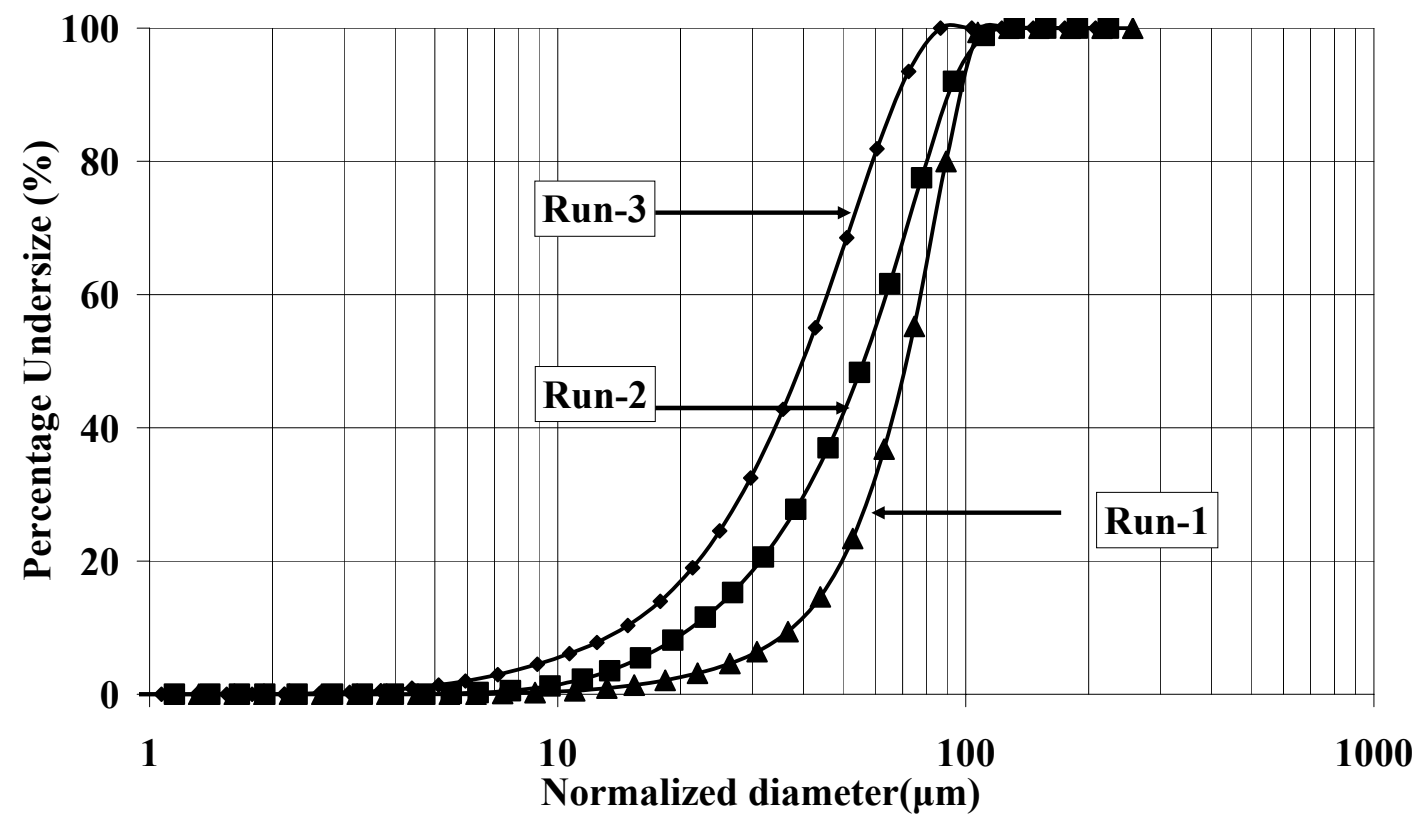

Figure 7.10-Operating conditions' influence on PSD normalized with the yield of two-step gasphase polymerization for $\mathrm{Cg}$ catalyst at $\mathrm{T}=80^{\circ} \mathrm{C}$ 


\subsubsection{Slurry and Gas-Phase Polymerization: Changing the Ethylene Pressure in the $2^{\text {nd }}$ Step}

Table 7.8 shows the operating conditions and yield ratio of the $4^{\text {th }}$ series of experiments. As can be seen, the ethylene and hydrogen pressures for all $1^{\text {st }}$ steps are the same. All phases for the $1^{\text {st }}$ step are slurry with the exception of run 4 which is gas-phase. All phases for the $2^{\text {nd }}$ step are gas-phase except run 3 which is slurry.

\begin{tabular}{|c|c|c|c|c|}
\hline Run & $\begin{array}{c}\text { duration } \\
1^{\text {st }} / 2^{\text {nd }} \text { step (min) }\end{array}$ & $\begin{array}{c}\mathrm{P}_{\mathrm{C} 2}-\mathrm{P}_{\mathrm{H} 2} \\
1^{\text {st }} \text { step (bar) }\end{array}$ & $\begin{array}{c}\mathrm{P}_{\mathrm{C} 2}-\mathrm{P}_{\mathrm{H} 2} \\
2^{\text {nd }} \text { step (bar) }\end{array}$ & Yield ratio ${ }^{*}$ \\
\hline 1 & $\begin{array}{c}46 / 42 \\
\text { slurry- gas-phase }\end{array}$ & $2-4$ & $4-0$ & 2.16 \\
\hline 2 & $\begin{array}{c}54 \text { / } 48 \\
\text { slurry - gas-phase }\end{array}$ & $2-4$ & $2-0$ & 1.5 \\
\hline 3 & $\begin{array}{c}29 \text { / } 16 \\
\text { slurry - slurry }\end{array}$ & $2-4$ & $2-0$ & 1.49 \\
\hline 4 & $\begin{array}{c}55 / 27 \\
\text { gas-phase - gas-phase }\end{array}$ & $2-4$ & $4-0$ & 0.45 \\
\hline
\end{tabular}

Table 7.9 shows the combinations of ductile/brittle behaviour of PE and growth stress for each step.

Table 7.9-The stress types of produced $P E$ in two-stage gas-phase polymerization

\begin{tabular}{lll}
\hline Run & \multicolumn{1}{c}{$\mathbf{1}^{\text {st }}$ Step } & \multicolumn{1}{c}{$\mathbf{2}^{\text {nd }}$ Step } \\
\hline 1 & Brittle PE + high growth stress & Ductile PE + high growth stress \\
2 & Brittle PE + high growth stress & Ductile PE + moderate growth stress \\
3 & Brittle PE + high growth stress & Ductile PE + moderate growth stress \\
4 & Brittle PE + high growth stress & Ductile PE + high growth stress \\
\hline
\end{tabular}

Figure 7.11 shows the rate profile for all runs. The rate profiles of the $1^{\text {st }}$ step of runs 1,2 and 3 confirm the reproducibility of experiments.

Comparison 1: runs 1 and 2

The only remarkable difference in the conditions is the higher $2^{\text {nd }}$ step growth stress (higher C2 pressure) in run 1. This is clearly reflected by the Rp2 of both experiments. As stated earlier, higher $\mathrm{Rp}$ in the gas-phase causes higher deactivation, and the "back-diffusion effect" and the "dilution effect" of the co-catalyst can contribute to this higher deactivation rate. The rate of both experiments is not affected by particle disintegration; therefore, the PSD is the same as shown in Figure 7.13. 
The crystallinity of run 1 is a little lower due to the higher molecular weight (see Table 7.10 and Figure 7.12) which is explained by the influence of the higher $\mathrm{C}_{2}$ pressure (see Chapter 5). The high polydispersity obtained in runs 1 and 2 can be attributed to the high yield of both steps.

The different initial increase of Rp2 in runs 1 and 2 can be attributed to various particles overheating. However, we cannot exclude the effect of the changing morphology by drying (i.e. removing hexane) between steps 1 and 2 - which can differ between runs 1 and 2 .

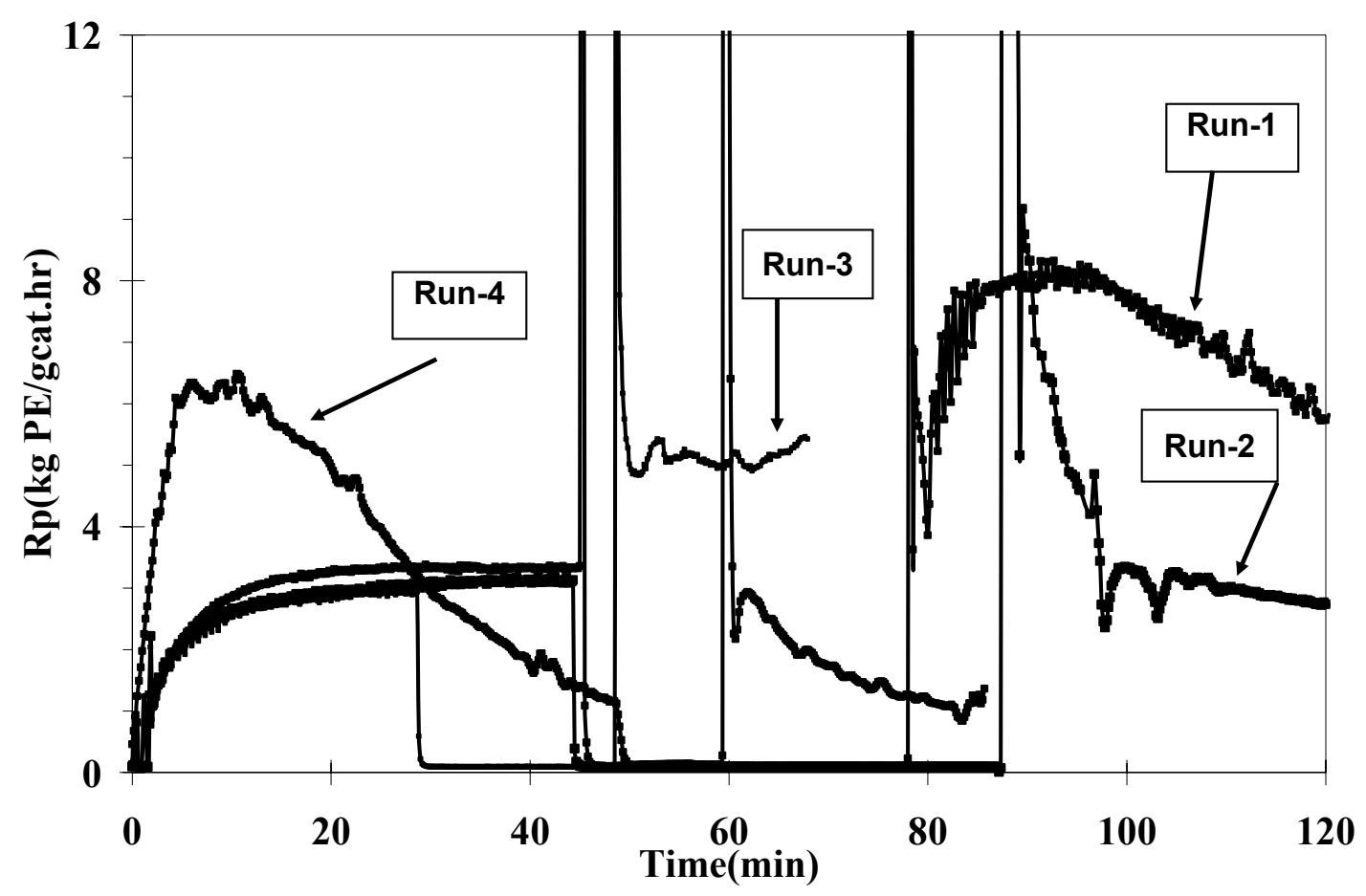

Figure 7.11 -Effect of gas composition on the reaction rate-profile of two-step experiments of $4^{\text {th }}$ series at $\mathbf{T}=80^{\circ} \mathrm{C}$

Comparison 2: run 2 and 3

These experiments differ by following the $2^{\text {nd }}$ step in different phases (slurry in run 3 , and gas-phase in run 2) and the time spent in both steps of each experiment are different. Although run 3 is faster, the ratio of polymer produced in both steps is nearly the same (see Table 7.8).

First, we comment on run 3: Rp2 is higher than Rp1, which is the well-known effect of hydrogen removal, as described in section 7.2.2. Furthermore, Rp2 in run 3 is almost constant, which is - incidentally - a good sign of not losing activity during the change of conditions between steps 1 and 2. Note that hexane was NOT removed between steps 1 and 2; therefore, one can expect that the in-situ polymer matrix will not change between these two steps. 
In run 2, step 2, the polymerization rate decreases dramatically, in huge contrast to run 3 . The reasons for such a difference between the gas-phase and slurry might include:

- Overheating in the gas-phase: usually it is assumed that there is no overheating after some pre-polymerization - the particle diameter is larger and the polymerization heat can be removed more easily. However, this cannot be true: if during the $1^{\text {st }}$ step ("pre-polymerization") more active sites are produced due to a high degree of fragmentation, then particle overheating can happen even after a relatively long-lasting $1^{\text {st }}$ step.

- $\quad$ Back-diffusion and dilution effect, see above

- Morphological collapse of the polymer matrix during the hexane removal accompanied by the kinetics of re-absorption of monomer in the $2^{\text {nd }}$ step. This effect of changing matrix properties can certainly affect the fragmentation and disintegration behaviour - for example, the dried polymer can accumulate a higher growth stress, and activation of new sites cannot be excluded even after a longlasting pre-polymerization (i.e. $1^{\text {st }}$ step), see Figure 7.5

Table 7.10-Influence of gas composition on the properties of polymer produced in two-step

\begin{tabular}{cccccc}
\multicolumn{6}{c}{ experiments of 4 ${ }^{\text {th }}$ series } \\
Run & $\begin{array}{c}\mathrm{M}_{\mathrm{w}} \\
(\mathrm{kg} / \mathrm{mol})\end{array}$ & $\begin{array}{c}\mathrm{M}_{\mathrm{n}} \\
(\mathrm{kg} / \mathrm{mol})\end{array}$ & $\mathrm{M}_{\mathrm{w}} / \mathrm{M}_{\mathrm{n}}$ & $\begin{array}{c}\mathrm{X}_{\mathrm{C} 1} \\
\%\end{array}$ & $\begin{array}{c}\mathrm{X}_{\mathrm{C} 2} \\
\%\end{array}$ \\
\hline 1 & 792.4 & 15.5 & 51.2 & 69.9 & 65.8 \\
2 & 824.3 & 12.6 & 65.4 & 74.9 & 73.6 \\
3 & 361.9 & 18.4 & 19.7 & 65.5 & 66.7 \\
4 & 304.7 & 13.5 & 22.57 & 65.4 & 69.2 \\
\hline
\end{tabular}

$M_{w}$ of run 3 is lower, for two reasons: first; the $2^{\text {nd }}$ step of run 3 performed in slurry and we know from previous chapters that polymers produced in slurry have lower $\mathrm{M}_{\mathrm{w}}$ compared to those produced in the gas-phase; second, the running times for both steps of run 3 are shorter and lead to lower $\mathrm{M}_{\mathrm{w}}$, as explained in Chapter 3.

Comparing the molecular weight and crystallinity of products from runs 2 and 3, Table 7.10 and Figure 7.12 shows less crystallinity for run 2 even with higher weight average molecular weight. This contradiction can be explained by a higher production of high crystalline polymer in the $1^{\text {st }}$ step of run 1 and a lower number average molecular weight obtained; see $\mathrm{M}_{\mathrm{n}}$ in Table 7.10.

In terms of PSD, Figure 7.13 reveals that run 2 produced more fines compared to run 3 . This can be attributed to particle overheating in the $2^{\text {nd }}$ run of run 2 which has performed in the gas-phase. Evaporation of hexane between the two steps of run 2 - changing the polymer matrix - could also be reason for this difference. 


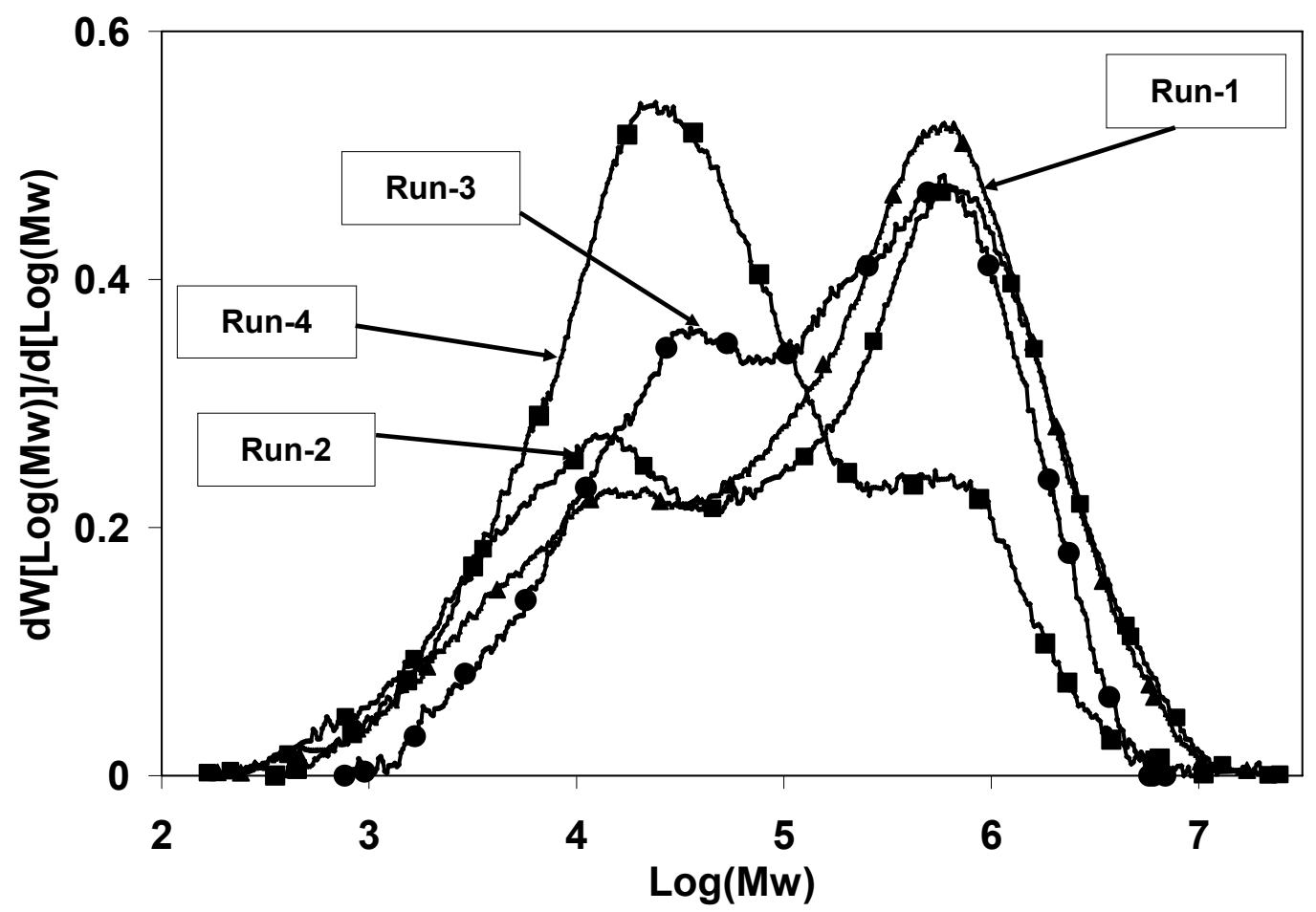

Figure 7.12-Operating conditions' influence on MWD of two-step experiments of 4th series at $\mathbf{T}=\mathbf{8 0}{ }^{\circ} \mathrm{C}$

Comparison 3: runs 1 and 4

The operating conditions are the same for the two runs. The main differences are that the $1^{\text {st }}$ step of run 4 is performed in the gas-phase and the yield ratio of run 4 is low. The rate profile of both runs was explained separately in section 7.2.3 for run 4 and in section 7.2.4 (comparison 1 and 2) for run 1. As can be seen in Figure 7.11, Rp2 of run 1 is very high compared to $\mathrm{Rp} 2$ in run 4, whereas the operating conditions of these gas-phase polymerization steps are similar.

The different conditions in step 1 of both experiments explain this difference as follows.

The co-catalyst back-mixing is limited in gas-phase polymerization and leads to a faster decay, therefore the high rate and the fast $1^{\text {st }}$ step decay of run 4 in the gas-phase is explainable. The high degree of co-catalyst back-mixing limitation leads to lower $\mathrm{Rp} 2$ for run 4. With the help of PSD (see figure 7.13) we can suggest another reason for the higher Rp2 in run 4: more active sites are available in the $1^{\text {st }}$ step of run 1 due to more disintegration in slurry.

The higher peak in the low molecular weight region for run 4 (see Figure 7.12) comes from a low yield ratio. The amount of high molecular weight produced in run 4 is low compared to that in run 1; see Table 7.10. 
Run 4 has the lowest fines production compared to all other runs due to performing the $1^{\text {st }}$ step in the gas-phase in which the influence of hydrogen on disintegration is lower compared to that to slurry.

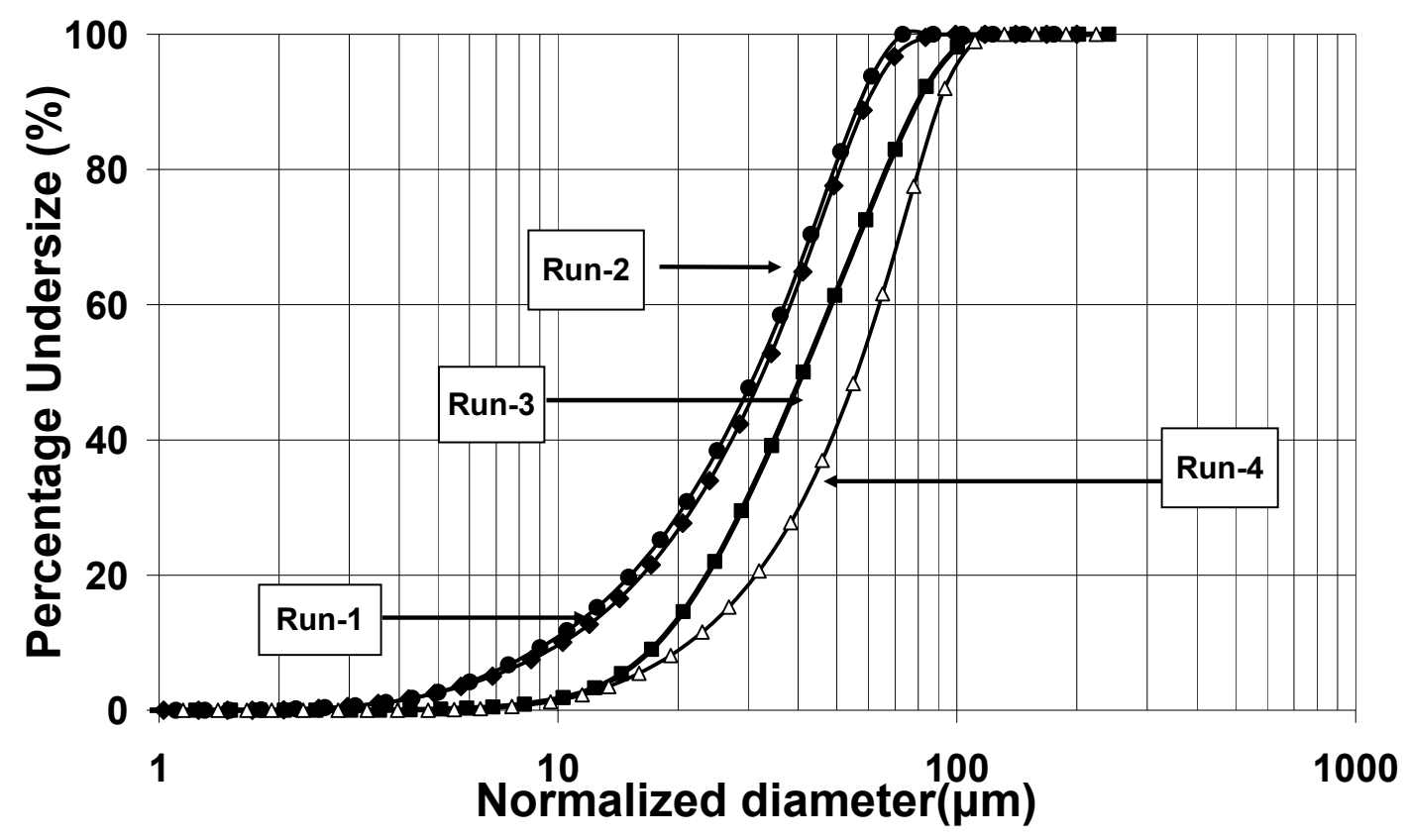

Figure 7.13-Operating conditions' influence on PSD normalized with the yield of two-step experiments of $4^{\text {th }}$ series at $T=80{ }^{\circ} \mathrm{C}$

\subsection{Conclusions}

Four series of two-stage experiments in different phases

- $\quad$ slurry-slurry (hydrogen feeds into $2^{\text {nd }}$ step)

- $\quad$ slurry-slurry (Change of ethylene and hydrogen Pressure in the $2^{\text {nd }}$ Step)

- gas-phase and slurry

- gas-phase and gas-phase

were carried out by varying the ethylene and hydrogen pressures to prove the GRAF hypothesis that has been developed in this thesis. Polymerization rate profiles, crystallinity, molecular weight and particle size were analyzed, leading to the following conclusions that strongly support this GRAF hypothesis.

- One can produce particles with identical crystallinity and MWD in 2-stage polymerizations, but the fragmentation behaviour of which can be absolutely different. The results reported in this chapter make clear that: the fragmentation behaviour and all related processes depend significantly on which kind of PE ductile or brittle - is produced in which step of the 2-stage polymerization. 
- The hydrogen enhancement effect in combination with disintegration of particles and new active site generation happens if hydrogen is only introduced at the beginning of the polymerization. Producing ductile polymer in the 1st step decreases the fragmentation-controlled enhancement effect of hydrogen. In onestage polymerizations (see Chapter 6), a crystallinity degree of $75 \%$ increased the brittleness such that disintegration of the particle results - although this is not strongly applicable in case of presence of ductile PE - experiment 4 of the $1^{\text {st }}$ series showed that a pre-polymerization of just $6 \mathrm{~min}$, producing ductile $\mathrm{PE}$ in the $1^{\text {st }}$ step, does not lead to disintegration of the particle, despite the extremely high crystallinity of about $80 \%$. No fines were generated in this experiment.

- The presence of ductile PE does not suppress particle fragmentation and the resulting rate enhancement completely, but the particle disintegration can be reduced.

- Removing hydrogen increases the reaction rate by the "chemical effect".

- The activity during the $2^{\text {nd }}$ step depends strongly on which degree of fragmentation is reached in the $1^{\text {st }}$ stage. However, for activation after fragmentation, the presence of the co-catalyst is required - "back-diffusion limitation" and the "dilution effect" can partially compensate for the fragmentation effect.

- Using the Cg catalyst, the lowest fines generation was found in a two-stage gasphase polymerization for bimodal PE production: the $1^{\text {st }}$ step without hydrogen (making ductile PE) and the $2^{\text {nd }}$ step with high hydrogen pressure (crystalline PE distributed within the ductile phase).

- Changing the polymer matrix properties during switching from $1^{\text {st }}$ to $2^{\text {nd }}$ step conditions (by means of cooling, pressurizing, depressurizing, hexane evaporation, re-pressurizing) can influence rate profiles and PSD, especially when performing the $1^{\text {st }}$ step in slurry in the presence of high hydrogen pressure and the $2^{\text {nd }}$ step in the gas-phase. Mass transfer effects cannot be excluded, especially not for large molecules with low diffusion coefficients.

- If the particle produced in the $1^{\text {st }}$ step shows a high activity due to new sites being activation by fragmentation, particle overheating should be taken into account even for the $2^{\text {nd }}$ step in the case of the gas-phase. This means that prepolymerization does not provide an absolute guarantee of avoiding of overheating.

As expected, bimodal MWD can be produced if one of the two steps is performed in the presence of hydrogen and the other in the absence of hydrogen. The MWD is a fingerprint of the polymerization rate of both steps: the amount of polymer produced in each step can be predicted from the MWD. 



\section{Chapter 8}

\section{Summary and Recommendations}

\subsection{Summary}

The investigation described in the present work was carried out to compare, identify and evaluate precisely the influence of:

- hydrogen pressure ( 0 to 10 bar)

- temperature $\left(40\right.$ to $\left.90^{\circ} \mathrm{C}\right)$

- ethylene pressure (1 to 12 bar)

- two-stage operation (gas-gas; slurry-gas, slurry-slurry)

- amount of hexane (going from gas phase to slurry)

- $\quad$ pre-contacting time catalyst - cocatalyst

on slurry and gas-phase ethylene homo-polymerization. Polymerization rate profiles, molecular weight distribution (MWD), particle size distribution (PSD) and crystallinity of the produced polymer were measured to quantify both the similarities and differences between these processes. Always using the same $\mathrm{MgCl} 2$ supported $\mathrm{TiCl} 4$ (Ziegler-Natta) catalyst, activated by TIBA, the (sometimes huge) differences in gas phase and hexane-slurry experimental results can only be attributed to the process conditions near the active sites. These near-to-active-sites conditions depend on the properties of the carrier (brittlenes and ductility, porosity, particle size, micro-viscosity, sorption capacity etc.) and depend on properties of the components used; there is an extreme difference between the TIBA diffusion in slurry and the gas-phase.

The question is: how to quantify the interaction between particle fragmentation (in extreme cases, disintegration and fines generation) and the polymerization rate? Which processes contribute to this very complex interaction and how can they be characterized?

An initial hypothesis was proposed in Chapter 1, termed GRAF (i.e. "Growth Rate Acceleration by Fragmentation") and that formed the working platform of this reseaerch. At the beginning of the work, it was well known that particle fragmentation is different in gas and slurry phase polymerizations leading to different properties of the final products and resulting in different reactor performance. The GRAF hypothesis, developed chapter by chapter in this work, can now be considered as a semi-quantitative theory, and can be used to explain some of the most significant differences between gas phase and slurry ethylene homopolymerization processes; see Figure 8.1. 


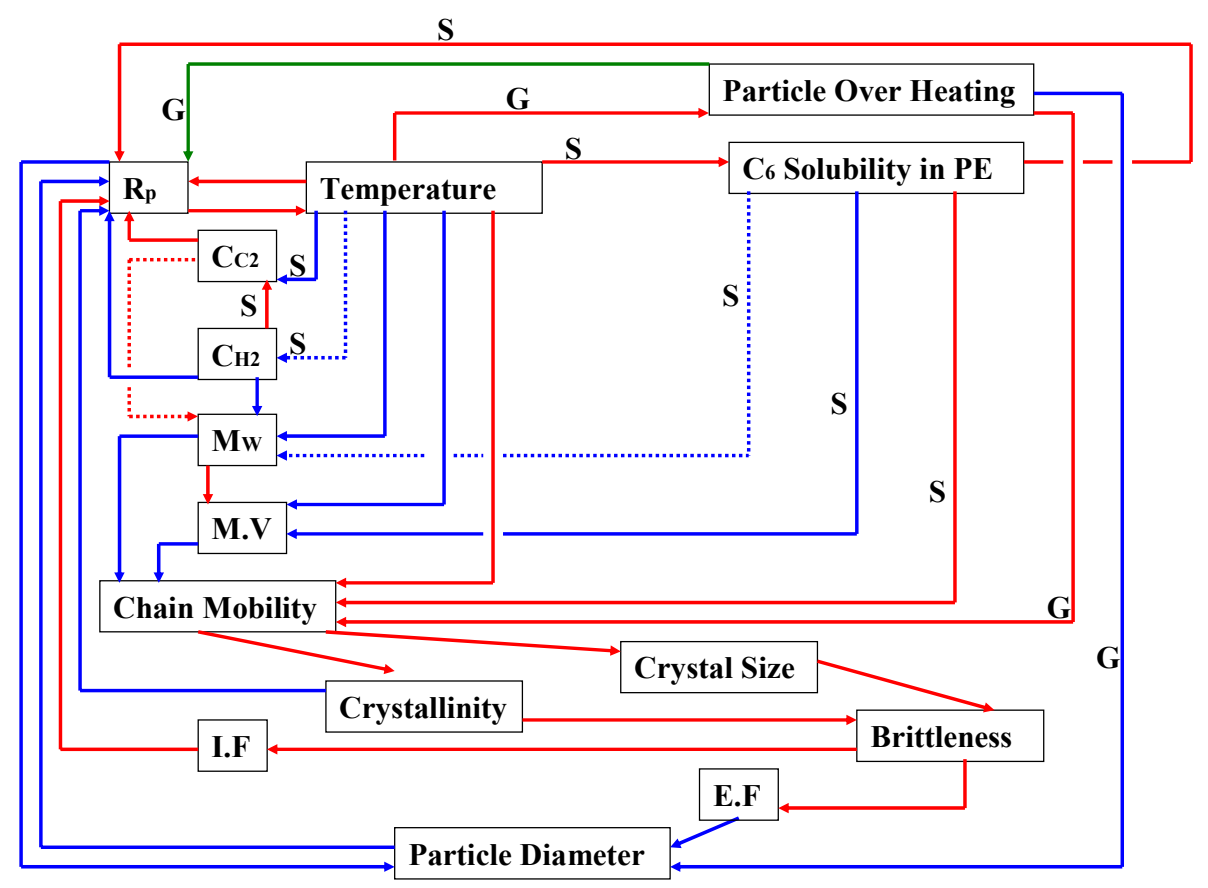

CC2 $=$ Ethylene Concentration,

CH2 $=$ Hydrogen Concentration,

C6=n-hexane

$\mathbf{R p}=$ Polymerization rate,

M.V= Micro viscosity,

$\mathbf{S}=$ Slurry,

G= Gas-phase

Mw= Molecular weight

$\mathbf{I} . \mathbf{F}=$ Internal Fragmentation

E.F= External Fragmentation

$A \longrightarrow B$ - It means B decreases with increasing $A$

$A \longrightarrow B$ - It means B increases with increasing $A$

$A \longrightarrow B$ - B may decreases or increases with increasing A

Figure 8.1- GRAF hypothesis of ethylene homo-polymerization using ZN catalysts

The influence of the hydrogen pressure $\left(\mathrm{P}_{\mathrm{H} 2}\right)$ serves as a good example of how to handle this scheme. Other examples are given in the chapter-by-chapter summary below:

- higher hydrogen pressure (higher $\mathrm{C}_{\mathrm{H} 2}$ ) leads to the well known chemical depression of the polymerization rate $\mathrm{Rp}$ (blue arrow between $\mathrm{C}_{\mathrm{H} 2}$ and $\mathrm{Rp}$, as shown in Figure 8.2: option 1).

Another effect of the higher $\mathrm{C}_{\mathrm{H} 2}$ is a decrease in the $\mathrm{Mw}$ of the polymer, as shown in Figure 8.2 , option 2, leading in turn to a lower micro-viscosity of the matrix. Additionally, smaller molecules show a higher chain mobility in a given matrix; therefore, the decreasing $\mathrm{Mw}$ 
increases the chain mobility double. This causes higher brittleness (again by a double effect) via higher crystallinity and the formation of larger crystals. Finally, higher brittleness under given growth stress causes faster and more intensive fragmentation (internal and/or external), which generates new sites -the activation of which increases the polymerization rate.

Option 2

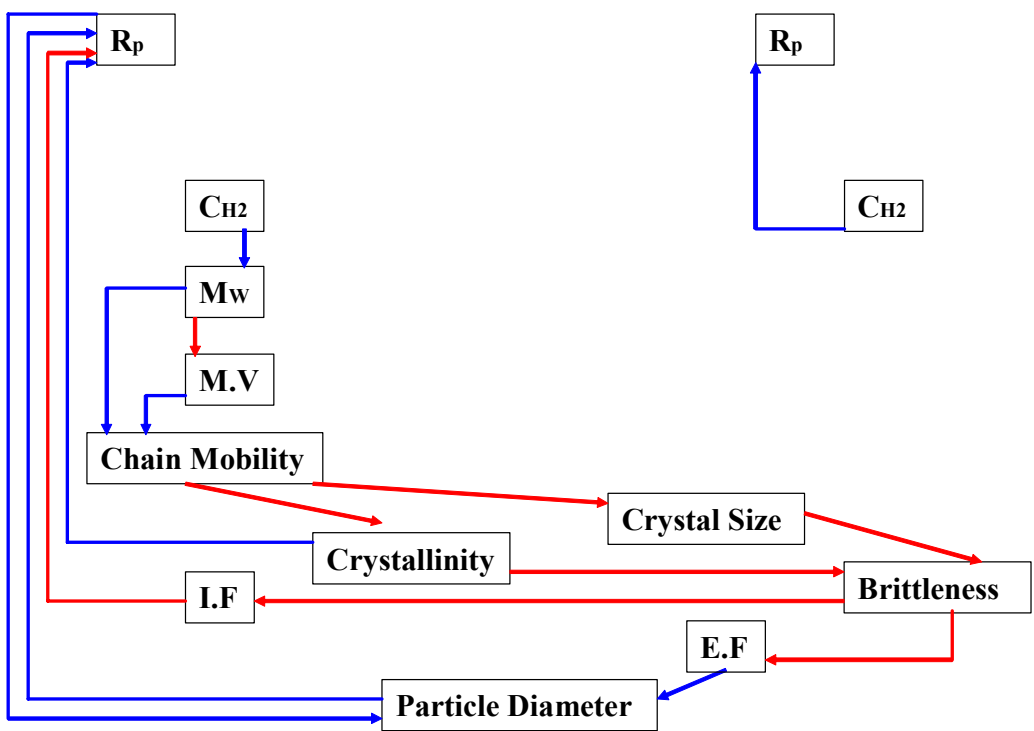

Figure 8.2-hydrogen influence on reaction rate

GRAF, represented in Figure 8.1, will be used in summarizing over all results found in this work.

\section{Chapter 1: Motivation}

By comparing slurry and gas-phase polyethylene processes both from the academic and industrial points of view, the complexity of processes around the active sites in slurry and the gas-phase during the ethylene homo-polymerization caused by different micro, meso and macro conditions is summarized, as previously published in the literature. These various conditions lead to different particle behaviour in gas and slurry, and especially cause different morphology and catalyst performance. However, it was not completely clear HOW, for example, the polymerization rate and morphology interact under the varying reaction conditions that exist in slurry and the gas-phase.

An initial hypothesis termed GRAF ("growth rate acceleration by fragmentation") was proposed, which was just an initial idea at the beginning of the work. We assumed that the GRAF hypothesis could probably be used to explain some of the differences between the gasphase and slurry ethylene homo-polymerization processes.

\section{Chapter 2: Experimental and theoretical methods}

Precise measurements of polymerization rate profiles, the characterization of the brittleness of the growing polymer particles, and the quantification of the disintegration of the particles are prerequisites for the GRAF evaluation can be found in Chapter 2. 
The 1.6-L stainless steel jacketed reactor, fully automated and operating at pressures up to 40 bar and temperatures up to $120^{\circ} \mathrm{C}$, was selected for both gas phase and slurry experiments. $\mathrm{n}$-hexane and salt were used as media for the slurry and gas-phase experiments, respectively. The reaction temperature and pressure were adjusted and controlled in the both isothermal (within $0.2{ }^{\circ} \mathrm{C}$ ) and isobaric (within 0.15 bar) modes. The same heterogeneous Ziegler-Natta (Z-N) catalyst system, which is used in gas-phase industrial ethylene polymerizations, was used in all experiments. The catalyst, with an average particle size of around $60 \mu \mathrm{m}$, consisted of a spherical $\mathrm{MgCl} 2-\mathrm{EtOH}$ support which was titanated with titanium chloride. Due to high sensitivity of the Z-N catalyst, all gases and liquids used were of 'polymer grade' and were purified in a series of purification columns. The instantaneous polymerization rate was measured by measuring the fed ethylene into the reactor via a mass flow meter to maintain a constant reactor pressure during the polymerization.

Among the various choices for characterizing polymer properties, we selected the most important polymer properties that we would expect to play a role in the GRAF hypothesis: molecular weight distribution (MWD), crystallinity and particle size distribution (PSD). Some samples were analyzed by means of transmission electron microscopy (TEM, for crystal size analysis) and scanning electron microscopy (SEM, for particle morphology).

It is widely accepted that heterogeneous Z-N catalysts are multi-site catalysts producing Flory-type MWD for each site. To estimate how different active sites respond to different reaction conditions, the MWD deconvolution technique was used. A five-site model can generally explain all MWDs of polymer produced in both slurry and gas-phase polymerizations.

Knowing the precise concentrations of ethylene and hydrogen and their interaction in the bulk of slurry and gas phase is an essential pre-condition for the interpretation of the reaction rate and of polymer properties such as MWD and crystallinity, in both slurry and the gas-phase. Aspen Polymer Plus software (version 11.1) was used to calculate the bulk concentrations of ethylene and hydrogen in slurry and the gas-phase.

\section{Chapter 3: Basic results}

Five series of experiments were conducted to validate the reaction conditions and reproducibility of experiments, and to answer the question: are the selected methods given in Chapter 2 optimal for developing the basic "GRAF" hypothesis that was defined in Chapter 1 ?

The following series were performed:

1. The reproducibility of the experiments was checked regarding the kinetics and polymer properties.

2. We moved systematically from the pure gas-phase to pure slurry conditions by increasing the solvent quantity from $2 \mathrm{ml}$ to $700 \mathrm{~mL}$.

3. The influence of the pre-contacting time on the reaction rate was investigated as were the produced polymer properties. 
4. The PSD was analyzed regarding replication phenomena; molecular weight and crystallinity were also investigated and compared.

5. The influence of inert gas (nitrogen) on the polymerization rate and polymer properties was investigated.

The results can be summarized as follows, with references to the five series described above:

For case 1: Good reproducibility of the experiments both in terms of kinetics and polymer properties was found. This implies a proper selection of reactor, the precision of all experimental methods used, excellent performance of all purification systems and precise control of operating variables such as temperature and pressure.

For case 2: Under the same operating conditions (ethylene and hydrogen pressure and temperature), gas-phase rate-profiles showed the "decay type curve" with rapid initiation followed by rapid decay, whereas slurry showed the "build up type curve" with formation of nearly-constant plateau activity after initiation. The fast initiation of the gas-phase profile is partially caused by local overheating of the growing particles and/or by different co-catalyst participation in the activation processes. The decay behaviour of the gas-phase experiments is explained by overheating during the initiation phase combined with a mass transfer limitation of co-catalyst ("back diffusion limitation") and co-catalyst dilution caused by the freshly produced polymer. Hexane is a good heat transfer medium and can contribute efficiently to the mass transfer of the co-catalyst. Therefore, in slurry local overheating is negligible and the homogeneity of co-catalyst near the active centres is high. In this sense, hexane is not at all "inert" - it affects all the relevant transport and equilibrium conditions. We showed that varying the amount of solvent could dramatically change the reaction rate profiles. Figure 8.1 shows schematically all processes explained above; see C6 solubility.

For case 3: Pre-contacting with co-catalyst for a certain time is essential for the $C_{g}$ catalyst. A short pre-contacting time leads to a low yield; in contrast, a long pre-contacting time results in fines formation. At least the effect of short pre-contacting times can be explained as in case 2: in terms of starved polymerization conditions regarding the co-catalyst. It remains unclear if the long pre-contacting time leads to too high a local stress at the beginning. Even catalyst destruction before polymerization cannot be excluded, and therefore a more thorough investigation is recommended.

For case 4: The longer the reaction time, the higher the molecular weight and the lower the crystallinity obtained. This is well known: the lower the molecular weight, the higher the crystallinity. The dependence on reaction time can be ascribed to the multi-site nature of $\mathrm{ZN}$ catalysts, since the low-Mw producing sites deactivate faster. (This result also shows the excellent purity of the monomer: in the semi-batch mode operation of the reactor, impurities would be enriched by the permanent feed and so would lead to lower Mw and faster deactivation.)

For case 5: The impact of partial pressure of nitrogen on the rate of reaction and the properties of the polymer produced is not significant. 


\section{Chapter 4: The influence of Temperature}

The temperature part of the GRAF theory, as shown in Figure 8.1, was developed by using the results of three series of experiments:

- $\quad 1^{\text {st }}$ series: slurry phase polymerization in the absence of hydrogen

- 2nd series: in slurry phase polymerization in the presence of hydrogen ( $\mathrm{PH} 2=2$ bar)

- $\quad 3^{\text {rd }}$ series: in gas phase polymerization in the presence of hydrogen $(\mathrm{PH} 2=2$ bar $)$

For $1^{\text {st }}$ series: the reaction rate increases with rising temperature, demonstrating the Arrhenius-type temperature dependency over the whole temperature range. For $2^{\text {nd }}$ and $3^{\text {rd }}$ series, this dependency was confirmed up to $80^{\circ} \mathrm{C}$ in both slurry and gas-phase. Further increase in temperature led to a $40 \%$ decrease in the polymerization rate. The faster deactivation (in the presence of hydrogen) at higher temperature $\left(90^{\circ} \mathrm{C}\right)$ can be explained by a faster deactivation during the first monomer addition after hydrogen transfer.

The overall activation energy of the polymerization rate for slurry ethylene polymerization in the presence of hydrogen is higher than that for the gas-phase.

The molecular weight of the polymer decreases with temperature and - of course - by the addition of hydrogen. The higher $\mathrm{Mw}$ at $90^{\circ} \mathrm{C}$ compared to $80^{\circ} \mathrm{C}$ (case A) can be explained by a lower deactivation rate for high-molecular weight producing sites than for to low molecular weight producing sites.

A clear increase in the high- Mw shoulder can been seen for all MWD of polymers produced in slurry in the presence of hydrogen as the temperature increases. This shoulder reveals that the Ziegler-Natta catalyst used in this study has various types of active centers showing various temperature dependencies. The active centers, which produce high molecular weight polymer, are less reactive to hydrogen at high temperature in slurry polymerization than in the gas-phase.

The polydispersity index (PD) significantly increased in the presence of hydrogen in both slurry and the gas-phase. Clearly, different active sites show different hydrogen responses, which leads to a broadening of the MWD.

In all three experimental series, raising the temperature increases the crystallinity of the polymers produced, which is in good agreement with the lowering of molecular weight. The increasing crystallinity is more pronounced in slurry ethylene polymerization in the presence of hydrogen. It demonstrates that the rate of the crystallization process is faster in slurry than in the gas-phase; see GRAF in Figure 8.1. Furthermore, in the absence of hydrogen, the first crystallinity is higher than the second (after re-crystallization). However, this order is reversed when hydrogen is present during the reaction.

The result of TEM shows that the larger lamellae (or the larger domain of small crystals) are produced at higher temperatures. This confirms that higher chain mobility leads to larger lamellae - which is a very important finding, because the larger the crystals near the critical crystallinity (found at about 75\%; "critical" in terms of disintegration), the easier the fragmentation proceeds, with fines production in the worst case.

In terms of particle size and particle size distribution, raising the temperature leads to different effects in three mentioned series. In the first series (slurry without hydrogen), no 
significant changes in PSD profiles were seen as the temperature increases. This shows that growth and thermal stresses due to rises in temperature do not break the growing catalystpolymer particle - the polymer is ductile enough not to disintegrate. When hydrogen was introduced in the second series, significant fines formation occurred at higher temperatures. We attributed this behaviour to the high crystallinity obtained. Whenever the measured crystallinity exceeded $70 \%$ for the $1^{\text {st }}$ run or exceeded $75 \%$ for the $2^{\text {nd }}$ run, fines were generated.

We conclude that for increasing temperature:

- The higher the temperature, the lower the molecular weight follows the well-known rule: transfer reactions increase more rapidly than propagation due to their higher activation energy

- In semi-batch slurry, the vapour pressure of hexane increases exponentially with temperature, causing a higher solubility of hexane in the amorphous polyethylene. This changes all equilibrium and transport properties within the particles, especially the solid phase viscosity and the diffusivity of all components (monomer, hydrogen, co-catalyst, and polymer).

- The higher mobility of freshly produced polymers within the polymer matrix, the viscosity of which is lowered, leads to faster crystallization. More and larger lamellae increase the brittleness of the particle. This promotes the fragmentation that can lead - in an extreme case - to shifting of the normalized PSD to the left and as a result fines are generated. Fragmentation, as a physical effect, generates new active sites, which in turn leads to a faster chemical reaction.

- In gas phase polymerizations, the overheating of particles must be taken into account. Especially at the beginning of the polymerization process, higher thermal and mechanical stress is caused while the polymerization rate is accelerating rapidly. Certainly, the fragmentation follows this course but does not lead to external fragmentation - because of the higher stickiness of the amorphous PE no fines are generated at higher temperatures. Fast deactivation during the later stage in gas phase can be caused by

- decreasing co-catalyst concentration around the active sites, the reason of which can be seen in the polymer flow from active sites to particle surface or in the dilution effect, see above

- thermal deactivation of some active sites during the overheating

Dilution and back-diffusion limitations of the co-catalyst are not present in slurry, because of the solubility of the co-catalyst in hexane accompanied by the viscosity-decreasing hexane solubility in the amorphous polyethylene.

\section{Chapter 5: Influence of Ethylene Pressure}

This chapter shows that changing ethylene pressure has a significant impact on GRAF. Three series of ethylene polymerizations under slurry and gas-phase conditions were performed. The 
influence of ethylene pressure on reaction rate-profiles, MWD, crystallinity and PSD was investigated as follows:

- varying the ethylene pressure in the absence of hydrogen in slurry

- varying the ethylene pressure under constant hydrogen pressure in gas phase

- varying the ethylene pressure under a constant hydrogen: ethylene ratio (gas and slurry)

In summary, the first order ethylene pressure dependency has been experimentally confirmed for all these series.

\section{For slurry polymerization in the absence of hydrogen:}

- The "build-up" profiles were obtained without any decay. A sufficiently good backdiffusion of co-catalyst is assumed to be the reason.

- Faster fragmentation accompanied by faster generation of new active sites (GRAF) at a higher ethylene pressure leads to a higher initial slope of the rate curves.

- As the ethylene pressure increases, the weight and number average molecular weight increases slightly (see Figure 8.1). This was explained in two ways; first, by the following equation as follows:

$$
\frac{1}{M_{n}} \approx \frac{k_{t M}}{k_{p}}+\frac{k_{t A}[A]}{k_{p}[M]}+\frac{k_{t S}}{k_{p}[M]}+\ldots \ldots
$$

Increasing monomer (ethylene) pressure decreases the second and third terms of the right-hand side of the equation, and consequently a higher molecular weight results at higher monomer pressures. Second, deconvolution of the MWD shows that - at higher ethylene pressures - the Flory molecular mass distribution contribution of low molecular weight producing centres decreases.

- A small shift of the PSD profile towards smaller particles was seen as the ethylene pressure increases. This can be attributed to a higher growth stress; see GRAF. The shift is more pronounced for bigger particles than for smaller particles. Clearly, big particles disintegrate more easily due to higher stress accumulation.

\section{For gas-phase polymerization at constant hydrogen pressure}

The following effects were found:

- "Decay" profiles were obtained. The higher the monomer pressure, the higher the peak activity and the faster the decay. This "chain of facts" can be explained in terms of GRAF as follows:

i. higher growth stress causes faster internal fragmentation, which leads to faster activation and higher peak activity, probably supported by some particle overheating

ii. a high polymerization rate causes a radial polymer flow away from active sites; this flow removes some co-catalyst from the active site ("co-catalyst back-diffusion limitation") and/or dilutes the co-catalyst 
concentration near the active sites ("dilution effect") - leading to lower activities.

- The slope of the decay rate curves shows a linear dependency on the ethylene pressure. Three reasons were discussed: (1) the overheating during the initial phase; (2) the "co-catalyst back diffusion effect"; and (3) the "dilution effect".

- The slope of the initiation rate curves shows an exponential ethylene pressure dependency. The reason for this is seen in an auto-acceleration mechanism involving "faster fragmentation accompanied by faster generation of new active sites", which is a key part of GRAF.

- Compared to slurry, gas-phase experiments show the higher initial slope of the initiation accompanied by a faster initiation time. This can be explained in terms of some rate-accelerating particles overheating.

- Despite the fact that the hydrogen: ethylene pressure ratio decreases as the ethylene pressure increases, no significant changes are observed in terms of MWD, $M_{w}$ and $\mathrm{M}_{\mathrm{n}}$. It was shown that increasing ethylene pressure might increase the solubility of hydrogen in the polymer structure leading to termination of more chains by hydrogen transfer.

- Despite the substantial change in growth stress as the ethylene pressure increases, the crystallinity did not reach the critical level, resulting no changing on the PSD profiles.

\section{For ethylene gas phase and slurry polymerization at constant hydrogen: ethylene ratio}

The following results were observed for increasing ethylene pressure at a constant $\mathrm{H}_{2}: \mathrm{C}_{2}$ ratio:

- faster activation followed by faster deactivation; the reason of both findings has already been explained, see above.

- $\quad$ significant increase in the average yield of the catalyst

- nearly constant crystallinity and melting temperature(Tm) as a consequence of the dominant hydrogen transfer

- large decrease in $\mathrm{Mw}$ and $\mathrm{Mn}$ accompanied by significant narrowing of the MWD; this behaviour can be explained by introducing a "solubility function" that explains why the hydrogen concentration increases with increasing ethylene pressure as shown in equation 5.12; the change in molecular weight of all three series described in this chapter can be explained by equation 5.12 .

- fines generation (particle disintegration) was negligible within the parameters varied.

\section{Chapter 6: Influence of Hydrogen Pressure}

The influences of hydrogen pressure on the polymerization kinetics and polymer microstructure characterization in gas-phase and slurry ethylene homo-polymerization were comprehensively investigated by performing two series of experiments, $1^{\text {st }}$ in slurry, $2^{\text {nd }}$ in the gas-phase. 
One of the most spectacular results was the counter effect of hydrogen. In the gasphase, the reaction rate decreases with increasing hydrogen pressure; but the opposite effect was found in the slurry phase. This different response can be explained in terms of GRAF; see above.

Hydrogen shows a similarly strong effect on the molecular weight of the polymer produced in either gas or slurry. In the absence of hydrogen, we found slightly lower molecular weights in slurry compared to the gas-phase. This can be explained by some more chain transfer in slurry, which can result directly from the hexane and/or from a higher contribution of the co-catalyst that reaches the active sites more easily in the presence of hexane (easier back diffusion). In the presence of the same hydrogen partial pressure in slurry and gas-phase, the MWDs of polyethylene produced polymerization differ only little. Whereas, the concentration ratio of hydrogen to ethylene in the bulk is about 13 times lower in slurry. Therefore, we have good reason to use the partial pressure in our modelling equations rather than the concentrations.

DSC results confirm that hydrogen addition increases the level of crystallinity coupled with a simultaneous decrease in the melting temperature. This correlates with the higher chain mobility of shorter chains.

Increasing the level of crystallinity can dramatically increase the production of fines in both phases and can change the particle size distribution accordingly if the brittleness of the crystalline particles and the growth stress reach critical levels.

In conclusion, the polymer mobility, which is influenced by many variables such as:

- temperature

- chain length of the polymer produced

- chain length of the dead polymer that surrounds the active sites ("matrix")

- hexane content in the amorphous part of the polymer matrix that changes the microviscosity

and these should be taken into account when interpreting the results. This different chain mobility leads to differences in the in-situ crystallinity, which has a direct impact on the particle brittleness. As a result, the particle can break at a critical growth stress that increases with the polymerization rate. This was the core result for the GRAF development. It is now very clear that this effect can affect the polymerization rate profiles in slurry and gas-phase polymerization differently due to different sorption, swelling and micro conditions around the active centres.

\section{Chapter 7: Two-stage Polymerization}

Four series of two-stage experiments in different phases:

- $\quad$ slurry-slurry (hydrogen feeds into the $2^{\text {nd }}$ step)

- $\quad$ slurry-slurry (change of ethylene and hydrogen pressure in the $2^{\text {nd }}$ Step)

- gas phase-slurry

- gas phase-gas phase

were carried out by varying the ethylene and hydrogen pressures to prove the GRAF hypothesis that has been developed in this thesis. According to the GRAF hypothesis, a quick 
change of polymerization conditions (in the $2^{\text {nd }}$ step) does not always lead to the same results, since the history of the particle (defined by the $1^{\text {st }}$ polymerization step) must determine the response. Again, polymerization rate profiles, crystallinity, molecular weight and particle size were analyzed, leading to the following results that strongly support the GRAF hypothesis.

- One can produce particles with identical crystallinity and MWD in 2-stage polymerizations, but the fragmentation behaviour can be markedly different. The results reported in this chapter make clear that the fragmentation behaviour and all related processes depend significantly on which kind of PE - ductile or brittle - is produced in which step of the two-stage polymerization.

- The hydrogen enhancement effect - in combination with the disintegration of particles leading to new active site generation - happens if hydrogen is introduced at the beginning of the polymerization. Producing ductile polymer in the 1st step decreases the fragmentation-controlled enhancement effect of hydrogen. In one-stage polymerizations (see Chapter 6), a crystallinity degree of $75 \%$ increased the brittleness such that intensive disintegration of the particle resulted. However, this is not the case in presence of sufficiently ductile PE fines generation can be suppressed in this case.

- In general, the presence of ductile PE does not suppress particle fragmentation and the resulting rate enhancement completely, but the particle disintegration can still be reduced dramatically. This is a useful tool for optimizing a catalyst.

- Removing hydrogen increases the reaction rate by the well-known "chemical effect", for which different explanations exists.

- The activity during the 2nd step depends strongly on what degree of fragmentation was reached in the 1 st stage. However, for activation of new sites after fragmentation, the presence of the co-catalyst is required - "back-diffusion limitation", and the "dilution effect" can partially compensate the rate accelerating fragmentation effect.

- The lowest fines generation was found in a two-stage gas phase polymerization for bimodal PE production: the 1st step without hydrogen (making ductile PE) and the 2nd step with high hydrogen pressure (crystalline PE distributed within the ductile phase).

- Changing the polymer matrix properties during switching from $1^{\text {st }}$ to $2^{\text {nd }}$ step conditions (by means of cooling, pressurizing, depressurizing, hexane evaporation, re-pressurizing) can influence both rate profiles and PSD. This is especially the case when performing the $1^{\text {st }}$ step in slurry under high hydrogen pressures and the $2^{\text {nd }}$ step in the gas-phase.

- If the particle produced in the $1^{\text {st }}$ step shows a high activity due to new sites generation by fragmentation, particle overheating should be taken into account even for the $2^{\text {nd }}$ step if it is carried out in the gas-phase. Therefore prepolymerization does not provide an absolute guarantee of avoiding overheating.

It is useful to analyze the MWD in terms of the GRAF hypothesis. The chain mobility plays an important role. Furthermore, the MWD is a fingerprint of the polymerization rate of both steps: the amount of polymer produced in each step can be predicted from the MWD. 


\subsection{Recommendations}

Extending the objectives of this thesis to ethylene co-polymerization with different copolymers under slurry and gas-phase conditions is extremely important from both the academic and industrial points of view. Therefore, we recommend performing a series of copolymerizations in slurry and gas-phase by varying

- hydrogen pressure,

- temperature

- ethylene pressure

- co-monomer types

- amount of co-monomer

- two-stage operation

- amount of hexane (going from gas phase to slurry).

Comparing ethylene homo/co-polymerization in the slurry and gas-phase in single-stage and two-stage processes using single-site catalyst is another recommendation. The interpretation of results is easier if one has to consider only one Flory component.

This thesis compared slurry and gas-phase ethylene homo-polymerization using an industrially applied gas- phase catalyst system. It is recommended to perform the same series of experiments using a typical slurry catalyst system.

The basic methods developed and used in this thesis - combined with the GRAF hypothesis form a powerful tool for developing more predictive models for studying the process technology of polyolefins. 


\section{Notation}

\begin{tabular}{|c|c|}
\hline$C^{*}$ & Number of active centers in a reaction \\
\hline$C_{H}^{*}$ & Temporarily deactivated centers by hydrogen \\
\hline $\mathrm{C}_{\mathrm{H} 2}$ & Hydrogen concentration, $\mathrm{g} / \mathrm{L}$ \\
\hline $\mathrm{C}_{\mathrm{C} 2}$ & Ethylene concentration, $\mathrm{g} / \mathrm{L}$ \\
\hline $\mathrm{C}_{\mathrm{g}}$ & Gas-phase catalyst \\
\hline $\mathrm{C}_{\mathrm{s}}$ & Slurry catalyst \\
\hline $\mathrm{DSC}$ & Differential Scanning Calorimetry \\
\hline$d_{c a t}$ & Catalyst diameter, $\mu m$ \\
\hline$d_{p o l}$ & Diameter of the polymer particle, $\mu m$ \\
\hline$E_{a, p}$ & Activation energy for the propagation \\
\hline$E$ & Ethylene \\
\hline EOS & Equations of state \\
\hline$f_{a}$ & Activation function \\
\hline GGE & Gas-phase ethylene polymerization using $\mathrm{C}_{\mathrm{g}}$ catalyst system \\
\hline GPC & Gel Permeation Chromatography \\
\hline GSE & Slurry ethylene polymerization using $C_{g}$ catalyst system \\
\hline HDPE & High density polyethylene \\
\hline$K_{H}$ & Henry's constant in bar.L /g \\
\hline$K_{p}$ & Modified propagation constant \\
\hline$K p_{0}$ & Pre-exponential factor \\
\hline$k$ & Boltzmann's constant, S-L EOS, J/K \\
\hline LDPE & Low density polyethylene \\
\hline LDPSA & Laser diffraction particle size Analyzer \\
\hline LLDPE & Linear low density polyethylene \\
\hline$M$ & Molecular weight S-L EOS \\
\hline $\mathrm{M}_{\mathrm{n}}$ & Number average molecular weight, $\mathrm{kg} / \mathrm{mol}$ \\
\hline$M_{T i}$ & Molar mass of Titanium \\
\hline $\mathrm{M}_{\mathrm{w}}$ & Weight average molecular weight, $\mathrm{kg} / \mathrm{mol}$ \\
\hline$m_{j}$ & Mass fraction of polymer produced by active site type $j$ \\
\hline$m_{i}$ & Weight fraction of component $i$, S-L EOS \\
\hline $\mathrm{MgCl}_{2}$ & Magnesium dichloride \\
\hline
\end{tabular}




\begin{tabular}{|c|c|}
\hline MWD & Molecular weight distribution \\
\hline$N^{*}$ & Number of active sites divided by Avogadro's number, mol \\
\hline$N_{T i}$ & The molar number of $\mathrm{Ti}$ atoms in the reactor \\
\hline $\mathrm{n}$ & Number of repeating unit \\
\hline$P$ & Actual pressure of the phase, S-L EOS, bar \\
\hline $\mathrm{P}$ & Pressure of the phase, bar \\
\hline$P^{*}$ & Characteristic pressure related to lattice variables S-L EOS, bar \\
\hline $\bar{P}$ & Reduced pressure of pure component, S-L EOS, dimensionless \\
\hline $\mathrm{P}_{\mathrm{H} 2}$ & Hydrogen partial pressure, bar \\
\hline$P_{C}$ & Critical pressure for pure component, S-L EOS, bar \\
\hline $\mathrm{P}_{\mathrm{C} 2}$ & Ethylene partial pressure, bar \\
\hline $\mathrm{P}_{\mathrm{N} 2}$ & Nitrogen partial pressure, bar \\
\hline $\mathrm{PE}$ & Polyethylene \\
\hline PSD & Particle size distribution \\
\hline PD & Polydispersity \\
\hline$R$ & Gas constant \\
\hline$R_{m}$ & Monomer consumption rate, $\mathrm{mol} / \mathrm{h}$ \\
\hline$R_{p}$ & Polymerization rate $\mathrm{kg} \mathrm{PE} / \mathrm{gcat} . \mathrm{hr}$ \\
\hline$R_{p a}$ & Average of $R_{p} \quad \mathrm{~kg} \mathrm{PE} /$ gcat.hr \\
\hline SEM & Scanning electron microscopy \\
\hline S-L EOS & Sanchez-Lacombe equation of state \\
\hline SRK & Soave-Redlich-Kwong \\
\hline$T$ & Reactor temperature, ${ }^{\circ} \mathrm{C}$ \\
\hline$T$ & Actual temperature of the phase, S-L EOS, ${ }^{\circ} \mathrm{C}$ \\
\hline$T^{*}$ & Characteristic temperature related to lattice variables, S-L EOS, ${ }^{\circ} \mathrm{C}$ \\
\hline$T_{C}$ & Critical temperature for pure component, SRK EOS, ${ }^{\circ} \mathrm{C}$ \\
\hline$T_{r}$ & Reduced temperature, SRK EOS, dimensionless \\
\hline $\bar{T}$ & Reduced temperature of pure component, S-L EOS, dimensionless \\
\hline $\mathrm{T}_{\mathrm{m}}$ & Melting temperature, ${ }^{\circ} \mathrm{C}$ \\
\hline TEA & Three ethyl aluminium \\
\hline TEM & Transmission electron microscopy \\
\hline TIBA & Triisobutyl aluminum \\
\hline$t_{\max }$ & Activation time \\
\hline UHMWPE & Ultra high molecular weight polyethylene \\
\hline
\end{tabular}




$\begin{array}{ll}\text { VLDPE } & \text { Very low-density polyethylene } \\ w_{r, j} & \begin{array}{l}\text { Instantaneous weight chain length distribution of the produced polymer on } \\ \text { active site } j \text { with the chain length of } r\end{array} \\ W_{r} & \text { Instantaneous weight chain length distribution of polymer } \\ \mathrm{X}_{\mathrm{C} 1} & \text { First crystallinity } \\ \mathrm{Xc}_{2} & \text { Second crystallinity } \\ \mathrm{X}_{\mathrm{C}(2-1)} & \text { Difference between } 1^{\text {st }} \text { and } 2^{\text {nd }} \text { crystallinity } \\ \mathrm{Y} & \text { Yield of polymer, } g_{\text {polymer }} / g \text {-cat } \\ \mathrm{Y} 1 & \text { Produced polyethylene after an hour of reaction } \mathrm{g} \\ \mathrm{Y} 2 & \text { Produced polyethylene after two hours of reaction } \mathrm{g} \\ y_{T i} & \text { Mass fraction of Titanium in the catalyst } \\ \mathrm{ZN} & \text { Ziegler-Natta catalyst }\end{array}$

\section{Greek letters}

$\begin{array}{ll}r & \text { Absolute density, } \mathrm{kg} / \mathrm{m} 3 \\ \eta_{i j} & \text { Binary interaction parameter, S-L EOS } \\ k_{i j} & \text { Binary interaction parameter, S-L EOS } \\ r^{*} & \text { Characteristic close-packed mass density, S-L EOS, } \mathrm{kg} / \mathrm{m3} \\ r_{m i x} & \text { mixture parameter, S-L EOS } \\ \emptyset_{i} & \text { volume of component } i, \mathrm{~S}-\mathrm{L} \text { EOS } \\ \varepsilon_{c a t} & \text { Porosity of catalyst, dimensionless } \\ \varepsilon_{p o l} & \text { Porosity of polymer, dimensionless } \\ \varepsilon^{*} & \text { Characteristic interaction energy per segment, S-L EOS, J/mol } \\ \varepsilon^{*}{ }_{i j} & \text { Cross parameter, S-L EOS } \\ \varepsilon^{*}{ }_{m i x} & \text { mixture parameter, S-L EOS } \\ \rho & \text { Actual density of pure component, S-L EOS, g/cm3 } \\ \rho_{c a t} & \text { Density of catalyst (support), } \mathrm{kg} / \mathrm{m}^{3} \\ \rho_{p o l} & \text { Density of polymer, } \mathrm{kg} / \mathrm{m}^{3} \\ \rho^{*} & \text { Characteristic density related to lattice variables, S-L EOS, } \mathrm{g} / \mathrm{cm} 3 \\ \bar{\rho} & \text { Reduced density of pure component, S-L EOS, dimensionless } \\ r & \text { Number of segments per chain, S-L EOS, } \\ r & \text { Chain length }\end{array}$


$\tau_{\mathrm{j}}$

$v^{*}$

$v^{*}{ }_{i j}$

$v_{\text {mix }}^{*}$

$\omega$

the ratio of the all rate of chain transfer to the rate of chain propagation on active site $j$ Closed-packed volume of a segment, S-L EOS,

Cross parameter, S-L EOS

Mixture parameter, S-L EOS

Acentric factor, SRK EOS 


\section{References}

1. Scheidl, K., Polyethylene-Polypropylene Chain Global PE/PP Industry Report. Maack Business Services, MaacK/Scheidl Partnership, Plastics Technology and Marketing 28thAnniversary 2008.

2. Frosch, R.A. and N.E. Gallopoulos, Strategies for Manufacturing. Scientific American, 1989. 261(3): p. 144-152.

3. Galli, P. and G. Vecellio, Technology: driving force behind innovation and growth of polyolefins. Progress in Polymer Science, 2001. 26(8): p. 12871336.

4. Galli, P. and G. Vecellio, Polyolefins: The most promising large-volume materials for the 21st century. Journal of Polymer Science Part a-Polymer Chemistry, 2004. 42(3): p. 396-415.

5. Tannous, K. and J.B.P. Soares, Gas-phase polymerization of ethylene using supported metallocene catalysts: Study of polymerization conditions. Macromolecular Chemistry and Physics, 2002. 203(13): p. 1895-1905.

6. Romano, U. and F. Garbassi, The environmental issue. A challenge for new generation polyolefins. Pure and Applied Chemistry, 2000. 72(7): p. 13831388.

7. Xie, T.Y., et al., Gas-Phase Ethylene Polymerization - Production Processes, Polymer Properties, and Reactor Modeling. Industrial \& Engineering Chemistry Research, 1994. 33(3): p. 449-479.

8. Andersson, L.H.U., B. Gustafsson, and T. Hjertberg, Crosslinking of bimodal polyethylene. Polymer, 2004. 45(8): p. 2577-2585.

9. Knuuttila, H., A. Lehtinen, and A. Nummila-Pakarinen, Advanced polyethylene technologies - Controlled material properties. Long-Term Properties of Polyolefins, 2004. 169: p. 13-27.

10. Montagna, A.A., R.M. Burkhart, and A.H. Dekmezian, The evolution of single-site catalysis. Chemtech, 1997. 27(12): p. 26-31.

11. Choi, K.Y. and W.H. Ray, Recent Developments in Transition-Metal Catalyzed Olefin Polymerization - a Survey .1. Ethylene Polymerization. Journal of Macromolecular Science-Reviews in Macromolecular Chemistry and Physics, 1985. C25(1): p. 1-55.

12. Severn, J.R., et al., "Bound but not gagged " - immobilizing single-site alphaolefin polymerization catalysts. Chemical Reviews, 2005. 105(11): p. 40734147.

13. www.lyondellbasell.com, Hostalen Process and Services.

14. Chemical Market resources, I., Status of Low Pressure PE Process Licensing. 2002. 7(6 ): p. 18 .

15. Jenkins, I.J.M., Jones; Russell L., Jones; Thomas M., Beret; Samil, "Method for fluidized bed polymerization" U.S. Patent 4588790, May 13, 1986

16. Samson, J.J.C., et al., Gas-phase polymerization of propylene with a highly active Ziegler-Natta catalyst. Aiche Journal, 1999. 45(7): p. 1548-1558.

17. Swogger, K.W., The impact of metallocene and constrained geometry catalyst technology on the plastics industry. Abstracts of Papers of the American Chemical Society, 1997. 213: p. 191-POLY. 
18. Kissin, Y.V., Homogeneous Interpretation of Ethylene Polymerization Kinetics with Supported Ziegler-Natta Catalysts. Journal of Molecular Catalysis, 1989. 56(1-3): p. 220-236.

19. Wonders, A.G., Moore, G. E., Ford, R. R., Daily, J. D., Dooley, K. A., and Garcia J.J., "Suppression of Fines in a Fluid Bed Polyethylene Process ", U.S. Patent 5969061, October 1999.

20. Gray, S.D., Coffy, T. J., Shamshoum, E. S., and Chen, H., "Polyolefin Catalysts, Production thereof and Method of Use", U.S. Patent 6846887, January, 2005.

21. Barbe, P.C., G. Cecchin, and L. Noristi, The Catalytic-System Ti-Complex Mgcl2. Advances in Polymer Science, 1986. 81: p. 1-81.

22. HanAdebekun, G.C., M. Hamba, and W.H. Ray, Kinetic study of gas phase olefin polymerization with a TiCl4MgCl2 catalyst.1. Effect of polymerization conditions. Journal of Polymer Science Part a-Polymer Chemistry, 1997. 35(10): p. 2063-2074.

23. Michaels, A.S. and H.J. Bixler, Solubility of Gases in Polyethylene. Journal of Polymer Science, 1961. 50(154): p. 393-\&.

24. Hutchinson, R.A. and W.H. Ray, Polymerization of Olefins through Heterogeneous Catalysis .8. Monomer Sorption Effects. Journal of Applied Polymer Science, 1990. 41(1-2): p. 51-81.

25. Weickert, G., et al., The particle as microreactor: catalytic propylene polymerizations with supported metallocenes and Ziegler-Natta catalysts. Chemical Engineering Science, 1999. 54(15-16): p. 3291-3296.

26. Pater, J.T.M., G. Weickert, and W.P.M. van Swaaij, Optical and infrared imaging of growing polyolefin particles. Aiche Journal, 2003. 49(2): p. 450464.

27. Di Martino, A., G. Weickert, and T.F.L. McKenna, Contributions to the experimental investigation of the nascent polymerisation of ethylene on supported catalysts, 1 - A quenched-flow apparatus for the study of particle morphology and nascent polymer properties. Macromolecular Reaction Engineering, 2007. 1(1): p. 165-184.

28. Ingram, P. and Schindle.A, Morphology of as-Polymerized Polyethylene .2. Electron Microscopy. Makromolekulare Chemie-Macromolecular Chemistry and Physics, 1968. 111(Feb): p. 267-\&.

29. Hutchinson, R.A., C.M. Chen, and W.H. Ray, Polymerization of Olefins through Heterogeneous Catalysis .10. Modeling of Particle Growth and Morphology. Journal of Applied Polymer Science, 1992. 44(8): p. 1389-1414.

30. Yiagopoulos, A., et al., Heat and mass transfer phenomena during the early growth of a catalyst particle in gas-phase olefin polymerization: the effect of prepolymerization temperature and time. Chemical Engineering Science, 2001. 56(13): p. 3979-3995.

31. Floyd, S., et al., Polymerization of Olefins through Heterogeneous Catalysis .4. Modeling of Heat and Mass-Transfer Resistance in the Polymer Particle Boundary-Layer. Journal of Applied Polymer Science, 1986. 31(7): p. 22312265.

32. Floyd, S., et al., Polymerization of Olefins through Heterogeneous Catalysis 6. Effect of Particle Heat and Mass-Transfer on Polymerization Behavior and Polymer Properties. Journal of Applied Polymer Science, 1987. 33(4): p. 1021-1065. 
33. Jejelowo, M.O., D.T. Lynch, and S.E. Wanke, Comparison of Ethylene Polymerization in Gas-Phase and Slurry Reactors. Macromolecules, 1991. 24(8): p. 1755-1761.

34. Wu, Q., H.H. Wang, and S.G. Lin, Gas-phase versus slurry copolymerization of ethylene with 1-butene over MgCl2-supported titanium catalysts after prepolymerization. Macromolecular Chemistry and Physics, 1996. 197(1): p. 155-163.

35. Marques, M.M.V., et al., Polymerization of Ethylene Using a High-Activity Ziegler-Natta Catalyst .1. Kinetic-Studies. Journal of Polymer Science Part aPolymer Chemistry, 1993. 31(1): p. 209-218.

36. Tait, P.J.T., A Kinetic Model for Hetrogeneeous Ziegler-Natta Polymerization, in Coordination Polymerization, J.C.W. Chien, Editor. 1975, Academic Press, Inc.: London. p. 155-197.

37. Kim, I., J.H. Kim, and S.I. Woo, Kinetic-Study of Ethylene Polymerization by Highly-Active Silica Supported Ticl4 Mgcl2 Catalysts. Journal of Applied Polymer Science, 1990. 39(4): p. 837-854.

38. HanAdebekun, G.C. and W.H. Ray, Polymerization of olefins through heterogeneous catalysis .17. Experimental study and model interpretation of some aspects of olefin polymerization over a TiCl4/MgCl2 catalyst. Journal of Applied Polymer Science, 1997. 65(6): p. 1037-1052.

39. Singh, D. and R.P. Merrill, Molecular Weight Distribution of Polyethylene Produced by Ziegler-Natta Catalysts. Macromolecules, 1971. 4(5): p. 599-\&.

40. Crabtree, J.R., et al., Role of Diffusion in Ziegler Polymerization of Ethylene. Journal of Applied Polymer Science, 1973. 17(3): p. 959-976.

41. Nagel, E.J., V.A. Kirillov, and W.H. Ray, Prediction of Molecular-Weight Distributions for High-Density Polyolefins. Industrial \& Engineering Chemistry Product Research and Development, 1980. 19(3): p. 372-379.

42. Debling, J.A. and W.H. Ray, Heat and Mass-Transfer Effects in Multistage Polymerization Processes - Impact Polypropylene. Industrial \& Engineering Chemistry Research, 1995. 34(10): p. 3466-3480.

43. Soares, J.B.P., Mathematical modelling of the microstructure of polyolefins made by coordination polymerization: a review. Chemical Engineering Science, 2001. 56(13): p. 4131-4153.

44. Dube, M.A., et al., Mathematical modeling of multicomponent chain-growth polymerizations in batch, semibatch, and continuous reactors: A review. Industrial \& Engineering Chemistry Research, 1997. 36(4): p. 966-1015.

45. Bergstra, M.F. and G. Weickert, Ethylene polymerization kinetics with a heterogeneous metallocene catalyst - Comparison of gas and slurry phases. Macromolecular Materials and Engineering, 2005. 290(6): p. 610-620.

46. Kissin, Y.V., et al., Kinetics and mechanism of ethylene homopolymerization and copolymerization reactions with heterogeneous Ti-based Ziegler-Natta catalysts. Topics in Catalysis, 1999. 7(1-4): p. 69-88.

47. Fernandes, F.A.N. and L.M.F. Lona, Fluidized bed reactor for polyethylene production. The influence of polyethylene prepolymerization. Brazilian Journal of Chemical Engineering, 2000. 17(2): p. 163-170.

48. Ushakova, T.M., et al., Peculiarities of homo- and copolymerization of ethylene with propylene using immobilized vanadium catalysts. Vysokomolekulyarnye Soedineniya Seriya a \& Seriya B, 1996. 38(2): p. 197202. 
49. Meier, G.B., G. Weickert, and W.P.M. van Swaaij, Comparison of gas- and liquid-phase polymerization of propylene with heterogeneous metallocene catalyst. Journal of Applied Polymer Science, 2001. 81(5): p. 1193-1206.

50. Banat, Y., "Fines Generation in Gas-Phase Ethylene Polymerization", Ph.D Thesis. 2006, University of Twente.

51. Zucchini, U. and G. Cecchin, Control of Molecular-Weight Distribution in Polyolefins Synthesized with Ziegler-Natta Catalytic-Systems. Advances in Polymer Science, 1983. 51: p. 101-153.

52. Floyd, S., T. Heiskanen, and W.H. Ray, Solid Catalyzed Olefin Polymerization. Chemical Engineering Progress, 1988. 84(11): p. 56-62.

53. Soares, J.B.P. and A.E. Hamielec, Deconvolution of Chain-Length Distributions of Linear-Polymers Made by Multiple-Site-Type Catalysts. Polymer, 1995. 36(11): p. 2257-2263.

54. Vickroy, V.V., H. Schneider, and R.F. Abbott, The Separation of Sec Curves of Hdpe into Flory Distributions. Journal of Applied Polymer Science, 1993. 50(3): p. 551-554.

55. Lin, S.T. and S.I. Sandler, Infinite dilution activity coefficients from ab initio solvation calculations. Aiche Journal, 1999. 45(12): p. 2606-2618.

56. Lacombe, R.H. and I.C. Sanchez, Statistical Thermodynamics of Fluid Mixtures. Journal of Physical Chemistry, 1976. 80(23): p. 2568-2580.

57. Khare, N.P., et al., Steady-state and dynamic modeling of commercial slurry high-density polyethylene (HDPE) processes. Industrial \& Engineering Chemistry Research, 2002. 41(23): p. 5601-5618.

58. Redlich, O. and J.N.S. Kwong, On the Thermodynamics of Solutions .5. An Equation of State - Fugacities of Gaseous Solutions. Chemical Reviews, 1949. 44(1): p. 233-244.

59. Soave, G., Equilibrium Constants from a Modified Redlich-Kwong Equation of State. Chemical Engineering Science, 1972. 27(6): p. 1197-\&.

60. Lichtenthaler RN, D.A.E., Prausnitz JM., Molecular Thermodynamics of Fluid Phase Equilibria. 3rd ed. ed. 1998, New York: Prentice Hall, Inc. 714-717.

61. Nasri, Z. and H. Binous, Applications of the Soave-Redlich-Kwong equation of state using Mathematica (R). Journal of Chemical Engineering of Japan, 2007. 40(6): p. 534-538.

62. Keii, T., Kinetics of Ziegler-Natta Polymerization. 1972, Kodansa LTD: Tokyo.

63. Kissin, Y.V., In Isospecific Polymerization of Olefins with Heterogeneous Ziegler-Natta Catalysts. 1985, New York: Springer-Verlag.

64. Kosek, J., et al., Dynamics of particle growth and overheating in gas-phase polymerization reactors. Chemical Engineering Science, 2001. 56(13): p. 3951-3977.

65. Hutchinson, R.A. and W.H. Ray, Polymerization of Olefins through Heterogeneous Catalysis - the Effect of Condensation Cooling on Particle Ignition. Journal of Applied Polymer Science, 1991. 43(7): p. 1387-1390.

66. Natta, G. and I. Pasquon, The Kinetics of the Stereospecific Polymerization of Alpha-Olefins. Advances in Catalysis, 1959. 11: p. 1-66.

67. Hakim, S., M. Nekoomanesh, and M.A. Nieat, Investigating the behaviour of a Bi-supported SiO2/TiCl4/THF/MgCl2 catalyst in slurry ethylene polymerization: Activity and molecular weight. Iranian Polymer Journal, 2008. 17(3): p. 209-216. 
68. Merquior, D.M., E.L. Lima, and J.C. Pinto, Modeling of particle fragmentation in heterogeneous olefin polymerization reactions, 2 - A two phase model. Macromolecular Materials and Engineering, 2005. 290(6): p. 511-524.

69. Bohm, L.L., The ethylene polymerization with Ziegler catalysts: Fifty years after the discovery. Angewandte Chemie-International Edition, 2003. 42(41): p. 5010-5030.

70. Smith, P., H.D. Chanzy, and B.P. Rotzinger, Drawing of Virgin Ultrahigh Molecular-Weight Polyethylene - an Alternative Route to High-Strength High Modulus Materials .2. Influence of Polymerization Temperature. Journal of Materials Science, 1987. 22(2): p. 523-531.

71. Di Martino, A., Morphogenesis of $\mathrm{MgCl}_{2}$-Supported Catalysts. 2006. p. 299.

72. Kiparissides, C., et al., Experimental and theoretical investigation of solubility and diffusion of ethylene in semicrystalline $P E$ at elevated pressures and temperatures. Journal of Applied Polymer Science, 2003. 87(6): p. 953-966.

73. Lemstra, P.J., et al., Chain mobility in polymer systems between solid and melt: Lamellar doubling. Abstracts of Papers of the American Chemical Society, 1999. 218: p. U636-U637.

74. Kurelec, L., S. Rastogi, and P.J. Lemstra, Chain mobility in polymer systems between solid and melt: Sintering via the mobile hexagonal phase. Abstracts of Papers of the American Chemical Society, 1999. 218: p. U644-U645.

75. Choi, K.Y. and W.H. Ray, Polymerization of Olefins through Heterogeneous Catalysis .2. Kinetics of Gas-Phase Propylene Polymerization with ZieglerNatta Catalysts. Journal of Applied Polymer Science, 1985. 30(3): p. 10651081.

76. Kissin, Y.V., R.I. Mink, and T.E. Nowlin, Ethylene polymerization reactions with Ziegler-Natta catalysts. I. Ethylene polymerization kinetics and kinetic mechanism. Journal of Polymer Science Part a-Polymer Chemistry, 1999. 37(23): p. 4255-4272.

77. Karol, F.J., S.C. Kao, and K.J. Cann, Comonomer Effects with High-Activity Titanium-Based and Vanadium-Based Catalysts for Ethylene Polymerization. Journal of Polymer Science Part a-Polymer Chemistry, 1993. 31(10): p. 25412553.

78. Chien, J.C.W., et al., Polymerizations of olefins and diolefins catalyzed by monocyclopentadienyltitanium complexes containing a (dimethylamino)ethyl substituent and comparison with ansa-zirconocene systems. Journal of Polymer Science Part a-Polymer Chemistry, 1998. 36(2): p. 319-328.

79. Pasquet, V. and R. Spitz, Irreversible Activation Effects in Ethylene Polymerization. Makromolekulare Chemie-Macromolecular Chemistry and Physics, 1993. 194(2): p. 451-461.

80. Lynch, D.T. and S.E. Wanke, Reactor Design and Operation for Gas-Phase Ethylene Polymerization Using Ziegler-Natta Catalysts. Canadian Journal of Chemical Engineering, 1991. 69(1): p. 332-339.

81. Xu, Z.G., S. Chakravarti, and W.H. Ray, Kinetic study of olefin polymerization with a supported metallocene catalyst. I. Ethylene/propylene copolymerization in gas phase. Journal of Applied Polymer Science, 2001. 80(1): p. 81-114.

82. Pimplapure, M.S. and G. Weickert, Catalytic polymerization of liquid propylene: Effect of low-yield hexene prepolymerization on kinetics and morphology. Macromolecular Rapid Communications, 2005. 26(16): p. 12941298. 
83. Shaffer, W.K.A. and W.H. Ray, Polymerization of olefins through heterogeneous catalysis .18. A kinetic explanation for unusual effects. Journal of Applied Polymer Science, 1997. 65(6): p. 1053-1080.

84. Bohm, L.L., On the Copolymerization of Ethylene and Alpha-Olefins with Ziegler-Catalysts. Makromolekulare Chemie-Macromolecular Chemistry and Physics, 1981. 182(11): p. 3291-3310.

85. Hindryckx, F., et al., Ethylene polymerisation by a high activity $\mathrm{MgCl} 2$ supported Ti catalyst in the presence of hydrogen and/or 1-octene. Polymer, 1998. 39(3): p. 621-629.

86. Yamamoto, A., Organotransition Metal Chemistry. 1986, New York: WileyInterscience.

87. Brookhart, M. and D.M. Lincoln, Comparison of Migratory Aptitudes of Hydride and Alkyl-Groups in Beta-Migratory Insertion Reactions of $C p](P(O m e) 3) R h(C 2 h 4) R+(R=H$, Ch2ch3). Journal of the American Chemical Society, 1988. 110(26): p. 8719-8720.

88. Boucheron, B., Mass-Transfer Phenomenon near Coordinated AnionicPolymerization in 1-Butene Solution in Relation to Hydrogen Effect. European Polymer Journal, 1975. 11(2): p. 131-138.

89. Pijpers, E.M.J. and B.C. Roest, Effect of Hydrogen on Ziegler-Natta Polymerization of 4-Methyl-1-Pentene. European Polymer Journal, 1972. 8(10): p. 1151-\&.

90. Mikenas, T.B., et al., Ethylene polymerization with supported vanadiummagnesium catalyst: Hydrogen effect. Macromolecular Chemistry and Physics, 2001. 202(4): p. 475-481.

91. McKenna, T.F. and J.B.P. Soares, Single particle modelling for olefin polymerization on supported catalysts: A review and proposals for future developments. Chemical Engineering Science, 2001. 56(13): p. 3931-3949.

92. Echevskaya, L.G., et al., Molecular mass characteristics of polyethylene produced with supported vanadium-magnesium catalysts. Polymer International, 2006. 55(2): p. 165-170.

93. Fernandes, F.A.N. and L.M.F. Lona, Multizone circulating reactor modeling for gas-phase polymerization. I. Reactor modeling. Journal of Applied Polymer Science, 2004. 93(3): p. 1042-1052.

94. Shan, C.L., J.B.P. Soares, and A. Penlidis, HDPE/LLDPE reactor blends with bimodal microstructures - part 1: mechanical properties. Polymer, 2002. 43(26): p. 7345-7365.

95. Chu, K.J., et al., Effect of prepolymerization and hydrogen pressure on the microstructure of ethylene/1-hexene copolymers made with $\mathrm{MgCl2}$-supported TiCl3 catalysts. European Polymer Journal, 2000. 36(1): p. 3-11.

96. Czaja, K. and B. Krol, Two-step polymerization of propylene over MgCl2supported titanium catalyst. Macromolecular Chemistry and Physics, 1998. 199(3): p. 451-455.

97. $\mathrm{Wu}$, L., D.T. Lynch, and S.E. Wanke, Kinetics of gas-phase ethylene polymerization with morphology-controlled MgCl2-supported TiCl4 catalyst. Macromolecules, 1999. 32(24): p. 7990-7998.

98. Pater, J.T.M., G. Weickert, and W.P.M. van Swaaij, Propene bulk polymerization kinetics: Role of prepolymerization and hydrogen. Aiche Journal, 2003. 49(1): p. 180-193. 
99. Shan, C.L.P., J.B.P. Soares, and A. Penlidis, HDPE/LLDPE reactor blends with bimodal microstructures - Part II: rheological properties. Polymer, 2003. 44(1): p. 177-185.

100. Abedi, S. and N. Hassanpour, Preparation of bimodal polypropylene in twostep polymerization. Journal of Applied Polymer Science, 2006. 101(3): p. 1456-1462.

101. Ahn, T.O., et al., Modification of a Ziegler-Natta catalyst with a metallocene catalyst and its olefin polymerization behavior. Polymer Engineering and Science, 1999. 39(7): p. 1257-1264.

102. Tsubaki; Kazumi; Morinaga; Hiroshi; Matsuo; Yoshiho; Iwabuchi; Takeshi; "Two step process for polymerizing ethylene" U.S.Patent 4357448, November 2, 1982.

103. Mirzaei, A., M. Vakili, and N. Tafi, Prepolymerization of ethylene with a Ziegler-Natta catalyst. Journal of Applied Polymer Science, 2007. 105(5): p. 2703-2711.

104. Pater, J.T.M., G. Weickert, and W.P.M. van Swaaij, Polymerization of liquid propylene with a 4th generation Ziegler-Natta catalyst - influence of temperature, hydrogen and monomer concentration and prepolymerization method on polymerization kinetics. Chemical Engineering Science, 2002. 57(16): p. 3461-3477.

105. Pater, J.T.M., et al., High precision prepolymerization of propylene at extremely low reaction rates-kinetics and morphology. Chemical Engineering Science, 2001. 56(13): p. 4107-4120. 


\section{Acknowledgements}

Highest thanks to the munificent and the merciful God for granting me this opportunity to research in the area that I have been working in for more than 12 years.

Next, I would like to convey my special thanks to my supervisor, Prof. Gunter Weickert, for giving me the opportunity to come to the University of Twente to undertake my $\mathrm{PhD}$ project on the topic of "Comparison of Catalytic Ethylene Polymerization in Slurry and Gas Phase". I found him incessantly active and keen to share his knowledge and experiences with all his PhD students. His scientific guidance, trustworthy advice, patience and fortitude in the face of all my mistakes - which all come from his long experience in academia and industrial research in the polyolefin area - increasingly broadened and improved both my knowledge and understanding of polyolefins. Many thanks also to his wife Martina for her unfailing kindness and hospitality.

I would like to express my deep gratitude to Prof. Ludwig Bohm, Prof. W.P.M. van Swaaij, Prof. Vahid Haddadi Asl, Dr. Martin van sint Annaland and Dr. Gerben Meier who proofread the draft of my thesis. This work was financially supported by the DPI (Dutch Polymer Institute). I would like to thank all those from academia and industry who kindly shared their knowledge and experience with me during these years. Special thanks are due to Prof. Stamhuis and Prof. Vincenzo Busico for making all the necessary arrangements. Many thanks to Dr. Jochem Pater and Dr. Gerben Meier from the Lyondellbasell Company for all the support, scientific discussion and important information that they contributed.

I am also grateful to NPC and to the NPC-RT Company for all help given both before and during my $\mathrm{PhD}$ research. I wish to express my deepest gratitude to Prof. Taeb, Mr. Nematzadeh, Prof, Haddadi Asl, Prof, Nekomanesh, Mr. Afshin, Mr. Harraf, Dr. Ghafelebashi, Dr. Mellati, Mrs. Rasouli, Mrs. Hashemi for all their support and practical help.

I wish also thank to my colleagues and their families from the former IPP (Industrial Polymerization Processes) Group: Dr. Yahya Banat, Dr. Makarand Pimplarpure, Dr. Ravindra Tube, Dr. Mohammad Al-haj Ali, Dr. Eric Ericson, Shankara Narayanam and Ide Enggelsma. Special thanks to Dr. Yahya Banat for all scientific discussion and guidance. I also wish to thank the secretaries of the IPP, especially Annet Rip and Bartie Bruggink-de Braal, for their kind help in many ways; especially in translating the many letters in Dutch and in helping me to complete more than one hundred forms.

The experimental work would not have been completed without the help of many people. I would therefore like to acknowledge and thank the staff of the Polymerization Reaction Technology group: Ide Enggelsma, Eric-Jan Prinsen, Norbert Dülker; HDL's staff (Hoge Druk Lab): Gert Banis, Fred ter Borg, Geert Monnik, Karst van Bree, John Agterhorst; Andries Jekel for HT-GPC measurements (Rijksuniversiteit Groningen), Mrs. Zare for DSC measurements (NPC-RT), Ali Safinejad for Deconvolution and Aspen Polymer Plus software, Mr. V. Seydewitz for SEM analysis (Halle University), Mrs. S. Goerlitz for TEM analysis (Halle University).

Thanks to Mr. Paul Bakker for his fast and precise correction of this thesis. 
Many thanks also to Iranian community in Netherlands, particularly Dr. Masoud Sharif, Dr. M. Ali Sharifi, Dr. Abbas Farshad, Dr. Ali Abkar, Dr. Bahman Farhadi Bansouleh, Dr. Saeid Talebi, Dr. Masoud Kheirkhah, Mr. Farhang Sargordi, Mr. Nima Moein, Mr. Saeed Sedghi, Mr. Kasra Garakoui, Mr. Mohammad Abou Ali and their families. They provide me and my family with such a pleasant environment that it felt somewhat as if I were living in my lovely country of Iran.

More importantly, a great thanks to my parents, parents-in-law, dear brothers (Hamid, Hamed and Abbas), my brothers- and sister-in-law and their families for their blessing help and support.

Most importantly, heartfelt thanks to my precious wife Atefeh who allowed me to follow my dream and constantly encouraged me forward in my research. Many thanks to my lovely sons Matin and Mobin for their love, trust and invariable respect for their parents. You all left your relatives and friends and our lovely country of Iran, and came here to support me. At first, it was very difficult, but soon you all learned the Dutch and English languages and adapted yourselves to the Netherlands. You are amazing and I am incredibly proud of you all.

Thank you so much to many people whom I missed to mentioned their name individually for helping me to create this work during these years. 


\section{Curriculum Vitae}

Majid Daftaribesheli was born on $16^{\text {th }}$ of August 1966 in Ghaemshahr, Mazandran, Iran. After completing high school in physics and mathematics in 1984, he was accepted at the Abadan Institute of Technology, Iran. After being awarded his Bachelor's degree in Petrochemical Engineering in 1989, he went to work at the LLDPE plant of ARPC (Arak Petrochemical Company) in 1990. In 1994, he was accepted at Tehran University to study for his Master's degree in Chemical Engineering, and he graduated in 1997.

His experiences and knowledge of polyolefins grew at ARPC. In 1999, he was appointed as a head manager of the LLDPE\&Bu-1 plant in ARPC. During his career at ARPC, he was also appointed as a part-time lecturer at the chemical engineering department of Azad University in Arak, Iran.

In 2001, he joined the Research and Development department of the NPC (National Petrochemical Company of Iran). In 2002, he was appointed as a managing director of the Pouyesh Company, an affiliate of NPC, with responsibility for industrial research and pilot plant facilities.

In 2003, after Pouyesh merged with NPC's R\&D department and the establishment of the R\&T Company, he was appointed as a member of the board of R\&T and the director of the Pouyesh Centre.

In September 2004, he was accepted to study for his $\mathrm{PhD}$ on the topic of this thesis under the supervision of Prof. Dr.-Ing. Habil Gunter Weickert in the Industrial Polymerization Processes (IPP) group at the University of Twente, Enschede, the Netherlands. 